\title{
Problemas de corte com sobras aproveitáveis e eliminação de simetrias
}

\author{
Ricardo Luiz de Andrade Abrantes
}

\author{
TEXTO APRESENTADO \\ AO \\ Instituto DE MATEMÁTICA E EstATÍSTICA \\ DA \\ UNIVERSIDADE DE SÃO PAULO \\ PARA \\ OBTENÇÃO DE TÍULO \\ DE \\ DOUTORADO EM CIÊNCIAS
}

\author{
Programa: Ciência da Computação \\ Orientador: Prof. Dr. Ernesto G. Birgin
}

Durante o desenvolvimento deste trabalho o autor recebeu auxílio financeiro da CAPES

São Paulo, dezembro, 2012 



\section{Problemas de corte com sobras aproveitáveis e eliminação de simetrias}

Esta versão da dissertação/tese contém as correções e alterações sugeridas pela Comissão Julgadora durante a defesa da versão original do trabalho, realizada em 20/09/2012. Uma cópia da versão original está disponível no Instituto de Matemática e Estatística da Universidade de São Paulo.

Comissão julgadora:

- Prof. Dr. Ernesto Julian Goldber Birgin (orientador) - IME-USP

- Profa. Dra. Yoshiko Wakabayashi - IME-USP

- Profa. Dra. Kelly Cristina Poldi - UNIFESP

- Prof. Dr. Horacio Hideki Yanasse - INPE

- Prof. Dr. Reinaldo Morabito Neto - UFSCar 



\section{Agradecimentos}

Gostaria de agradecer à minha mãe, que sempre me deu todo apoio para trilhar este caminho.

Agradeço ao Ernesto, por estar sempre presente, pelas conversas que tivemos ao longo de todos esses anos e por todas as contribuições ao trabalho.

Agradeço também à Débora Ronconi e ao Reinaldo Morabito pelas sugestões e a todos os membros da banca julgadora por todas as recomendações de melhorias do texto, que também foram muito importantes.

Agradeço aos meus amigos que sempre estiveram presentes. Sem eles, o caminho teria sido muito mais difícil. Foram muitos que ajudaram de alguma forma a realização deste trabalho. Amigos da salinha, do IME e de fora da USP também. Em particular, tenho profunda gratidão aos seguintes: Marcelo Hashimoto, Luís Henrique Bustamante de Morais, Livy Maria Real Coelho, Ellen Hidemi Fukuda, Celina Maki Takemura, Alexandre César Tavares Vidal, Daniel de Angelis Cordeiro, Francisco Calmon Sobral, Rafael Durbano Lobato, Gordana Manić, Márcio Oshiro, Paulo Salem e Carlos Cardonha. 

The greatest challenge to any thinker is stating the problem in a way that will allow a solution.

- Bertrand Russel 


\section{Resumo}

Andrade, R. Problemas de corte com sobras aproveitáveis e eliminação de simetrias. 2012. 113 p. Tese (Doutorado) - Instituto de Matemática e Estatística, Universidade de São Paulo, São Paulo, 2012.

No presente trabalho estudamos duas variações do problema de empacotamento de itens retangulares idênticos, permitindo rotações de 90 graus, em um poliedro. Um consiste em encontrar a maior quantidade de itens retangulares idênticos que podem ser empacotados em um poliedro. $\mathrm{O}$ outro consiste em encontrar o poliedro de um determinado tipo com menor área para empacotar uma quantidade fixa de itens retangulares idênticos. Desenvolvemos restrições de eliminação de simetrias para este problema, o que tornou a resolução do mesmo mais eficiente, por métodos do tipo branch-\&-bound.

Estudamos também o problema de corte no qual há uma determinada demanda (de itens) a ser cortada e um conjunto de objetos disponíveis. Desejamos satisfazer a demanda minimizando o custo dos objetos utilizados e, dentre as diferentes possibilidades de se fazer isso, desejamos aquela que maximize as sobras aproveitáveis. De forma geral, sobras aproveitáveis podem ser entendidas como regiões retangulares de um objeto que possuem altura e largura iguais ou superiores a de um item de referência e representam sobras do processo de corte que podem se tornar objetos e serem reaproveitadas em um novo procedimento de corte. Apresentamos modelos de otimização em dois níveis para duas variações do problema de corte com sobras aproveitáveis a saber: o problema de corte de itens retangulares em dois estágios e o problema de corte de itens retangulares não guilhotinado. Como formas de resolver os modelos propostos, apresentamos reformulações destes modelos de programação em dois níveis em modelos de programação inteira mista. Lidamos também com uma variação do problema de corte com sobras aproveitáveis considerando a minimização da quantidade de sobras. Aplicamos restrições de eliminação de simetrias aos modelos desenvolvidos para o problema de corte de itens retangulares com sobras aproveitáveis, a fim de resolver instâncias maiores, e desenvolvemos uma estratégia de solução alternativa para os modelos. Os modelos desenvolvidos foram implementados computacionalmente e fomos capazes de resolver instâncias pequenas dos problemas em questão.

Palavras-chave: Problemas de empacotamento, problemas de corte de estoque, sobras aproveitáveis, eliminação de simetrias, otimização. 


\section{Abstract}

Andrade, R. Cutting stock problems with usable leftover and symmetry breaking. 2012. 113 p. Tese (Doutorado) - Instituto de Matemática e Estatística, Universidade de São Paulo, São Paulo, 2012.

In this work we study two variations of the packing problem where identical rectangular items must be packed into a polyhedron. One of the variations consists in finding the largest amount of rectangular items that can fit in a polyhedron. The other one consists in finding a minimal area polyhedron of a certain type that packs a set of rectangular identical items. We present some symmetry-breaking constraints that reduce the computational effort in solving those problems through a branch-\&-bound method.

We also studied the cutting stock problem where there are some items to be cut from a set of rectangular objects and we need to satisfy the demand of items to be cut minimizing the cost of the used objects and, among the different ways of doing this, we want that which maximize the usable leftovers. Loosely speaking,usable leftovers can be understood as rectangular regions in an object that has the width and the height greater than or equal to the ones of a reference item. These leftovers can be seen as leftovers from a cutting process that will become items in a new cutting process. We present bilevel programming models to two variations of this problem with usable leftovers: the two-stage cutting stock problem of rectangular items and the non-guillotine cutting stock problem of rectangular items. In order to solve the proposed models we present also MIP reformulations of these bilevel programming problem models. We also developed some symmetry breaking constraints in order to accelerate the solving process of those models. The developed models were computationally programmed and we were able to solve small instances of the proposed problems.

Keywords: cutting problems, packing problems, usable leftover, symmetry breaking constraints, optimization. 


\section{Sumário}

1 Introdução $\quad 1$

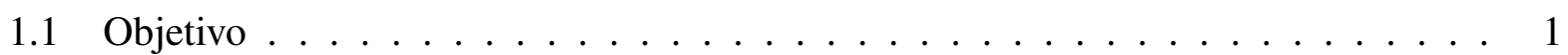

1.2 Revisão bibliográfica . . . . . . . . . . . . . . . . . . . 2

1.3 Organização do trabalho . . . . . . . . . . . . . . . . . . 6

2 Simetrias no empacotamento de retângulos em um poliedro $\quad 7$

2.1 Modelos de programação inteira mista . . . . . . . . . . . . . . . . . 8

2.2 Restrições de eliminação de simetrias . . . . . . . . . . . . . . . . . . . . 11

2.3 Experimentos numéricos . . . . . . . . . . . . . . . . . . 13

2.3.1 Minimizando a dimensão do objeto . . . . . . . . . . . . . . . 15

2.3.2 Empacotando a maior quantidade de retângulos possível . . . . . . . . . . 17

2.4 Conclusões . . . . . . . . . . . . . . . . . . . . . . . 21

3 Problema de corte em dois estágios com sobras aproveitáveis 25

3.1 Um modelo para o problema de corte em dois estágios . . . . . . . . . . . . . 26

3.2 Considerando as sobras aproveitáveis . . . . . . . . . . . . . . 27

3.3 Outro modelo para o problema de corte dois estágios . . . . . . . . . . . . . 34

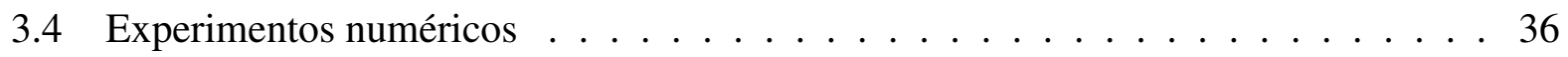

4 Problemas de corte de itens retangulares com sobras aproveitáveis $\quad 57$

4.1 Um modelo para o problema de corte de itens retangulares . . . . . . . . . . . . 57

4.2 Considerando sobras aproveitáveis guilhotinadas . . . . . . . . . . . . 60

4.3 Considerando sobras aproveitáveis genéricas . . . . . . . . . . . . . . 71

4.4 Experimentos Numéricos . . . . . . . . . . . . . . . . . . . . . . . . . . 79

5 Melhorias nos modelos para o problema de corte com sobras 89

5.1 Restrições de eliminação de soluções equivalentes e simétricas . . . . . . . . . . . 90

5.1.1 Eliminação de soluções equivalentes nos modelos $\mathcal{M}_{0}^{G}$ e $\mathcal{M}_{1}^{G} \ldots \ldots$. . . . 90

5.1.2 Eliminação de soluções equivalentes nos Modelos $\mathcal{M}_{2}^{G}$ e $\mathcal{M}_{3}^{G} \ldots$. . . . . 91

5.1.3 Restrições de eliminação de simetrias do tipo "acima e à direita" para itens do mesmo tipo . . . . . . . . . . . . . . . . . . . . . 92 
5.1.4 Restrições de eliminação de simetrias específicas para os Modelos $\mathcal{M}_{2}^{G}$ e

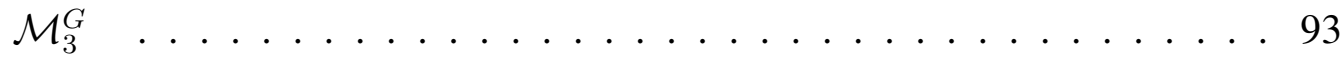

5.1.5 Experimentos . . . . . . . . . . . . . . . . . 94

5.2 Estratégia de duas fases . . . . . . . . . . . . . . . . . . . 103

5.2.1 Experimentos . . . . . . . . . . . . . . . 103

5.3 Minimizando a quantidade de sobras aproveitáveis . . . . . . . . . . 106

5.3 .1 Experimentos . . . . . . . . . . . . . . . 108

6 Conclusões 113

$\begin{array}{ll}\text { Referências Bibliográficas } & 115\end{array}$ 


\section{Capítulo 1}

\section{Introdução}

Problemas de empacotamento consistem em alocar itens em um ou mais objetos de forma que os itens não se sobreponham. Estudamos duas variações do problema de empacotamento em que temos apenas um objeto e itens retangulares idênticos, aos quais permitimos rotações ortogonais. Modelamos o problema de empacotamento como um problema de programação inteira mista (MIP - Mixed Integer Programming) e estudamos diferentes restrições de eliminação de simetrias. Consideramos como soluções simétricas, soluções que possuem o mesmo valor de função objetivo e que diferem umas das outras pela troca de posição de itens idênticos. Restrições de eliminação de simetria são restrições adicionais impostas a um modelo, com o objetivo de eliminar soluções simétricas. Como alguns métodos de solução baseiam-se na enumeração de soluções, a eliminação de soluções simétricas em um modelo pode acelerar o processo de resolução do mesmo.

Problemas de corte de estoque ou, simplesmente, problemas de corte consistem em obter um conjunto de padrões de corte para produzir um conjunto de itens a partir de um conjunto de objetos, minimizando ou maximizando uma determinada função objetivo. Problemas de corte aparecem em diferentes contextos e possuem uma grande quantidade de aplicações na indústria [Wäscher07], por exemplo, o corte de peças minimizando desperdício de material na indústria moveleira [Morabito98, Morabito00]. No presente trabalho estudamos, também, um problema de corte de estoque no qual há uma determinada demanda de itens retangulares a ser cortada e um conjunto de objetos retangulares disponíveis. Desejamos satisfazer a demanda minimizando o custo dos objetos utilizados e, dentre as diferentes possibilidades de se fazer isso, desejamos aquela que maximize o valor das sobras aproveitáveis. Denominamos este tipo de problema de problema de corte bidimensional com sobras aproveitáveis ou, simplesmente, problema de corte com sobras aproveitáveis [Cherri09a]. Em um padrão de corte podem ser identificados itens e sobras. As sobras podem ser classificadas em sobras aproveitáveis e descartes. De forma geral, sobras aproveitáveis podem ser entendidas como regiões retangulares de um objeto que possuem altura e largura iguais ou superiores a dimensões de referência e que representam sobras de um processo de corte. Tais sobras retangulares são consideradas aproveitáveis pois podem ser utilizadas como objetos em um novo procedimento de corte. Consideramos que descarte é toda sobra que não é aproveitável.

\subsection{Objetivo}

Problemas de corte e empacotamento podem ser modelados como problemas de otimização com variáveis inteiras. Para resolver esses problemas são utilizados métodos do tipo branch-andbound, apresentados inicialmente em [Land60], que enumeram as possíveis soluções do problema. Assim, é interessante obter modelos que contemplem um espaço de soluções pequeno, permitindo que métodos do tipo branch-and-bound possam enumerar os candidatos a solução de um problema 
mais rapidamente e identificar a solução desejada de forma mais eficiente. Inicialmente consideramos dois problemas de empacotar itens retangulares idênticos em um poliedro. O primeiro consiste em identificar qual é o poliedro de menor área que pode empacotar um conjunto dado de retângulos idênticos. O segundo consiste em, dado um poliedro, identificar a quantidade máxima de retângulos que podem ser empacotados nele. Baseados em modelos de programação inteira mista para esses problemas, temos o objetivo de desenvolver restrições de eliminação de simetrias que melhorem o desempenho de solução dos modelos por métodos do tipo branch-and-bound.

Estudamos também problemas de corte com sobras aproveitáveis. Como dissemos anteriormente, consideramos que sobras aproveitáveis são regiões retangulares tais que suas dimensões são iguais ou superiores a dimensões de referência. Mais especificamente, uma sobra aproveitável é uma região retangular em um objeto que satisfaz pelo menos uma das seguintes afirmações:

- Suas dimensões são iguais ou superiores a dimensões mínimas dadas.

- Suas dimensões são suficientemente grandes para cortar pelo menos um item de um catálogo de itens dado (que pode ser a lista de itens demandados).

Claramente a primeira afirmação é um caso particular do segunda (podemos ter uma lista com um único item com as dimensões mínimas desejadas). A principal motivação para lidar com problemas de corte com sobras é a possibilidade de reaproveitamento de material, que tem importância em processos industriais, tanto do ponto de vista econômico como ambiental.

Estamos interessados no problema de corte bidimensional com sobras aproveitáveis, no qual deseja-se cortar um conjunto finito de itens retangulares a partir de um conjunto finito de objetos retangulares, sem qualquer restrição no tipo do padrão de corte. Porém, optamos por considerar, inicialmente, uma variação mais simples deste problema no qual temos a restrição adicional de que o padrão de corte deva ser do tipo dois estágios. Padrões de corte do tipo dois estágios são padrões nos quais é feita uma sequência de cortes paralelos a um dos lados do objeto, caracterizando o primeiro estágio, e, em seguida, uma outra sequência de cortes ortogonais aos primeiros, obtendo-se os itens desejados. Em padrões do tipo dois estágios é permitido um terceiro corte, conhecido por trimming, para separar alguns itens de eventuais sobras [Gilmore65, Beasley85a, Lodi03, Morabito96].

Assim, nosso objetivo é propor modelos que capturem adequadamente a natureza dos problemas de corte com sobras aproveitáveis, considerando inicialmente o caso particular no qual o padrão de corte é restrito ao tipo dois estágios e depois considerando o caso não guilhotinado, bem como, propor estratégias para resolvê-los. Já que, nos problemas com sobras aproveitáveis, desejamos uma solução que, dentre as soluções que minimizam o custo dos objetos, maximize o valor das sobras aproveitáveis, consideramos natural modelá-los como problemas de otimização em dois níveis ou programação em dois níveis [Bard98, Dempe02]. Desejamos estudar possibilidades de resolver esses modelos através de reformulações em problemas de programação inteira mista e desenvolver restrições para a eliminação de soluções simétricas.

\subsection{Revisão bibliográfica}

É possível encontrar na literatura centenas de trabalhos sobre diversos problemas de corte e empacotamento, conforme indicam as revisões bibliográficas [Lodi02, Dyckhoff97, Bischoff95, Dowsland92, Finke92, Sweeney92], diversas edições especias sobre o assunto em revistas como European Journal of Operational Research, Information Systems and Operational Research, International Transactions in Operational Research e a página web do EURO Special Interest Group on Cutting and Packing em http://paginas.fe.up.pt/ esicup/. A seguir faremos uma revisão bibliográfica dos trabalhos que acreditamos estarem mais relacionados com esta tese. 
Considerando a grande quantidade de trabalhos na área e a diversidade de nomes utilizados para um mesmo problema, Dyckhoff elaborou, em [Dyckhoff90], uma tipologia para os problemas de corte e empacotamento com o intuito de classificar os problemas de acordo com sua estrutura lógica e padronizar a nomenclatura. Sua tipologia é baseada em quatro características principais: dimensionalidade, tipo de atribuição, tipo dos objetos e tipo dos itens. Mais recentemente, em [Wäscher07], a tipologia apresentada em [Dyckhoff90] foi estendida levando-se em consideração as dimensões e formas de objetos e itens, bem como, foram definidas categorias para os tipos básicos de problemas de corte e empacotamento. Trabalhamos inicialmente com duas variações do problema de empacotamento de retângulos idênticos em poliedros. Este problema pode ser classificado como 2/B/O/C, de acordo com [Dyckhoff90]. De acordo com a tipologia de [Wäscher07], se encaixa na categoria bidimensional, retangular, Identical Item Packing Problem( ou IIPP). Tal problema e suas variações aparecem em diversos contextos, como no problema de carregamento de palete do produtor (pallet loading problem) ou o problema de empacotar contêineres em um navio.

Em [Stoyan98] são apresentados modelos para o problema de empacotar retângulos em retângulos e para o problema de empacotar círculos em retângulos. É proposto um método para solução do problema baseado em uma combinação do método de branch-and-bound e do gradiente reduzido. Em [Morabito98] é apresentada uma heurística simples e eficiente para resolver o problema de empacotar retângulos idênticos em retângulos. Uma revisão extensa sobre o problema de empacotar retângulos em retângulos é apresentada em [Lodi02]. Em [Lins03] é apresentado um procedimento recursivo para empacotar retângulos em retângulos e peças no formato de $L$ (ou simplesmente peças $L$ ). Em [Birgin05b] é sugerida uma outra estrutura de dados para melhorar o desempenho computacional do procedimento de empacotar retângulos em peças $L$. Em [Birgin10a] é apresentado um método recursivo, baseado na combinação de um método heurístico e em uma abordagem para empacotar retângulos em peças $L$, para resolver o problema de empacotar retângulos idênticos, com a possibilidade de rotações de 90 graus, em um retângulo maior.

Em [Birgin05a] é apresentado o método das sentinelas para o problema de empacotar itens quaisquer em regiões convexas do espaço. O método baseia-se na definição de um conjunto de pontos, chamados sentinelas, e na resolução de um problema de otimização contínua. Em [Birgin10b] o conceito de sentinelas é estendido para identificar se dois polígonos arbitrários possuem intersecção ou não. É apresentada uma aplicação na qual as sentinelas são utilizadas para modelar restrições de não-sobreposição em um problema de empacotar polígonos em conjuntos convexos. Em [Birgin06] é estudado o problema de empacotar a maior quantidade de retângulos idênticos, com a possibilidade de rotações de 90 graus, em regiões convexas. É apresentado um método baseado em um modelo de programação não linear para encontrar a maior quantidade de retângulos que podem ser empacotados em uma dada região. $\mathrm{O}$ método considera uma sequência de problemas de empacotar uma quantidade fixa de retângulos em uma determinada região. Essa sequência de problemas é montada de forma que a quantidade de retângulos a serem alocados é crescente. Quando um determinado problema com $k$ retângulos não pode ser resolvido dentro do tempo estipulado, conclui-se que a quantidade máxima de retângulos que podem ser acomodados na região em questão é $k-1$. Em [Birgin09] é considerado um problema similar ao considerado em [Birgin06], com a diferença de que o objeto no qual os itens devem ser empacotados pode sofrer uma rotação arbitrária. Em [Korf08] é tratado o problema de identificar um retângulo de área mínima que contém um conjunto de retângulos menores. São apresentadas duas formulações matemáticas desse problema como um problema de satisfação de restrições (Constraint Satisfaction Problem), que consideram apenas retângulos de dimensões inteiras. Em [Cassioli11] é apresentado um método heurístico para empacotar retângulos idênticos em uma região convexa.

Neste trabalho lidaremos também com problemas de corte com duas dimensões, nos quais 
desejamos uma seleção adequada de objetos aos quais todos os itens possam ser atribuídos. Temos diversos objetos de tipos diferentes e podemos ter muitos itens de poucos tipos distintos ou muitos itens de muitos tipos. Assim, os problemas de corte com os quais lidamos neste trabalho encaixam-se nas categorias 2/V/D/M ou 2/V/D/R, segundo [Dyckhoff90]. De acordo com a tipologia apresentada em [Wäscher07], os problemas de corte considerados neste trabalho encaixam-se na categoria bidimensional, retangular, Multiple Stock Size Cutting Stock Problem ou MSSCSP. Segundo [Wäscher07], problemas deste tipo são considerados uma extensão do problema clássico de corte de estoque bidimensional estudado em [Gilmore65], que consiste em um problema categorizado como bidimensional, retangular, Single Stock-Size Cutting Stock Problem ou SSSCSP. A seguir revisamos alguns trabalhos que lidam com problemas de corte de estoque do tipo bidimensional, retangular, SSSCSP e também MSSCSP.

Em [Gilmore65], é estudado o problema no qual um conjunto de itens retangulares de baixa heterogeneidade deve ser cortado a partir de um tipo de objeto e também algumas de suas variações. Dentre as variações consideradas, temos os problemas multiestágios, nos quais temos a restrição de que os itens devem ser obtidos a partir de cortes guilhotinados, realizados em dois ou mais estágios, de forma que em cada estágio é realizado um conjunto de cortes guilhotinados paralelos entre si. Posteriormente, em [Herz72], foi apresentado um método recursivo, baseado em uma discretização preliminar do problema de corte, que obtém soluções de forma mais eficiente do que através do método proposto em [Gilmore65]. Em [Christofides77] foi proposto um algoritmo baseado em uma estratégia de busca em profundidade. A ideia principal do algoritmo é gerar todos os possíveis padrões de corte, construindo uma árvore onde o processo de branching, em cada nível da árvore, está relacionado com cortes no objeto. Um limitante superior no valor máximo que pode ser obtido em cada nó é utilizado para realizar podas na árvore. Em [Beasley85b] é apresentado um método exato para resolver o mesmo tipo de problema, que consiste em usar uma relaxação lagrangiana, para uma formulação com variáveis binárias, como limitante para realizar podas em um procedimento baseado em árvore de busca. Para mais informações sobre o uso de relaxações lagrangianas, em problemas de programação inteira mista, ver [Fischer81]. Em [Beasley85a] são apresentados algoritmos heurísticos e um exato, baseado em programação dinâmica, para o mesmo problema de corte nas versões com e sem estágios. Em [Wang83] foram propostos dois métodos que geram padrões de corte através da junção vertical ou horizontal de retângulos obtidos a partir dos itens demandados ou combinações destes. Esta ideia para gerar padrões de corte foi explorada em [Viswanathan93] em combinação com uma estratégia do tipo best-first para a seleção dos melhores cortes. Em [Hifi97] são propostas melhoras ao algoritmo apresentado em [Viswanathan93] que consistem em obter melhores limitantes inferiores e superiores a partir do uso de heurísticas e da solução de instâncias do problema da mochila unidimensional relacionadas com o problema de corte original. Em [Cung00] são apresentadas extensões do método apresentado em [Hifi97] para variantes restritas do problema de corte de estoque bidimensional. Em [Cui11] são abordadas duas variações do problema de corte bidimensional guilhotinado de retângulos idênticos. Uma das variações não considera o comprimento da lâmina de corte, enquanto que a outra considera os casos em que a lâmina de corte é menor que o comprimento do objeto. Neste trabalho são considerados dois objetivos, o principal consiste em maximizar a quantidade de itens cortados e o secundário é minimizar a quantidade de cortes necessários para dividir os objetos em faixas.

Em [Morabito89, Morabito92] foi proposta uma abordagem baseada em grafos-E/OU para problemas de corte bidimensional irrestritos (problemas do tipo bidimensional, retangular, SSSCSP, segundo a tipologia apresentada em [Wäscher07]). O grafo-E/OU é utilizado para representar o espaço de soluções do problema e em seguida são utilizadas diferentes estratégias de busca para identificar a solução. Posteriormente, foram realizadas extensões deste trabalho para a resolução de outros tipos de problemas de corte [Morabito94, Arenales95, Morabito96, Morabito08]. Em particular, o trabalho [Morabito96] estende a abordagem por grafo-E/OU ao problema de corte 
bidimensional em multiestágios com diversos tipos de itens e objetos (bidimensional, retangular, MSSCSP, segundo a tipologia apresentada em [Wäscher07]). Em [Riehme96] é proposta uma abordagem para o problema de corte bidimensional em dois estágios com demandas extremas. Neste problema, há diversos tipos de itens e objetos e há itens com diferentes demandas. Esta proposta, baseada em [Gilmore65], consiste em primeiro resolver uma versão relaxada do problema, obtendo padrões de corte que preenchem a maior parte da demanda, e em seguida tratar os casos que sobram separadamente.

Considerando ainda trabalhos que lidam com problemas do tipo bidimensional, retangular, MSSCSP, podemos citar [Li96] no qual são apresentados modelos e heurísticas para resolver o problema de corte bidimensional em dois estágios com datas de vencimento e liberação, [Mornar97] no qual é desenvolvido um procedimento heurístico para um problema de corte de placas de circuitos impressos e [Babu99] que propõe heurísticas e algoritmos genéticos para encontrar padrões de corte que maximizem a área ocupada pelos itens, considerando diferentes tipos de objetos. Em [Alvarez-Valdes02] é abordado o problema de corte bidimensional, utilizando um esquema similar ao proposto em [Gilmore65]. Neste artigo é feita uma comparação computacional de diversos métodos heurísticos para resolver o subproblema do esquema proposto em [Gilmore65], ao invés de usar programação dinâmica. Em [Cui12] é tratado o problema de corte bidimensional em três estágios e o objetivo considerado é minimizar a soma dos custos dos objetos e dos cortes. É apresentado um procedimento baseado em geração de colunas para obter os padrões de corte com o objetivo de minimizar o custo dos cortes. Os custos de corte também são considerados explicitamente na função objetivo do modelo de programação linear apresentado. São realizados experimentos numéricos que ilustram que é possível obter soluções com menos cortes sem alterar o custo dos objetos.

Com relação a problemas de corte que consideram sobras aproveitáveis, embora não seja um conceito novo e, no contexto de problemas de corte de itens unidimensionais, existam diversos trabalhos considerando este conceito, como por exemplo [Roodman86, Scheithauer91, Sinuany-Stern94, Chu99, Gradisar05, Abuabara08, Cherri09b], encontramos apenas [Cherri09a] no contexto de problemas de corte bidimensional. O problema considerado nesse caso é um problema de corte de estoque do tipo bidimensional, retangular, MSSCSP. Neste trabalho é utilizada a abordagem do grafo-E/OU para a geração dos padrões de corte e são propostos alguns critérios que modificam o padrão gerado, tentando reduzir as perdas e criando sobras aproveitáveis. Para resolver o problema de corte com sobras, a autora propõe duas heurísticas, a GULOSA $A_{A}^{2 D}$ e a $\mathrm{RAG}_{A}^{2 D}$, que se baseiam nessa abordagem grafo-E/OU modificada. A heurística GuLOSA $A_{A}^{2 D}$ consiste em gerar um conjunto de padrões de corte, selecionar aquele que possuir a menor perda e menor quantidade de retalhos e usar este padrão a maior quantidade de vezes possível, de forma a não exceder a demanda ou quantidade de objetos daquele tipo disponíveis. Em seguida, atualizamse as demandas e o estoque e, caso a demanda não tenha sido satisfeita, repete-se o processo. A heurística $\mathrm{RAG}_{A}^{2 D}$ é baseada na heurística de arredondamento guloso [Poldi09]. A heurística de arredondamento guloso tem o objetivo de obter uma solução inteira aproximada para uma instância do modelo proposto em [Gilmore63], para o problema de corte unidimensional, a partir de uma solução relaxada (contínua). $\mathrm{A} \mathrm{RAG}_{A}^{2 D}$ consiste em aplicar a heurística de arredondamento guloso para obter uma solução inteira aproximada para o modelo proposto por Gilmore e Gomory em [Gilmore63], considerando padrões de corte bidimensionais (gerados pela abordagem do grafo-E/OU modificada para considerar sobras aproveitáveis). Em seguida é feita uma análise das perdas nos padrões da solução aproximada, comparando-as com um limitante superior précalculado. Se a sobra de algum padrão de corte não for aceitável, o padrão é rejeitado. A partir de todos os padrões rejeitados é montado um problema residual que é resolvido com a heurística $\operatorname{GulosA}_{A}^{2 D}$. 


\subsection{Organização do trabalho}

O presente trabalho está organizado como segue. No Capítulo 2 lidamos com dois problemas de empacotamento, um envolvendo minimizar a área de um poliedro que deve conter um conjunto dado de retângulos idênticos e outro que, dado um poliedro fixo, deve encontrar a maior quantidade de retângulos idênticos que podem ser empacotados. Consideramos modelos de programação disjuntiva e suas reformulações MIPs através da técnica do M-grande e do fecho convexo para os problemas em questão. Estudamos formas de eliminar soluções simétricas destes modelos, através da inserção de novas restrições ou da reformulação de restrições existentes. No Capítulo 3 apresentamos modelos de otimização para o problema de corte em dois estágios com sobras aproveitáveis. Baseados em [Lodi03] desenvolvemos dois modelos de otimização em dois níveis e os reformulamos em MIPs. Os dois modelos diferem essencialmente na forma de modelar itens do mesmo tipo ou idênticos. No primeiro modelo cada item é modelado individualmente, independentemente do fato de serem do mesmo tipo ou não. No segundo modelo apresentado, os itens do mesmo tipo são modelados de forma agrupada. No Capítulo 4 apresentamos os modelos de otimização para o problema genérico de corte de retângulos com sobras aproveitáveis. Modelamos as sobras aproveitáveis de duas formas diferentes: fixas nas extremidades superior e direita de um objeto e como dois itens artificiais de dimensões variáveis. Consideramos também duas diferentes formas de caracterizar se uma sobra é ou não aproveitável. Desenvolvemos quatro modelos de otimização em dois níveis e os reformulamos em MIPs. No Capítulo 5 apresentamos restrições de eliminação de simetrias para os modelos MIPs apresentados no Capítulo 4, bem como, uma estratégia alternativa para solução desses modelos. Finalmente, no Capítulo 6 apresentamos as conclusões, destacamos as contribuições deste trabalho e delineamos alguns possíveis trabalhos futuros. 


\section{Capítulo 2}

\section{Simetrias no empacotamento de retângulos em um poliedro}

O problema de empacotar a maior quantidade possível de retângulos idênticos dentro de uma região convexa arbitrária, permitindo que os retângulos sofram translações horizontais e verticais e possivelmente rotações de noventa graus tem sido explorado recentemente por diversos trabalhos [Birgin09, Birgin05a, Birgin06, Cassioli11, Birgin10b]. Quando a região convexa é um poliedro - diferentemente de quando a região é curva - o problema pode ser modelado como um problema de programação inteira mista (MIP - Mixed Integer Programming) e ser resolvido de forma ótima (veja [Beasley85b, Birgin10a, Birgin11, Birgin05b] para o caso particular de empacotar retângulos em retângulos). Quando todos os retângulos são idênticos, é possível definir soluções equivalentes, nas quais os papeis de um par de retângulos $R_{i}$ e $R_{j}$ são invertidos. A existência de diversas soluções equivalentes pode desacelerar um algoritmo do tipo branch-and-bound, na medida em que elas aumentam o tamanho da árvore de busca. Como resultado disso, diversos autores consideram "restrições de eliminação de simetrias" (symmetry-breaking constraints) que eliminam algumas dessas soluções equivalentes (veja, por exemplo, [Kallrath08, Lodi03]).

Neste capítulo, introduzimos três tipos de restrições de eliminação de simetrias: uma relacionada com as rotações dos retângulos e duas relacionadas à forma como os retângulos são ordenados espacialmente. Também analisamos experimentalmente a sua eficiência na redução da árvore de busca no contexto de algoritmos que usam o arcabouço branch-and-bound. Ilustramos o impacto das restrições de eliminação de simetrias no contexto de dois problemas: (i) empacotar a maior quantidade possível de retângulos idênticos em um triângulo equilátero e (ii) encontrar o menor triângulo equilátero no qual um conjunto de retângulos idênticos pode ser empacotado. Em ambos os problemas, os lados dos retângulos devem estar alinhados com os eixos cartesianos, ou seja, só podem sofrer rotações de noventa graus.

Um resultado um pouco surpreendente é que acrescentar restrições de eliminação de simetrias nem sempre é algo benéfico. Em particular, se alguém está tentando determinar se $k$ retângulos podem ser empacotados em um triângulo, e se a resposta for sim, então acrescentar restrições de eliminação de simetrias irá dificultar ou desacelerar a solução do MIP. A principal vantagem das restrições de eliminação de simetrias ocorre ao se provar a otimalidade de soluções ou a inviabilidade de um problema.

Este capítulo está organizado da seguinte maneira. A Seção 2.1 descreve os modelos de ambos os problemas. Na Seção 2.2, as restrições de eliminação de simetria são apresentadas. Apresentamos uma análise de experimentos numéricos na Seção 2.3 e, na Seção 2.4, algumas conclusões. 


\subsection{Modelos de programação inteira mista}

Considere o conjunto de retângulos $R_{1}, \ldots, R_{k}$ com altura $h$ e comprimento $w$, centrados na origem do espaço Cartesiano bidimensional com seus lados paralelos aos eixos. Desejamos empacotá-los em um dado poliedro $\Omega$ permitindo translações e rotações de noventa graus. Para modelar as rotações, introduzimos variáveis binárias

$$
r_{i} \in\{0,1\}, i=1, \ldots, k
$$

e definimos

$$
h_{i} \equiv\left(1-r_{i}\right) h+r_{i} w \text { e } w_{i} \equiv\left(1-r_{i}\right) w+r_{i} h, \quad i=1, \ldots, k,
$$

de forma que $r_{i}=0$ signifique que $h_{i}=h$ e $w_{i}=w$, i.e. que o retângulo $R_{i}$ preserva sua orientação original. Por outro lado, $r_{i}=1$ significa que $h_{i}=w$ e $w_{i}=h$, implicando que o retângulo $R_{i}$ foi rotacionado de noventa graus em relação à sua orientação original.

Seja $\left(c_{i}^{x}, c_{i}^{y}\right)^{T} \in \mathbb{R}^{2}$ as coordenadas do centro do retângulo $R_{i}$ com altura $h_{i}$ e comprimento $w_{i}$, para todo $i=1, \ldots, k$. Dois retângulos $R_{i}$ e $R_{j}$ não se sobrepõem, se e somente se

$$
\left|c_{i}^{x}-c_{j}^{x}\right| \geq \frac{1}{2}\left(w_{i}+w_{j}\right) \text { ou }\left|c_{i}^{y}-c_{j}^{y}\right| \geq \frac{1}{2}\left(h_{i}+h_{j}\right), \text { para todo } i=1, \ldots, k, j=i+1, \ldots, k .
$$

Acomodar os retângulos dentro de um objeto poliedral $\Omega$ pode ser modelado como

$$
v_{i}^{\ell} \in \Omega, \ell \in D, i=1, \ldots, k,
$$

onde $D=\{N W, N E, S E, S W\}$ e

$$
\begin{aligned}
v_{i}^{N W} & \equiv\left(c_{i}^{x}-\frac{1}{2} w_{i}, c_{i}^{y}+\frac{1}{2} h_{i}\right)^{T}, \quad v_{i}^{N E} \equiv\left(c_{i}^{x}+\frac{1}{2} w_{i}, c_{i}^{y}+\frac{1}{2} h_{i}\right)^{T}, \\
v_{i}^{S E} & \equiv\left(c_{i}^{x}+\frac{1}{2} w_{i}, c_{i}^{y}-\frac{1}{2} h_{i}\right)^{T}, \quad v_{i}^{S W} \equiv\left(c_{i}^{x}-\frac{1}{2} w_{i}, c_{i}^{y}-\frac{1}{2} h_{i}\right)^{T},
\end{aligned}
$$

definem os vértices de um retângulo $R_{i}$ para $i=1, \ldots, k$.

As restrições $(2.1,2.2,2.3)$ definem um modelo de programação linear disjuntivo generalizado (LGDP - linear generalized disjunctive programing) [Sawaya05] ${ }^{1}$, chamado $\mathcal{P}_{L G D P}^{k,[\Omega, h, w]}$ de agora em diante, para o problema de colocar $k$ retângulos sem sobreposição com altura $h$ e comprimento $w$, que podem diferir apenas por translações horizontais ou verticais e possivelmente por rotações de noventa graus, em $\Omega$.

$\mathrm{O}$ modelo possui $k$ variáveis binárias $\left(r_{i}, i=1, \ldots, k\right)$ e $2 k$ variáveis contínuas $\left(c_{i}^{x}\right.$ e $c_{i}^{y}$, $i=1, \ldots, k)$. Note que $h_{i}, w_{i}, v_{i}^{\ell}, \ell \in D, i=1, \ldots, k$, não são variáveis do modelo, mas valores auxiliares usados para simplificar a apresentação

Em (2.2) há $k(k-1) / 2$ restrições disjuntivas constituídas por quatro restrições lineares cada, enquanto que o número de restrições em (2.3) depende da descrição de $\Omega$. Por exemplo, se $\Omega$ descrevesse um triângulo, teríamos $12 k$ restrições lineares adicionais (três restrições lineares para cada um dos quatro vértices dos $k$ itens retangulares) Para resolver o problema original de empacotar a maior quantidade de retângulos possível, o problema $\mathcal{P}_{L G D P}^{k,[\Omega, h, w]}$ pode ser resolvido para valores crescentes de $k=1,2, \ldots$ até que o primeiro problema inviável $\mathcal{P}_{L G D P}^{k^{\prime},[\Omega, h, w]}$ seja detectado. Neste caso, $N=k^{\prime}-1$ é o maior número de retângulos que pode ser colocado em $\Omega$ e a solução de $\mathcal{P}_{L G D P}^{N,[\Omega, h, w]}$ mostra como alocar os retângulos em $\Omega$.

De acordo com [Sawaya05] (pp. 1892-1893), as alternativas mais comuns para reformular

\footnotetext{
${ }^{1}$ Consideramos que $|x| \geq c$ pode ser escrito como $x \geq c$ ou $x \leq-c$.
} 
as restrições disjuntivas que modelam a não sobreposição em (2.2) como restrições lineares com variáveis inteiras e contínuas são a reformulação do M-grande e a do fecho-convexo [Raman94, Balas98].

Definindo um par de variáveis $q_{i j}$ e $q_{j i}$ para cada par $(i, j)$ com $j>i$ e usando constantes $M_{w}>0$ e $M_{h}>0$ suficientemente grandes, a reformulação do M-grande de (2.2) pode ser escrita como

$$
\begin{aligned}
& c_{i}^{x}-c_{j}^{x} \geq \frac{1}{2}\left(w_{i}+w_{j}\right)-M_{w} q_{i j}-M_{w} q_{j i}, \\
& c_{j}^{x}-c_{i}^{x} \geq \frac{1}{2}\left(w_{i}+w_{j}\right)-M_{w}\left(1-q_{i j}\right)-M_{w}\left(1-q_{j i}\right), \\
& c_{i}^{y}-c_{j}^{y} \geq \frac{1}{2}\left(h_{i}+h_{j}\right)-M_{h}\left(1-q_{i j}\right)-M_{h} q_{j i}, \\
& c_{j}^{y}-c_{i}^{y} \geq \frac{1}{2}\left(h_{i}+h_{j}\right)-M_{h} q_{i j}-M_{h}\left(1-q_{j i}\right), \\
& q_{i j}, q_{j i} \in\{0,1\}, \text { para todo } i=1, \ldots, k, j=i+1, \ldots, k .
\end{aligned}
$$

Em (2.4), as variáveis $q_{i j}$ e $q_{j i}$ são usadas para garantir que pelo menos uma das quatro condições de não sobreposição seja satisfeita e podem ser interpretada através da posição relativa entre os retângulos $R_{i}$ e $R_{j}$. Grosseiramente falando, para os retângulos $R_{i}$ e $R_{j}$ não se sobreporem, existem quatro possibilidades: o retângulo $R_{j}$ pode estar à esquerda, à direita, acima ou abaixo do retângulo $R_{i}$; e essas possibilidades estão relacionadas respectivamente com (i) $q_{i j}=q_{j i}=0$, (ii) $q_{i j}=q_{j i}=1$, (iii) $q_{i j}=1$ e $q_{j i}=0$, (iv) $q_{i j}=0$ e $q_{j i}=1$. Veja a Figura 2.1.

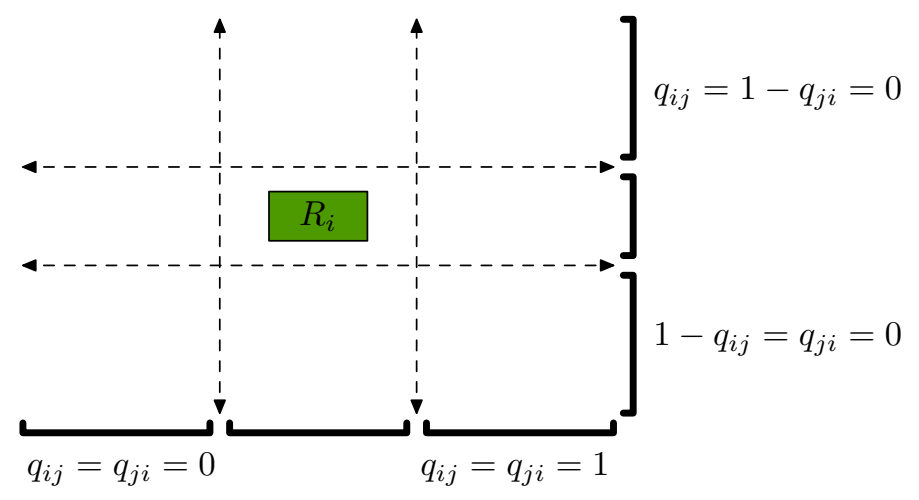

Figura 2.1: Relação entre os valores das variáveis binárias $q_{i j}$ e $q_{j i}$ em (2.4) e a posição relativa entre os retângulos $R_{i}$ e $R_{j}$.

Definindo dezesseis variáveis contínuas $d_{i,[i, j, m]}^{x}, d_{i,[i, j, m]}^{y}, d_{j,[i, j, m]}^{x}, d_{j,[i, j, m]}^{y}, m=1, \ldots, 4$, e quatro variáveis binárias $s_{[i, j, m]}, m=1, \ldots, 4$, para cada par $(i, j) \operatorname{com} j>i$, a reformulação do 
fecho-convexo de (2.2) pode ser escrita como

$$
\begin{aligned}
& \sum_{m=1}^{4} s_{[i, j, m]}=1, \quad s_{[i, j, m]} \in\{0,1\}, \quad m=1, \ldots, 4, \\
& c_{u}^{x}=\sum_{m=1}^{4} d_{u,[i, j, m]}^{x}, \quad c_{u}^{y}=\sum_{m=1}^{4} d_{u,[i, j, m]}^{y}, \quad u=i, j, \\
& 0 \leq d_{u,[i, j, m]}^{x} \leq \hat{c}^{x} s_{[i, j, m]}, \quad 0 \leq d_{u,[i, j, m]}^{y} \leq \hat{c}^{y} s_{[i, j, m]}, \quad u=i, j, \quad m=1, \ldots, 4, \\
& d_{i,[i, j, 1]}^{x}-d_{j,[i, j, 1]}^{x} \geq \frac{1}{2}\left(w_{i}+w_{j}\right) s_{[i, j, 1]}, \\
& d_{j,[i, j, 2]}^{x}-d_{i,[i, j, 2]}^{x} \geq \frac{1}{2}\left(w_{i}+w_{j}\right) s_{[i, j, 2]}, \\
& d_{i,[i, j, 3]}^{y}-d_{j,[i, j, 3]}^{y} \geq \frac{1}{2}\left(h_{i}+h_{j}\right) s_{[i, j, 3]}, \\
& d_{j,[i, j, 4]}^{y}-d_{i,[i, j, 4]}^{y} \geq \frac{1}{2}\left(h_{i}+h_{j}\right) s_{[i, j, 4]}, \\
& \text { para todo } i=1, \ldots, k, j=i+1, \ldots, k .
\end{aligned}
$$

Em (2.5), supomos que $0 \leq c_{i}^{x} \leq \hat{c}^{x}$ e $0 \leq c_{i}^{y} \leq \hat{c}^{y}$, para $i=1, \ldots, k$. Uma condição suficiente para se conseguir esses limitantes é

$$
\Omega \subseteq[0,0] \times\left[\hat{c}^{x}, \hat{c}^{y}\right] \subset \mathbb{R}^{2} .
$$

Suporemos que (2.6) vale e que as constantes $\hat{c}^{x}$ e $\hat{c}^{y}$ são conhecidas, de agora em diante. Se, por exemplo, $\Omega$ fosse um triângulo equilátero de lado $L$, seria suficiente posicioná-lo com um de seus lados paralelo ao eixo cartesiano horizontal, com seu vértice inferior esquerdo na origem e considerar $\hat{c}^{x} \equiv L$ e $\hat{c}^{y} \equiv(\sqrt{3} / 2) L$.

As restrições $(2.1,2.3,2.4)$ e $(2.1,2.3,2.5)$ definem modelos MIP, chamados $\mathcal{P}_{B M}^{k,[\Omega, h, w]}$ e $\mathcal{P}_{C H}^{k,[\Omega, h, w]}$, respectivamente, equivalentes à $\mathcal{P}_{L G D P}^{k,[\Omega, h, w]} . \operatorname{Em} \mathcal{P}_{B M}^{k,[\Omega, h, w]}$ há duas variáveis binárias adicionais $\left(q_{i j}\right.$, $\left.q_{j i}, j=i+1, \ldots, k\right)$ para cada par de retângulos, com um total de $k+2[k(k-1) / 2]=k^{2}$ variáveis binárias e $2 k$ variáveis contínuas. A quantidade de restrições em $(2.4)$ é $4 k(k-1) / 2$. Em $\mathcal{P}_{C H}^{k,[\Omega, h, w]}$ há dezesseis variáveis contínuas adicionais $\left(d_{i,[i, j, m]}^{x}, d_{i,[i, j, m]}^{y}, d_{j,[i, j, m]}^{x}, d_{j,[i, j, m]}^{y}, m=1, \ldots, 4\right)$ para cada par de retângulos, com um total de $2 k+16[k(k-1) / 2]$ variáveis contínuas. Há também quatro variáveis binárias adicionais $\left(s_{[i, j, m]}, m=1, \ldots, 4\right)$ para cada par de retângulos, com um total de $k+4[k(k-1) / 2]=2 k^{2}-k$ variáveis binárias. A quantidade de restrições em (2.5) é $41 k(k-1) / 2$.

Suponhamos agora que $\Omega$ é um poliedro dado por $\Omega \equiv\left\{x \in \mathbb{R}^{2} \mid A x \leq b\right\}$ e que um número fixo $k$ de retângulos idênticos é dado. O problema de encontrar o "menor poliedro do mesmo tipo de $\Omega$ " que acomode os $k$ retângulos, pode ser modelado como

$$
\begin{array}{ll}
\text { Minimizar } & L \\
\text { s.a } & A v_{i}^{\ell} \leq L b, \ell \in D, i=1, \ldots, k, \\
& \text { e as restrições }(2.1,2.2) .
\end{array}
$$

As reformulações MIP através da técnica do fecho-convexo e do M-grande de (2.7) podem ser obtidas, substituindo (2.2) por (2.5) e (2.4), respectivamente. Chamaremos o modelo LGDP (2.7), sua reformulação MIP usando a técnica do M-grande e a sua reformulação MIP usando a técnica do fecho-convexo de $\mathcal{S}_{L G D P}^{k,[\Omega, h, w]}, \mathcal{S}_{B M}^{k,[\Omega, h, w]}$ e $\mathcal{S}_{C H}^{k,[\Omega, h, w]}$, respectivamente. 


\subsection{Restrições de eliminação de simetrias}

Dada uma solução $\left(c_{i}^{x}, c_{i}^{y}, r_{i}, i=1, \ldots, k\right)$ para o problema $\mathcal{P}_{L G D P}^{k,[\Omega, h, w]}$ e escolhendo um par $(i, j)$ com $j>i$, é possível definir uma solução equivalente ou simétrica na qual os papéis dos retângulos $R_{i}$ e $R_{j}$ são intercambiados. De agora em diante usaremos os termos soluções equivalentes ou soluções simétricas para nos referir a soluções que podem ser obtidas uma a partir da outra por uma simples renumeração dos retângulos empacotados. É fácil ver que cada solução pertence a uma família composta por $k$ ! soluções equivalentes, correspondendo a todas as possíveis formas de se numerar os retângulos. É bem conhecido que a presença de soluções simétricas pode prejudicar a performance de um método do tipo branch-and-bound (veja, por exemplo, [Ostrowski10] e demais referências lá mencionadas). Basicamente, um método do tipo branchand-bound tradicional pode ter dificuldades em podar nós da árvore associados com subproblemas equivalentes e, em consequência, uma enumeração proibitivamente grande pode ser necessária.

O conjunto mais simples de restrições que pode ser acrescentado para eliminar soluções equivalentes, preservando pelo menos um representante no conjunto viável, é:

$$
r_{i} \leq r_{i+1}, i=1, \ldots, k-1
$$

Se houver uma solução com $p$ retângulos não rotacionados e $k-p$ rotacionados, o conjunto de restrições (2.8) claramente diz que os retângulos não rotacionados devem ser os retângulos $R_{1}, \ldots, R_{p}$ enquanto que os retângulos rotacionados devem ser os retângulos $R_{p+1}, \ldots, R_{k}$.

Entretanto, ao considerar o modelo $\mathcal{P}_{L G D P}^{k,[\Omega, h, w]}$ com as restrições adicionais (2.8), soluções simétricas que correspondem ao intercâmbio de lugar de qualquer par de retângulos com a mesma orientação ainda existem. Para eliminar soluções simétricas deste tipo, gostaríamos de acrescentar restrições que imponham uma ordem lexicográfica (total) entre os retângulos com a mesma orientação. Essas restrições podem ser escritas como

$$
r_{i}=r_{i+1} \Rightarrow\left(c_{i+1}^{x}>c_{i}^{x} \text { ou }\left(c_{i+1}^{x}=c_{i}^{x} \text { e } c_{i+1}^{y} \geq c_{i}^{y}\right)\right), i=1, \ldots, k-1,
$$

mas a desigualdade estrita em (2.9) e a continuidade das variáveis $c_{i}^{x}, c_{i}^{y}, i=1, \ldots, k$, nos impedem de expressar a ordem lexicográfica como restrições de um modelo de programação inteira mista. Substituir a desigualdade estrita por um "menor ou igual" reduz (2.9) para:

$$
r_{i}=r_{i+1} \Rightarrow c_{i+1}^{x} \geq c_{i}^{x}, i=1, \ldots, k-1,
$$

o que é na verdade uma pré-ordem (relação binária reflexiva e transitiva) entre os retângulos com a mesma orientação. De fato, para qualquer $\alpha, \beta \in \mathbb{R}$,

$$
r_{i}=r_{i+1} \Rightarrow \alpha c_{i+1}^{x}+\beta c_{i+1}^{y} \geq \alpha c_{i}^{x}+\beta c_{i}^{y}, i=1, \ldots, k-1,
$$

ou, equivalentemente,

$$
\alpha c_{i+1}^{x}+\beta c_{i+1}^{y} \geq \alpha c_{i}^{x}+\beta c_{i}^{y}-M_{o}\left(r_{i+1}-r_{i}\right), i=1, \ldots, k-1,
$$

onde $M_{o}>0$ é uma constante suficientemente grande, define uma pré-ordem entre os retângulos com a mesma orientação. A Figura 2.2 ilustra o efeito das restrições de eliminação de simetria (2.8) e (2.10).

Podemos também obter outra forma de eliminar soluções simétricas substituindo as restrições 


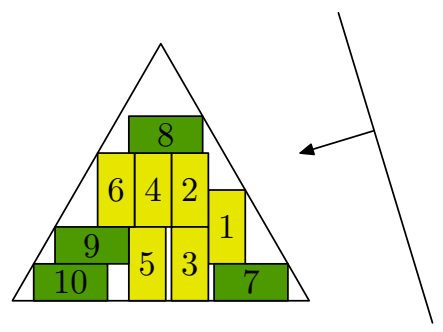

Figura 2.2: Esta figura corresponde à representação gráfica de uma instância com 10 itens retangulares. A linha representa a restrição (2.10) com $\alpha \approx-0.827$ e $\beta \approx-0.251$. Note que quando a linha é arrastada na direção indicada pela seta, retângulos com a mesma orientação são numerados em ordem crescente à medida que a linha toca em seus centros. Isso ilustra a pré-ordem imposta pela restrição (2.10). Adicionalmente, os retângulos $R_{i}$ não rotacionados, que correspondem a $r_{i}=0$, são numerados antes dos rotacionados $\left(r_{i}=1\right)$, conforme imposto pela restrição (2.8).

de não sobreposição (2.2) por:

$$
c_{j}^{x}-c_{i}^{x} \geq \frac{1}{2}\left(w_{i}+w_{j}\right) \text { ou } c_{j}^{y}-c_{i}^{y} \geq \frac{1}{2}\left(h_{i}+h_{j}\right), \text { para todo } i=1, \ldots, k, j=i+1, \ldots, k .
$$

As restrições (2.11) pedem que para cada par $(i, j) \operatorname{com} j>i, R_{j}$ e $R_{i}$ não se sobreponham e que $R_{j}$ esteja posicionado à direita ou acima de $R_{i}$.

Assim como (2.2), as restrições disjuntivas (2.11) podem ser reescritas como um conjunto de restrições MIP considerando as reformulações do M-grande ou do fecho-convexo. Definindo uma variável binária $q_{i j}$ para cada par $(i, j)$ com $j>i$, e usando constantes $M_{w}>0$ e $M_{h}>0$ suficientemente grandes, a reformulação do M-grande de (2.11) pode ser escrita como:

$$
\begin{aligned}
& c_{j}^{x}-c_{i}^{x} \geq \frac{1}{2}\left(w_{i}+w_{j}\right)-M_{w} q_{i j}, \\
& c_{j}^{y}-c_{i}^{y} \geq \frac{1}{2}\left(h_{i}+h_{j}\right)-M_{h}\left(1-q_{i j}\right), \\
& q_{i j} \in\{0,1\}, \text { para todo } i=1, \ldots, k, j=i+1, \ldots, k .
\end{aligned}
$$

Em (2.12), $q_{i j}$ é utilizada para que pelo menos uma das duas restrições seja imposta e, da mesma forma que em (2.4), pode ser interpretada como capturando a posição relativa entre os retângulos $R_{i}$ e $R_{j}$, sendo que $q_{i j}=0$ significa que $R_{j}$ está à direita de $R_{i}$ e $q_{i j}=1$ significa que $R_{j}$ està acima de $R_{i}$. Definindo oito variáveis contínuas $d_{i,[i, j, m]}^{x}, d_{i,[i, j, m]}^{y}, d_{j,[i, j, m]}^{x}, d_{j,[i, j, m]}^{y}, m=1,2$, e duas variáveis binárias $s_{[i, j, 1]}$ e $s_{[i, j, 2]}$, para cada $\operatorname{par}(i, j) \operatorname{com} j>i$, a reformulação do fecho-convexo 
de (2.11) pode ser escrita como:

$$
\begin{aligned}
& s_{[i, j, 1]}+s_{[i, j, 2]}=1, \quad s_{[i, j, 1]}, s_{[i, j, 2]} \in\{0,1\}, \\
& c_{u}^{x}=d_{u,[i, j, 1]}^{x}+d_{u,[i, j, 2]}^{x}, \quad c_{u}^{y}=d_{u,[i, j, 1]}^{y}+d_{u,[i, j, 2]}^{y}, \quad u=i, j, \\
& 0 \leq d_{u,[i, j, 1]}^{x} \leq \hat{c}^{x} s_{[i, j, 1]}, \quad 0 \leq d_{u,[i, j, 1]}^{y} \leq \hat{c}^{y} s_{[i, j, 1]}, \quad u=i, j, \\
& 0 \leq d_{u,[i, j, 2]}^{x} \leq \hat{c}^{x} s_{[i, j, 2]}, \quad 0 \leq d_{u,[i, j, 2]}^{y} \leq \hat{c}^{y} s_{[i, j, 2]}, \quad u=i, j, \\
& d_{j,[i, j, 1]}^{x}-d_{i,[i, j, 1]}^{x} \geq \frac{1}{2}\left(w_{i}+w_{j}\right) s_{[i, j, 1]}, \\
& d_{j,[i, j, 2]}^{y}-d_{i,[i, j, 2]}^{y} \geq \frac{1}{2}\left(h_{i}+h_{j}\right) s_{[i, j, 2]}, \\
& \text { para todo } i=1, \ldots, k, j=i+1, \ldots, k .
\end{aligned}
$$

Resumindo, adicionando as restrições $(2.8,2.10)$ aos modelos $\mathcal{P}_{L G D P}^{k,[\Omega, h, w]}, \mathcal{P}_{B M}^{k,[\Omega, h, w]}$, e $\mathcal{P}_{C H}^{k,[\Omega, h, w]}$, obtemos modelos equivalentes, que serão chamados de $\mathcal{Q}_{L G D P}^{k,[\Omega, h, w]}, \mathcal{Q}_{B M}^{k,[\Omega, h, w]}$, e $\mathcal{Q}_{C H}^{k,[\Omega, h, w]}$, respectivamente. Por outro lado, substituindo (2.2), (2.4) e (2.5), por (2.11), (2.12) e (2.13), em $\mathcal{P}_{L G D P}^{k,[\Omega, h, w]}$, $\mathcal{P}_{B M}^{k,[\Omega, h, w]}$ e $\mathcal{P}_{C H}^{k,[\Omega, h, w]}$, também obtemos modelos equivalentes que serão chamados de $\mathcal{R}_{L G D P}^{k,[\Omega, h, w]}$, $\mathcal{R}_{B M}^{k,[\Omega, h, w]}$, e $\mathcal{R}_{C H}^{k,[\Omega, h, w]}$, respectivamente. Os modelos “ $\mathcal{Q}$ ” e “ $\mathcal{R}$ ” são equivalentes aos seus correspondentes modelos " $\mathcal{P}$ ", mas com menos representantes de cada família de soluções equivalentes ou simétricas. A Figura 2.3 resume as principais características de cada modelo e as relações entre eles. Para ver que pelo menos um representante de cada família de soluções simétricas para um modelo "P " é também uma solução viável para os modelos " $\mathcal{Q}$ " e " $\mathcal{R}$ " correspondentes, basta notar que dada uma solução para um modelo " $\mathcal{P}$ " é possível renumerar os retângulos de forma a satisfazer as restrições adicionais dos modelos " $\mathcal{Q}$ " e " $\mathcal{R}$ ".

De forma análoga, as restrições de eliminação de simetria podem também ser facilmente incorporadas nos modelos $\mathcal{S}_{L G D P}^{k,[\Omega, h, w]}, \mathcal{S}_{B M}^{k,[\Omega, h, w]}$ e $\mathcal{S}_{C H}^{k,[\Omega, h, w]}$. Chamaremos de $\mathcal{T}_{L G D P}^{k,[\Omega, h, w]}, \mathcal{T}_{B M}^{k,[\Omega, h, w]}$ e $\mathcal{T}_{C H}^{k,[\Omega, h, w]}$ os modelos resultantes da adição das restrições de eliminação de simetria (2.8,2.10); enquanto chamamos de $\mathcal{U}_{L G D P}^{k,[\Omega, h, w]}, \mathcal{U}_{B M}^{k,[\Omega, h, w]}$, e $\mathcal{U}_{C H}^{k,[\Omega, h, w]}$ os modelos que resultam da substituição de (2.2), (2.4) e (2.5), por (2.11), (2.12) e (2.13), em $\mathcal{S}_{L G D P}^{k,[\Omega, h, w]}, \mathcal{S}_{B M}^{k,[\Omega, h, w]}$ e $\mathcal{S}_{C H}^{k,[\Omega, h, w]}$, respectivamente.

\subsection{Experimentos numéricos}

Nos experimentos numéricos, consideramos que os objetos são triangulos equiláteros e os itens são retangulos de altura $h=1$ e comprimento $w=0.5$. A escolha por triângulos equiláteros, em nossos experimentos ilustrativos, foi arbitrária e não há qualquer dificuldade adicional em considerar um objeto poliedral genérico $\Omega$, além do fato de ter que lidar com as restrições lineares adicionais necessárias para definir o poliedro. Note que, como detalhado em (2.3), se o poliedro for definido por $p$ desigualdades lineares, cada item requer somente $4 p$ restrições lineares para impor que seus vértices sejam acomodados no objeto. Todos os modelos considerados nos experimentos numéricos foram implementados em $\mathrm{C} / \mathrm{C}++$ (gcc versão 4.4.3) e resolvidos com CPLEX (IBM ILOG AMPL/CPLEX 12.1.0) usando seus parâmetros padrão. Isto significa que uma solução é considerada ótima pelo solver quando:

$$
G A P \text { absoluto }=\text { melhor solução viável }- \text { melhor limitante inferior } \leq 10^{-6}
$$




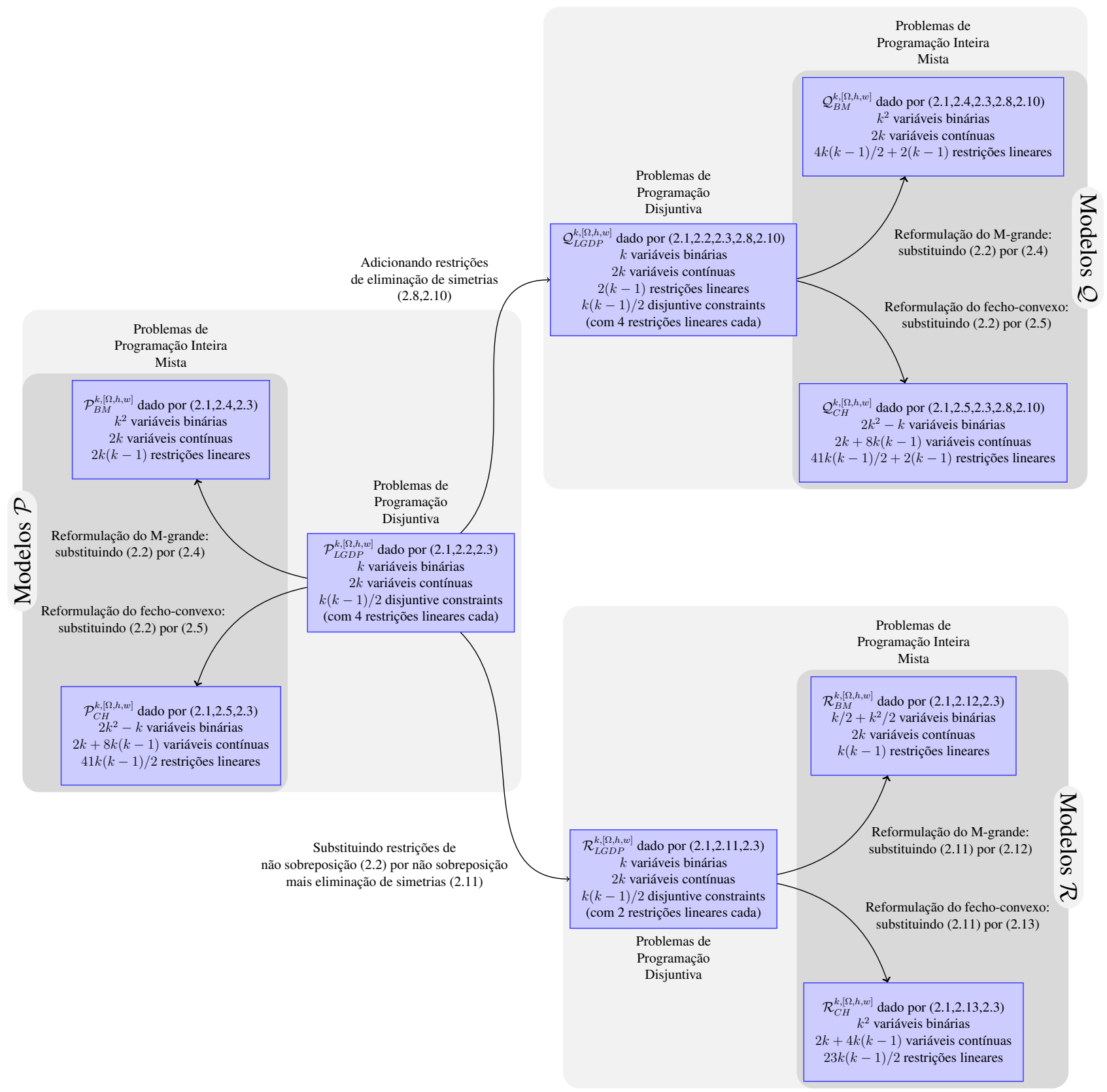

Figura 2.3: Descrição dos modelos (de viabilidade) de programação disjuntiva e programação inteiramista para o problema de empacotar $k$ retângulos idênticos, com altura he comprimento $w$, em um poliedro $\Omega$, permitindo rotações de noventa graus e translações dos itens. As quantidades de restrições não incluem as restrições do tipo (2.3), que dependem da descrição de $\Omega$. 
ou

$$
G A P \text { relativo }=\frac{\mid \text { melhor solução viável }- \text { melhor limitante inferior } \mid}{10^{-10}+\mid \text { melhor solução viável } \mid} \leq 10^{-4} .
$$

Para testar os modelos propostos, valores para as constantes $\alpha, \beta$ e $M_{o}$ em (2.10), $M_{w}$ e $M_{h}$ em $(2.4,2.12)$, e $\hat{c}^{x}$ e $\hat{c}^{y}$ em $(2.5,2.13)$ devem ser dados. Escolhemos, para $\alpha$ e $\beta$, valores aleatórios com distribuição uniforme no intervalo $[-1,1]$. Para $M_{o}$, basta escolher um valor tal que $M_{o} \geq L_{u b}+\frac{\sqrt{3}}{2} L_{u b}$, onde $L_{u b}$ é um limitante superior para o (possivelmente desconhecido) lado $L$ do triângulo equilátero. Quanto a $M_{w}$ e $M_{h}$, basta escolher um valor tal que $M_{w} \geq L_{u b}$ e $M_{h} \geq \frac{\sqrt{3}}{2} L_{u b}$. Valores válidos para $\hat{c}^{x}$ and $\hat{c}^{y}$ em $(2.5,2.13)$ são $\hat{c}^{x}=L_{u b}$ e $\hat{c}^{y}=\frac{\sqrt{3}}{2} L_{u b}$. Quando o triângulo é dado e o problema consiste em computar a maior quantidade de retângulos que pode ser empacotada no triângulo, $L$ é conhecido e definimos $L_{u b}=L$. Quando o problema consiste em computar o menor triângulo equilátero que empacota uma dada quantidade $N$ de retângulos fixa, procedemos da seguinte forma. O lado de um quadrado que contenha os $N$ retângulos idênticos é calculado e o lado do menor triângulo equilátero que circunscreva esse quadrado é tomado como $L_{u b}$. Como resultado, no caso em que $L$ é desconhecido, os valores considerados para $L_{u b}$ foram $L_{u b}=2.16$ para $N \in\{1,2\}, L_{u b}=4.31$ para $N \in\{2,3, \ldots, 8\}$ e $L_{u b}=6.47$ para $N \in\{9,10, \ldots, 16\}$. Note que a escolha destes valores é importante já que é sabido que eles interferem na performance do solver MIP considerado.

\subsubsection{Minimizando a dimensão do objeto}

Nesta subseção lidamos com o problema de encontrar o menor triângulo equilátero que pode acomodar um conjunto dado de $N$ itens retangulares idênticos (lembre-se que estamos lidando com itens retangulares com altura $h=1$ e comprimento $w=0.5$ ).

Inicialmente, em um conjunto pequeno de experimentos, a fim de ter um ponto de comparação, tentamos resolver instâncias de $\mathcal{S}_{L G D P}^{k,[\Omega, h, w]}$ (modelo LGDP (2.7)), $\mathcal{S}_{B M}^{k,[\Omega, h, w]}$ (reformulação MIP com M-grande) e $\mathcal{S}_{C H}^{k,[\Omega, h, w]}$ (reformulação MIP com fecho-convexo), que não incluem qualquer restrição de eliminação de simetrias, aumentando a quantidade de itens $N \in\{1,2, \ldots\}$. A Tabela 2.1 contém os resultados. Na tabela, $N$ é a quantidade de itens a ser empacotada. Para o lado do menor triângulo equilátero que empacota os $N$ itens, a tabela informa o melhor limitante inferior, bem como, a melhor solução viável encontrada pelo solver. Também são fornecidos os GAPs absoluto e relativo. O lado direito da tabela mostra as medidas de esforço: "MIP" é a quantidade de iterações MIP, "Nós B\&B" é a quantidade de nós na árvore branch-and-bound e "Tempo de CPU" é o tempo de CPU em segundos. Na tabela, podemos ver que com um limite de tempo de CPU de seis horas, não pudemos resolver instâncias com $N>8$.

Em um segundo conjunto de experimentos testamos a eficiência das restrições de eliminação de simetrias $(2.8,2.10)$. Assim, consideramos instâncias dos problemas $\mathcal{T}_{L G D P}^{k,[\Omega, h, w]}$ (modelo LGDP (2.7) mais restrições de eliminação de simetrias $(2.8,2.10)$ ), $\mathcal{T}_{B M}^{k,[\Omega, h, w]}$ (reformulação MIP com M-grande), e $\mathcal{T}_{C H}^{k,[\Omega, h, w]}$ (reformulação MIP através do fecho-convexo). Em relação à restrição (2.8), é fácil ver que há somente $k+1$ combinações possíveis de valores para as variáveis $r_{i}$, $i=1, \ldots, k$, tais que (2.8) vale. As $k+1$ possibilidades correspondem a

$$
r_{i}=\left\{\begin{array}{ll}
0, & \text { if } i \leq p, \\
1, & \text { caso contrário, }
\end{array} \quad i=1, \ldots, k,\right.
$$

para $p=0, \ldots, k$. Assim, há duas formas possíveis de considerar a restrição (2.8) em relação ao problema (2.7): (i) $k+1$ problemas do tipo (2.7) podem ser resolvidos fixando $r_{i}, i=1, \ldots, k$, como sugerido em (2.14) para $p=0, \ldots, k$, ou (ii) adicionar a equação (2.8) em (2.7) resolvendo $(2.7,2.8)$. As Tabelas 2.2 e 2.3 mostram as opões (i) e (ii), respectivamente, com a adição da res- 


\begin{tabular}{|c|c|c|c|c|c|c|c|c|}
\hline \multicolumn{6}{|c|}{ Dados da instância } & \multicolumn{3}{|c|}{ Medidas de esforço } \\
\hline & \multirow{2}{*}{$N$ fixo } & \multicolumn{2}{|c|}{ Lado do triângulo } & \multicolumn{2}{|c|}{ GAP MIP } & \multirow{2}{*}{ MIP } & \multirow{2}{*}{ Nós $B \& B$} & \multirow{2}{*}{ Tempo de CPU } \\
\hline & & Melhor limitante inferior & Melhor solução viável & Relativo & Absoluto & & & \\
\hline \multirow{10}{*}{ 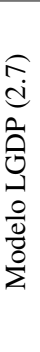 } & 1 & 1,577350269189626 & 1,577350269189626 & $0,00 \mathrm{e}+00$ & $0,00 \mathrm{e}+00$ & 0 & 0 & 0,00 \\
\hline & 2 & 2,154700538379251 & 2,154700538379251 & $0,00 \mathrm{e}+00$ & $0,00 \mathrm{e}+00$ & 38 & 4 & 0,01 \\
\hline & 3 & 2,443375672974064 & 2,443375672974064 & $0,00 \mathrm{e}+00$ & $0,00 \mathrm{e}+00$ & 215 & 53 & 0,01 \\
\hline & 4 & 2,732050807568878 & 2,732050807568878 & $0,00 \mathrm{e}+00$ & $0,00 \mathrm{e}+00$ & 1.212 & 315 & 0,04 \\
\hline & 5 & 3,020725942163691 & 3,020725942163691 & $0,00 \mathrm{e}+00$ & $0,00 \mathrm{e}+00$ & 41.003 & 12.476 & 1,46 \\
\hline & 6 & 3,231852460094526 & 3,232050807568877 & $6,14 \mathrm{e}-05$ & $1,98 \mathrm{e}-04$ & 910.795 & 232.853 & 40,26 \\
\hline & 7 & 3,443060783087007 & 3,443375672338170 & $9,14 \mathrm{e}-05$ & $3,15 \mathrm{e}-04$ & $27.536,014$ & $6.626,931$ & $1.466,85$ \\
\hline & 8 & 3,597717089329720 & 3,598076211353316 & $9,98 \mathrm{e}-05$ & $3,59 \mathrm{e}-04$ & $153.247,823$ & $36.600,213$ & $11.186,81$ \\
\hline & 9 & 3,215704772343326 & 3,809401068449104 & $1,56 \mathrm{e}-01$ & $5,94 \mathrm{e}-01$ & - & - & $>21.600,00$ \\
\hline & 10 & 3,016083139658178 & 4,020725924957480 & $2,50 \mathrm{e}-01$ & $1,00 \mathrm{e}+00$ & - & - & $>21.600,00$ \\
\hline \multirow{10}{*}{ 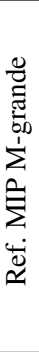 } & 1 & 1,577350269189626 & 1,577350269189626 & $0,00 \mathrm{e}+00$ & $0,00 \mathrm{e}+00$ & 0 & 0 & 0,00 \\
\hline & 2 & 2,154700538379251 & 2,154700538379251 & $0,00 \mathrm{e}+00$ & $0,00 \mathrm{e}+00$ & 30 & 11 & 0,01 \\
\hline & 3 & 2,443375672974065 & 2,443375672974065 & $0,00 \mathrm{e}+00$ & $0,00 \mathrm{e}+00$ & 149 & 80 & 0,01 \\
\hline & 4 & 2,732050807568878 & 2,732050807568878 & $0,00 \mathrm{e}+00$ & $0,00 \mathrm{e}+00$ & 1.315 & 725 & 0,07 \\
\hline & 5 & 3,020725942163690 & 3,020725942163690 & $0,00 \mathrm{e}+00$ & $0,00 \mathrm{e}+00$ & 28.832 & 17.763 & 1,08 \\
\hline & 6 & 3,232050807568878 & 3,232050807568878 & $0,00 \mathrm{e}+00$ & $0,00 \mathrm{e}+00$ & 457.542 & 283.835 & 17,45 \\
\hline & 7 & 3,443375672974065 & 3,443375672974065 & $0,00 \mathrm{e}+00$ & $0,00 \mathrm{e}+00$ & $27.097,399$ & $14.284,652$ & $1.032,67$ \\
\hline & 8 & 3,443375672974065 & 3,598076205466564 & $4,30 \mathrm{e}-02$ & $1,55 \mathrm{e}-01$ & - & - & $>21.600,00$ \\
\hline & 9 & 3,154700538379261 & 3,809401070449102 & $1,72 \mathrm{e}-01$ & $6,55 \mathrm{e}-01$ & - & - & $>21.600,00$ \\
\hline & 10 & 3,065384140902228 & 4,020725935431639 & $2,38 \mathrm{e}-01$ & $9,55 \mathrm{e}-01$ & - & - & $>21.600,00$ \\
\hline \multirow{10}{*}{ 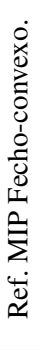 } & 1 & 1,577350269189626 & 1,577350269189626 & $0,00 \mathrm{e}+00$ & $0,00 \mathrm{e}+00$ & 0 & 0 & 0,00 \\
\hline & 2 & 2,154700518676984 & 2,154700529884166 & $5,20 \mathrm{e}-09$ & $1,12 \mathrm{e}-08$ & 78 & 16 & 0,02 \\
\hline & 3 & 2,443375667541825 & 2,443375667541825 & $0,00 \mathrm{e}+00$ & $0,00 \mathrm{e}+00$ & 2.123 & 259 & 0,07 \\
\hline & 4 & 2,732031213559349 & 2,732050717264398 & $7,14 \mathrm{e}-06$ & $1,95 \mathrm{e}-05$ & 33.886 & 3.841 & 1,16 \\
\hline & 5 & 3,020486516477845 & 3,020725894730525 & $7,92 \mathrm{e}-05$ & $2,39 \mathrm{e}-04$ & $1.592,352$ & 138.176 & 60,18 \\
\hline & 6 & 3,231727579601285 & 3,232050397095424 & $9,99 \mathrm{e}-05$ & $3,23 \mathrm{e}-04$ & $45.750,048$ & $3.594,324$ & $2.019,11$ \\
\hline & 7 & 3,154699924971216 & 3,443373062238137 & $8,38 \mathrm{e}-02$ & $2,89 \mathrm{e}-01$ & - & - & $>21.600,00$ \\
\hline & 8 & 2,809401076681599 & 3,598076080375179 & $2,19 \mathrm{e}-01$ & $7,89 \mathrm{e}-01$ & - & - & $>21.600,00$ \\
\hline & 9 & 2,618740741071466 & 3,866023671044396 & $3,23 \mathrm{e}-01$ & $1,25 \mathrm{e}+00$ & - & - & $>21.600,00$ \\
\hline & 10 & 2,427524391209028 & 4,020724784525934 & $3,96 \mathrm{e}-01$ & $1,59 \mathrm{e}+00$ & - & - & $>21.600,00$ \\
\hline
\end{tabular}

Tabela 2.1: Instâncias do modelo $\mathcal{S}_{L G D P}^{k,[\Omega, h, w]}$ e suas reformulações $M I P \mathcal{S}_{B M}^{k,[\Omega, h, w]}$ e $\mathcal{S}_{C H}^{k,[\Omega, h, w]}$ com quantidades crescentes de itens $N \in\{1,2, \ldots\}$. Lembrando que estes modelos correspondem aos modelos puros, sem restrições de eliminação de simetria.

trição (2.10). Na Tabela 2.2, podemos ver que todas as instâncias de $\mathcal{T}_{L G D P}^{k,[\Omega, h, w]}$ e $\mathcal{T}_{B M}^{k,[\Omega, h, w]}$ com $N$ até 10 são resolvidas com otimalidade, enquanto instâncias de $\mathcal{T}_{C H}^{k,[\Omega, h, w]}$ são resolvidas com otimalidade somente para $N$ até $8 .^{2}$ De qualquer forma, nos três modelos, as restrições de eliminação de simetrias $(2.8,2.10)$ ajudaram a reduzir o esforço computacional necessário para resolver as instâncias. Em particular, note que o tempo de CPU usado para resolver as instâncias com $N=8$, a maior instância resolvida sem a ajuda das restrições de eliminação de simetrias, foi reduzido de 11186,81 segundos (veja a Tabela 2.1) para 16,05 segundos (veja a Tabela 2.2). A comparação entre as Tabelas 2.2 e 2.3 mostra que parece mais vantajoso usar a estratégia (i) ao invés da estratégia (ii) para tratar com as restrições (2.8). As Tabelas 2.1-2.3 sugerem que o solver lida mais eficientemente com o modelo LGDP original do que com suas reformulações MIP. Por outro lado, as reformulações através da técnica do M-grande podem ser resolvidas com menos esforço do que as reformulações usando a técnica do fecho-convexo.

Para completar os experimentos desta subseção, testamos a eficiência de substituir, no modelo LGDP (2.7), as restrições de não sobreposição (2.2) pelas restrições de não sobreposição mais as restrições de eliminação de simetrias (2.11). Para tanto, experimentamos resolver instâncias do modelo LGDP $\mathcal{U}_{L G D P}^{k,[\Omega, h, w]}$ e suas reformulações MIP $\mathcal{U}_{B M}^{k,[\Omega, h, w]}$ e $\mathcal{U}_{C H}^{k,[\Omega, h, w]}$, para um número crescente de itens $N \in\{1,2, \ldots\}$.

\footnotetext{
${ }^{2}$ Vale mencionar que para $N=9$ o menor $G A P$ reportado corresponde à solução para o caso $r_{1}=0$ e $r_{i}=1, i>$ 1 , e que não houve tempo suficiente para considerar a maior parte das combinações viáveis para um dado valor das variáveis de rotação $r_{i}$ considerando-se os limites impostos.
} 


\begin{tabular}{|c|c|c|c|c|c|c|c|c|}
\hline \multicolumn{6}{|c|}{ Dados da instância } & \multicolumn{3}{|c|}{ Medidas de esforço } \\
\hline & \multirow{2}{*}{$N$ fixo } & \multicolumn{2}{|c|}{ Lado do Triângulo } & \multicolumn{2}{|c|}{ GAP MIP } & \multirow{2}{*}{ MIP } & \multirow{2}{*}{ Nós B\&B } & \multirow{2}{*}{ Tempo de CPU } \\
\hline & & Melhor limitante inferior & Melhor solução viável & Relativo & Absoluto & & & \\
\hline \multirow{10}{*}{ 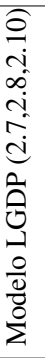 } & 1 & 1,577350269189625 & 1,577350269189625 & $0,00 \mathrm{e}+00$ & $0,00 \mathrm{e}+00$ & 0 & 0 & 0,01 \\
\hline & 2 & 2,154700538379168 & 2,154700538379168 & $0,00 \mathrm{e}+00$ & $0,00 \mathrm{e}+00$ & 0 & 0 & 0,00 \\
\hline & 3 & 2,443375672974064 & 2,443375672974064 & $0,00 \mathrm{e}+00$ & $0,00 \mathrm{e}+00$ & 143 & 16 & 0,02 \\
\hline & 4 & 2,732050807568876 & 2,732050807568876 & $0,00 \mathrm{e}+00$ & $0,00 \mathrm{e}+00$ & 957 & 170 & 0,06 \\
\hline & 5 & 3,020725942163691 & 3,020725942163691 & $0,00 \mathrm{e}+00$ & $0,00 \mathrm{e}+00$ & 7.787 & 1.879 & 0,28 \\
\hline & 6 & 3,232050807568879 & 3,232050807568879 & $0,00 \mathrm{e}+00$ & $0,00 \mathrm{e}+00$ & 40.757 & 7.211 & 1,41 \\
\hline & 7 & 3,443375672974065 & 3,443375672974064 & $2,58 \mathrm{e}-16$ & $8,88 \mathrm{e}-16$ & 90.126 & 17.075 & 3,31 \\
\hline & 8 & 3,598076211353316 & 3,598076211353316 & $0,00 \mathrm{e}+00$ & $0,00 \mathrm{e}+00$ & 423.122 & 67.662 & 16,05 \\
\hline & 9 & 3,809162326070802 & 3,809401076758502 & $6,27 \mathrm{e}-05$ & $2,39 \mathrm{e}-04$ & $14.817,741$ & $2.466,016$ & 590,69 \\
\hline & 10 & 4,020399584377308 & 4,020725942163673 & $8,12 \mathrm{e}-05$ & $3,26 \mathrm{e}-04$ & $36.412,075$ & $4.894,328$ & $1.680,93$ \\
\hline \multirow{10}{*}{ 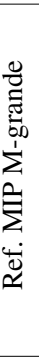 } & 1 & 1,577350269189626 & 1,577350269189626 & $0,00 \mathrm{e}+00$ & $0,00 \mathrm{e}+00$ & 0 & 0 & 0,01 \\
\hline & 2 & 2,154700538379251 & 2,154700538379251 & $0,00 \mathrm{e}+00$ & $0,00 \mathrm{e}+00$ & 0 & 0 & 0,00 \\
\hline & 3 & 2,443375672974064 & 2,443375672974064 & $0,00 \mathrm{e}+00$ & $0,00 \mathrm{e}+00$ & 166 & 76 & 0,02 \\
\hline & 4 & 2,732050807568878 & 2,732050807568878 & $0,00 \mathrm{e}+00$ & $0,00 \mathrm{e}+00$ & 921 & 463 & 0,04 \\
\hline & 5 & 3,020725942163691 & 3,020725942163691 & $0,00 \mathrm{e}+00$ & $0,00 \mathrm{e}+00$ & 9.112 & 3.693 & 0,36 \\
\hline & 6 & 3,232050807568878 & 3,232050807568878 & $0,00 \mathrm{e}+00$ & $0,00 \mathrm{e}+00$ & 34.669 & 13.086 & 1,25 \\
\hline & 7 & 3,443375672974062 & 5672974062 & $0,00 \mathrm{e}+00$ & $0,00 \mathrm{e}+00$ & 195.958 & 96.956 & 7,26 \\
\hline & 8 & 3,598076211353316 & 3,59807 & $0,00 \mathrm{e}+00$ & $0,00 \mathrm{e}+00$ & $1.242,459$ & 600.426 & 51,12 \\
\hline & 9 & 3,809252482546021 & 3,809401076758503 & $3,90 \mathrm{e}-05$ & $1,49 \mathrm{e}-04$ & $22.397,280$ & $8.929,210$ & 872,38 \\
\hline & 10 & 4,020335713626388 & 4,020725942163690 & $9,71 \mathrm{e}-05$ & $3,90 \mathrm{e}-04$ & $191.614,100$ & $81.788,232$ & $10.443,75$ \\
\hline \multirow{10}{*}{ 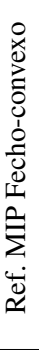 } & 1 & 1,577350269189625 & 1,577350269189625 & $0,00 \mathrm{e}+00$ & $0,00 \mathrm{e}+00$ & 0 & 0 & 0,01 \\
\hline & 2 & 38379251 & 2,154 & $0,00 \mathrm{e}+00$ & $0,00 \mathrm{e}+00$ & 59 & 0 & 0,00 \\
\hline & 3 & 2,443375672974065 & 2,4433756 & $0,00 \mathrm{e}+00$ & $0,00 \mathrm{e}+00$ & 733 & 76 & 0,05 \\
\hline & 4 & 2,732050807568877 & 2,732050807568877 & $0,00 \mathrm{e}+00$ & $0,00 \mathrm{e}+00$ & 4.883 & 686 & 0,18 \\
\hline & 5 & 3,020725942163687 & 3,020725942163687 & $0,00 \mathrm{e}+00$ & $0,00 \mathrm{e}+00$ & 69.208 & 6.219 & 2,81 \\
\hline & 6 & 3,232050807568884 & 3,232050807568884 & $0,00 \mathrm{e}+00$ & $0,00 \mathrm{e}+00$ & 357.205 & 28.210 & 14,42 \\
\hline & 7 & 3,443375672973989 & 3,443375672973989 & $0,00 \mathrm{e}+00$ & $0,00 \mathrm{e}+00$ & $1.948,856$ & 184.532 & 83,96 \\
\hline & 8 & 3,597775882542360 & 3,598076211353317 & $8,35 \mathrm{e}-05$ & $3,00 \mathrm{e}-04$ & $12.018,350$ & $1.126,677$ & 581,89 \\
\hline & 9 & 3,886402767942472 & 3,886751345948120 & $8,97 \mathrm{e}-05$ & $3,49 \mathrm{e}-04$ & - & - & $>21.600,00$ \\
\hline & 10 & 3,892320595579104 & 4,154700528069849 & $6,32 \mathrm{e}-02$ & $2,62 \mathrm{e}-01$ & - & - & $>21.600,00$ \\
\hline
\end{tabular}

Tabela 2.2: Instâncias do modelo $L G D P \mathcal{T}_{L G D P}^{k,[\Omega, h, w]}$ e suas reformulações $M I P \mathcal{T}_{B M}^{k,[\Omega, h, w]}$ e $\mathcal{T}_{C H}^{k,[\Omega, h, w]}$ com quantidade crescente de itens $N \in\{1,2, \ldots\}$. A restrição de eliminação de simetrias (2.8) foi considerada ao resolvermos $k+1$ instâncias do modelo (2.7,2.10), fixando os valore de $r_{i}$ de acordo com (2.14) para $p=0, \ldots, k$.

A Tabela 2.4 mostra os resultados. Notamos que substituir as restrições de não sobreposição (2.2) pelas de não sobreposição mais as restrições de eliminação de simetrias (2.11) é muito mais eficiente do que adicionar as restrições de eliminação de simetrias $(2.8,2.10)$ (veja as Tabelas 2.2 e 2.3), já que instâncias com valores maiores de $N$ foram resolvidas no primeiro caso. A Figura 2.4 mostra uma representação gráfica das soluções descritas na Tabela 2.4 para $\mathcal{U}_{B M}^{k,[\Omega, h, w]}$ variando a quantidade de retângulos de 1 até 15.

\subsubsection{Empacotando a maior quantidade de retângulos possível}

Nesta subseção lidaremos com o problema de, dado um triângulo equilátero com dimensões fixas, encontrar a maior quantidade $N$ de itens retangulares idênticos que podem ser empacotados nele (lembre-se que esses itens retangulares possuem altura $h=1$ e comprimento $w=0,5$ ).

Como detalhado na Seção 2.2, a estratégia para empacotar a maior quantidade de itens retangulares possível consiste em resolver $\mathcal{P}_{L G D P}^{k,[\Omega, h, w]}$ (o modelo de viabilidade LGDP (2.1-2.3)), ou qualquer um de suas reformulações MIP $\mathcal{P}_{B M}^{k,[\Omega, h, w]}$ e $\mathcal{P}_{C H}^{k,[\Omega, h, w]}$, para valores crescentes de $k$ até que um problema com $k^{\prime}$ itens seja provado inviável. Na prática, um limite de tempo é imposto para esta estratégia. Se dentro do limite de tempo a instância inviável é atingida e resolvida (ou seja, provada inviável), então está claro que a solução ótima é a solução da instância com $N=k^{\prime}-1$ itens. Por outro lado, se a estratégia é interrompida por conta do limite de tempo, temos duas possi- 


\begin{tabular}{|c|c|c|c|c|c|c|c|c|}
\hline \multicolumn{6}{|c|}{ Dados da instância } & \multicolumn{3}{|c|}{ Medidas de esforço } \\
\hline & \multirow{2}{*}{$N$ fixo } & \multicolumn{2}{|c|}{ Lado do Triângulo } & \multicolumn{2}{|c|}{ GAP MIP } & \multirow{2}{*}{ MIP } & \multirow{2}{*}{ Nós $B \& B$} & \multirow{2}{*}{ Tempo de CPU } \\
\hline & & Melhor limitante inferior & Melhor solução viável & Relativo & Absoluto & & & \\
\hline \multirow{10}{*}{ 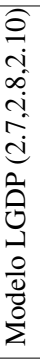 } & 1 & 1,577350269189626 & 1,577350269189626 & $0,00 \mathrm{e}+00$ & $0,00 \mathrm{e}+00$ & 0 & 0 & 0,00 \\
\hline & 2 & 2,154700538379252 & 2,154700538379252 & $0,00 \mathrm{e}+00$ & $0,00 \mathrm{e}+00$ & 5 & 0 & 0,00 \\
\hline & 3 & 2,443375672974065 & 2,443375672974065 & $0,00 \mathrm{e}+00$ & $0,00 \mathrm{e}+00$ & 148 & 37 & 0,02 \\
\hline & 4 & 2,732050807568881 & 2,732050807568881 & $0,00 \mathrm{e}+00$ & $0,00 e+00$ & 781 & 195 & 0,03 \\
\hline & 5 & 3,020725942163691 & 3,020725942163691 & $0,00 \mathrm{e}+00$ & $0,00 \mathrm{e}+00$ & 9.173 & 1.574 & 0,43 \\
\hline & 6 & 3,232050807568879 & 3,232050807568879 & $0,00 \mathrm{e}+00$ & $0,00 \mathrm{e}+00$ & 29.822 & 5.752 & 1,48 \\
\hline & 7 & 3,443375672974064 & 3,443375672974064 & $0,00 \mathrm{e}+00$ & $0,00 \mathrm{e}+00$ & 138.598 & 22.211 & 6,93 \\
\hline & 8 & 3,597794231919681 & 3,598076211353336 & $7,84 \mathrm{e}-05$ & $2,82 \mathrm{e}-04$ & 536.140 & 83.428 & 29,21 \\
\hline & 9 & 3,809064591545733 & 3,809401076758504 & $8,83 e-05$ & $3,36 e-04$ & $8.346,530$ & $1.109,399$ & 488,32 \\
\hline & 10 & 4,020338430165712 & 4,020725942163692 & $9,64 \mathrm{e}-05$ & $3,88 \mathrm{e}-04$ & $71.062,703$ & $10.825,791$ & $5.238,60$ \\
\hline \multirow{10}{*}{ 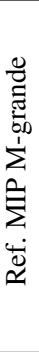 } & 1 & 1,577350269189626 & 1,577350269189626 & $0,00 \mathrm{e}+00$ & $0,00 \mathrm{e}+00$ & 0 & 0 & 0,00 \\
\hline & 2 & 2,154700538379251 & 2,154700538379251 & $0,00 \mathrm{e}+00$ & $0,00 \mathrm{e}+00$ & 23 & 5 & 0,00 \\
\hline & 3 & 2,443375672974064 & 2,443375672974064 & $0,00 \mathrm{e}+00$ & $0,00 e+00$ & 119 & 54 & 0,01 \\
\hline & 4 & 2,732050807568878 & 2,732050807568878 & $0,00 \mathrm{e}+00$ & $0,00 \mathrm{e}+00$ & 754 & 315 & 0,04 \\
\hline & 5 & 3,020725942163691 & 3,020725942163690 & $4,41 \mathrm{e}-16$ & $1,33 \mathrm{e}-15$ & 5.564 & 2.231 & 0,23 \\
\hline & 6 & 3,232050807568877 & 3,232050807568877 & $0,00 \mathrm{e}+00$ & $0,00 \mathrm{e}+00$ & 32.150 & 128.80 & 1,22 \\
\hline & 7 & 3,443375672974065 & 3,443375672974065 & $0,00 \mathrm{e}+00$ & $0,00 \mathrm{e}+00$ & 131.457 & 62.731 & 5,99 \\
\hline & 8 & 3,598076211353317 & 3,598076211353317 & $0,00 \mathrm{e}+00$ & $0,00 \mathrm{e}+00$ & 852.407 & 391.708 & 42,24 \\
\hline & 9 & 3,809031272878700 & 3,809401076758504 & $9,71 \mathrm{e}-05$ & $3,70 e-04$ & $37.824,870$ & $12.474,040$ & $2.935,49$ \\
\hline & 10 & 4,020324767277819 & 4,020725939008989 & $9,98 \mathrm{e}-05$ & $4,01 \mathrm{e}-04$ & $165.798,740$ & $58.305,517$ & $15.722,49$ \\
\hline \multirow{10}{*}{ 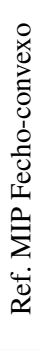 } & 1 & 1,577350269189626 & 1,577350269189626 & $0,00 \mathrm{e}+00$ & $0,00 \mathrm{e}+00$ & 0 & 0 & 0,00 \\
\hline & 2 & 2,154700534174552 & 2,154700534174552 & $0,00 \mathrm{e}+00$ & $0,00 \mathrm{e}+00$ & 63 & 15 & 0,01 \\
\hline & 3 & 2,443374238386405 & 2,443375672363309 & $5,87 \mathrm{e}-07$ & $1,43 \mathrm{e}-06$ & 1.282 & 166 & 0,07 \\
\hline & 4 & 2,732045702433151 & 2,732050806969059 & $1,87 \mathrm{e}-06$ & $5,10 \mathrm{e}-06$ & 12.153 & 1.227 & 0,43 \\
\hline & 5 & 3,020505520742559 & 3,020725922875648 & $7,30 \mathrm{e}-05$ & $2,20 \mathrm{e}-04$ & 157.030 & 13.592 & 5,60 \\
\hline & 6 & 3,231730502831837 & 3,232050797184711 & $9,91 \mathrm{e}-05$ & $3,20 \mathrm{e}-04$ & $1.936,538$ & 139.754 & 76,34 \\
\hline & 7 & 3,443031366455914 & 3,443375648663827 & $1,00 \mathrm{e}-04$ & $3,44 e-04$ & $25.129,693$ & $1.802,254$ & $1.222,66$ \\
\hline & 8 & 3,597716312942202 & 3,598076064926102 & $1,00 \mathrm{e}-04$ & $3,60 e-04$ & - & - & $>21.600,00$ \\
\hline & 9 & 3,207584396989211 & 3,886749550355905 & $1,75 \mathrm{e}-01$ & $6,79 \mathrm{e}-01$ & - & - & $>21.600,00$ \\
\hline & 10 & 3,105662430987886 & 4,020725345200177 & $2,28 \mathrm{e}-01$ & $9,15 \mathrm{e}-01$ & - & - & $>21.600,00$ \\
\hline
\end{tabular}

Tabela 2.3: Instâncias do modelo LGDP $\mathcal{T}_{L G D P}^{k,[\Omega, h, w]}$ e suas reformulações $M I P \mathcal{T}_{B M}^{k,[\Omega, h, w]}$ e $\mathcal{T}_{C H}^{k,[\Omega, h, w]}$ com quantidades crescentes de itens $(N \in\{1,2, \ldots\})$. A restrição de eliminação de simetria (2.8) foi considerada explicitamente no modelo.

bilidades. Considerando que o último problema resolvido possui, digamos, $k^{\prime \prime}$ itens (supondo que $k^{\prime \prime} \geq 1$ ): (a) $k^{\prime \prime}$ representa uma solução subótima e um empacotamento com pelo menos $k^{\prime \prime}+1$ itens exsite, mas não houve tempo de resolvê-la; ou (b) $k^{\prime \prime}$ é a quantidade máxima de itens que pode ser empacotada, mas não houve tempo suficiente para provar que a instância com $k^{\prime \prime}+1$ é inviável.

No primeiro conjunto de experimentos desta subseção, consideramos dez instâncias diferentes de $\mathcal{P}_{L G D P}^{k,[\Omega, h, w]}$ (modelo de viabilidade LGDP (2.1-2.3)), $\mathcal{P}_{B M}^{k,[\Omega, h, w]}$ (reformulação M-grande), e $\mathcal{P}_{C H}^{k,[\Omega, h, w]}$ (reformulação do fecho-convexo). Nessas dez instâncias, o lado do triângulo foi fixado de acordo com as melhores soluções viáveis apresentadas nas Tabelas 2.1-2.4. Dessa forma, um limitante inferior no número de itens que podem ser empacotados em cada triângulo é conhecido. A Tabela 2.5 mostra alguns dados relacionados com a solução destes problemas. Nessa tabela, o tempo de CPU é apresentado em detalhe. A primeira coluna, chamada "Total" representa o tempo de CPU total usado para resolver o problema ou o tempo transcorrido até o limite de tempo ser atingido. As outras três colunas indicam: (i) o tempo de CPU utilizado para resolver instâncias com $1, \ldots, N-1$ itens; (ii) o tempo de CPU usado para resolver a maior instância viável com $N$ itens; e (iii) o tempo de CPU utilizado para resolver a instância inviável, com $N+1$ itens. Perceba que somente as sete primeiras instâncias foram resolvidas dentro do limite de seis horas. Nos três casos restantes, a solução ótima foi de fato atingida, mas o tempo limite foi alcançado antes da inviabilidade ter sido provada. Note que a quantidade de tempo utilizada para encontrar a solução ótima é sempre muito pequena e que a maior parte do tempo computacional é usada para provar 


\begin{tabular}{|c|c|c|c|c|c|c|c|c|}
\hline \multicolumn{6}{|c|}{ Dados da instância } & \multicolumn{3}{|c|}{ Medidas de esforço } \\
\hline & \multirow[b]{2}{*}{$N$ fixo } & \multicolumn{2}{|c|}{ Lado do Triângulo } & \multicolumn{2}{|c|}{ GAP MIP } & \multirow{2}{*}{ MIP } & \multirow{2}{*}{ Nós B\&B } & \multirow{2}{*}{ Tempo de CPU } \\
\hline & & Melhor limitante inferior & Melhor solução viável & Relativo & Absoluto & & & \\
\hline \multirow{16}{*}{ 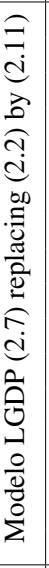 } & 1 & 1,577350269189626 & 1,577350269189626 & $0,00 \mathrm{e}+00$ & $0,00 \mathrm{e}+00$ & 0 & 0 & 0,00 \\
\hline & 2 & 2,154700538379251 & 2,154700538379251 & $0,00 \mathrm{e}+00$ & $0,00 \mathrm{e}+00$ & 5 & 0 & 0,00 \\
\hline & 3 & 2,443375672974064 & 2,443375672974064 & $0,00 \mathrm{e}+00$ & $0,00 \mathrm{e}+00$ & 46 & 7 & 0,02 \\
\hline & 4 & 2,732050807568879 & 2,732050807568879 & $0,00 \mathrm{e}+00$ & $0,00 \mathrm{e}+00$ & 94 & 10 & 0,00 \\
\hline & 5 & 3,020725942163691 & 3,020725942163691 & $0,00 \mathrm{e}+00$ & $0,00 \mathrm{e}+00$ & 286 & 61 & 0,02 \\
\hline & 6 & 3,232050807568878 & 3,232050807568878 & $0,00 \mathrm{e}+00$ & $0,00 \mathrm{e}+00$ & 738 & 173 & 0,08 \\
\hline & 7 & 3,443375672974065 & 3,443375672974065 & $0,00 \mathrm{e}+00$ & $0,00 \mathrm{e}+00$ & 3.193 & 715 & 0,15 \\
\hline & 8 & 3,598076211353317 & 3,598076211353317 & $0,00 \mathrm{e}+00$ & $0,00 \mathrm{e}+00$ & 16.115 & 2.847 & 0,75 \\
\hline & 9 & 3,809401076758504 & 3,809401076758504 & $0,00 \mathrm{e}+00$ & $0,00 \mathrm{e}+00$ & 71.257 & 11.960 & 3,46 \\
\hline & 10 & 4,020509285973630 & 4,020725942163690 & $5,39 \mathrm{e}-05$ & $2,17 \mathrm{e}-04$ & 203.721 & 41.891 & 12,03 \\
\hline & 11 & 4,175304993828159 & 4,175426480542942 & $2,91 \mathrm{e}-05$ & $1,21 \mathrm{e}-04$ & 927.056 & 110.081 & 43,67 \\
\hline & 12 & 64750972 & 29 & $8,57 \mathrm{e}-05$ & $3,76 \mathrm{e}-04$ & $3.244,798$ & 824 & 156,11 \\
\hline & 13 & 57176 & 61 & -05 & 4,42 & 0,447 & 2.48 & 6,96 \\
\hline & 14 & 023785 & 18 & $9,79 \mathrm{e}-05$ & 4,50 & $84.549,515$ & $12.186,953$ & 9,75 \\
\hline & 15 & 3416399242 & 415 & $7,01 \mathrm{e}-02$ & $3,33 \mathrm{e}-01$ & - & - & $>21.600,00$ \\
\hline & 16 & 581659 & 53 & $1,22 \mathrm{e}-01$ & $6,07 \mathrm{e}-01$ & - & - & 00,00 \\
\hline \multirow{16}{*}{ 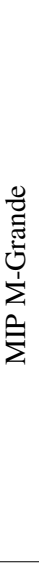 } & 1 & 9189626 & & +00 & $\overline{0,0}$ & 0 & 0 & 0,00 \\
\hline & 2 & 79251 & 51 & +00 & 0,0 & 14 & 0 & 0,00 \\
\hline & 3 & 2974064 & 64 & +00 & 0,0 & 49 & 7 & 0,01 \\
\hline & 4 & 568878 & & +00 & 0,0 & 105 & 13 & 0,00 \\
\hline & 5 & 2163691 & 91 & +00 & 0,0 & 416 & 91 & 0,02 \\
\hline & 6 & 3,232050807568878 & 3,232050807568878 & $0,00 \mathrm{e}+00$ & $0,00 \mathrm{e}+00$ & 1.619 & 373 & 0,06 \\
\hline & 7 & 3,443216646206837 & 3,443375672974065 & $4,62 \mathrm{e}-05$ & $1,59 \mathrm{e}-04$ & 7.003 & 1.462 & 0,24 \\
\hline & 8 & 3,598076211353316 & 3,598076211353316 & $0,00 \mathrm{e}+00$ & $0,00 \mathrm{e}+00$ & 14.739 & 2.622 & 0,54 \\
\hline & 9 & 3,809401076758503 & 3,809401076758503 & $0,00 \mathrm{e}+00$ & $0,00 \mathrm{e}+00$ & 51.510 & 5.636 & 1,54 \\
\hline & 10 & 4,020725942163691 & 4,020725942163691 & $0,00 \mathrm{e}+00$ & $0,00 \mathrm{e}+00$ & 486.468 & 77.816 & 16,25 \\
\hline & 11 & 4,175088319554543 & 4,175426480542939 & $8,10 \mathrm{e}-05$ & $3,38 \mathrm{e}-04$ & 857.439 & 103.487 & 32,44 \\
\hline & 12 & 4,386326500032685 & 4,386751345948128 & $9,68 \mathrm{e}-05$ & $4,25 \mathrm{e}-04$ & $6.235,708$ & 695.202 & 244,93 \\
\hline & 13 & 4,463655413028338 & 4,464101615137753 & $1,00 \mathrm{e}-04$ & $4,46 \mathrm{e}-04$ & $27.231,140$ & $3.427,145$ & $1.327,47$ \\
\hline & 14 & 4,597616595058138 & 4,598076211353316 & $1,00 \mathrm{e}-04$ & $4,60 \mathrm{e}-04$ & $80.702,664$ & $5.283,372$ & $3.402,18$ \\
\hline & 15 & 4,752303388377661 & 4,752776749732567 & $9,96 \mathrm{e}-05$ & $4,73 e-04$ & $165.176,634$ & $26.793,091$ & $9.019,73$ \\
\hline & 16 & 513204312 & 4,964101607673653 & $1,30 \mathrm{e}-01$ & $6,46 \mathrm{e}-01$ & - & - & $>21.600,00$ \\
\hline \multirow{16}{*}{ 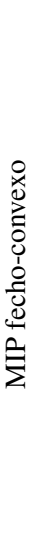 } & 1 & 1,577350269189626 & 577350269189626 & $0,00 \mathrm{e}+00$ & $0,00 \mathrm{e}+00$ & 0 & 0 & 0,00 \\
\hline & 2 & 2,154700538070694 & 538381463 & $1,44 \mathrm{e}-10$ & $3,11 \mathrm{e}-10$ & 40 & 7 & 0,02 \\
\hline & 3 & 572645347 & 645347 & $0,00 \mathrm{e}+00$ & $0,00 \mathrm{e}+00$ & 201 & 33 & 0,01 \\
\hline & 4 & 483621 & 3621 & $0,00 \mathrm{e}+00$ & $0,00 \mathrm{e}+00$ & 1.019 & 139 & 0,07 \\
\hline & 5 & 3,020725579211940 & 942196470 & $1,20 \mathrm{e}-07$ & $3,63 \mathrm{e}-07$ & 3.408 & 506 & 0,26 \\
\hline & 6 & 3,231850013972345 & 232050807511775 & $6,21 \mathrm{e}-05$ & $2,01 \mathrm{e}-04$ & 12.039 & 1.458 & 0,61 \\
\hline & 7 & 3,443202829554564 & 443375666133137 & $5,02 \mathrm{e}-05$ & $1,73 \mathrm{e}-04$ & 46.810 & 5.859 & 3,18 \\
\hline & 8 & 3,597886815976836 & 598076209758018 & $5,26 \mathrm{e}-05$ & $1,89 \mathrm{e}-04$ & 123.158 & 13.064 & 8,20 \\
\hline & 9 & 3,809272419509341 & 809401078416926 & $3,38 \mathrm{e}-05$ & $1,29 \mathrm{e}-04$ & 662.582 & 73.940 & 51,17 \\
\hline & 10 & 4,020327421884938 & 20725794811397 & $9,91 \mathrm{e}-05$ & $3,98 \mathrm{e}-04$ & $4.930,428$ & 54.196 & 445,95 \\
\hline & 11 & 4,175027994557123 & 4,175426437952257 & $9,54 \mathrm{e}-05$ & $3,98 \mathrm{e}-04$ & $18.041,399$ & $1.720,300$ & $1.797,97$ \\
\hline & 12 & 4,366025218173216 & 4,386751328238087 & $4,72 \mathrm{e}-03$ & $2,07 \mathrm{e}-02$ & - & - & $>21.600,00$ \\
\hline & 13 & 4,166912549242940 & 4,464101448383035 & $6,66 \mathrm{e}-02$ & $2,97 \mathrm{e}-01$ & - & - & $>21.600,00$ \\
\hline & 14 & 3,963950046260996 & 4,598075130802665 & $1,38 \mathrm{e}-01$ & $6,34 \mathrm{e}-01$ & - & - & $>21.600,00$ \\
\hline & 15 & 3,787499813498732 & 4,752776715788993 & $2,03 \mathrm{e}-01$ & $9,65 \mathrm{e}-01$ & - & - & $>21.600,00$ \\
\hline & 16 & 3,845092857113551 & 4,964101312861107 & $2,25 \mathrm{e}-01$ & $1,12 \mathrm{e}+00$ & - & - & $>21.600,00$ \\
\hline
\end{tabular}

Tabela 2.4: Instâncias do modelo $L G D P \mathcal{U}_{L G D P}^{k,[\Omega, h, w]}$ e suas reformulações $\mathcal{U}_{B M}^{k,[\Omega, h, w]}$ e $\mathcal{U}_{C H}^{k,[\Omega, h, w]}$ para um número crescente $N \in\{1,2, \ldots\}$.

que a solução encontrada é de fato ótima. Esta observação justifica o aumento sequencial de $N$ em contraste com uma abordagem mais intuitiva como a bissecção.

As Tabelas 2.6 e 2.7 mostram as soluções obtidas ao se resolver as mesmas dez instâncias ( para os mesmos lados fixos dos triângulos já utilizadas nos experimentos da Tabela 2.5) considerando agora os problemas $\mathcal{Q}_{L G D P}^{k,[\Omega, h, w]}$ (Modelo LGDP (2.1-2.3,2.8,2.10)), $\mathcal{Q}_{B M}^{k,[\Omega, h, w]}$ (reformulação Mgrande), e $\mathcal{Q}_{C H}^{k,[\Omega, h, w]}$ (reformulação do fecho-convexo); e $\mathcal{R}_{L G D P}^{k,[\Omega, h, w]}$ (Modelo LGDP $(2.1,2.11,2.8)$ ), $\mathcal{R}_{B M}^{k,[\Omega, h, w]}$ (reformulação M-grande), e $\mathcal{R}_{C H}^{k,[\Omega, h, w]}$ (reformulação do fecho-convexo), respectivamente. A Figura 2.5 ilustra a representação gráfica das soluções descritas na Tabela 2.7 para $\mathcal{R}_{B M}^{k,[\Omega, h, w]}$ com 


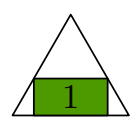

$N=1$

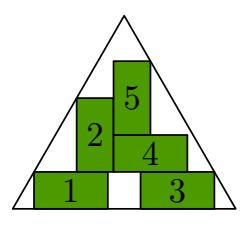

$N=5$

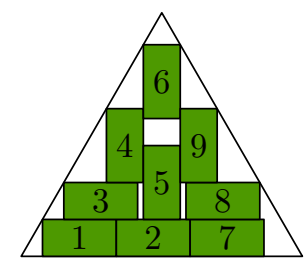

$N=9$

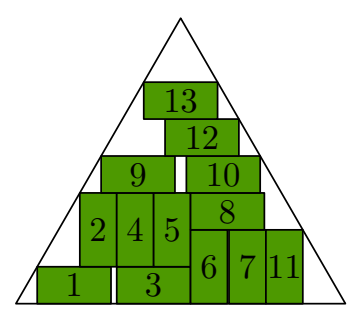

$N=13$

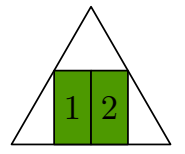

$N=2$

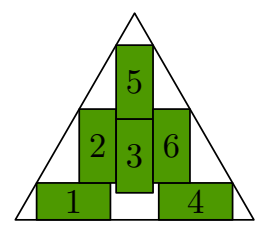

$N=6$

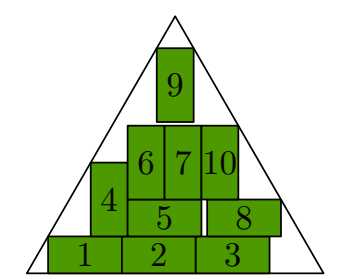

$N=10$

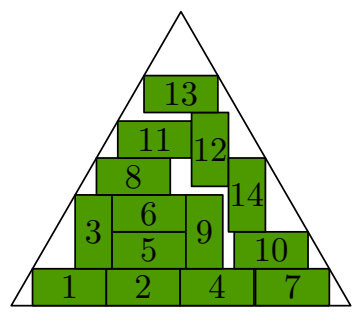

$N=14$

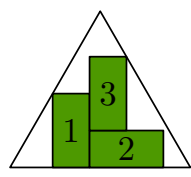

$N=3$

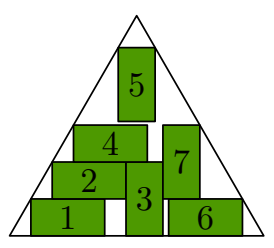

$N=7$

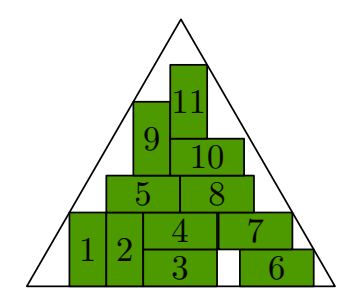

$N=11$

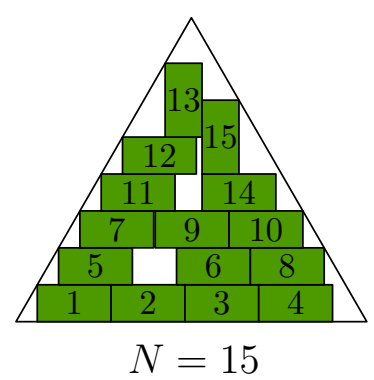

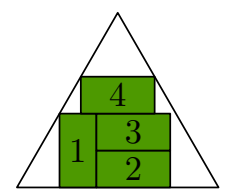

$N=4$

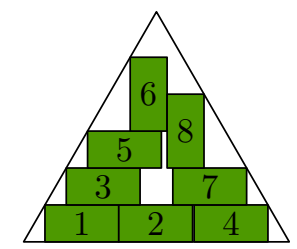

$N=8$

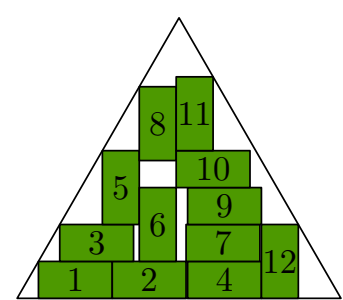

$N=12$

Figura 2.4: Representação gráfica da solução para o problema de minimizar o lado de um triângulo equilátero que contém $N \in\{1,2, \ldots, 15\}$ retângulos idênticos, com altura $h=1$ e comprimento $w=0,5$. As figuras correspondem às soluções das instâncias de $\mathcal{U}_{B M}^{k,[\Omega, h, w]}$ descritas na Tabela 2.4. Note que elas satisfazem as restrições (2.11) ou suas reformulações (2.12) e (2.13).

diferentes quantidades de retângulos, de 1 a 14. Nota-se que as restrições de eliminação de simetrias ajudam muito a reduzir drasticamente o esforço necessário para resolver as instâncias. De fato, com a ajuda dessas restrições, as soluções ótimas de dez instâncias foram encontradas e provadas ótimas e até instâncias maiores puderam ser resolvidas até a otimalidade. A comparação entre os tempos mostrados nas Tabelas 2.5-2.7 é bastante elucidativa. Quando uma instância com uma quantidade fixa $k$ de itens é viável, parece que eliminar soluções simétricas com a ajuda das restrições de eliminação de simetrias não é vantajoso. Observe que os tempos de CPU para resolver as instâncias com $N$ itens na Tabela 2.5 (que corresponde aos experimentos com os modelos sem as restrições de eliminação de simetrias) são menores que aqueles mostrados nas Tabelas 2.6 e 2.7, que considera as restrições de eliminação de simetrias. Uma explicação para esse fenômeno parece ser bem simples. Se a instância de um problema de viabilidade é viável, quanto maior a quantidade de soluções viáveis (simétricas ou não), mais rápido o solver encontrará uma e declarará sucesso. A situação oposta ocorre quando comparamos os tempos de CPU usados para resolver as instâncias inviáveis com $N+1$ itens das Tabelas 2.5-2.7. Como as instâncias são inviáveis, toda a árvore de busca deve ser visitada para provar a inviabilidade. Neste caso, eliminar soluções simétricas faz a árvore de busca ser menor, transformando a tarefa de provar a inviabilidade em um trabalho menos difícil. Fazendo um balanço das duas situações, considerar as restrições de eliminação de 


\begin{tabular}{|c|c|c|c|c|c|c|c|c|}
\hline \multicolumn{3}{|c|}{ Dados da instância } & \multicolumn{6}{|c|}{ Medidas de esforço } \\
\hline & \multirow{2}{*}{ Lado do triângulo } & \multirow{2}{*}{ Melhor $N$} & \multirow{2}{*}{ MIP } & \multirow{2}{*}{ Nós $B \& B$} & \multicolumn{4}{|c|}{ Tempo de CPU } \\
\hline & & & & & Total & 1 to $N-1$ & $N$ & $N+1$ \\
\hline \multirow{10}{*}{ 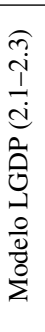 } & 1,577350269189625 & 1 & 0 & 0 & 0,01 & - & 0,00 & 0,01 \\
\hline & 2,154700529884166 & 2 & 112 & 15 & 0,01 & 0,00 & 0,00 & 0,01 \\
\hline & 2,443375667541825 & 3 & 444 & 149 & 0,04 & 0,01 & 0,00 & 0,03 \\
\hline & 2,732050717264398 & 4 & 17.882 & 3.270 & 0,63 & 0,01 & 0,00 & 0,62 \\
\hline & 3,020725894730525 & 5 & 337.480 & 54.092 & 10,31 & 0,02 & 0,00 & 10,29 \\
\hline & 3,232050397095424 & 6 & $1.702,397$ & 288.180 & 64,57 & 0,02 & 0,02 & 64,53 \\
\hline & 3,443373062238137 & 7 & $310.606,602$ & $41.339,182$ & $20.824,26$ & 0,04 & 0,08 & $20.824,14$ \\
\hline & 3,598076064926102 & 8 & - & - & $>21.600,00$ & 0,06 & 0,03 & - \\
\hline & 3,809401068449104 & 9 & - & - & $>21.600,00$ & 0,08 & 4,57 & - \\
\hline & 4,020724784525934 & 10 & - & - & $>21.600,00$ & 0,09 & 0,44 & - \\
\hline \multirow{10}{*}{ 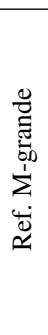 } & 1,577350269189625 & 1 & 0 & 0 & 0,00 & - & 0,00 & 0,00 \\
\hline & 2,154700529884166 & 2 & 61 & 23 & 0,01 & 0,00 & 0,00 & 0,01 \\
\hline & 2,443375667541825 & 3 & 484 & 213 & 0,02 & 0,00 & 0,00 & 0,02 \\
\hline & 2,732050717264398 & 4 & 10.844 & 4.847 & 0,31 & 0,00 & 0,01 & 0,30 \\
\hline & 3,020725894730525 & 5 & 340.644 & 133.095 & 9,21 & 0,00 & 0,01 & 9,20 \\
\hline & 3,232050397095424 & 6 & $5.891,214$ & 436 & 193,02 & 0,01 & 0,01 & 193,00 \\
\hline & 3,443373062238137 & 7 & $346.797,622$ & $132.731,792$ & $12.484,64$ & 0,02 & 0,02 & $12.484,60$ \\
\hline & 3,598076064926102 & 8 & - & - & $>21.600,00$ & 0,04 & 0,36 & - \\
\hline & 3,809401068449104 & 9 & - & - & $>21.600,00$ & 0,05 & 0,57 & - \\
\hline & 4,020724784525934 & 10 & - & - & $>21.600,00$ & 0,05 & 0,22 & - \\
\hline \multirow{10}{*}{ 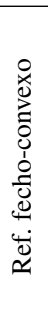 } & 1,577350269189625 & 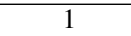 & 51 & 1 & 0,01 & - & 0,00 & 0,01 \\
\hline & 2,154700529884166 & 2 & 839 & 94 & 0,04 & 0,00 & 0,01 & 0,03 \\
\hline & 2,443375667541825 & 3 & 11.087 & 1.016 & 0,30 & 0,01 & 0,01 & 0,28 \\
\hline & 2,732050717264398 & 4 & 294.957 & 16.712 & 8,76 & 0,02 & 0,03 & 8,71 \\
\hline & 3,020725894730525 & 5 & $10.026,367$ & 632.425 & 301,85 & 0,03 & 0,13 & 301,69 \\
\hline & 3,232050397095424 & 6 & - & - & $>21.600,00$ & 0,07 & 1,53 & - \\
\hline & 3,443373062238137 & 7 & - & - & $>21.600,00$ & 0,13 & 2,77 & - \\
\hline & 3,598076064926102 & 8 & - & - & $>21.600,00$ & 1,31 & 5,91 & - \\
\hline & 3,809401068449104 & 9 & - & - & $>21.600,00$ & 0,60 & 871,80 & - \\
\hline & 4,020724784525934 & 10 & - & - & $>21.600,00$ & 0,93 & 221,04 & - \\
\hline
\end{tabular}

Tabela 2.5: Instâncias de $\mathcal{P}_{L G D P}^{k,[\Omega, h, w]}, \mathcal{P}_{B M}^{k,[\Omega, h, w]}$, e $\mathcal{P}_{C H}^{k,[\Omega, h, w]}$ fixando o lado do triângulo equilátero como a melhor solução viável ilustrada nas Tabelas 2.1-2.4.

simetrias parece vantajoso. Mais ainda, o melhor dos dois cenários poderia, talvez, ser aproveitado em uma abordagem heurística combinada para rapidamente encontrar uma solução que seja provavelmente ótima e então provar sua otimalidade.

\subsection{Conclusões}

Modelos LGDP e MIP para dois problemas de empacotamento foram apresentados e restrições de eliminação de simetrias foram introduzidas. As restrições de eliminação de simetrias ajudam, como esperado, com exceção do caso de verificação de viabilidade/inviabilidade quando o problema é viável. O maior benefício de incorporar restrições de eliminação de simetrias, em termos de reduzir o tempo de computação, é obtido no caso em que desejamos provar a otimalidade ou a inviabilidade de uma instância. As restrições de eliminação de simetrias que impõem uma préordem entre os itens e que enumeram os não rotacionados primeiro mostram-se úteis. Porém, as restrições de eliminação de simetrias que impõem a ordenação do tipo acima ou a direita parecem ser as mais úteis, já que simplificam as restrições de não sobreposição tornando todo o modelo mais fácil de ser resolvido (ao menos para os problemas considerados). Apesar do modelo LGDP original parecer mais simples para o solver no caso do problema de minimização do lado do triângulo, as reformulações MIP M-grande parecem mais adequadas no caso do problema de viabilidade. De qualquer forma, o modelo original LGDP e suas reformulações M-grande mostraram-se mais competitivas do que as reformulações MIP com o fecho-convexo. 


\begin{tabular}{|c|c|c|c|c|c|c|c|c|}
\hline \multicolumn{3}{|c|}{ Dados da instância } & \multicolumn{6}{|c|}{ Medidas de esforço } \\
\hline & \multirow{2}{*}{ Lado do triângulo } & \multirow{2}{*}{ Melhor $N$} & \multirow{2}{*}{ MIP } & \multirow{2}{*}{ Nós B\&B } & \multicolumn{4}{|c|}{ Tempo de CPU } \\
\hline & & & & & Total & 1 to $N-1$ & $N$ & $N+1$ \\
\hline \multirow{10}{*}{ 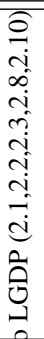 } & 1,577350269189625 & 1 & 0 & 0 & 0,01 & - & 0,00 & 0,01 \\
\hline & 2,154700529884166 & 2 & 0 & 0 & 0,01 & 0,00 & 0,00 & 0,01 \\
\hline & 2,443375667541825 & 3 & 0 & 0 & 0,03 & 0,00 & 0,01 & 0,02 \\
\hline & 2,732050717264398 & 4 & 272 & 49 & 0,06 & 0,00 & 0,01 & 0,05 \\
\hline & 3,020725894730525 & 5 & 10.604 & 1.575 & 0,39 & 0,01 & 0,07 & 0,31 \\
\hline & 3,232050397095424 & 6 & 53.965 & 7.822 & 2,20 & 0,02 & 0,83 & 1,35 \\
\hline & 3,443373062238137 & 7 & 151.158 & 21.720 & 5,14 & 0,05 & 0,86 & 4,50 \\
\hline & 3,598076064926102 & 8 & $3.486,287$ & 288.006 & 107,50 & 0,07 & 0,60 & 106,83 \\
\hline & 3,809401068449104 & 9 & $20.550,301$ & $1.819,760$ & 666,90 & 0,10 & 196,57 & 469,23 \\
\hline & 4,020724784525934 & 10 & $244.497,247$ & $18.368,674$ & $9.090,28$ & 3,11 & 442,74 & $8.644,43$ \\
\hline \multirow{10}{*}{ 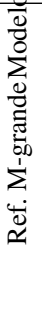 } & 1,577350269189625 & 1 & 0 & 0 & 0,00 & - & 0,00 & $\overline{0,00}$ \\
\hline & 2,154700529884166 & 2 & 0 & 0 & 0,00 & 0,00 & 0,00 & 0,00 \\
\hline & 2,443375667541825 & 3 & 222 & 64 & 0,04 & 0,00 & 0,01 & 0,03 \\
\hline & 2,732050717264398 & 4 & 1.374 & 626 & 0,08 & 0,00 & 0,01 & 0,08 \\
\hline & 3,020725894730525 & 5 & 20.295 & 6.363 & 0,66 & 0,00 & 0,11 & 0,55 \\
\hline & 3,232050397095424 & 6 & 71.477 & 22.973 & 2,26 & 0,02 & 0,78 & 1,46 \\
\hline & 3,443373062238137 & 7 & 348.952 & 104.069 & 10,32 & 0,02 & 1,06 & 9,24 \\
\hline & 3,598076064926102 & 8 & $3.198,793$ & 743.105 & 78,42 & 0,12 & 1,21 & 77,09 \\
\hline & 3,809401068449104 & 9 & $20.967,920$ & $4.103,489$ & 591,68 & 0,31 & 159,70 & 431,67 \\
\hline & 4,020724784525934 & 10 & $504.665,327$ & $92.696,919$ & $15.443,09$ & 0,14 & 213,67 & $15.229,28$ \\
\hline \multirow{10}{*}{ 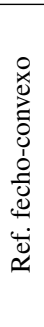 } & 1,577350269189625 & 1 & 0 & 0 & 0,01 & - & 0,00 & 0,01 \\
\hline & 2,154700529884166 & 2 & 141 & 0 & 0,02 & 0,00 & 0,00 & 0,02 \\
\hline & 2,443375667541825 & 3 & 865 & 16 & 0,08 & 0,01 & 0,01 & 0,06 \\
\hline & 2,732050717264398 & 4 & 12.495 & 712 & 0,47 & 0,00 & 0,00 & 0,47 \\
\hline & 3,020725894730525 & 5 & 189.844 & 6.809 & 7,46 & 0,01 & 0,58 & 6,87 \\
\hline & 3,232050397095424 & 6 & 888.823 & 31.810 & 34,58 & 0,04 & 11,32 & 23,22 \\
\hline & 3,443373062238137 & 7 & $9.305,812$ & 302.078 & 468,18 & 0,50 & 18,58 & 449,10 \\
\hline & 3,598076064926102 & 8 & $110.802,449$ & $2.211,931$ & $9.489,72$ & 0,91 & 27,68 & $9.461,13$ \\
\hline & 3,809401068449104 & 9 & - & - & $>21.600,00$ & 3,41 & - & - \\
\hline & 4,020724784525934 & 10 & - & - & $>21.600,00$ & 1,68 & - & - \\
\hline
\end{tabular}

Tabela 2.6: Instâncias de $\mathcal{Q}_{L G D P}^{k,[\Omega, h, w]}, \mathcal{Q}_{B M}^{k,[\Omega, h, w]}, e \mathcal{Q}_{C H}^{k,[\Omega, h, w]}$ fixando o lado dos triângulos equiláteros como as melhores soluções ilustradas nas Tabelas 2.1-2.4. 


\begin{tabular}{|c|c|c|c|c|c|c|c|c|}
\hline \multicolumn{3}{|c|}{ Dados da instância } & \multicolumn{6}{|c|}{ Medidas de esforço } \\
\hline & \multirow{2}{*}{ Lado do triângulo } & \multirow{2}{*}{ Melhor $N$} & \multirow{2}{*}{ MIP } & \multirow{2}{*}{ Nós B\&B } & \multicolumn{4}{|c|}{ Tempo de CPU } \\
\hline & & & & & Total & 1 to $N-1$ & $N$ & $N+1$ \\
\hline$\hat{m}$ & 1,577350269189625 & 1 & 0 & $\overline{0}$ & $\overline{0,00}$ & - & $\overline{0,00}$ & $\overline{0,00}$ \\
\hline กี & 2,154700529884166 & 2 & 3 & 0 & 0,00 & 0,00 & 0,00 & 0,00 \\
\hline $\bar{Z}$ & 2,443375667541825 & 3 & 9 & 0 & 0,01 & 0,00 & 0,01 & 0,00 \\
\hline 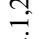 & 2,732050717264398 & 4 & 159 & 15 & 0,02 & 0,00 & 0,01 & 0,01 \\
\hline$\stackrel{0}{2}$ & 3,020725894730525 & 5 & 427 & 73 & 0,05 & 0,01 & 0,01 & 0,03 \\
\hline 官 & 3,232050397095424 & 6 & 634 & 157 & 0,07 & 0,01 & 0,02 & 0,04 \\
\hline త్త & 3,443373062238137 & 7 & 8.295 & 1.308 & 0,35 & 0,01 & 0,03 & 0,31 \\
\hline 으 & 3,598076064926102 & 8 & 14.626 & 2.915 & 0,60 & 0,04 & 0,03 & 0,53 \\
\hline \& & 3,809401068449104 & 9 & 43.669 & 9.693 & 1,71 & 0,04 & 0,11 & 1,56 \\
\hline$\Sigma$ & 4,020724784525934 & 10 & 611.197 & 64.388 & 20,51 & 0,06 & 0,03 & 20,42 \\
\hline & 4,175426437952257 & 11 & $2.190,175$ & 228.068 & 81,77 & 0,06 & 0,07 & 81,64 \\
\hline & 4,386751328238087 & 12 & $5.299,011$ & 987.112 & 212,68 & 0,08 & 0,14 & 212,46 \\
\hline & 4,464101448383035 & 13 & $34.795,370$ & $3.248,546$ & $1.540,14$ & 0,12 & 0,05 & $1.539,97$ \\
\hline & 4,598075130802665 & 14 & $205.848,392$ & $13.957,106$ & $10.299,73$ & 0,17 & 0,54 & $10.299,01$ \\
\hline & 4,752776715788993 & 15 & - & - & $>21.600,00$ & 0,22 & 0,07 & - \\
\hline & 1,577350269189625 & 1 & 0 & 0 & 0,01 & - & 0,00 & 0,01 \\
\hline & 2,154700529884166 & 2 & 19 & 0 & 0,00 & 0,00 & 0,00 & 0,00 \\
\hline 0 & 2,443375667541825 & 3 & 58 & 0 & 0,01 & 0,00 & 0,00 & 0,01 \\
\hline 芯 & 2,732050717264398 & 4 & 126 & 18 & 0,03 & 0,02 & 0,00 & 0,01 \\
\hline 50 & 3,020725894730525 & 5 & 447 & 70 & 0,03 & 0,01 & 0,00 & 0,02 \\
\hline$\sum$ & 3,232050397095424 & 6 & 805 & 175 & 0,04 & 0,01 & 0,00 & 0,04 \\
\hline ๘ं & 3,443373062238137 & 7 & 5.135 & 1.252 & 0,20 & 0,02 & 0,01 & 0,17 \\
\hline & 3,598076064926102 & 8 & 29.736 & 3.209 & 0,79 & 0,03 & 0,02 & 0,74 \\
\hline & 3,809401068449104 & 9 & 79.232 & 10.090 & 2,25 & 0,03 & 0,04 & 2,18 \\
\hline & 4,020724784525934 & 10 & 519.162 & 52.811 & 14,35 & 0,03 & 0,02 & 14,30 \\
\hline & 4,175426437952257 & 11 & $1.783,841$ & 157.266 & 50,15 & 0,05 & 0,04 & 50,06 \\
\hline & 4,386751328238087 & 12 & $10.102,510$ & $1.249,609$ & 358,40 & 0,04 & 0,11 & 358,25 \\
\hline & 4,464101448383035 & 13 & $34.466,909$ & $2.878,251$ & $1.276,59$ & 0,07 & 0,06 & $1.276,46$ \\
\hline & 4,598075130802665 & 14 & $122.262,705$ & $16.673,839$ & $3.636,51$ & 0,10 & 0,04 & $3.636,37$ \\
\hline & 4,752776715788993 & 15 & - & - & $>21.600,00$ & 0,13 & 0,03 & - \\
\hline & 1,577350269189625 & 1 & 27 & 0 & 0,01 & - & 0,00 & 0,01 \\
\hline & 2,154700529884166 & 2 & 118 & 12 & 0,02 & 0,00 & 0,00 & 0,02 \\
\hline$\sqrt[x]{0}$ & 2,443375667541825 & 3 & 401 & 44 & 0,06 & 0,01 & 0,01 & 0,04 \\
\hline $\overrightarrow{0}$ & 2,732050717264398 & 4 & 1.018 & 121 & 0,10 & 0,01 & 0,01 & 0,08 \\
\hline $\begin{array}{l}0 \\
0 \\
1\end{array}$ & 3,020725894730525 & 5 & 6.824 & 721 & 0,32 & 0,02 & 0,05 & 0,25 \\
\hline$\frac{\pi}{0}$ & 3,232050397095424 & 6 & 29.177 & 2.696 & 1,24 & 0,03 & 0,36 & 0,85 \\
\hline$\stackrel{\varpi}{\longleftarrow}$ & 3,443373062238137 & 7 & 119.510 & 8.104 & 5,46 & 0,06 & 0,39 & 5,01 \\
\hline$\stackrel{4}{2}$ & 3,598076064926102 & 8 & 396.333 & 21.332 & 19,86 & 0,11 & 0,85 & 18,90 \\
\hline & 3,809401068449104 & 9 & $2.136,966$ & 88.392 & 120,17 & 0,15 & 1,84 & 118,18 \\
\hline & 4,020724784525934 & 10 & $15.166,636$ & 482.411 & $1.046,81$ & 0,28 & 0,74 & $1.045,79$ \\
\hline & 4,175426437952257 & 11 & $46.304,928$ & $1.438,015$ & $4.317,87$ & 0,46 & 2,01 & $4.315,40$ \\
\hline & 4,386751328238087 & 12 & - & - & $>21.600,00$ & 0,71 & 17,03 & - \\
\hline & 4,464101448383035 & 13 & - & - & $>21.600,00$ & 0,80 & 9,98 & - \\
\hline & 4,598075130802665 & 14 & - & - & $>21.600,00$ & 1,36 & 48,78 & - \\
\hline & 4,752776715788993 & 15 & - & - & $>21.600,00$ & 6,80 & 37,14 & - \\
\hline
\end{tabular}

Tabela 2.7: Instâncias de $\mathcal{R}_{L G D P}^{k,[\Omega, h, w]}, \mathcal{R}_{B M}^{k,[\Omega, h, w]}$, e $\mathcal{R}_{C H}^{k,[\Omega, h, w]}$ definindo o lado do triângulo equilátero como as melhores soluções viáveis ilustradas nas Tabelas 2.1-2.4. 


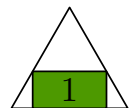

$N=1$

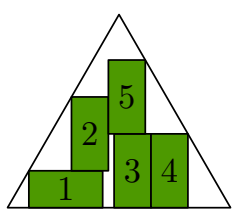

$N=5$

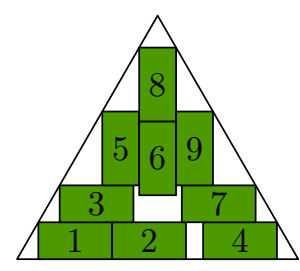

$N=9$

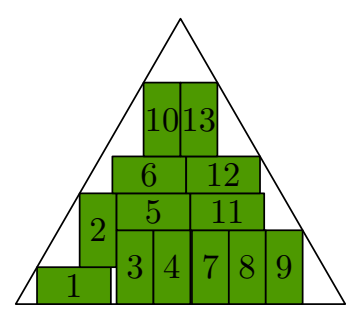

$N=13$

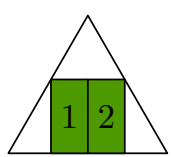

$N=2$

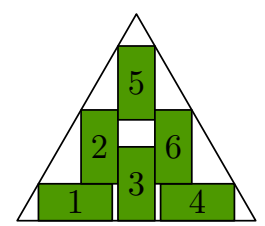

$N=6$

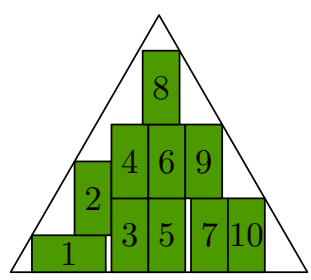

$N=10$

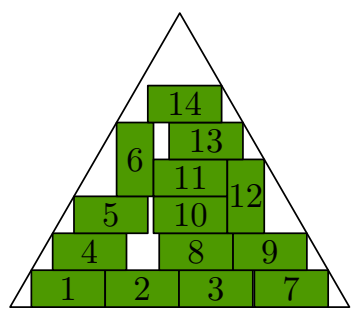

$N=14$

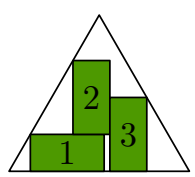

$N=3$

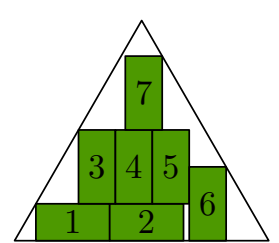

$N=7$

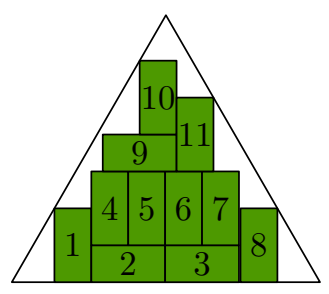

$\mathrm{N}=11$

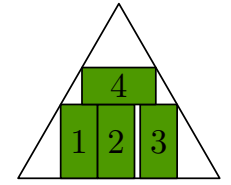

$N=4$

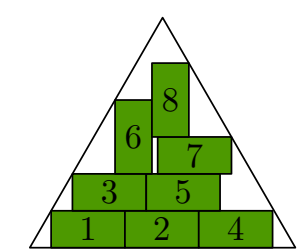

$N=8$

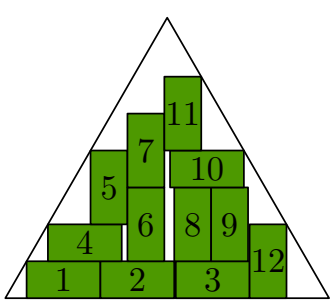

$\mathrm{N}=12$

Figura 2.5: Representação gráfica das soluções do problema de empacotar a maior quantidade possível de retângulos idênticos, com altura $h=1$ e comprimento $w=0,5$ em um triângulo equilátero de lado fixo. As figuras correspondem às soluções para instâncias de $\mathcal{R}_{B M}^{k,[\Omega, h, w]}$ descritas na Tabela 2.7. Note que elas satisfazem as restrições em (2.11) ou suas reformulações (2.12) e (2.13). 


\section{Capítulo 3}

\section{Problema de corte em dois estágios com sobras aproveitáveis}

Neste capítulo estudamos uma variação do problema de corte de itens retangulares com sobras aproveitáveis chamada de problema de corte em dois estágios com sobras aproveitáveis. Neste problema desejamos cortar uma quantidade finita de itens retangulares, obedecendo um padrão de corte em dois estágios, a partir de um conjunto finito de objetos retangulares de forma a minimizar o custo dos objetos utilizados e, dentre as diferentes formas de fazer isso, desejamos escolher a que maximiza o valor das sobras aproveitáveis. Um padrão de corte do tipo guilhotinado é aquele em que cada item deve ser obtido com uma sequência de cortes de uma extremidade a outra do objeto, paralelos às suas laterais. Um padrão de corte em dois estágios é um padrão de corte guilhotinado no qual se utiliza até dois conjuntos de cortes guilhotinados para obter cada item. No primeiro estágio, cortes guilhotinados são feitos paralelos ao comprimento (ou largura) do objeto, produzindo um conjunto de faixas longitudinais (ou transversais) e no segundo estágio são feitos cortes guilhotinados perpendiculares aos primeiros. Pode ainda ser necessário um terceiro conjunto de cortes guilhotinados (também chamados de trimming) para separar um item da sobra não aproveitável ou desperdício.

O problema de corte de itens bidimensionais em 2 estágios ou mais genericamente, em $k$ estágios, é um problema que ocorre em processos industriais de corte quando limita-se os padrões de corte a serem do tipo guilhotinado. No primeiro estágio são feitos cortes guilhotinados paralelos a um dos lados do objeto e no estágio seguinte são feitos cortes ortogonais aos anteriores e assim sucessivamente. Existem diversos trabalhos propondo diferentes métodos para esse problema, como [Gilmore65, Beasley85a, Morabito96]. Até onde pudemos verificar, não encontramos trabalhos considerando a questão de sobras aproveitáveis relacionada a problemas de corte em $k$ estágios. Consideramos como sobras aproveitáveis as sobras resultantes dos cortes longitudinais (exemplo ilustrado na Figura 3.1) que tiverem altura superior a um valor pré-determinado $d_{\min }$.

Na próxima seção apresentamos uma forma de modelar o problema de corte em dois estágios. Na Seção 3.2 introduzimos uma forma de incorporar as sobras ao modelo de corte em dois estágios, usando programação em dois níveis, e reformulamos o modelo como um MIP. Na Seção 3.3 expomos um segundo modelo para o problema de corte (sem sobras) que agrega os itens por tipo e mostramos como utilizá-lo na modelagem do problema de corte com sobras. Na Seção 3.4 apresentamos experimentos numéricos com os dois modelos apresentados para o problema de corte com sobras aproveitáveis e algumas conclusões. 


\subsection{Um modelo para o problema de corte em dois estágios}

Nesta seção apresentamos um modelo para o problema de corte em dois estágios. O problema de corte em dois estágios consiste em, dados $p$ objetos, obter um conjunto de padrões de corte em dois estágios para $n$ itens retangulares, de forma a minimizar o custo dos objetos utilizados. Consideramos que cada item possui altura $h_{i}$ e largura $w_{i}(i=1, \ldots, n)$ e cada objeto possui altura $H_{l}$, largura $W_{l}$ e custo $c_{l}(l=1, \ldots, p)$. Supomos, sem perda de generalidade, que $h_{1} \geq$ $h_{2} \geq \ldots \geq h_{n}$. Supomos também que todas as dimensões dos itens e objetos, bem como, os custos dos objetos, são inteiros. O modelo que apresentamos a seguir corresponde ao modelo M1 apresentado em [Lodi03], no qual os autores tratam do problema da mochila bidimensional em dois estágios (TDK - Two-Dimensional Knapsack), problema similar ao problema de corte em dois estágios. Embora tenhamos nos baseado fortemente no trabalho de [Lodi03], existem outros modelos para o problema da mochila bidimensional em dois estágios que poderiam ser utilizados, como os discutidos em [Morabito12].

A versão do modelo, que apresentamos a seguir, possui a função objetivo modificada e foi adaptada para incorporar mais de um objeto. No TDK temos um único objeto caracterizado por suas dimensões horizontal e vertical e uma lista de itens caracterizados por suas dimensões, lucro e limitante superior para a quantidade de itens a ser cortada. Neste problema deseja-se encontrar um padrão de corte que maximize a soma dos lucros dos itens a serem cortados. O caso do TDK com padrão de corte guilhotinado é conhecido como problema da mochila bidimensional em $d$ estágios, no qual a quantidade máxima de cortes guilhotinados permitidos é fixada em $d$. Um caso particular deste problema é quando $d=2$. Neste caso temos o problema da mochila bidimensional em dois estágios (2TDK - 2-Stage Two-Dimensional Knapsack).

No modelo do problema de corte bidimensional em dois estágios, consideramos variáveis binárias $u_{l}(l=1, \ldots, p)$, que indicam se um objeto é utilizado ou não:

$$
u_{l}= \begin{cases}1, & \text { se o objeto } l \text { é utilizado }, \\ 0, & \text { caso contrário. }\end{cases}
$$

Usamos também variáveis binárias $x_{i k l}(k=1, \ldots, n, i=k, \ldots, n, l=1, \ldots, p)$, que indicam o objeto e a prateleira nos quais um item foi alocado:

$$
x_{i k l}= \begin{cases}1, & \text { se o item } i \text { é alocado à prateleira } k \text { do objeto } l, \\ 0, & \text { caso contrário. }\end{cases}
$$

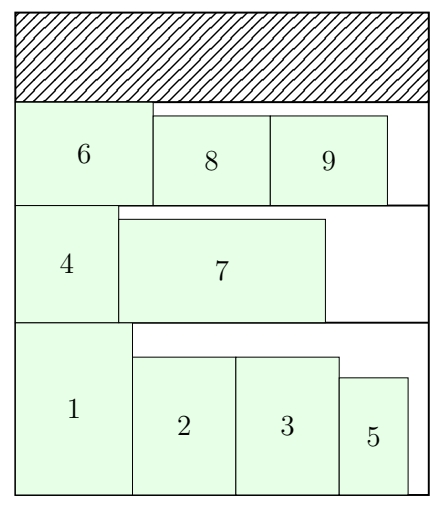

Figura 3.1: Formato da possível sobra aproveitável. Se a altura da região hachurada tem tamanho maior ou igual a $d_{\min }$, então é considerada uma sobra aproveitável, caso contrário é considerada desperdício. 
Dado um objeto $l$, uma prateleira é definida como uma fatia de um objeto com comprimento $W_{l}$ e altura correspondendo à altura do item mais alto cortado a partir dela. Mais ainda, todos os itens em uma prateleira possuem seu lado inferior sobre a base desta prateleira. $\mathrm{O}$ teto de uma prateleira, determinado pela aresta superior do item mais alto, define a base da prateleira seguinte. $\mathrm{O}$ conceito de prateleira pode ser exemplificado através da Figura 3.1. Nela, temos um objeto e três prateleiras a saber: 1,4 e 6 . Os itens $1,2,3$ e 5 estão na prateleira 1 , os itens 4 e 7 estão na prateleira 4 e os itens 6,8 e 9 na prateleira 6 . No modelo que apresentamos a seguir, consideramos que potencialmente podemos ter $n$ prateleiras em um objeto, cada uma definida por um item. Dizemos então que uma prateleira $k$ foi aberta, ou que é utilizada, se o item $k$ for o item mais à esquerda alocado nesta prateleira. Mais ainda, uma prateleira $k$ de um objeto $l$ está aberta se $x_{k k l}=1$. Podemos afirmar que toda solução viável para o problema de corte em dois estágios com sobras aproveitáveis é composta de prateleiras e que cada item alocado em uma prateleira pode ser cortado em no máximo dois estágios (mais o trimming). Podemos também dizer que qualquer solução ótima para o problema de corte em dois estágios com sobras aproveitáveis possui uma solução equivalente na qual o maior item em cada prateleira é também o item mais a esquerda.

Segue um modelo para o problema de corte em dois estágios:

$$
\begin{aligned}
& \underset{u, x}{\operatorname{Minimizar} f(u)} \quad=\sum_{l=1}^{p} u_{l} c_{l} \\
& \text { s.a } \quad \sum_{k=1}^{n} h_{k} x_{k k l} \leq H_{l} u_{l}, \quad l=1, \ldots, p, \\
& \sum_{i=k}^{n} w_{i} x_{i k l} \leq W_{l}, \quad k=1, \ldots, n, l=1, \ldots, p, \\
& \sum_{l=1}^{p} \sum_{k=1}^{i} x_{i k l}=1, \quad i=1, \ldots, n, \\
& x_{i k l} \quad \leq x_{k k l}, \quad k=1, \ldots, n, i=k+1, \ldots, n, l=1, \ldots, p, \\
& u_{l} \in\{0,1\}, \quad l=1, \ldots, p, \\
& x_{i k l} \in\{0,1\}, \quad k=1, \ldots, n, i=k, \ldots, n, l=1, \ldots, p .
\end{aligned}
$$

A restrição (3.4) impõe que, para cada objeto, a soma das alturas de cada prateleira aberta não supere a altura do objeto em questão. A restrição (3.5) garante que, para cada objeto, a soma das larguras dos itens alocados a cada prateleira não supere a largura do objeto em questão. A restrição (3.6) impõe que a demanda seja satisfeita e (3.7) garante que um item só pode ser atribuído a uma prateleira caso ela tenha sido aberta. As restrições (3.8) e (3.9) garantem que as variáveis $u_{l}$ e $x_{i j k}$ sejam binárias.

O modelo (3.8-3.9) descreve o problema de corte bidimensional em 2-estágios de itens retangulares. Na seção seguinte mostramos como incorporar a maximização das sobras aproveitáveis no modelo (3.3-3.9).

\subsection{Considerando as sobras aproveitáveis}

Apresentamos nesta seção o modelo para o problema de corte bidimensional em dois estágios com sobras aproveitáveis. Conforme dissemos anteriormente, sobras aproveitáveis são as sobras resultantes dos cortes longitudinais que tiverem altura maior ou igual a $d_{\min }$. Já que desejamos uma solução que minimize o custo associado aos objetos utilizados e, dentre as possíveis combinações de padrões de corte que contemplam custo mínimo, uma que maximize a soma dos valores das 
sobras aproveitáveis, consideramos que o mais natural seria propor um modelo de programação em dois níveis.

Um modelo de programação em dois níveis é um modelo de otimização no qual desejamos maximizar ou minimizar uma função objetivo com algumas das variáveis do problema restritas a serem solução de um outro problema de otimização. O modelo a seguir retrata um modelo de programação em dois níveis genérico, de acordo com [Dempe02]:

$$
\begin{aligned}
& \underset{\bar{x} \in X}{\operatorname{Maximizar}} f^{s}(\bar{x}, \bar{y}) \\
& \text { s.a } \\
& \quad g^{s}(\bar{x}, \bar{y}) \leq 0 \\
& h^{s}(\bar{x}, \bar{y})=0 \\
& \bar{y} \in \underset{\bar{z} \in Y}{\operatorname{argmin}} f^{i}(\bar{x}, \bar{z}) \\
& \text { s.a } \quad g^{i}(\bar{x}, \bar{z}) \leq 0 \\
& \\
& h^{i}(\bar{x}, \bar{z})=0
\end{aligned}
$$

No modelo (3.10), temos $X \subseteq \mathbb{R}^{n^{x}}$ e $Y \subseteq \mathbb{R}^{n^{y}}$. As funções $f^{s}, f^{i}: \mathbb{R}^{n^{x}} \times \mathbb{R}^{n^{y}} \rightarrow \mathbb{R}$ são chamadas respectivamente de função objetivo do nível superior e função objetivo do nível inferior. As inequações $g^{s}(\bar{x}, \bar{y}) \leq 0, h^{s}(\bar{x}, \bar{y})=0$ e $g^{i}(\bar{x}, \bar{y}) \leq 0, h^{i}(\bar{x}, \bar{y})=0$, são chamadas respectivamente de restrições do nível superior e restrições do nível inferior, tais que $g^{s}: \mathbb{R}^{n^{x}} \times \mathbb{R}^{n^{y}} \rightarrow \mathbb{R}^{m^{g^{s}}}$, $g^{i}: \mathbb{R}^{n^{x}} \times \mathbb{R}^{n^{y}} \rightarrow \mathbb{R}^{m^{g^{i}}}, h^{s}: \mathbb{R}^{n^{x}} \times \mathbb{R}^{n^{y}} \rightarrow \mathbb{R}^{m^{h^{s}}}$ e $h^{i}: \mathbb{R}^{n^{x}} \times \mathbb{R}^{n^{y}} \rightarrow \mathbb{R}^{m^{h^{i}}}$. Segundo [Vicente94], a formulação (3.10) pode ser denominada Problema de Stackelberg Estático (Static Stackelberg Problem). A formulação (3.10) permite duas abordagens, a abordagem otimista e a pessimista. A abordagem otimista pressupõe que há alguma afinidade entre o tomador de decisão do problema do nível superior e o tomador de decisão do problema do nível inferior. Desta forma, se o conjunto de soluções do problema do nível inferior não for unitário, será escolhida a solução que maximiza o valor de $f^{s}(\bar{x}, \bar{y})$. A abordagem pessimista pode ser interpretada como se não houvesse uma cooperação entre os tomadores de decisão do nível superior e inferior. Dessa forma, a solução do problema de programação em dois níveis seria aquela que minimizasse a perda causada pela pior escolha dentre as possíveis soluções do problema do nível inferior. Em todos os modelos de programação em dois níveis apresentados neste trabalho, consideramos uma abordagem otimista.

O modelo que propomos a seguir, para o problema de corte bidimensional em dois estágios com sobras aproveitáveis, consiste em um modelo de programação em dois níveis com variáveis inteiras e contínuas e com funções objetivo e restrições todas lineares. Antes de apresentar o modelo, explicamos como modelar as sobras aproveitáveis. Consideremos $s_{l}$ a altura da sobra do objeto $l=1, \ldots, p$. Desejamos que a sobra de um determinado objeto $l$ seja considerada aproveitável se sua altura $s_{l}$ for tal que $s_{l} \geq d_{\text {min. }}$. Desejamos também maximizar o valor das sobras aproveitáveis. Vamos definir esse valor como sendo o valor da área da sobra aproveitável. Se uma sobra não for aproveitável, seu valor será zero. Para modelar as sobras aproveitáveis podemos, então, criar uma função que assuma o valor da área da sobra quando ela for aproveitável e zero em caso contrário. Denominamos esta função de $\bar{s}\left(s_{l}\right)$ e a definimos da seguinte forma:

$$
\bar{s}\left(s_{l}\right)= \begin{cases}W_{l} s_{l}, & \text { se } s_{l} \geq d_{\min } \\ 0, & \text { caso contrário. }\end{cases}
$$

Podemos escrever (3.11) como restrições de um MIP usando a técnica do M-grande, como veremos a seguir. Para simplificar a explicação, apresentamos o modelo em dois níveis para o problema de corte bidimensional em dois estágios com sobras e em seguida detalhamos como transformar (3.11) em restrições de um MIP, bem como os demais detalhes do modelo: 


$$
\begin{aligned}
& \underset{z, T}{\operatorname{aaximizar}} F(T)=\sum_{l=1}^{p} T_{l} \\
& \text { s.a } \quad d_{\min } \leq s_{l}+M_{l} \\
& s_{l} \leq d_{\min }+M_{l}\left(1-z_{l}\right), \\
& T_{l} \quad \leq s_{l} W_{l}+\bar{M}_{l} z_{l} \\
& \left.T_{l} \leq \bar{M}_{l}\left(1-z_{l}\right), \quad\right\} l=1, \ldots, p \\
& T_{l} \quad \geq 0, \\
& z_{l} \in\{0,1\}, \\
& (u, x, s) \in \underset{u^{\prime}, x^{\prime}, s^{\prime}}{\operatorname{argmin}} f\left(u^{\prime}\right)=\sum_{l=1}^{p} u_{l}^{\prime} c_{l} \\
& \text { s.a } \quad \sum_{k=1}^{n} h_{k} x_{k k l}^{\prime}+s_{l}^{\prime} \quad=H_{l} u_{l}^{\prime} \\
& \sum_{i=k}^{n} w_{i} x_{i k l}^{\prime} \quad \leq W_{l}, \quad k=1, \ldots, n, l=1, \ldots, p \\
& x_{i k l}^{\prime} \quad \leq x_{k k l}^{\prime} \\
& k=1, \ldots, n, \\
& i=k+1, \ldots, n, l=1, \ldots, p, \\
& \sum_{l=1}^{p} \sum_{k=1}^{i} x_{i k l}^{\prime} \quad=1, \quad i=1, \ldots, n, \\
& s_{l}^{\prime} \quad \geq 0, \\
& u_{l}^{\prime} \in\{0,1\}, \quad l=1, \ldots, p \text {, } \\
& x_{i k l}^{\prime} \in\{0,1\}, \quad k=1, \ldots, n \text {. } \\
& i=k, \ldots, n l=1, \ldots, p \text {, }
\end{aligned}
$$

O problema do nível superior (3.12-3.18) impõe que a soma das áreas das sobras aproveitáveis nos objetos utilizados seja maximizada e o problema do nível inferior (3.19-3.26) impõe que seja minimizado o custo dos objetos. As variáveis $s_{l}(l=1, \ldots, p)$ indicam a altura das sobras aproveitáveis de cada objeto. As restrições (3.13-3.16), juntamente com a função objetivo (3.12) modelam $\bar{s}\left(s_{l}\right)$ definida em (3.11) através da técnica do M grande. Para isso, precisamos de constantes $M_{l}$ e $\bar{M}_{l}$ tais que $H_{l} \leq M_{l}$ e $H_{l} W_{l} \leq \bar{M}_{l}(l=1, \ldots, p)$ e utilizamos uma variável binária $z_{l}$ (por objeto). Se a variável $z_{l}=0$, temos que a altura $s_{l}$ da sobra correspondente deve ser maior ou igual que $d_{\min }$ e a variável $T_{l}$ deve ter valor correspondente à área da sobra aproveitável (observe que (3.15) juntamente com a função objetivo garantem isso). No caso em que $z_{l}=1$, temos que deve valer $s_{l} \leq d_{\min }$ e $T_{l}=0$ (observe (3.16-3.17)), ou seja, o objeto $l$ não tem sobra aproveitável. No caso de $s_{l}$ ser igual a $d_{\min }$, pelo fato de maximizarmos o somatório $\sum_{l=1}^{p} T_{l}$, teremos $z_{l}=0$. A inequação (3.17) apenas impõem a não negatividade da variável $T_{l}$. Já (3.18) apenas restringe $z_{l}$ 
como sendo uma variável binária. A restrição (3.19) impõe que $(u, x, s)$ seja solução de um problema de otimização, chamado de problema do nível inferior, no qual desejamos minimizar o custo dos objetos utilizados para satisfazer a demanda de corte. O problema do nível inferior, formado pela função objetivo (3.19) e pelas restrições (3.20-3.26), é essencialmente o mesmo definido em (3.3-3.9), com exceção das restrições (3.20) e (3.24). A restrição (3.20) difere de (3.4) pela adição do termo $s_{l}$, incorporando a dimensão da sobra aproveitável em cada objeto. A restrição (3.24) pede a não negatividade de $s_{l}$. Dessa forma, a restrição (3.20) impõe que a soma das alturas das prateleiras abertas mais a altura da sobra aproveitável, em um objeto utilizado, seja igual a altura do objeto em questão.

Uma técnica comumente aplicada para resolver problemas de programação em dois níveis é realizar algum tipo de reformulação do modelo em dois níveis para um modelo de otimização que sabemos resolver. A seguir apresentamos uma reformulação do modelo em dois níveis em um MIP. Considerando que a função objetivo do problema do nível inferior (3.19) assume apenas valores inteiros (as variáveis $u_{l}, l=1, \ldots, p$, são binárias e os custos $c_{l}, l=1, \ldots p$, dos objetos são inteiros, por hipótese) e independe das variáveis do problema do nível superior, o modelo (3.12-3.26) pode ser reformulado como um modelo de programação linear com variáveis inteiras da seguinte forma:

$$
\begin{aligned}
& \underset{z, T, s, u, x}{\operatorname{Minimizar}} \bar{F}(T, u)=\sum_{l=1}^{p} u_{l} c_{l}-\frac{\sum_{l=1}^{p} T_{l}}{\sum_{l=1}^{p} W_{l} H_{l}} \\
& \text { s.a } \quad T_{l} \\
& \leq s_{l} W_{l}+\bar{M}_{l} z_{l}, \\
& l=1, \ldots, p, \\
& T_{l} \quad \leq \bar{M}_{l}\left(1-z_{l}\right) \text {, } \\
& l=1, \ldots, p, \\
& d_{\text {min }} \leq s_{l}+M_{l} z_{l}, \\
& l=1, \ldots, p, \\
& s_{l} \quad \leq d_{\min }+M_{l}\left(1-z_{l}\right), \\
& l=1, \ldots, p, \\
& T_{l} \quad \geq 0, \\
& l=1, \ldots, p, \\
& s_{l} \quad \geq 0 \text {, } \\
& l=1, \ldots, p, \\
& \sum_{k=1}^{n} h_{k} x_{k k l}+s_{l}=H_{l} u_{l} \\
& l=1, \ldots, p, \\
& \sum_{i=k}^{n} w_{i} x_{i k l} \quad \leq W_{l}, \\
& k=1, \ldots, n, l=1, \ldots, p, \\
& \sum_{l=1}^{p} \sum_{k=1}^{i} x_{i k l} \quad=1 \\
& i=1, \ldots, n, \\
& x_{i k l} \leq x_{k k l}, \\
& z_{l}, u_{l} \in\{0,1\}, \\
& x_{i k l}^{\prime} \in\{0,1\} \text {, } \\
& \begin{aligned}
k=1, \ldots, n, i=k+1, \ldots, n, l & =1, \ldots, p \\
l & =1, \ldots, p . \\
k=1, \ldots, n, i=k, \ldots, n, l & =1, \ldots, p
\end{aligned}
\end{aligned}
$$

Observe que a função objetivo (3.27) é composta pelas funções objetivo dos níveis inferior e superior de (3.12-3.26), sendo que a função objetivo do problema do nível superior (3.12) foi normalizada de forma a assumir valores dentro do intervalo $[0,1)$. Observe também que as restrições (3.28-3.39) correspondem exatamente às restrições (3.13-3.18) e (3.20-3.26). Chamamos, de agora em diante, o modelo definido por (3.27-3.39), de modelo $\mathcal{M}_{0}^{D E}$.

Considerando $\left(T^{*}, u^{*}\right)$ uma solução ótima de $\mathcal{M}_{0}^{D E}$, o valor de $\bar{F}\left(T^{*}, u^{*}\right)$ será inteiro caso não haja sobras aproveitáveis. No caso de existirem sobras aproveitáveis, o valor de $\bar{F}\left(T^{*}, u^{*}\right)$ será racional. A partir do valor de $\bar{F}\left(T^{*}, u^{*}\right)$, é possível obter os valores correspondentes de (3.12) e 
(3.19) da seguinte forma:

$$
\begin{aligned}
f\left(u^{*}\right) & =\quad\left\lceil\bar{F}\left(T^{*}, u^{*}\right)\right\rceil, \\
F\left(T^{*}\right) & =\left(\left\lceil\bar{F}\left(T^{*}, u^{*}\right)\right\rceil-\bar{F}\left(T^{*}, u^{*}\right)\right) \sum_{l=1}^{p} W_{l} H_{l} .
\end{aligned}
$$

É verdade que toda solução ótima de $\mathcal{M}_{0}^{D E}$ é uma solução ótima de (3.12-3.26). Por outro lado, é importante notar que apesar de toda solução viável de (3.12-3.26) ser uma solução viável de $\mathcal{M}_{0}^{D E}$, o contrário não necessariamente é verdade.

Note também o fato de que atribuir um custo $c_{l}=0$ a um objeto o torna parte da solução (ou seja, temos $u_{l}=1$ ). Isso acontece porque se $c_{l}=0$, a parcela $u_{l} c_{l}$ de (3.27) será nula para qualquer valor de $u_{l}$, logo, podemos ter $u_{l}=1$ sem causar acréscimo no valor da função objetivo. Assim, se pelo menos um item puder ser cortado a partir do objeto $l$, claramente a solução ótima conterá um padrão de corte $\operatorname{com} l$, ou seja $u_{l}=1$ (se nenhum item puder ser cortado a partir do objeto $l$ ele ainda poderá estar na solução inteiramente como sobra aproveitável, caso sua altura satisfaça a condição para uma sobra ser aproveitável). Dessa forma podemos priorizar a utilização de um objeto definindo seu custo como nulo. Isso pode ser interessante para priorizar o uso das sobras aproveitáveis na geração dos padrões de corte, evitando que as mesmas se acumulem em estoque, por exemplo. Analisando o tamanho do modelo $\mathcal{M}_{0}^{D E}$, notamos que ele possui $2 p$ variáveis reais, $2 p+\frac{n p}{2}+\frac{n^{2}}{2}$ variáveis binárias e $5 p+\frac{n p}{2}+n+\frac{n^{2} p}{2}$ restrições.

Para uma avaliação inicial de $\mathcal{M}_{0}^{D E}$ optamos, primeiramente, por fazer uma implementação em AMPL . Segue o código:

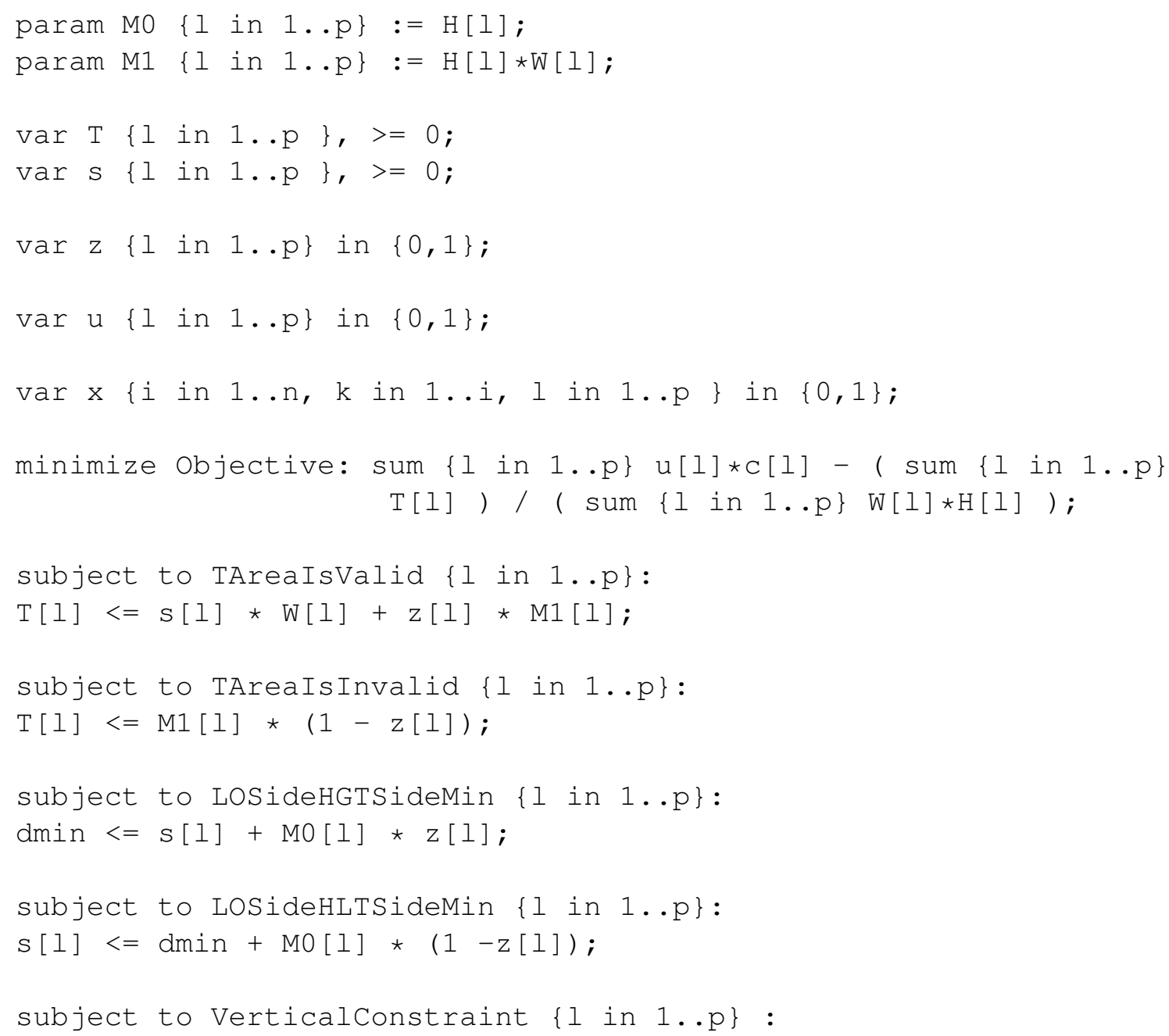


$(\operatorname{sum}\{\mathrm{k}$ in $1 \ldots \mathrm{n}\} \mathrm{h}[\mathrm{k}] \star \mathrm{x}[\mathrm{k}, \mathrm{k}, 1])+\mathrm{s}[1]<=\mathrm{H}[1] \star \mathrm{u}[1]$;

subject to HorizontalConstraint $\{1$ in $1 \ldots \mathrm{p}, \mathrm{k}$ in $1 . . \mathrm{n}\}$ :

( $\operatorname{sum}\{i$ in $k \ldots n\} w[i] \star x[i, k, l])<=W[1] ;$

subject to SatisfyDemand $\{i$ in $1 \ldots n\}$ :

sum $\{1$ in $1 \ldots \mathrm{p}\}$ sum $\{\mathrm{k}$ in $1 \ldots i\} \times[i, k, 1]=1$;

subject to shelfItemConstraint $\{1$ in $1 \ldots \mathrm{p}, \mathrm{k}$ in $1 \ldots \mathrm{n}, \mathrm{i}$ in $\mathrm{k}+1 . . \mathrm{n}\}$ : $\mathrm{x}[\mathrm{i}, \mathrm{k}, \mathrm{l}]<=\mathrm{x}[\mathrm{k}, \mathrm{k}, \mathrm{l}]$;

Consideramos a instância do problema de corte em dois estágios com sobras aproveitáveis definida pelos seguintes parâmetros: $n=30, p=2, w_{i}=2$ e $h_{i}=1$, para $i=1, \ldots, n, W_{l}=6$ e $H_{l}=7$ para $l=1, \ldots, p, c_{1}=42$ e $c_{2}=1$. Segue o código AMPL para esta instância:

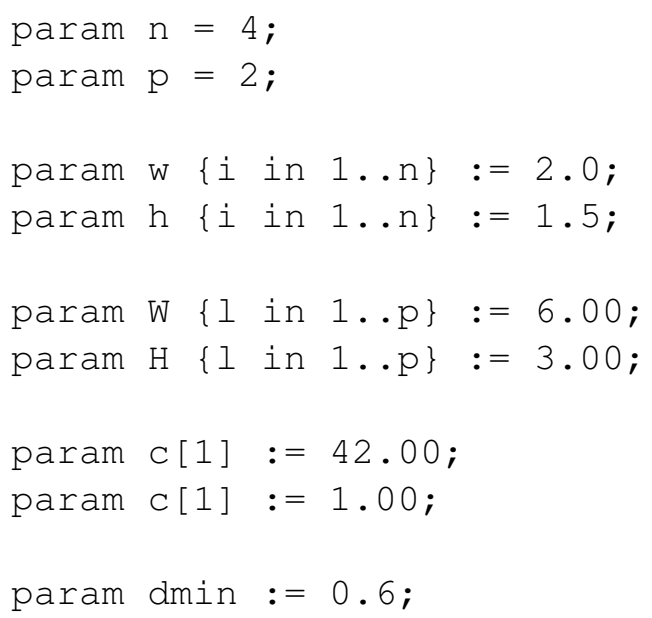

Ao resolver esta instância com o CPLEX, obtivemos a solução ilustrada na Figura 3.2. Nela notamos o inconveniente das sobras estarem distribuídas nos dois objetos em vez de se concentrar em apenas um. Isso nos motivou a modificar o modelo $\mathcal{M}_{0}^{D E}$ de forma a priorizar a geração de

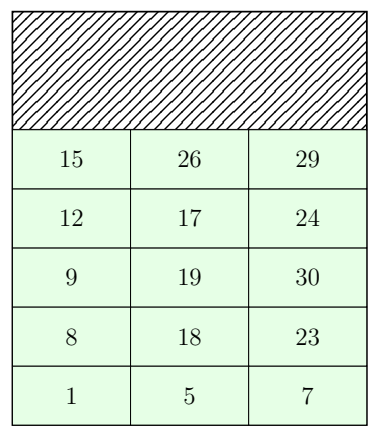

\begin{tabular}{|c|c|c|}
\hline 21 & 27 & 28 \\
\hline 13 & 14 & 22 \\
\hline 6 & 11 & 25 \\
\hline 4 & 10 & 20 \\
\hline 2 & 3 & 16 \\
\hline
\end{tabular}

Figura 3.2: Ilustração da solução para a instância definida pelos parâmetros: $n=30, p=2, w_{i}=2 e$ $h_{i}=1$, para $i=1, \ldots, n, W_{l}=6$ e $H_{l}=7$ para $l=1, \ldots, p, c_{1}=42$ e $c_{2}=1$. Note o inconveniente das sobras estarem distribuídas nos dois objetos em vez de concentradas em apenas um.

sobras aproveitáveis em objetos com custo mais alto. Em particular, na solução ilustrada pela Figura 3.2, gostaríamos que toda a sobra fosse concentrada na placa mais cara. Para tal, decidimos inserir o custo de cada objeto na parcela da função objetivo responsável por maximizar a área das 
sobras de forma que a função objetivo fique assim:

$$
\tilde{F}(T, u)=\sum_{l=1}^{p} u_{l} c_{l}-\frac{\sum_{l=1}^{p} T_{l} c_{l}}{\sum_{l=1}^{p} W_{l} H_{l} c_{l}} .
$$

Dessa forma podemos definir um novo modelo MIP reformulado que consiste em minimizar (3.42) sujeito às inequações (3.28-3.39). Chamamos este modelo de $\mathcal{M}_{1}^{D E}$. Considerando os mesmos dados do experimento descrito anteriormente, porém agora utilizando o modelo $\mathcal{M}_{1}^{D E}$, obtivemos a solução ilustrada na Figura 3.3. Conforme esperado, os itens foram organizados de forma a termos uma única sobra na placa com valor maior.

Anteriormente sugerimos que para priorizar o uso de alguns objetos, poderíamos modelá-los com um custo nulo. Considerando (3.42) como função objetivo, ao modelar um objeto com custo nulo, deixamos de considerar sua sobra aproveitável. Para contornar esse problema, podemos considerar constantes $\hat{c}_{l}$ representando a prioridade ou o custo da sobra aproveitável de um objeto $l$. Ou seja, $\hat{c}_{l}$ pode ser usado para definir preferência por sobra em um determinado objeto em detrimento de outro, mesmo no caso de objetos com custo $c_{l}=0$. Para isso, modificamos (3.42) da seguinte maneira:

$$
\hat{F}(T, u)=\sum_{l=1}^{p} u_{l} c_{l}-\frac{\sum_{l=1}^{p} T_{l} \hat{c}_{l}}{\sum_{l=1}^{p} W_{l} H_{l} \hat{c}_{l}} .
$$

Assim teremos um conjunto de custos $c_{l}$ associado ao uso de cada objeto $l$ e um valor ou custo $\hat{c}_{l}$ associado à sobra aproveitável no objeto $l$. Note que isso seria o equivalente a multiplicar a função objetivo (3.12) por $\hat{c}_{l}$. Dessa forma estamos redefinindo o que consideramos valor da sobra aproveitável. Inicialmente consideramos que tal valor seria o valor da área da sobra quando uma sobra é aproveitável ou zero em caso contrário. Agora consideramos que o valor de uma sobra de um objeto $l$ é o valor de sua área multiplicado por $\hat{c}_{l}$,quando esta sobra for aproveitável, e zero, caso contrário. Combinando (3.43) com as restrições (3.28-3.39) definimos o modelo $\mathcal{M}_{2}^{D E}$.

Apresentamos nesta seção três modelos de programação inteira mista para o problema de corte em dois estágios com sobras aproveitáveis. Na seção seguinte apresentamos mais um modelo para o problema de corte bidimensional em dois estágios (sem sobras aproveitáveis) que tem como característica considerar a ideia de tipos ou grupos de itens iguais, em vez de modelar cada item separadamente.

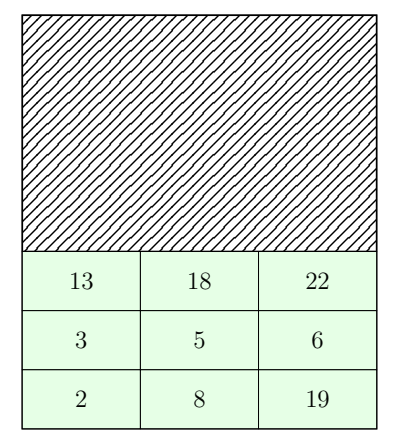

\begin{tabular}{|c|c|c|}
\hline 25 & 27 & 30 \\
\hline 23 & 28 & 29 \\
\hline 17 & 24 & 26 \\
\hline 11 & 20 & 21 \\
\hline 7 & 12 & 15 \\
\hline 4 & 10 & 16 \\
\hline 1 & 9 & 14 \\
\hline
\end{tabular}

Figura 3.3: Ilustração da solução para a instância definida pelos parâmetros: $n=30, p=2, w_{i}=2 e$ $h_{i}=1$, para $i=1, \ldots, n, W_{l}=6$ e $H_{l}=7$ para $l=1, \ldots, p, c_{1}=42$ e $c_{2}=1$, considerando (3.43) como função objetivo. 


\subsection{Outro modelo para o problema de corte dois estágios}

Em [Lodi03] é apresentado um outro modelo para o problema da mochila bidimensional, referenciado como modelo $\mathrm{M} 2$, que trata itens iguais como sendo itens de um mesmo tipo, agrupandoos. Nesta seção apresentamos uma versão modificada do modelo M2 apresentado em [Lodi03] para resolver o problema de corte bidimensional em dois estágios (sem sobras aproveitáveis).

No modelo que apresentamos a seguir temos $n$ itens de $m$ tipos distintos. Um item do tipo $i$ possui dimensões horizontal e vertical dadas por $\bar{w}_{i}$ e $\bar{h}_{i}$, respectivamente. Supomos que $\bar{h}_{1} \geq$ $\bar{h}_{2} \geq \ldots \geq \bar{h}_{m}$. A quantidade de itens do tipo $i$ é dada por $b_{i}$. São consideradas ainda novas constantes a saber: $\alpha_{i}(i=1, \ldots, m)$ e $\beta_{k}(k=1, \ldots, n)$. Dado um item do tipo $i$, ele pode ser cortado de qualquer prateleira no intervalo $\left[1, \alpha_{i}\right]$, tal que $\alpha_{0}=0$ e $\alpha_{i}=\sum_{s=1}^{i} b_{s}(i=1, \ldots, m)$. A constante $\alpha_{i}$ indica o maior índice de prateleira que pode alocar itens do tipo $i$. Podemos ainda interpretar o intervalo $\left[\alpha_{i-1}+1, \alpha_{i}\right]$ como os índices das prateleiras caracterizadas por itens do tipo $i$. Por outro lado, uma prateleira $k$ pode alocar itens dos tipos $\left[\beta_{k}, m\right]$, tal que $\beta_{k}=\min \left\{i: \alpha_{i} \geq k\right\}$ $(k=1, \ldots, n)$. A constante $\beta_{k}$ pode ser vista como o índice do tipo de item de maior altura (menor índice) que pode ser alocado na prateleira $k$ ou ainda como o índice do tipo do item que caracteriza a prateleira. Note que temos uma prateleira para cada item.

As variáveis $x_{i k l}(k=1, \ldots, n, i=k+1, \ldots, n, l=1, \ldots, p)$ são utilizadas neste modelo, porém com um significado um pouco diferente. São inteiras e relacionam itens e prateleiras da seguinte forma:

$$
x_{i k l}= \begin{cases}\text { quantidade total de itens do tipo } i \text { alocados à prateleira } k \text { do objeto } l, & \text { se } i \neq \beta_{k}, \\ \text { quantidade adicional }{ }^{1} \text { de itens do tipo } i \text { alocados à prateleira } k \text { do objeto } l & \text { se } i=\beta_{k} .\end{cases}
$$

O modelo também considera variáveis $q_{k l}(k=1, \ldots, n, l=1, \ldots, p)$, binárias, que indicam se uma prateleira é aberta (utilizada) ou não:

$$
q_{k l}= \begin{cases}1, & \text { se a prateleira } k \text { do objeto } l \text { é aberta } \\ 0, & \text { caso contrário. }\end{cases}
$$

As demais variáveis e constantes permanecem iguais ao problema do nível inferior (3.19-3.26).

O nosso modelo baseado no modelo M2 de [Lodi03] para o problema de corte bidimensional em dois estágios segue:

$$
\begin{aligned}
& \underset{u, x}{\operatorname{Minimizar}} f(u)=\sum_{l=1}^{p} u_{l} c_{l} \\
& \text { s.a } \quad \sum_{k=1}^{n} \bar{h}_{\beta_{k}} q_{k l}+s_{l}=H_{l} u_{l}, \quad l=1, \ldots, p, \\
& \sum_{i=\beta k}^{m} \bar{w}_{i} x_{i k l} \leq\left(W_{l}-\bar{w}_{\beta_{k}}\right) q_{k l}, \quad k=1, \ldots, n, l=1, \ldots, p, \\
& \sum_{l=1}^{p}\left(\sum_{k=1}^{\alpha_{i}} x_{i k l}+\sum_{k=\alpha_{i-1}+1}^{\alpha_{i}} q_{k l}\right)=b_{i}, i=1, \ldots, m, \\
& \sum_{l=1}^{p} q_{k l} \leq 1 \quad k=1, \ldots, n
\end{aligned}
$$

\footnotetext{
${ }^{1}$ Descontando o item que abre/caracteriza a prateleira.
} 


$$
\begin{array}{ll}
\sum_{l=1}^{p} q_{k+1, l} \leq \sum_{l=1}^{p} q_{k l} & k=1, \ldots, m, k \in\left[\alpha_{i-1}+1, \alpha_{i}-1\right] \\
\sum_{s=k}^{\alpha_{i}} x_{i s l} \leq b_{i}-\left(k-\alpha_{i-1}\right) & , i=1, \ldots, m, \\
& k \in\left[\alpha_{i-1}+1, \alpha_{i}\right], l=1, \ldots, p, \\
x_{i k l} \leq b_{i}, & i=1, \ldots, m, \\
& k \in\left[1, \alpha_{i}\right], l=1, \ldots, p \\
u_{l} \in\{0,1\}, & l=1, \ldots, p, \\
q_{k l} \in\{0,1\}, & k=1, \ldots, n, l=1, \ldots, p, \\
x_{i k l} \in \mathbb{N}_{0}, & i=1, \ldots, m, k \in\left[1, \alpha_{i}\right], l=1, \ldots, p .
\end{array}
$$

A restrição (3.47) impõe que, para cada objeto, a soma das alturas de cada prateleira aberta mais a sobra seja igual à altura do objeto em questão. A restrição (3.48) impõe que, para cada objeto, a soma das larguras dos itens alocados a cada prateleira não supere a largura do objeto em questão. A restrição (3.49) impõe que a demanda seja satisfeita. A restrição (3.50) garante que cada prateleira só pode ser aberta uma única vez (não podemos ter uma prateleira $k$ aberta em duas placas diferentes). A restrição (3.51) procura eliminar a simetria forçando que, dentre prateleiras iguais, sejam abertas primeiro as correspondentes aos itens de índice menor. A restrição (3.52), embora redundante em termos da formulação MIP, foi utilizada em [Lodi03] com o objetivo de que o modelo relaxado (obtido ao eliminar-se as restrições de integralidade) forneça limitantes inferiores de melhor qualidade.

O modelo (3.46-3.52) pode ser utilizado na formulação de um novo modelo de programação em dois níveis para o problema de corte bidimensional em dois estágios com sobras aproveitáveis. Para isso, basta substituir (3.20-3.26) por (3.47-3.52) compondo o modelo (3.12-3.19, 3.47-3.52). Da mesma forma, podemos escrever a reformulação MIP deste novo modelo de programação em dois níveis, considerando o modelo $\mathcal{M}_{1}^{D E}$ e substituindo (3.34-3.39) por (3.47-3.52) compondo o modelo $\mathcal{M}_{3}^{D E}$.

\begin{tabular}{|l|c|c|}
\hline & Modelo $\mathcal{M}_{1}^{D E}$ & Modelo $\mathcal{M}_{3}^{D E}$ \\
\hline Variáveis reais & $2 p$ & $2 p$ \\
Variáveis binárias & $2 p+\frac{n p}{2}+\frac{n^{2} p}{2}$ & $2 p+n p$ \\
Variáveis inteiras & 0 & $p \sum_{k=0}^{m} \alpha_{k}$ \\
Restrições & $5 p+\frac{n p}{2}+n+\frac{n^{2} p}{2}$ & $5 p+n p+3 n+p \sum_{k=0}^{m} \alpha_{k}$ \\
\hline
\end{tabular}

Tabela 3.1: Comparação de $\mathcal{M}_{1}^{D E}$ com $\mathcal{M}_{3}^{D E}$ considerando a quantidade de variáveis e restrições de cada modelo. Apesar de variáveis binárias serem um caso particular de variáveis inteiras, nesta comparação optamos por considerá-las em separado.

A Tabela 3.1 resume o número de variáveis contínuas, binárias e inteiras e o número de restrições dos modelos $\mathcal{M}_{1}^{D E}$ e $\mathcal{M}_{3}^{D E}$. É importante notar que apesar de variáveis binárias serem um caso particular de variáveis inteiras, optamos por considerá-las em separado. Na tabela é possível notar que ambos os modelos possuem a mesma quantidade de variáveis contínuas, mas diferem em termos das quantidades de variáveis binárias e inteiras. $\mathrm{O}$ modelo $\mathcal{M}_{1}^{D E}$ possui $O\left(n^{2} p\right)$ variáveis binárias enquanto que $\mathcal{M}_{3}^{D E}$ possui apenas $O(n p)$. Em termos de variáveis inteiras, $\mathcal{M}_{1}^{D E}$ não possui e $\mathcal{M}_{3}^{D E}$ possui $p \sum_{k=0}^{m} \alpha_{k}$. Analisando $\sum_{k=0}^{m} \alpha_{k}$, temos os seguintes casos extremos:

- se $m=1$, então $\sum_{k=0}^{m} \alpha_{k}=n$, 
- se $m=n$ e $\bar{h}_{1}>\bar{h}_{2}>\ldots>\bar{h}_{m}$, então $\sum_{k=0}^{m} \alpha_{k}=\frac{n^{2}+n}{2}$,

- se $m=n$ e $\bar{h}_{1}=\bar{h}_{2}=\ldots=\bar{h}_{m}$, então $\sum_{k=0}^{m} \alpha_{k}=m n=n^{2}$.

Dessa forma, no pior caso, $\mathcal{M}_{3}^{D E}$ possui $O\left(n^{2} p\right)$ variáveis inteiras. Considerando as restrições podemos observar que $\mathcal{M}_{1}^{D E}$ possui $O\left(n^{2} p\right)$ restrições e que $\mathcal{M}_{3}^{D E}$ possui, no pior caso, também $O\left(n^{2} p\right)$ restrições. Vale observar que na maioria dos casos esperamos que $m$ seja um número pequeno em relação a $n$. Dessa forma, o modelo $\mathcal{M}_{3}^{D E}$ deve possuir uma quantidade de variáveis inteiras e restrições proporcional a $n p$ e portanto, esperamos que seja mais fácil de resolver do que $\mathcal{M}_{1}^{D E}$.

Na seção seguinte apresentamos e analisamos alguns experimentos numéricos com os modelos apresentados.

\subsection{Experimentos numéricos}

Para a realização de experimentos numéricos, desenvolvemos um gerador aleatório de instâncias baseado nas ideias do procedimento utilizado em [Hifi01]. O gerador recebe nove parâmetros a saber:

- $s$ : semente para a geração de números aleatórios,

- $m_{\min }^{p}$ e $m_{\max }^{p}$ : limitantes inferior e superior para a quantidade de tipos de objetos,

- $D_{\min }$ e $D_{\max }$ : limitantes inferior e superior para a dimensão horizontal e vertical de cada objeto,

- $m_{\min }$ e $m_{\max }$ : limitantes inferior e superior para a quantidade de tipos de itens,

- $n_{\min }$ e $n_{\max }$ : limitantes inferior e superior para a quantidade de itens de cada tipo.

Inicialmente o gerador sorteia de forma aleatória a quantidade de tipos de objetos distintos no intervalo $\left[m_{\min }^{p}, m_{\mathrm{max}}^{p}\right]$. Em seguida gera as dimensões $W_{1}, \ldots, W_{p}$ e $H_{1}, \ldots, H_{p}$ aleatoriamente no intervalo $\left[D_{\min }, D_{\max }\right]$. A quantidade de tipos de itens é escolhida aleatoriamente no intervalo $\left[m_{\min }, m_{\max }\right]$. A quantidade de itens de cada tipo é escolhida de forma aleatória no intervalo $\left[n_{\min }, n_{\max }\right]$ e as dimensões de cada tipo de item são escolhidas de forma aleatória no intervalo $[0.05 D, 0.4 D]$ ( $D=W_{\max }$ ou $D=H_{\max }$, dependendo de qual dimensão, horizontal ou vertical, respectivamente, está sendo sorteada). No caso das dimensões dos itens, caso seja gerado um item que não caiba em pelo menos um objeto, ele é descartado e gera-se outro aleatoriamente até que obtenha-se um item que caiba em pelo menos um objeto. A quantidade de objetos de cada tipo é calculada empacotando-se de forma trivial todos os itens em um só tipo de objeto (deixando-se de fora os itens que não cabem em cada tipo de objeto) e tomando-se a quantidade de objetos utilizada nesse empacotamento. Por empacotar trivialmente nos referimos ao procedimento de escolher aleatoriamente um objeto e colocar os itens um a um da esquerda para a direita e de baixo para cima até que o objeto seja preenchido completamente, repetindo o mesmo com outro objeto até que todos os itens sejam empacotados. Neste procedimento, a ordem de seleção dos itens a serem empacotados é arbitrária, utilizamos a ordem em que os itens são gerados. Vale enfatizar que todas as escolhas aleatórias foram realizadas utilizando um gerador de números pseudoaleatórios com distribuição uniforme.

O gerador e os modelos $\mathcal{M}_{1}^{D E}$ e $\mathcal{M}_{3}^{D E}$ foram implementados em $\mathrm{C}++$. No caso dos modelos utilizamos o CPLEX 12.1 e a Concert Techonology 2.9. Com relação às opções de configuração do CPLEX, definimos a variável PreInd, referente ao presolve, como false e mudamos a 
variável EpGap, referente a precisão relativa exigida pelo solver para considerar um ponto viável como solução, para $10^{-7}$ (o valor definido como padrão é $10^{-4}$ ). ${ }^{2}$ Também modificamos o tempo limite para resolução de uma instância para 3h (definimos o parâmetro Ti Lim como 10800) e pedimos para que o CPLEX armazene a árvore Branch \& Bound em disco caso ela exceda 3000MB (definimos NodeFileInd como 3 e WorkMem como 3000). Os programas foram compilados utilizando o compilador G++ 4.4.3 da GCC (GNU Compiller Collection) e os experimentos foram realizados em uma máquina com $8 \mathrm{~GB}$ de memória RAM, dois processadores de $2.6 \mathrm{GHz}$ Intel Xeon, com 6 núcleos cada e tecnologia Hyper Threading.

Geramos 20 instâncias do problema de corte em dois estágios com sobras aproveitáveis. Na Tabela 3.2 temos, para cada instância, o número que identifica a instância, a quantidade de itens, a quantidade de tipos distintos de itens, a quantidade de objetos e a quantidade de tipos distintos de objetos. O custo dos objetos não é informado explicitamente, mas consiste na sua área. Abaixo das quantidades temos as dimensões dos diferentes tipos de itens ou objetos no formato $N(W \times H)$, tal que $N$ refere-se a quantidade itens ou objetos daquele tipo. Instâncias com muitos tipos têm sua descrição identificada como nome de um conjunto. Abaixo segue a descrição de todos eles:

- $M_{6}=\{1(19 \times 21\}, 1(7 \times 20), 1(4 \times 20), 1(15 \times 20) ,1(14 \times 20), 1(14 \times 19), 1(17 \times 19)$, $1(10 \times 17), 1(13 \times 17), 1(5 \times 17), 1(16 \times 17), 1(20 \times 16), 1(5 \times 16), 1(3 \times 15), 1(5 \times 14)$, $1(18 \times 14), 1(10 \times 13), 1(14 \times 12), 1(11 \times 11), 1(2 \times 10), 1(7 \times 9), 1(14 \times 8), 1(13 \times 8)$, $1(9 \times 7), 1(7 \times 7), 1(16 \times 6), 1(20 \times 5), 1(2 \times 5), 1(9 \times 3), 1(17 \times 3), 1(5 \times 3), 1(4 \times 2)\}$, $P_{6}=\{3(52 \times 53)\}$;

- $M_{7}=\{8(11 \times 7), 5(7 \times 5), 9(9 \times 5), 5(10 \times 5) 10(3 \times 2)\}, P_{7}=\{5(25 \times 21), 5(27 \times 19)$, $3(30 \times 24)\}$;

- $M_{8}=\{1(22 \times 23), 1(17 \times 23), 1(13 \times 22), 1(7 \times 22), 1(9 \times 21), 1(5 \times 20), 1(20 \times 20)$, $1(6 \times 19), 1(7 \times 17), 1(14 \times 16), 1(12 \times 14), 1(15 \times 14), 1(20 \times 14), 1(9 \times 10), 1(16 \times 9)$, $1(20 \times 9), 1(18 \times 8), 1(3 \times 8), 1(12 \times 7), 1(18 \times 6), 1(20 \times 4), 1(9 \times 3), 1(6 \times 3)\}, P_{8}=$ $\{2(63 \times 60)\}$;

- $M_{10}=\{1(3 \times 5), 1(2 \times 5), 1(4 \times 3), 1(6 \times 3), 1(3 \times 3), 1(7 \times 3), 1(2 \times 2), 1(6 \times 2)$, $1(9 \times 2), 1(4 \times 2), 1(1 \times 2), 1(7 \times 2), 1(4 \times 1), 1(6 \times 1), 1(2 \times 1), 1(9 \times 1), 1(7 \times 1)\}$, $P_{10}=\{1(24 \times 14), 2(18 \times 10), 1(24 \times 13), 3(13 \times 10)\}$;

- $M_{11}=\{1(3 \times 5), 1(1 \times 5), 1(7 \times 5), 1(2 \times 5), 1(3 \times 4), 1(7 \times 4), 1(4 \times 4), 1(4 \times 3)$ $1(1 \times 3), 1(6 \times 3), 1(8 \times 3), 1(3 \times 3), 1(7 \times 3), 1(5 \times 2), 1(6 \times 2), 1(2 \times 2), 1(9 \times 2)$, $1(4 \times 2), 1(1 \times 2), 1(7 \times 2), 1(8 \times 1), 1(2 \times 1), 1(6 \times 1), 1(7 \times 1), 1(9 \times 1), 1(4 \times 1)$, $1(5 \times 1)\}, P_{11}=\{2(24 \times 14), 3(18 \times 10), 2(24 \times 13), 4(13 \times 10)\}$;

- $M_{12}=\{1(3 \times 5), 1(1 \times 5), 1(7 \times 5), 1(2 \times 5), 1(8 \times 5), 1(6 \times 4), 1(5 \times 4), 1(3 \times 4)$, $1(7 \times 4), 1(4 \times 4), 1(1 \times 4), 1(2 \times 4), 1(4 \times 3), 1(5 \times 3), 1(1 \times 3), 1(2 \times 3), 1(6 \times 3)$, $1(8 \times 3), 1(3 \times 3), 1(7 \times 3), 1(6 \times 2), 1(9 \times 2), 1(7 \times 2), 1(4 \times 2), 1(5 \times 2), 1(1 \times 2)$, $1(2 \times 2), 1(3 \times 2), 1(6 \times 1), 1(2 \times 1), 1(4 \times 1), 1(8 \times 1), 1(5 \times 1), 1(9 \times 1), 1(3 \times 1)$, $1(1 \times 1), 1(7 \times 1)\}, P_{12}=\{2(24 \times 14), 5(18 \times 10), 3(24 \times 13), 6(13 \times 10)\}$;

- $M_{13}=\{1(3 \times 5), 1(1 \times 5), 1(7 \times 5), 1(2 \times 5), 1(8 \times 5), 1(6 \times 4), 1(3 \times 4), 1(7 \times 4)$, $1(4 \times 4), 1(1 \times 4), 1(2 \times 4), 1(4 \times 3), 1(5 \times 3), 1(1 \times 3), 1(6 \times 3), 1(8 \times 3), 1(3 \times 3)$, $1(7 \times 3), 1(6 \times 2), 1(7 \times 2), 1(9 \times 2), 1(5 \times 2), 1(4 \times 2), 1(2 \times 2), 1(1 \times 2), 1(3 \times 2)$, $1(9 \times 1), 1(7 \times 1), 1(8 \times 1), 1(5 \times 1), 1(2 \times 1), 1(6 \times 1), 1(4 \times 1), 1(1 \times 1)\}$, $P_{13}=\{2(24 \times 14), 4(18 \times 10), 2(24 \times 13)\}$;

\footnotetext{
${ }^{2}$ Com o valor padrão de precisão relativa, o CPLEX declara como solução de algumas instâncias, pontos viáveis que não correspondem à solução esperada do problema. Como exemplo podemos citar a instância 3, cuja solução esperada contém uma sobra aproveitável com área 18, porém, com o valor padrão de precisão relativa, o CPLEX declara como solução um padrão de corte sem sobra aproveitável.
} 
- $M_{14}=\{2(2 \times 2), 1(6 \times 2), 1(4 \times 2), 1(1 \times 2), 2(7 \times 1), 1(4 \times 1), 1(9 \times 1), 1(6 \times 1)$, $1(2 \times 1)\}, P_{14}=\{1(24 \times 14), 1(18 \times 10), 1(24 \times 13)\}$;

- $M_{15}=\{1(1 \times 10), 1(6 \times 10), 1(4 \times 9), 1(8 \times 9), 1(9 \times 9), 1(5 \times 8), 1(2 \times 7), 1(4 \times 6)$, $1(10 \times 6), 1(6 \times 6), 1(10 \times 5), 1(5 \times 5), 1(8 \times 5), 1(4 \times 4), 1(7 \times 4), 1(10 \times 4), 1(6 \times 4)$, $1(7 \times 3), 1(10 \times 2)\}, P_{15}=\{3(28 \times 17), 3(16 \times 27), 4(13 \times 23)\}$;

- $M_{16}=\{1(2 \times 9), 1(2 \times 8), 1(5 \times 8), 1(3 \times 8), 1(4 \times 7), 1(5 \times 7), 1(4 \times 6), 1(6 \times 4)$, $1(2 \times 4), 1(3 \times 4), 1(4 \times 3), 1(2 \times 3), 1(6 \times 2), 1(5 \times 2), 1(1 \times 1), 1(2 \times 1), 1(3 \times 1)\}$, $P_{16}=\{3(19 \times 10), 1(19 \times 26)\}$;

- $M_{17}=\{2(63 \times 59), 1(63 \times 55), 1(48 \times 48), 1(17 \times 43), 1(98 \times 40), 1(38 \times 35), 2(114 \times 33)$, $1(24 \times 23), 1(62 \times 19), 2(110 \times 11)\}, P_{17}=\{2(290 \times 106), 2(148 \times 183), 2(194 \times 132)\}$.

\begin{tabular}{|c|c|c|c|c|}
\hline \multicolumn{5}{|c|}{ Descrição das Instâncias } \\
\hline \multirow{2}{*}{ \# } & \multicolumn{2}{|c|}{ Itens } & \multicolumn{2}{|c|}{ Objetos } \\
\hline & Qtde. Total & Qtde. Tipos & Qtde. Total & Qtde. Tipos \\
\hline \multirow{2}{*}{1} & 12 & 1 & 1 & 1 \\
\hline & \multicolumn{2}{|c|}{$12(2 \times 4)$} & \multicolumn{2}{|c|}{$1(14 \times 19)$} \\
\hline \multirow[b]{2}{*}{2} & 32 & 2 & 7 & 2 \\
\hline & \multicolumn{2}{|c|}{$\begin{array}{l}15(4 \times 7) \\
17(1 \times 4)\end{array}$} & \multicolumn{2}{|c|}{$\begin{array}{l}2(21 \times 24) \\
5(10 \times 18)\end{array}$} \\
\hline \multirow[b]{2}{*}{3} & 28 & 3 & 4 & 3 \\
\hline & \multicolumn{2}{|c|}{$\begin{array}{c}12(7 \times 1) \\
7(6 \times 1) \\
9(4 \times 1)\end{array}$} & \multicolumn{2}{|c|}{$\begin{array}{l}1(24 \times 14) \\
2(18 \times 10) \\
1(24 \times 13)\end{array}$} \\
\hline \multirow[b]{2}{*}{4} & 34 & 3 & 12 & 3 \\
\hline & \multicolumn{2}{|c|}{$\begin{array}{c}13(7 \times 6) \\
10(9 \times 4) \\
11(11 \times 3)\end{array}$} & \multicolumn{2}{|c|}{$\begin{array}{l}4(26 \times 19) \\
4(22 \times 23) \\
4(30 \times 17)\end{array}$} \\
\hline \multirow[b]{2}{*}{5} & 21 & 4 & 19 & 3 \\
\hline & \multicolumn{2}{|c|}{$\begin{array}{c}10(8 \times 10) \\
9(10 \times 3) \\
2(11 \times 2)\end{array}$} & \multicolumn{2}{|c|}{$\begin{array}{l}6(30 \times 11) \\
6(27 \times 13) \\
7(12 \times 26)\end{array}$} \\
\hline \multirow[b]{2}{*}{6} & 32 & 32 & 3 & 1 \\
\hline & \multicolumn{2}{|c|}{$\mathrm{M}_{6}$} & \multicolumn{2}{|c|}{$\mathrm{P}_{6}$} \\
\hline \multirow[b]{2}{*}{7} & 37 & 5 & 13 & 3 \\
\hline & \multicolumn{2}{|c|}{$\mathbf{M}_{7}$} & \multicolumn{2}{|c|}{$\mathrm{P}_{7}$} \\
\hline \multirow[b]{2}{*}{8} & 23 & 23 & 2 & 1 \\
\hline & \multicolumn{2}{|c|}{$\mathrm{M}_{8}$} & \multicolumn{2}{|c|}{$\mathrm{P}_{8}$} \\
\hline \multirow[b]{2}{*}{9} & 17 & 2 & 1 & 1 \\
\hline & \multicolumn{2}{|c|}{$\begin{array}{c}7(2 \times 4) \\
10(1 \times 3)\end{array}$} & \multicolumn{2}{|c|}{$1(14 \times 19)$} \\
\hline \multirow[b]{2}{*}{10} & 17 & 17 & 7 & 4 \\
\hline & & & & \\
\hline & 27 & 27 & 11 & 4 \\
\hline 11 & & & & \\
\hline & 37 & 37 & 16 & 4 \\
\hline 12 & & 12 & & \\
\hline & 34 & 34 & 8 & 3 \\
\hline 13 & & & & \\
\hline
\end{tabular}


Tabela 3.2 - continuação da página anterior

\begin{tabular}{|c|c|c|c|c|}
\hline \multirow{2}{*}{ \# } & \multicolumn{2}{|c|}{ Itens } & \multicolumn{2}{|c|}{ Objetos } \\
\hline & Qtde. Total & Qtde. Tipos & Qtde. Tota & Qtde. Tipos \\
\hline \multirow[b]{2}{*}{14} & 11 & 9 & 3 & 3 \\
\hline & \multicolumn{2}{|c|}{$\mathrm{M}_{14}$} & \multicolumn{2}{|c|}{$\mathrm{P}_{14}$} \\
\hline \multirow[b]{2}{*}{15} & 19 & 19 & 10 & 3 \\
\hline & \multicolumn{2}{|c|}{$\mathbf{M}_{15}$} & \multicolumn{2}{|c|}{$\mathrm{P}_{15}$} \\
\hline \multirow[b]{2}{*}{16} & 17 & 17 & 4 & 2 \\
\hline & \multicolumn{2}{|c|}{$\mathrm{M}_{16}$} & \multicolumn{2}{|c|}{$\mathrm{P}_{16}$} \\
\hline \multirow[b]{2}{*}{17} & 13 & 10 & 6 & 3 \\
\hline & \multicolumn{2}{|c|}{$\mathrm{M}_{17}$} & \multicolumn{2}{|c|}{$\mathrm{P}_{17}$} \\
\hline \multirow[b]{2}{*}{18} & 24 & 2 & 5 & 2 \\
\hline & \multicolumn{2}{|c|}{$\begin{array}{c}14(2 \times 11) \\
10(5 \times 5)\end{array}$} & \multicolumn{2}{|c|}{$\begin{array}{l}3(22 \times 17) \\
2(14 \times 30)\end{array}$} \\
\hline \multirow[b]{2}{*}{19} & 41 & 3 & 16 & 3 \\
\hline & \multicolumn{2}{|c|}{$\begin{array}{c}15(9 \times 7) \\
11(11 \times 6) \\
15(1 \times 5)\end{array}$} & \multicolumn{2}{|c|}{$\begin{array}{l}4(30 \times 22) \\
4(30 \times 24) \\
8(10 \times 21)\end{array}$} \\
\hline \multirow[b]{2}{*}{20} & 21 & 3 & 9 & 2 \\
\hline & \multicolumn{2}{|c|}{$\begin{array}{c}8(2 \times 11) \\
7(8 \times 9) \\
6(5 \times 5)\end{array}$} & \multicolumn{2}{|c|}{$\begin{array}{l}5(22 \times 17) \\
4(14 \times 30)\end{array}$} \\
\hline
\end{tabular}

Tabela 3.2: Descrição das instâncias $1,2, \ldots, 20$. Temos, para cada instância, o número que identifica a instância, a quantidade de itens, a quantidade de tipos distintos de itens, a quantidade de objetos e a quantidade de tipos distintos de objetos. Abaixo das quantidades temos as dimensões dos diferentes tipos de itens ou objetos no formato $N(W \times H)$, tal que $N$ refere-se a quantidade itens ou objetos daquele tipo. Instâncias com muitos tipos têm sua descrição identificada como nome de um conjunto descrito no texto.

A Tabela 3.3 ilustra para cada instância a quantidade de variáveis e restrições de cada modelo. A Tabela 3.4 ilustra os resultados dos experimentos numéricos com as 20 instâncias considerando os modelos $\mathcal{M}_{1}^{D E}$ e $\mathcal{M}_{3}^{D E}$. Para cada instância e cada modelo informamos na coluna "Lim. Inf.", o valor do limitante inferior obtido, na "Lim. Sup.", o valor do limitante superior obtido, na coluna "Valor das Sobras" o valor das sobras aproveitáveis considerando-se a melhor solução encontrada, na "Objetos Usados", a quantidade de objetos utilizados na solução, na "Iterações MIP", a quantidade de iterações MIP realizada pelo CPLEX, na "Nós B\&B", a quantidade de nós da árvore Branch \& Bound e na "Tempo" a quantidade de tempo de cpu em segundos que levou até a solução ser obtida (ou, caso ela não tenha sido obtida dentro do limite de tempo, exibe o valor deste, que é de 10800 segundos). Em todos os experimentos consideramos $M_{l}^{A}=W_{l} H_{l}, M_{l}^{W}=W_{l}$ e $M_{l}^{H}=H_{l}$ para $l=1, \ldots, p$. Podemos observar que os modelos não tiveram um desempenho homogêneo. O modelo $\mathcal{M}_{1}^{D E}$ teve desempenho melhor com as instâncias 10 e 15 e o modelo $\mathcal{M}_{3}^{D E}$ apresentou desempenho melhor nas demais. É importante notar que, considerando-se as instâncias $6,8,10,11,12,13,14,15$ e 16 , o modelo $\mathcal{M}_{3}^{D E}$ apresenta uma quantidade maior de variáveis (binárias e inteiras) e restrições que o modelo $\mathcal{M}_{1}^{D E}$. Observando as instâncias não resolvidas por cada modelo, vemos que o modelo $\mathcal{M}_{3}^{D E}$ termina com limitantes melhores que os obtidos por $\mathcal{M}_{1}^{D E}$ nas instâncias 4, 12 e 19 e resolve todas as demais não resolvidas por $\mathcal{M}_{1}^{D E}$. Assim é possível afirmar que, de uma forma geral, o modelo $\mathcal{M}_{3}^{D E}$ obteve um desempenho computacional melhor que o modelo $\mathcal{M}_{1}^{D E}$. Nas Figuras 3.5 - 3.21 é possível ver ilustrações das soluções encontradas pelo modelo $\mathcal{M}_{3}^{D E}$. Em cada objeto, a área hachurada representa a sobra aproveitável (quando presente), os espaços em branco correspondem ao desperdício e os retângulos coloridos correspondem aos 
itens.

A Tabela 3.5 ilustra alguns experimentos com o modelo $\mathcal{M}_{3}^{D E}$, sem a restrição (3.52). Comparando os valores desta tabela com os da Tabela 3.4 podemos notar que doze instâncias necessitaram da realização de mais iterações MIP e da exploração de mais nós no caso em que a restrição (3.52) não foi incluída, são elas as instâncias 3, 7, 8, 9, 10, 11, 13, 14, 16, 18 e 20. Por outro lado, seis instâncias foram resolvidas necessitando de menos iterações MIP e da exploração de menos nós no caso em que a restrição (3.52) não foi incluída, são elas as instâncias: 2, 5, 6, 15, 17 e 19. Vale notar que sem a restrição (3.52) o CPLEX foi capaz de resolver a instância 19, que não é resolvida quando consideramos a restrição (3.52), mas não foi capaz de obter solução para a instância 7 (que é resolvida quando consideramos a restrição (3.52)). Notamos também que considerando a instância 4, obtivemos um ( $\mathrm{gap}$ ) de integralidade menor ao incluirmos a restrição (3.52) e no caso da instância 12, obtivemos um (gap) de integralidade menor ao removermos a mesma restrição.

A Figura 3.4 ilustra a evolução dos limitantes encontrados pelo CPLEX ao longo da resolução das instâncias 2, 6, 12 e 15 com os dois modelos considerados. Consideramos essas instâncias pois ilustram casos em que o modelo $\mathcal{M}_{1}^{D E}$ tem melhor desempenho (instância 15), em que modelo $\mathcal{M}_{3}^{D E}$ tem melhor desempenho (instância 6), em que o modelo $\mathcal{M}_{1}^{D E}$ não resolve o problema em 3 horas (instância 2) e em que nenhum dos modelos terminam em 3 horas (instância 12). Em todos os casos podemos observar que o limitante superior muda poucas vezes ao longo da execução de cada caso e que boa parte do esforço computacional está concentrado em obter limitantes inferiores melhores. Uma forma de melhorar ambos os modelos seria acrescentar restrições que permitissem obter melhores limitantes inferiores ao resolver os subproblemas com as restrições de integralidade relaxadas.

\begin{tabular}{|c||c|c|c|c||c|c|c|c|}
\hline \multicolumn{1}{|c||}{} & \multicolumn{9}{c||}{ Modelo $\mathcal{M}_{1}^{D E}$} & \multicolumn{4}{c|}{ Modelo $\mathcal{M}_{3}^{D E}$} \\
\hline \multirow{2}{*}{ Instância } & $\begin{array}{c}\text { Variáveis } \\
\text { continuas }\end{array}$ & $\begin{array}{c}\text { Variáveis } \\
\text { binárias }\end{array}$ & $\begin{array}{c}\text { Variáveis } \\
\text { inteiras }\end{array}$ & Restrições & $\begin{array}{c}\text { Variáveis } \\
\text { continuas }\end{array}$ & $\begin{array}{c}\text { Variáveis } \\
\text { binárias }\end{array}$ & $\begin{array}{c}\text { Variáveis } \\
\text { inteiras }\end{array}$ & Restrições \\
\hline 1 & 2 & 80 & 0 & 95 & 2 & 14 & 12 & 65 \\
2 & 14 & 3710 & 0 & 3763 & 14 & 238 & 329 & 684 \\
3 & 8 & 1632 & 0 & 1672 & 8 & 120 & 236 & 452 \\
4 & 24 & 7164 & 0 & 7234 & 24 & 432 & 840 & 1410 \\
5 & 38 & 4426 & 0 & 4504 & 38 & 437 & 950 & 1507 \\
6 & 6 & 1590 & 0 & 1631 & 6 & 102 & 1584 & 1791 \\
7 & 26 & 9164 & 0 & 9240 & 26 & 507 & 1391 & 2048 \\
8 & 4 & 556 & 0 & 585 & 4 & 50 & 552 & 677 \\
9 & 2 & 154 & 0 & 174 & 2 & 19 & 24 & 97 \\
10 & 14 & 1084 & 0 & 1122 & 14 & 133 & 1071 & 1276 \\
11 & 22 & 4179 & 0 & 4239 & 22 & 319 & 4158 & 4591 \\
12 & 32 & 11280 & 0 & 11365 & 32 & 624 & 11248 & 12031 \\
13 & 16 & 4776 & 0 & 4834 & 16 & 288 & 4760 & 5174 \\
14 & 6 & 203 & 0 & 223 & 6 & 39 & 177 & 258 \\
15 & 20 & 1920 & 0 & 1969 & 20 & 210 & 1900 & 2197 \\
16 & 8 & 620 & 0 & 649 & 8 & 76 & 612 & 751 \\
17 & 12 & 558 & 0 & 589 & 12 & 90 & 420 & 567 \\
18 & 10 & 1510 & 0 & 1549 & 10 & 130 & 190 & 407 \\
19 & 32 & 13808 & 0 & 13897 & 32 & 688 & 1312 & 2171 \\
20 & 18 & 2096 & 0 & 2144 & 18 & 207 & 396 & 693 \\
\hline
\end{tabular}

Tabela 3.3: Quantidade de variáveis e restrições dos modelos $\mathcal{M}_{1}^{D E}$ e $\mathcal{M}_{3}^{D E}$ para cada umas das 20 instâncias consideradas. 

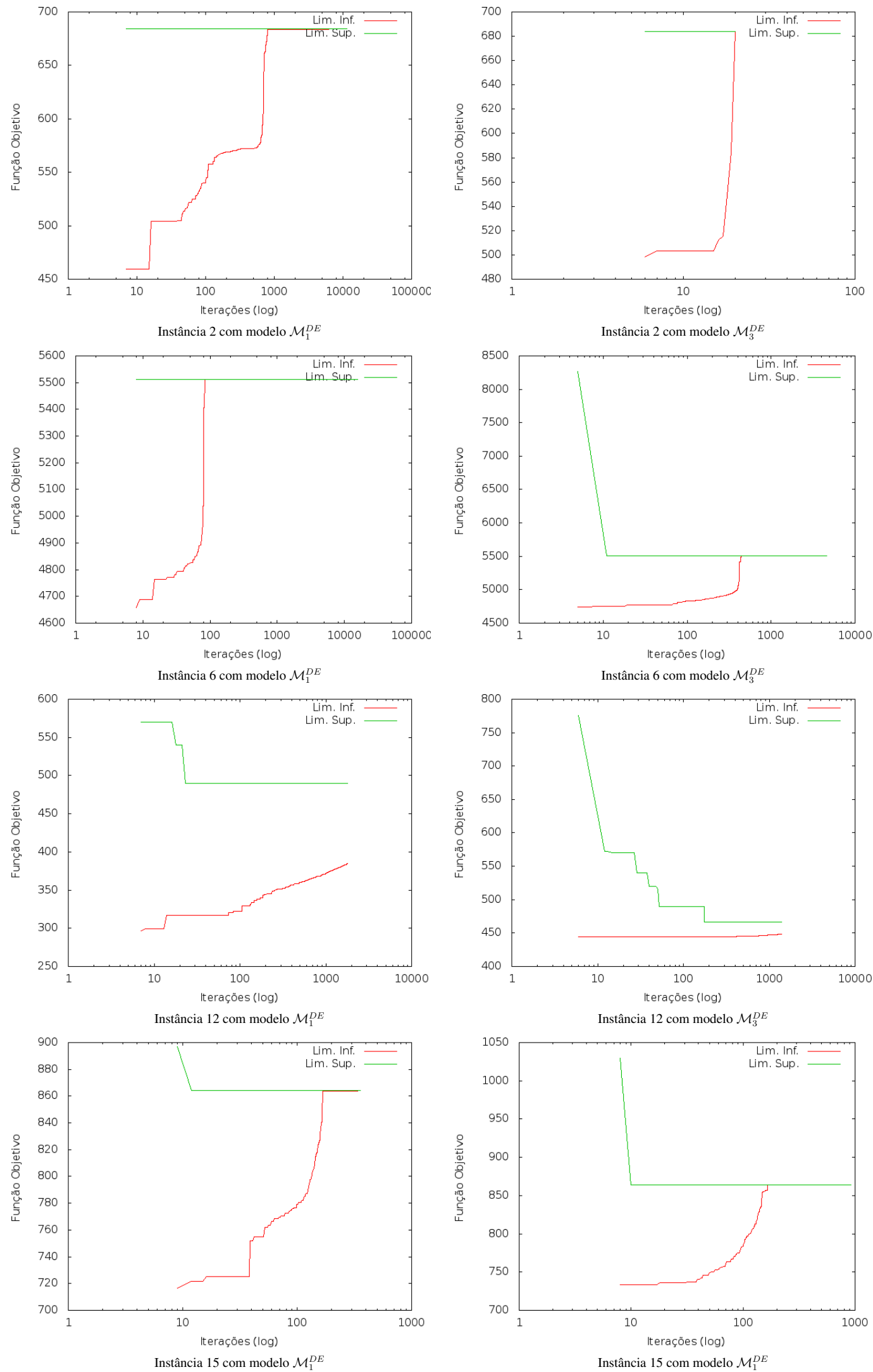

Figura 3.4: Evolução dos limitantes superiores e inferiores obtidos durante a execução de ambos os modelos considerando-se as instâncias 2, 6, 12 e 15. 


\begin{tabular}{|c|c|c|c|c|c|c|c|}
\hline \multicolumn{8}{|c|}{ Modelo $\mathcal{M}_{1}^{D E}$} \\
\hline \multirow[t]{2}{*}{ Instância } & \multicolumn{4}{|c|}{ Dados das soluções } & \multicolumn{3}{|c|}{ Medidas de desempenho } \\
\hline & Lim. Inf. & Lim. Sup. & Valor das Sobras & Objetos Usados & Iterações MIP & Nós $B \& B$ & Tempo (s) \\
\hline 1 & 265,421052631579 & 265,421052631579 & 40964 & 1 & 65 & 0 & 0,01 \\
\hline 2 & 683,960221852719 & 683,973135611433 & 18000 & - & - & - & $\geq 10800,00$ \\
\hline 3 & 179,988219895288 & 179,988219895288 & 3240 & 1 & 47802 & 1462 & 1,47 \\
\hline 4 & 1251,70595672538 & 1506,00000000000 & 0 & - & - & - & $\geq 10800,00$ \\
\hline 5 & 1159,67857142857 & 1364,99458441457 & 11232 & - & - & - & $\geq 10800,00$ \\
\hline 6 & 5511,93580292276 & 5511,93710691824 & 1433120 & - & - & - & $\geq 10800,00$ \\
\hline 7 & 1256,99023853794 & 1745,98983330862 & 43200 & - & - & - & $\geq 10800,00$ \\
\hline 8 & 7559,61591117216 & 7559,61666666667 & 10954440 & 2 & 3394531 & 157148 & 43,51 \\
\hline 9 & 265,368421052632 & 265,368421052632 & 44688 & 1 & 133 & 0 & 0,01 \\
\hline 10 & 259,979247252410 & 259,979247252410 & 6760 & 2 & 513157 & 15454 & 5,35 \\
\hline 11 & 359,999964035205 & 360,000000000000 & 0 & 2 & 64596297 & 506642 & 7527,93 \\
\hline 12 & 385,043448027794 & 489,995852661977 & 3240 & - & - & - & $\geq 10800,00$ \\
\hline 13 & 491,972726055643 & 491,972774808463 & 14976 & 2 & 68492224 & 384154 & 9405,41 \\
\hline 14 & 179,919881305638 & 179,919881305638 & 19440 & 1 & 402 & 0 & 0,04 \\
\hline 15 & 863,982605603292 & 863,982689750339 & 27648 & 2 & 4094147 & 68785 & 131,41 \\
\hline 16 & 380,000000000000 & 380,000000000000 & 0 & 2 & 7016 & 288 & 0,87 \\
\hline 17 & 51215,9236703395 & 51215,9287027284 & 332852784 & 2 & 47468 & 1432 & 1,20 \\
\hline 18 & 748,000000000000 & 748,000000000000 & 0 & 2 & 415352 & 11869 & 6,94 \\
\hline 19 & 1529,90176344911 & 2010,00000000000 & 0 & - & - & - & $\geq 10800,00$ \\
\hline 20 & 1167,96474730364 & 1167,96486213327 & 49368 & 3 & 130354931 & 2558999 & 3886,66 \\
\hline \multicolumn{8}{|c|}{ Modelo $\mathcal{M}_{3}^{D E}$} \\
\hline \multirow[t]{2}{*}{ Instância } & \multicolumn{4}{|c|}{ Dados das soluções } & \multicolumn{3}{|c|}{ Medidas de desempenho } \\
\hline & Lim. Inf. & Lim. Sup. & Valor das Sobras & Objetos Usados & Iterações MIP & Nós $B \& B$ & Tempo (s) \\
\hline 1 & 265,421052631579 & 265,421052631579 & 40964 & 1 & 18 & 0 & 0,00 \\
\hline 2 & 683,973135611433 & 683,973135611433 & 18000 & 2 & 47344 & 1977 & 0,99 \\
\hline 3 & 179,988219895288 & 179,988219895288 & 3240 & 1 & 1401 & 74 & 0,32 \\
\hline 4 & 1493,99577595597 & 1506,00000000000 & 0 & - & - & - & $\geq 10800,00$ \\
\hline 5 & 1364,99444810014 & 1364,99458441457 & 11232 & 4 & 4454864 & 140623 & 78,75 \\
\hline 6 & 5511,93655593184 & 5511,93710691824 & 1433120 & 2 & 38072670 & 1115032 & 1818,80 \\
\hline 7 & 1745,98965889728 & 1745,98983330862 & 43200 & 3 & 64571094 & 1239554 & 2577,75 \\
\hline 8 & 7559,61591430293 & 7559,61666666667 & 10954440 & 2 & 106190 & 5670 & 1,34 \\
\hline 9 & 265,368421052632 & 265,368421052632 & 44688 & 1 & 49 & 0 & 0,00 \\
\hline 10 & 259,979227435042 & 259,979247252410 & 6760 & 2 & 1219884 & 92199 & 15,64 \\
\hline 11 & 359,999965828321 & 360,000000000000 & 0 & 2 & 17672489 & 292336 & 871,52 \\
\hline 12 & 448,465753424657 & 466,000000000000 & 0 & - & - & - & $\geq 10800,00$ \\
\hline 13 & 491,972725959800 & 491,972774691129 & 14976 & 2 & 15356068 & 212033 & 793,86 \\
\hline 14 & 179,919881305638 & 179,919881305638 & 19440 & 1 & 282 & 0 & 0,02 \\
\hline 15 & 863,982603738071 & 863,982689750339 & 27648 & 2 & 7589442 & 192192 & 464,81 \\
\hline 16 & 380,000000000000 & 380,000000000000 & 0 & 2 & 7516 & 439 & 0,62 \\
\hline 17 & 51215,9238206946 & 51215,9287027284 & 332852784 & 2 & 29659 & 1583 & 0,82 \\
\hline 18 & 748,000000000000 & 748,000000000000 & 0 & 2 & 3950 & 457 & 0,42 \\
\hline 19 & 2009,98873449521 & 2010,00000000000 & 0 & - & - & - & $\geq 10800,00$ \\
\hline 20 & 1167,96486213327 & 1167,96486213327 & 49368 & 3 & 83275 & 3245 & 1,44 \\
\hline
\end{tabular}

Tabela 3.4: Desempenho do CPLEX na resolução das 20 instâncias dos modelos $\mathcal{M}_{1}^{D E}$ e $\mathcal{M}_{3}^{D E}$. 


\begin{tabular}{|c||cc|r|r|r|r|r|}
\hline \multicolumn{9}{|c|}{ Modelo $\mathcal{M}_{3}^{D E}$ desconsiderando a restrição (3.52) } \\
\hline \multirow{2}{*}{ Instância } & \multicolumn{9}{c|}{ Dados das soluções } & \multicolumn{3}{c|}{ Medidas de desempenho } \\
\cline { 2 - 8 } & Lim. Inf. & Lim. Sup. & Valor das Sobras & Objetos Usados & Iterações MIP & Nós B\&B & Tempo (s) \\
\hline 1 & 265,421052631523 & 265,421052631523 & 40964 & 1 & 34 & 0 & 0,00 \\
2 & 683,973135611433 & 683,973135611433 & 18000 & 2 & 35296 & 1202 & 0,99 \\
3 & 179,988219895288 & 179,988219895288 & 3240 & 1 & 5984 & 573 & 0,39 \\
4 & 1493,98759243139 & 1506,00000000000 & 0 & - & - & - & $\geq 10800,00$ \\
5 & 1364,99445075969 & 1364,99458441457 & 11232 & 4 & 3479089 & 111217 & 48,13 \\
6 & 5511,93655572517 & 5511,93710691824 & 1433120 & 2 & 14150577 & 602451 & 220,83 \\
7 & 1745,98186940038 & 1745,98983330862 & 43200 & - & - & - & $\geq 10800,00$ \\
8 & 7559,61591112905 & 7559,61666666667 & 10954440 & 2 & 170408 & 8027 & 1,70 \\
9 & 265,368421052632 & 265,368421052632 & 44688 & 1 & 53 & 0 & 0,00 \\
10 & 259,979220184183 & 259,979245817116 & 6760 & 2 & 1860968 & 140287 & 25,04 \\
11 & 359,999964009661 & 360,000000000000 & 0 & 2 & 19343745 & 311161 & 1069,97 \\
12 & 448,999999999999 & 466,000000000000 & - & - & - & $\geq 10800,00$ \\
13 & 491,972725852670 & 491,972774869110 & 14976 & 2 & 22319479 & 363425 & 1244,46 \\
14 & 179,919881305638 & 179,919881305638 & 19440 & 1 & 319 & 1 & 0,11 \\
15 & 863,982604801695 & 863,982689750339 & 27648 & 2 & 4216649 & 94544 & 126,03 \\
16 & 380,000000000000 & 380,000000000000 & 0 & 2 & 9340 & 480 & 0,68 \\
17 & 51215,9238636991 & 51215,9287027284 & 332852784 & 2 & 11717 & 548 & 0,73 \\
18 & 748,000000000000 & 748,000000000000 & 0 & 2 & 11238 & 955 & 0,39 \\
19 & 2010,00000000000 & 2010,00000000000 & 0 & 5 & 6816907 & 145479 & 132,76 \\
20 & 1167,96484427077 & 1167,96486213323 & 49368 & 3 & 136109 & 4919 & 1,72 \\
\hline
\end{tabular}

Tabela 3.5: Desempenho do CPLEX na resolução das 20 instâncias do modelo $\mathcal{M}_{3}^{D E}$ desconsiderando a restrição (3.52).

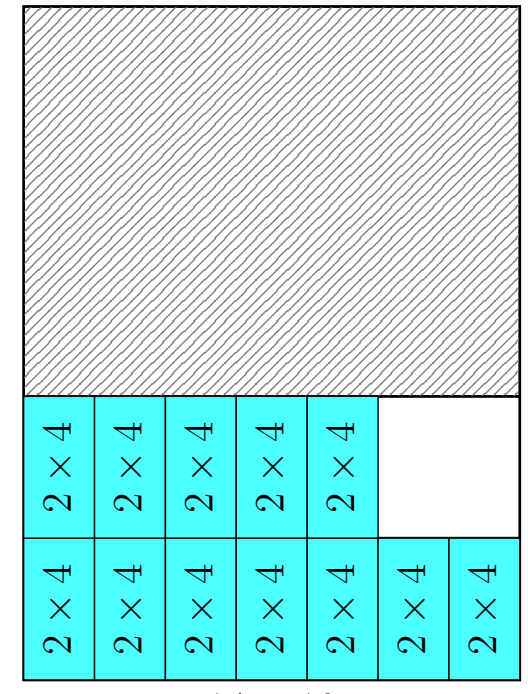

$14 \times 19$

Figura 3.5: Ilustração da solução da instância 1 do Modelo $\left(\mathcal{M}_{3}^{D E}\right)$ 


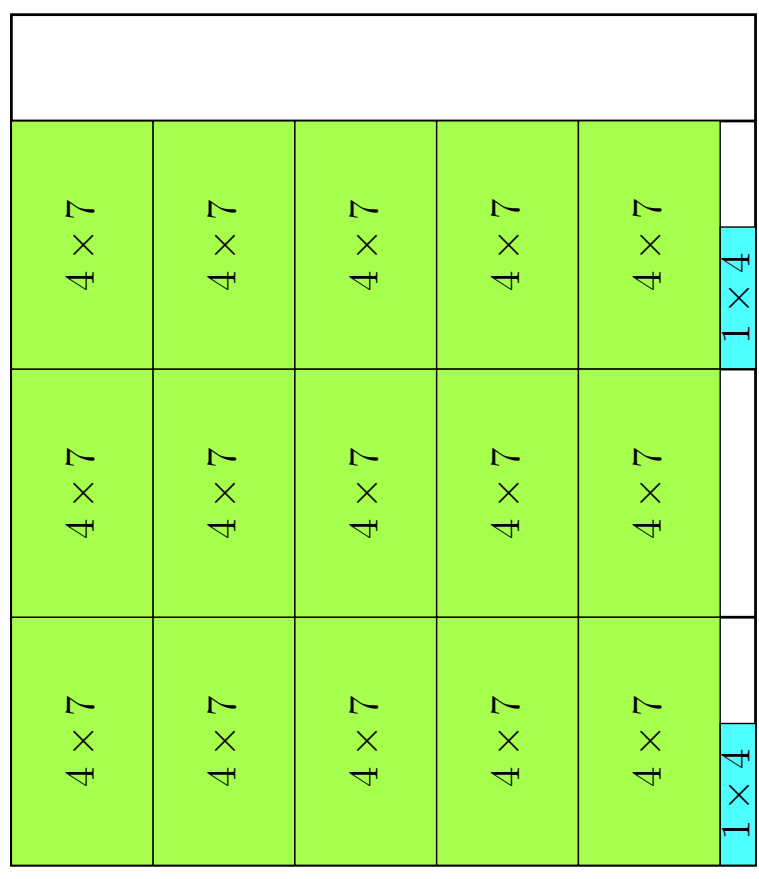

$21 \times 24$

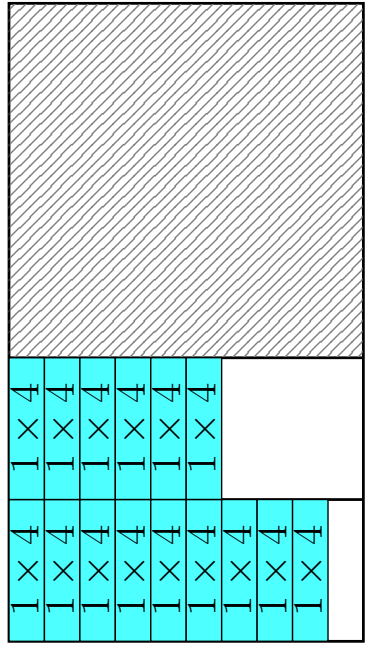

$10 \times 18$

Figura 3.6: Ilustração da solução da instância 2 do Modelo $\left(\mathcal{M}_{3}^{D E}\right)$

\begin{tabular}{|c|c|c|c|}
\hline $6 \times 1$ & \multicolumn{2}{|c|}{$6 \times 1$} & $\times 1$ \\
\hline $6 \times 1$ & \multicolumn{2}{|c|}{$6 \times 1$} & $\times 1$ \\
\hline $6 \times 1$ & $4 \times 1$ & $4 \times 1$ & $4 \times 1$ \\
\hline $7 \times 1$ & \multicolumn{2}{|c|}{$7 \times 1$} & $4 \times 1$ \\
\hline $7 \times 1$ & \multicolumn{2}{|c|}{$7 \times 1$} & $4 \times 1$ \\
\hline $7 \times 1$ & \multicolumn{2}{|c|}{$7 \times 1$} & $4 \times 1$ \\
\hline $7 \times 1$ & \multicolumn{2}{|c|}{$7 \times 1$} & $4 \times 1$ \\
\hline $7 \times 1$ & \multicolumn{2}{|c|}{$7 \times 1$} & $4 \times 1$ \\
\hline $7 \times 1$ & \multicolumn{2}{|c|}{$7 \times 1$} & $4 \times 1$ \\
\hline
\end{tabular}

$18 \times 10$

Figura 3.7: Ilustração da solução da instância 3 do Modelo $\left(\mathcal{M}_{3}^{D E}\right)$ 


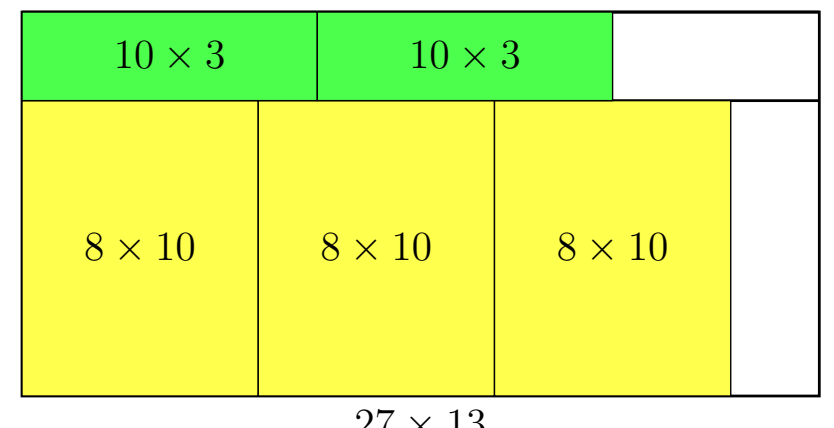

$27 \times 13$

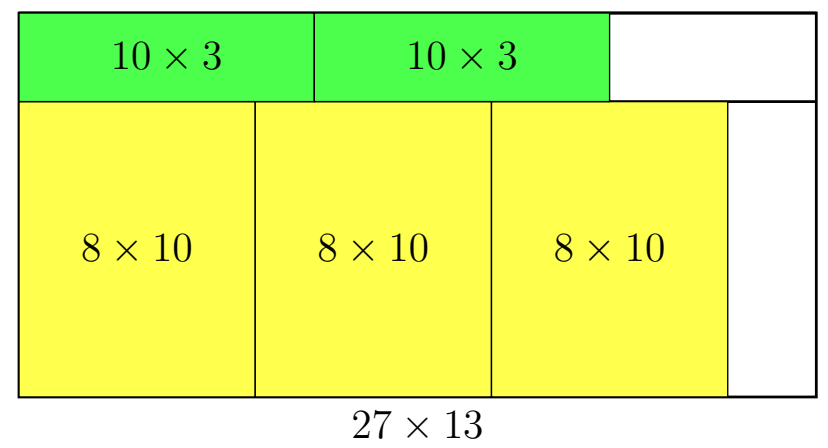

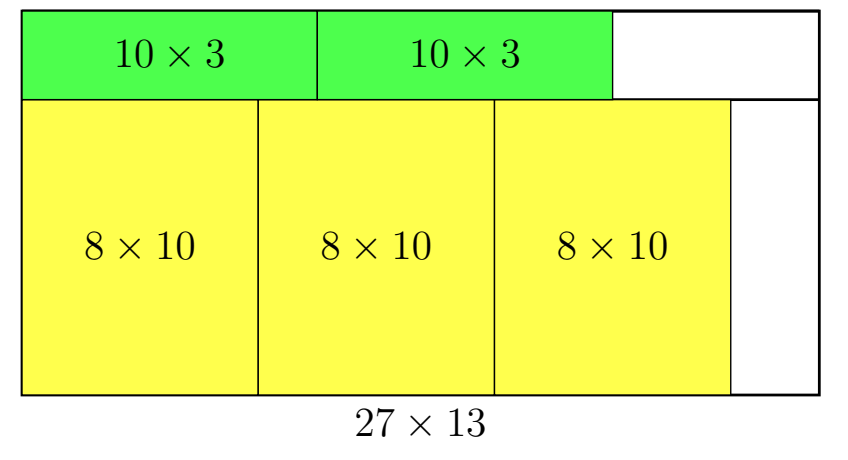

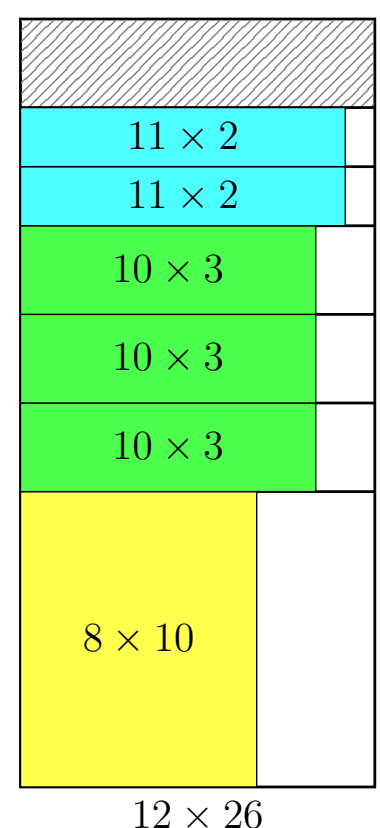

$12 \times 26$

Figura 3.8: Ilustração da solução da instância 5 do Modelo $\left(\mathcal{M}_{3}^{D E}\right)$ 

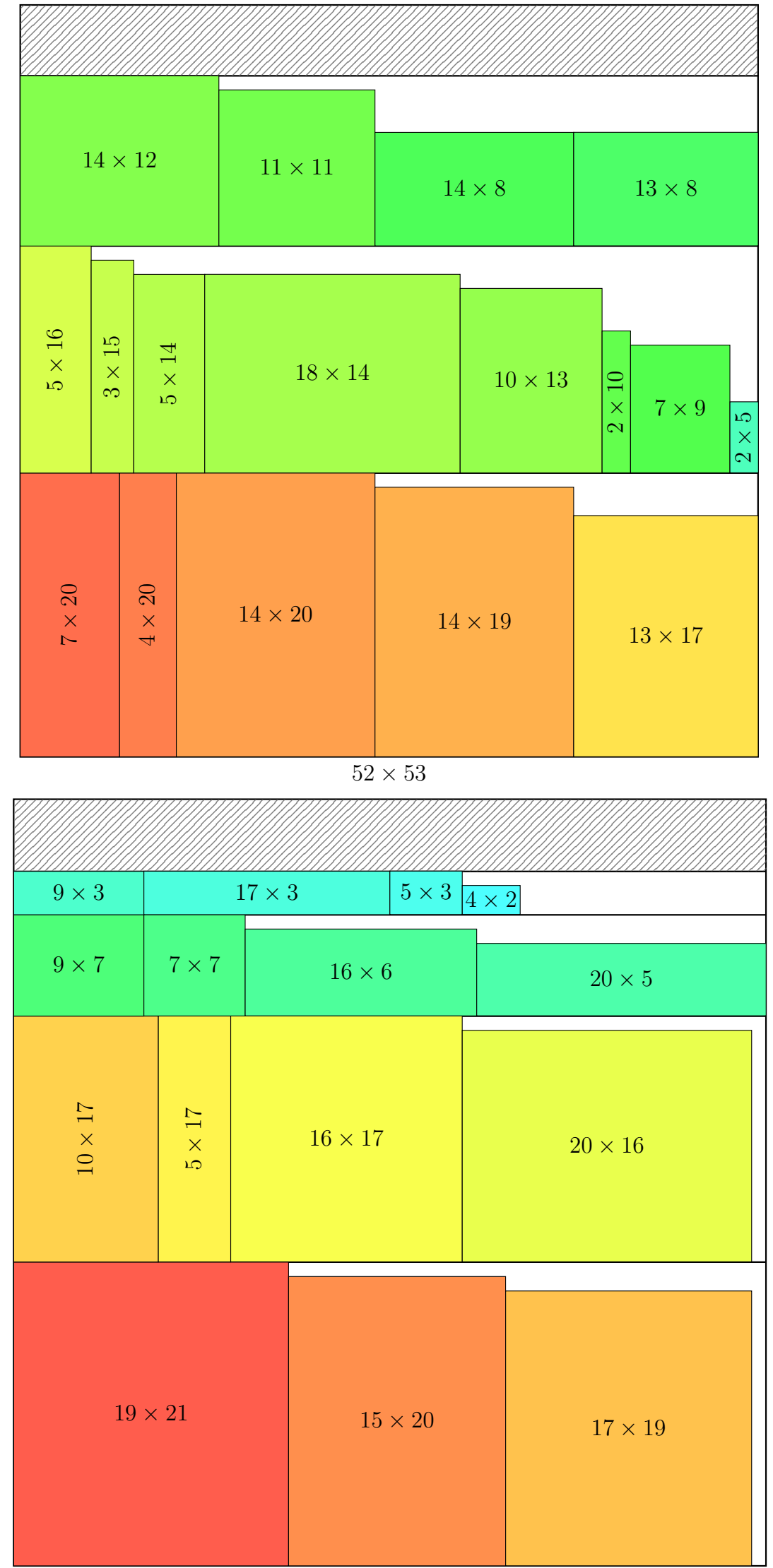

$52 \times 53$

Figura 3.9: Ilustração da solução da instância 6 do Modelo $\left(\mathcal{M}_{3}^{D E}\right)$ 


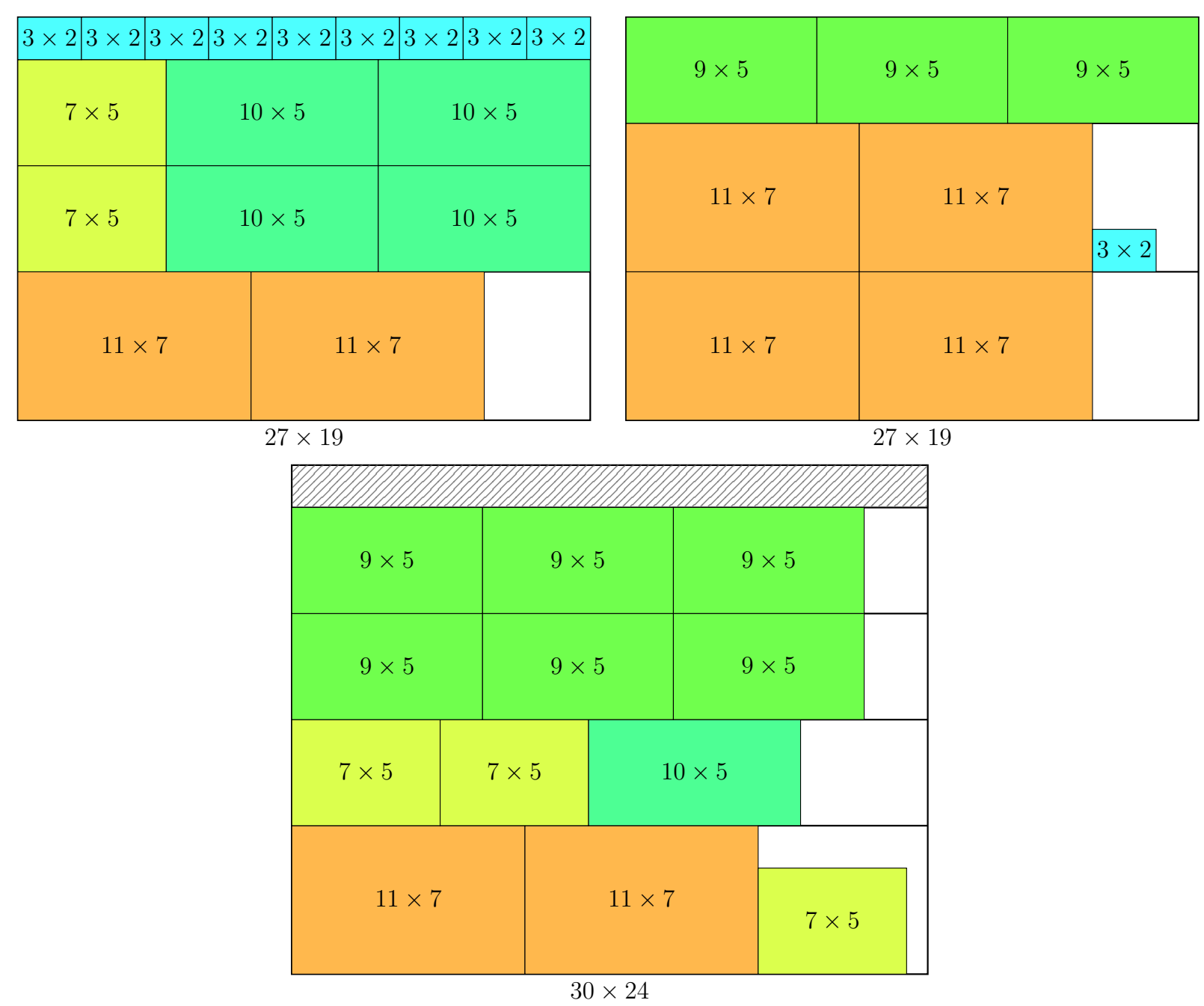

Figura 3.10: Ilustração da solução da instância 7 do Modelo $\left(\mathcal{M}_{3}^{D E}\right)$ 


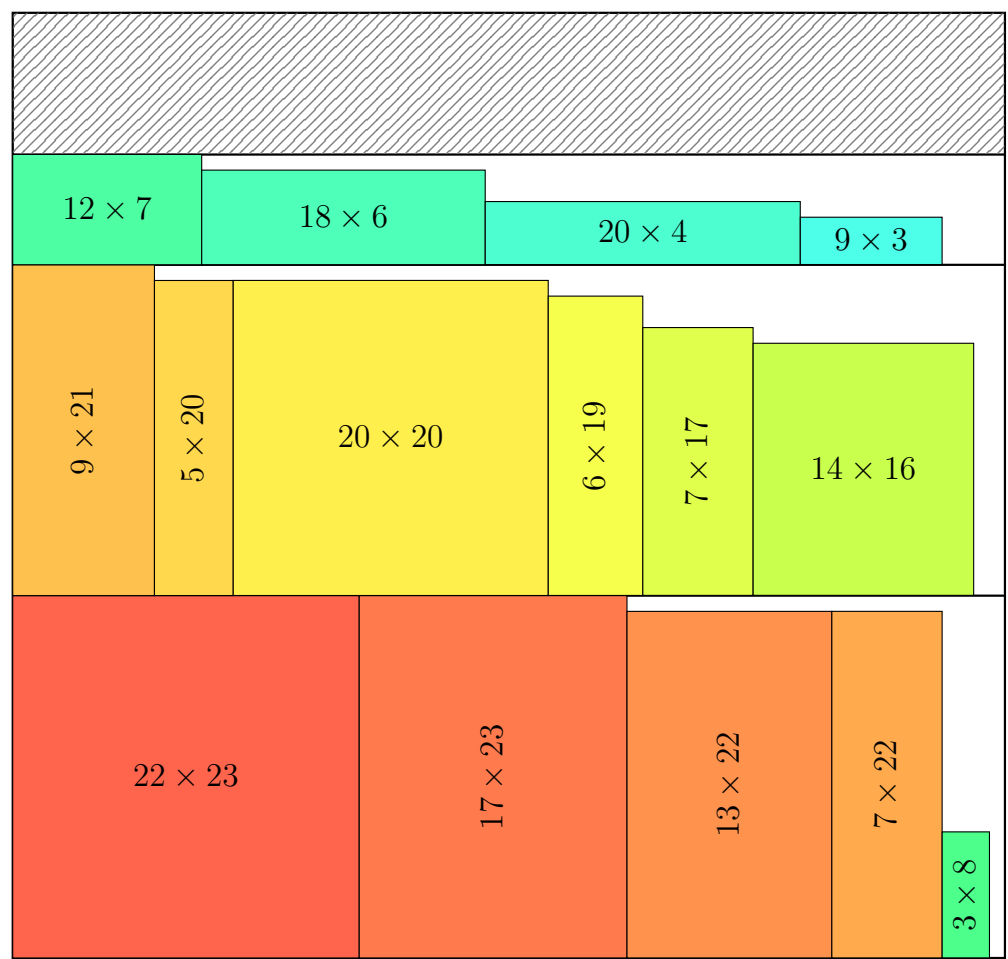

$63 \times 60$

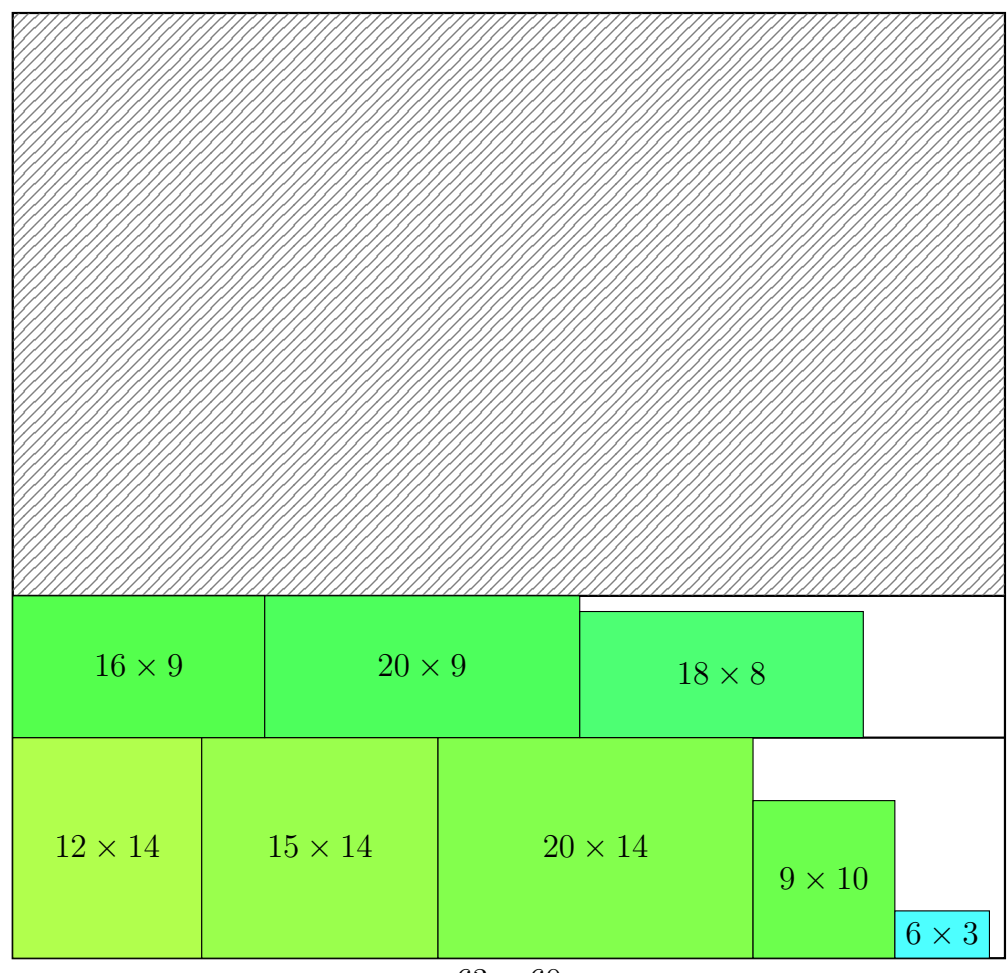

$63 \times 60$

Figura 3.11: Ilustração da solução da instância 8 do Modelo $\left(\mathcal{M}_{3}^{D E}\right)$ 


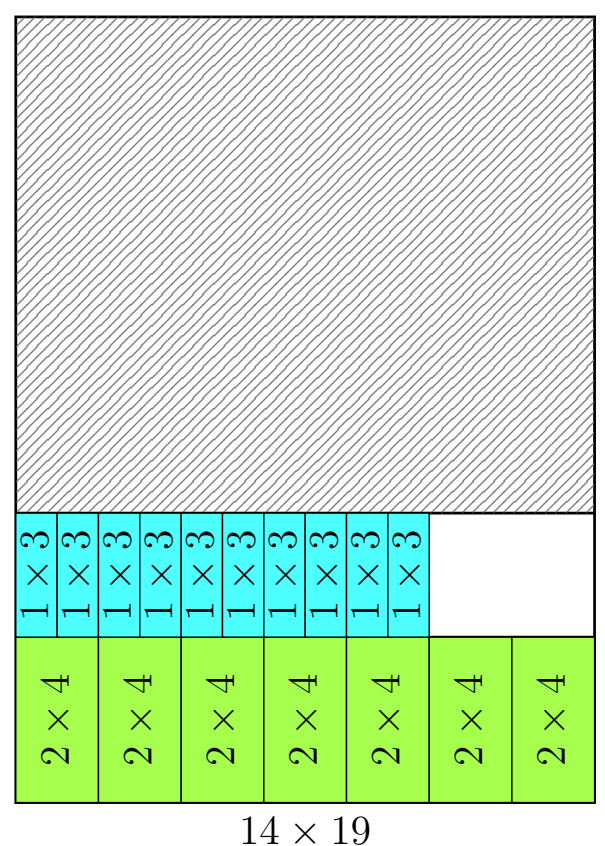

Figura 3.12: Ilustração da solução da instância 9 do Modelo $\left(\mathcal{M}_{3}^{D E}\right)$

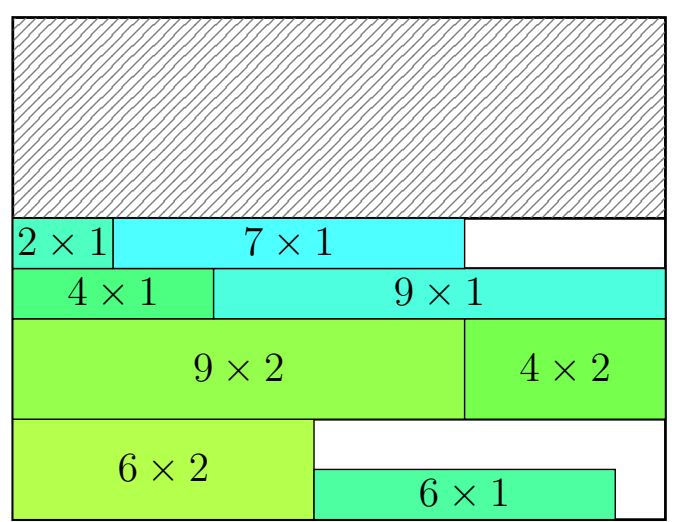

$13 \times 10$

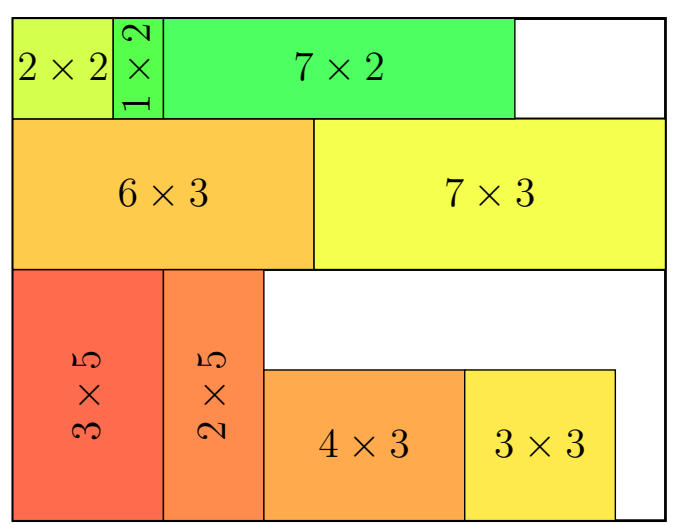

$13 \times 10$

Figura 3.13: Ilustração da solução da instância 10 do Modelo $\left(\mathcal{M}_{3}^{D E}\right)$ 


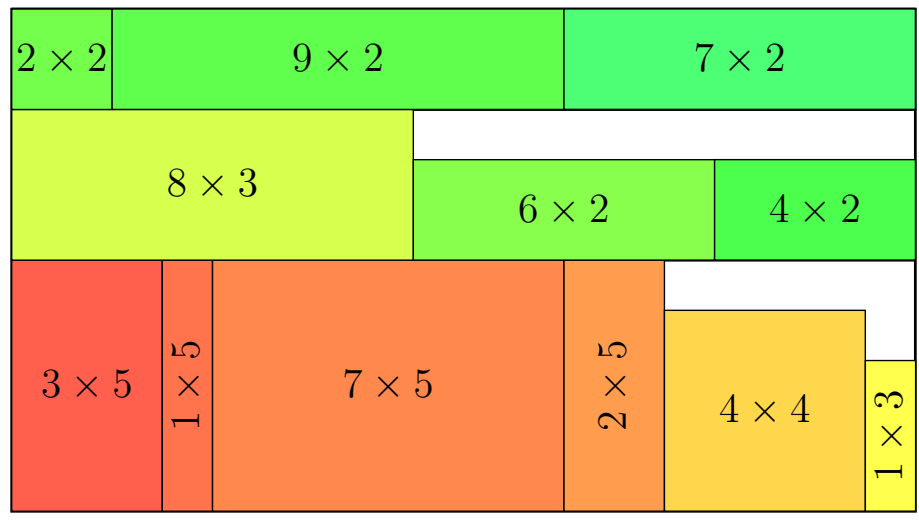

$18 \times 10$

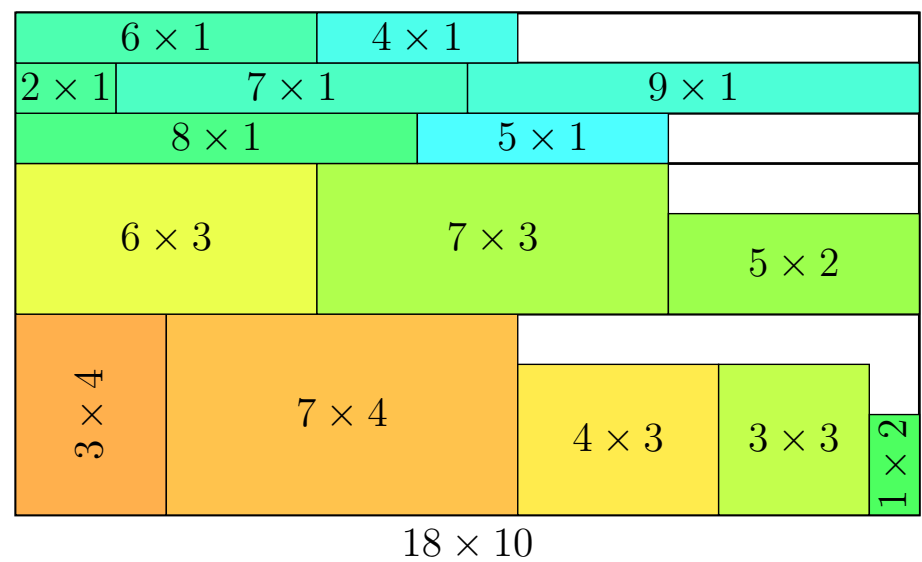

Figura 3.14: Ilustração da solução da instância 11 do Modelo $\left(\mathcal{M}_{3}^{D E}\right)$ 


\begin{tabular}{|c|c|c|c|c|}
\hline \multicolumn{2}{|c|}{$9 \times 2$} & \multicolumn{2}{|c|}{$5 \times 2$} & $4 \times$ \\
\hline $6 \times 2$ & & $7 \times 2$ & $\begin{array}{l}N \\
x\end{array}$ & $3 \times 2$ \\
\hline $6 \times 3$ & & $8 \times 3$ & & $3 \times 3$ \\
\hline $4 \times 3$ & $5 \times 3$ & & & \\
\hline
\end{tabular}

$18 \times 10$

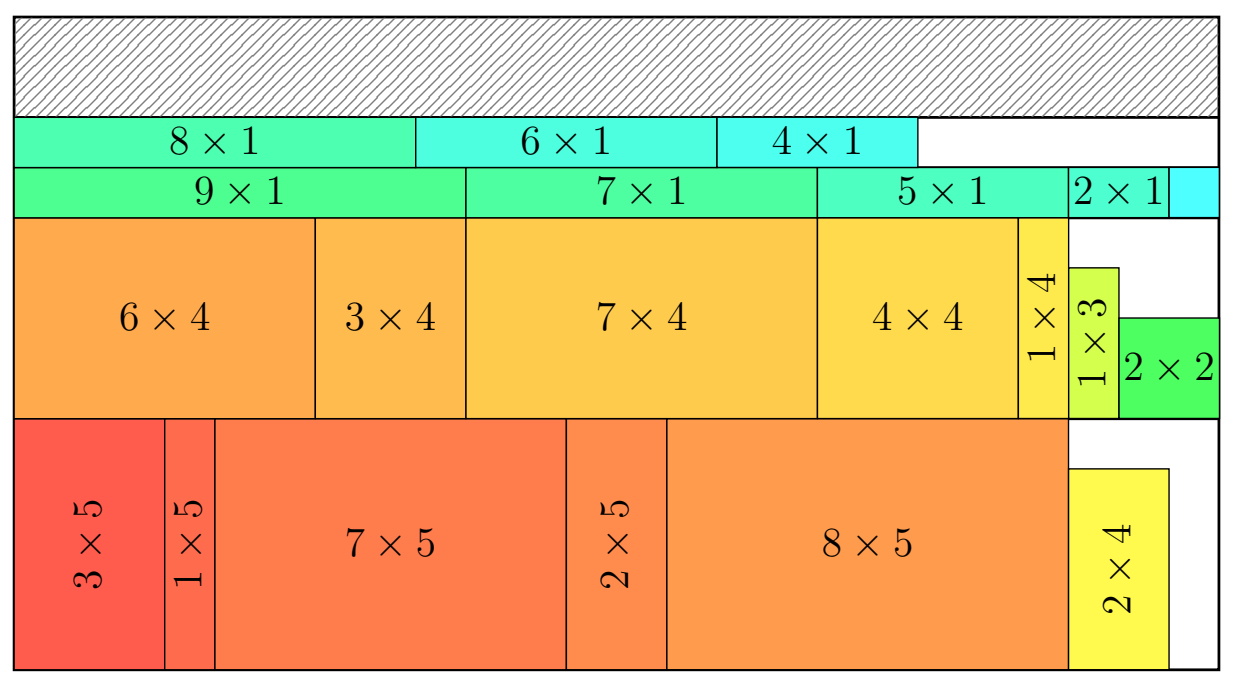

$24 \times 13$

Figura 3.15: Ilustração da solução da instância 13 do Modelo $\left(\mathcal{M}_{3}^{D E}\right)$

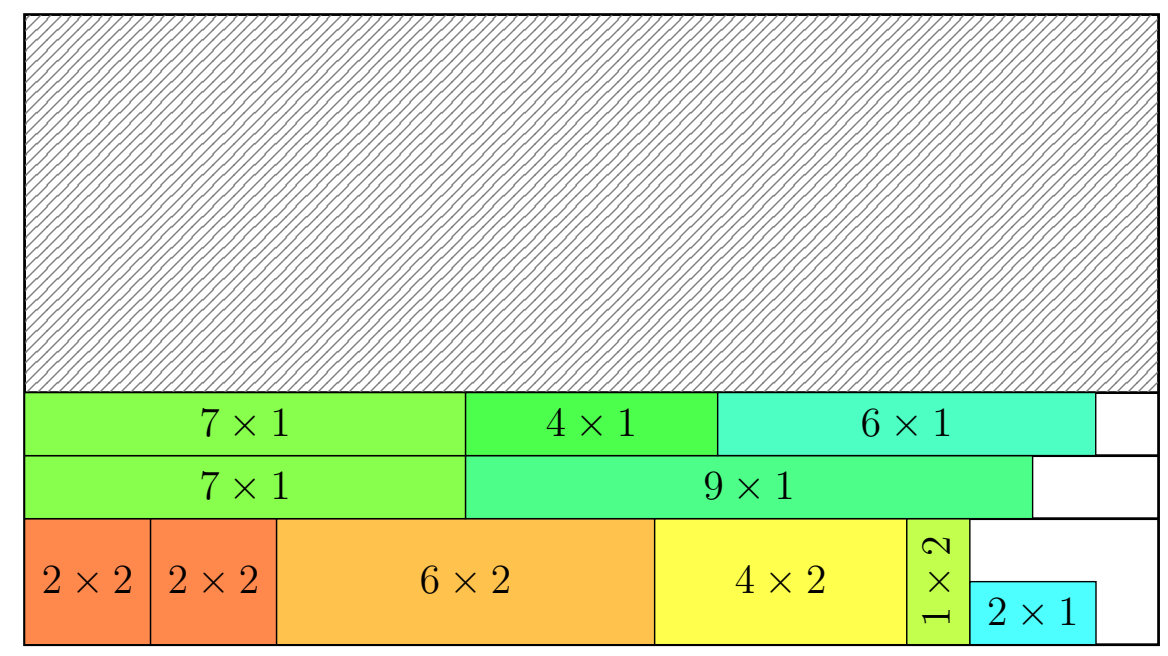

$18 \times 10$

Figura 3.16: Ilustração da solução da instância 14 do Modelo $\left(\mathcal{M}_{3}^{D E}\right)$ 


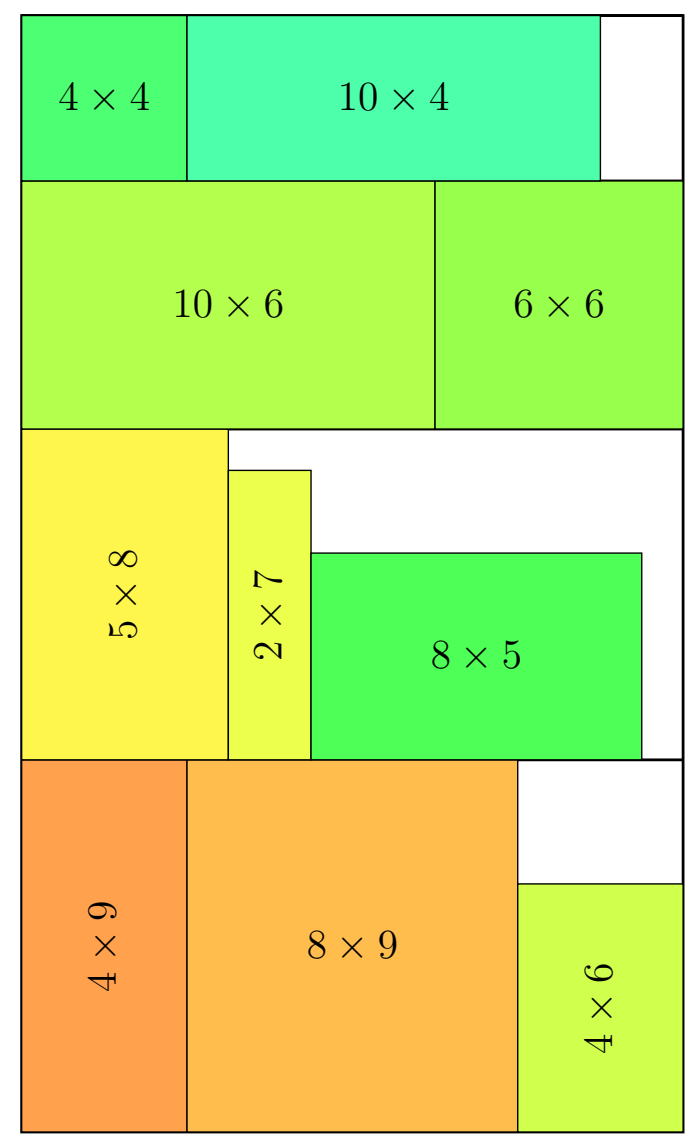

$16 \times 27$

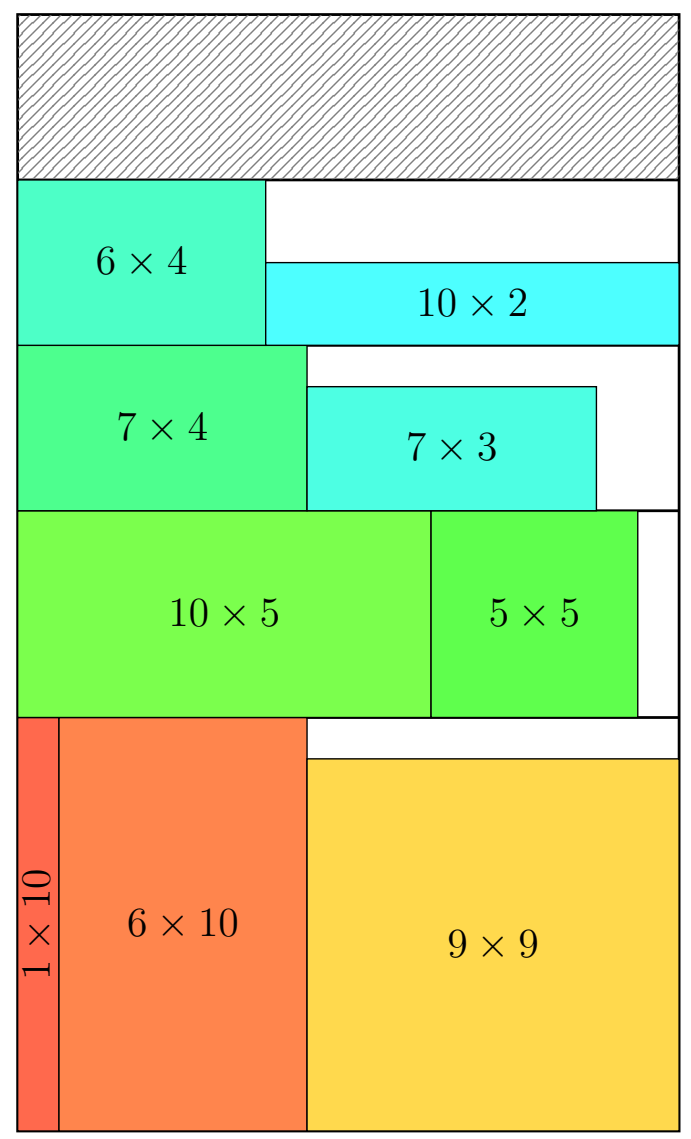

$16 \times 27$

Figura 3.17: Ilustração da solução da instância 15 do Modelo $\left(\mathcal{M}_{3}^{D E}\right)$ 


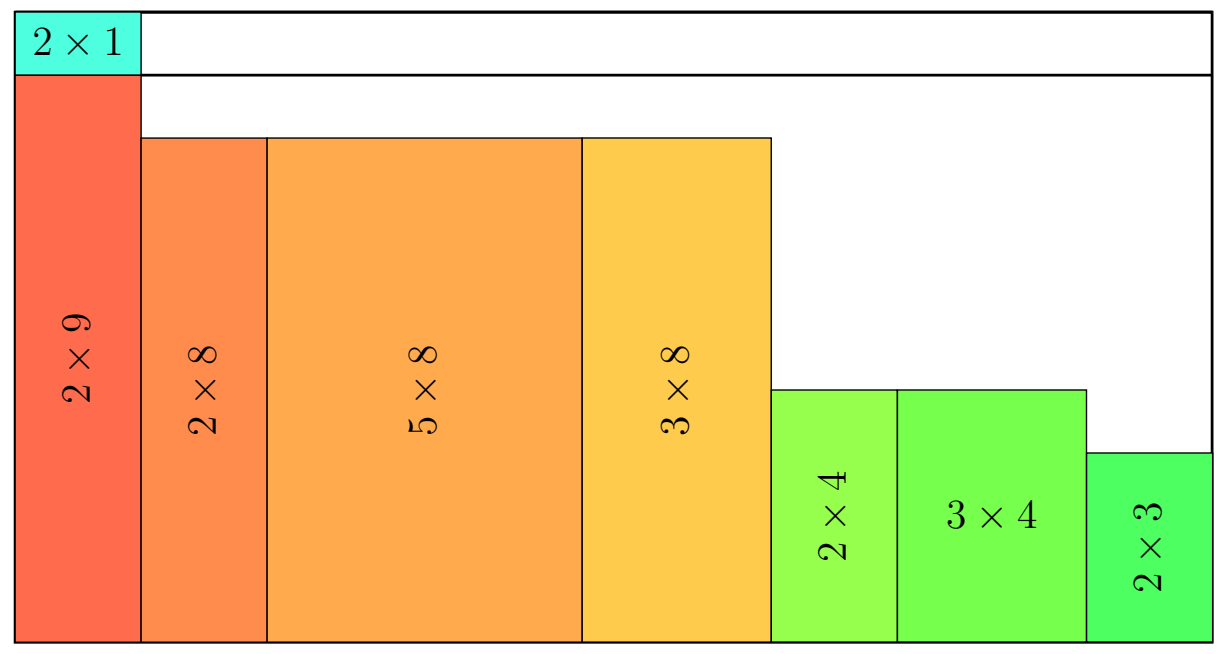

$19 \times 10$

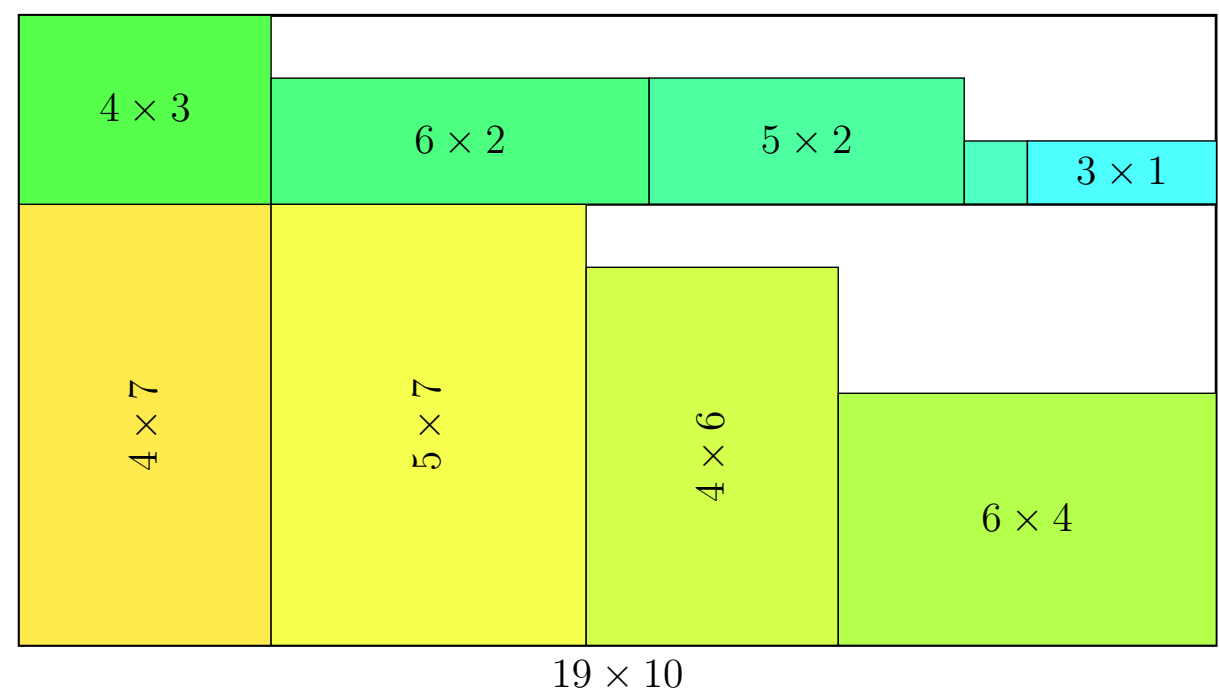

Figura 3.18: Ilustração da solução da instância 16 do Modelo $\left(\mathcal{M}_{3}^{D E}\right)$ 


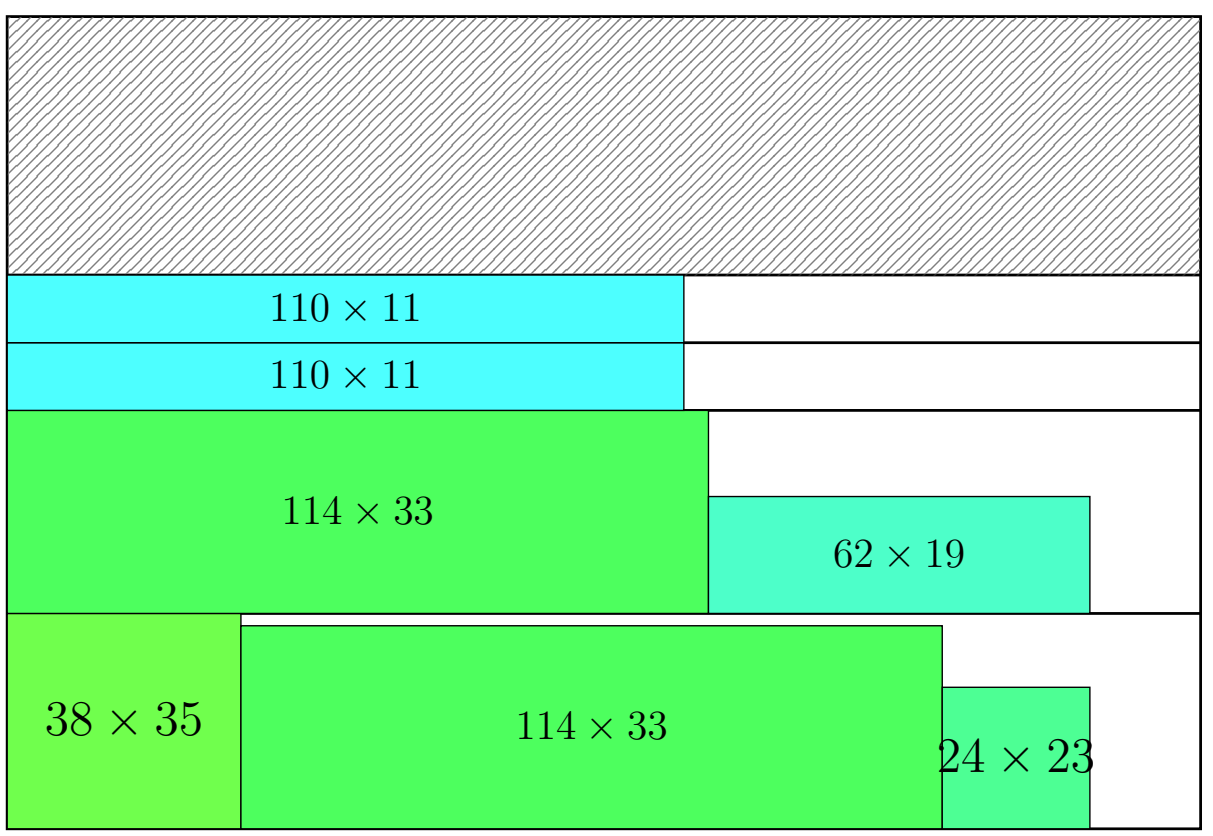

$194 \times 132$

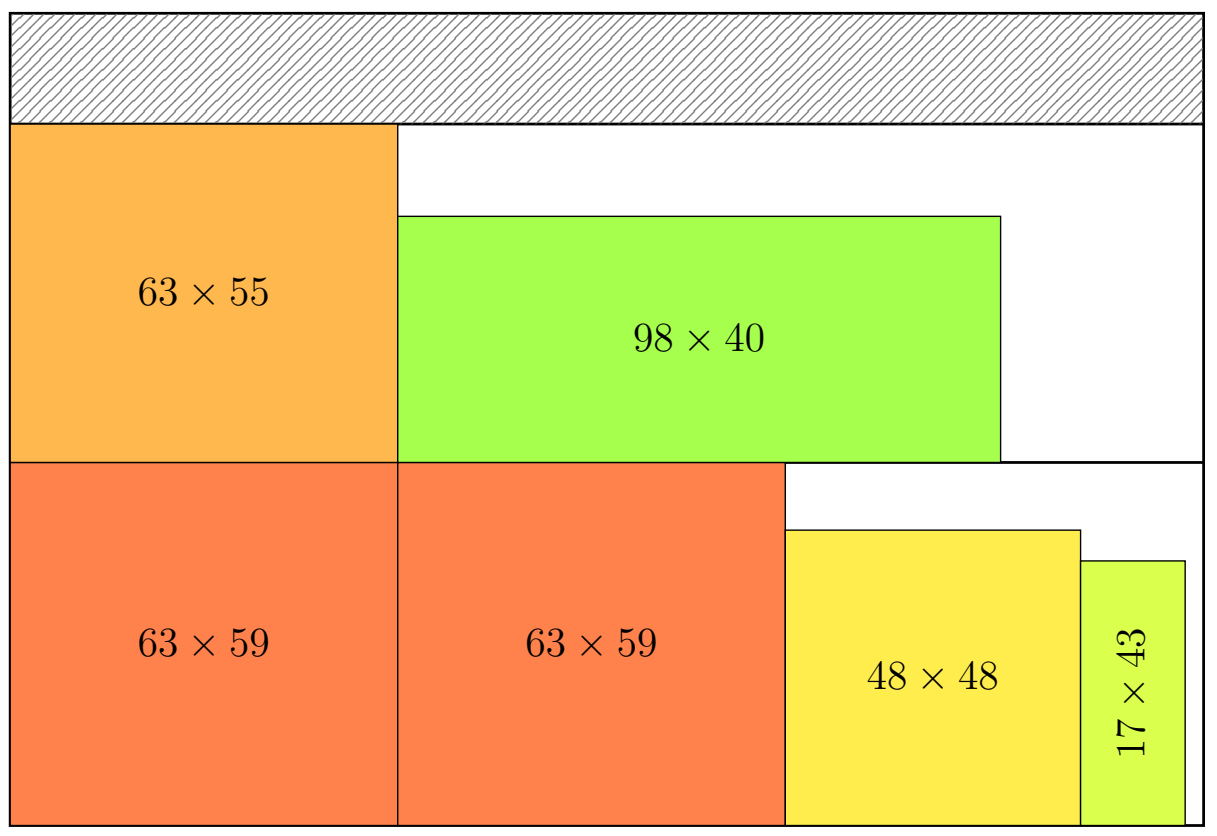

$194 \times 132$

Figura 3.19: Ilustração da solução da instância 17 do Modelo $\left(\mathcal{M}_{3}^{D E}\right)$ 


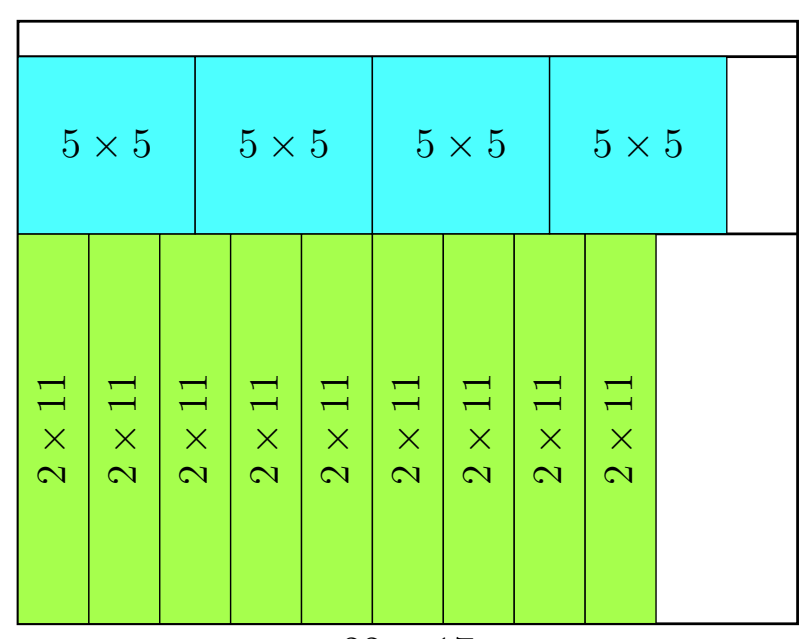

$22 \times 17$

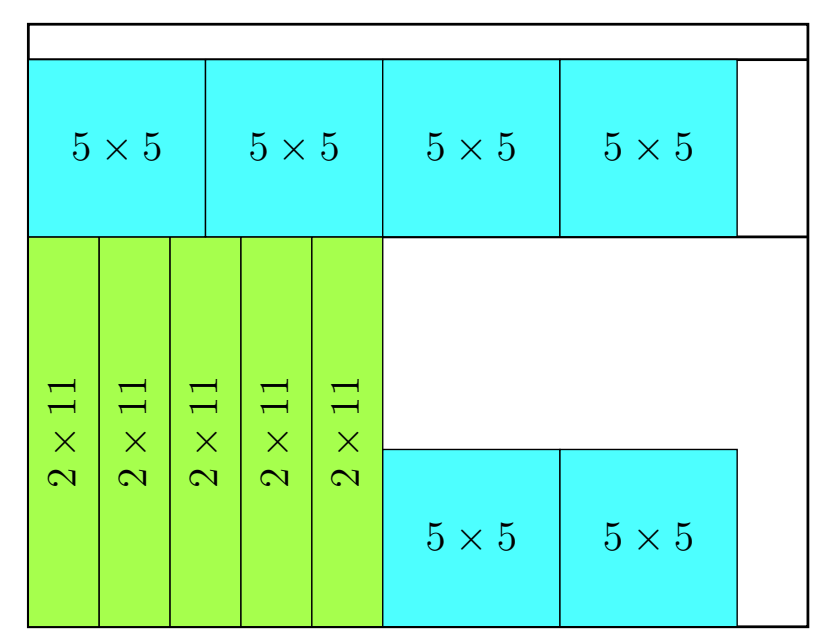

$22 \times 17$

Figura 3.20: Ilustração da solução da instância 18 do Modelo $\left(\mathcal{M}_{3}^{D E}\right)$ 


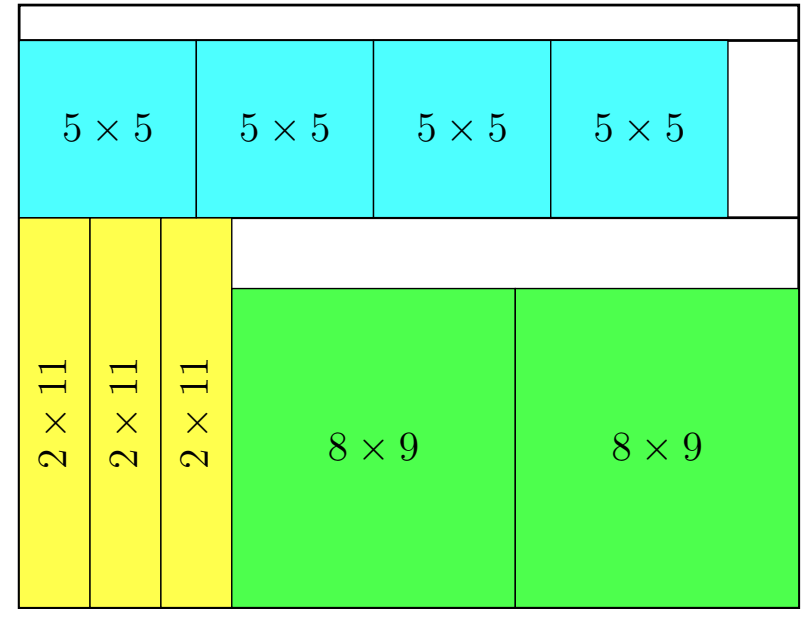

$22 \times 17$

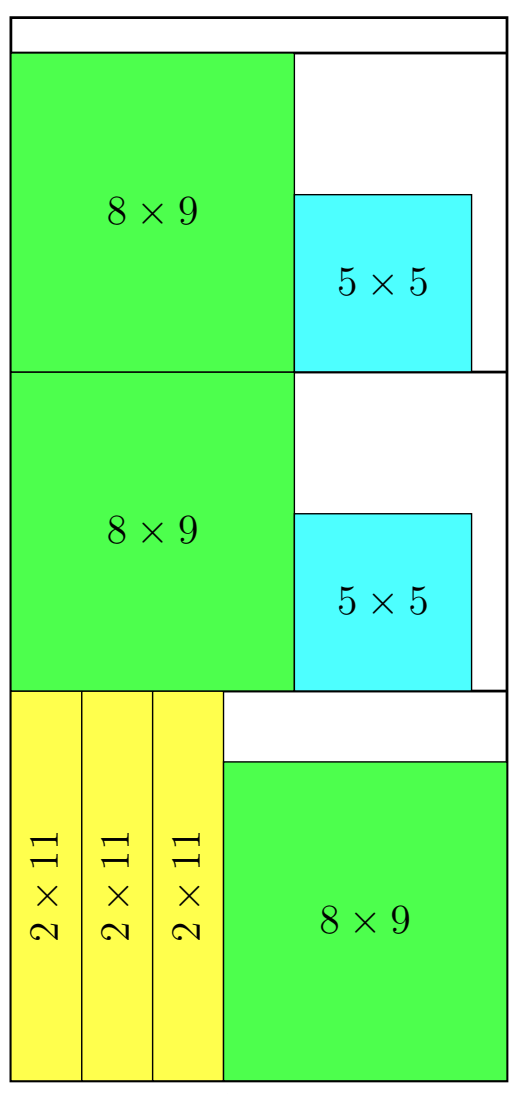

$14 \times 30$

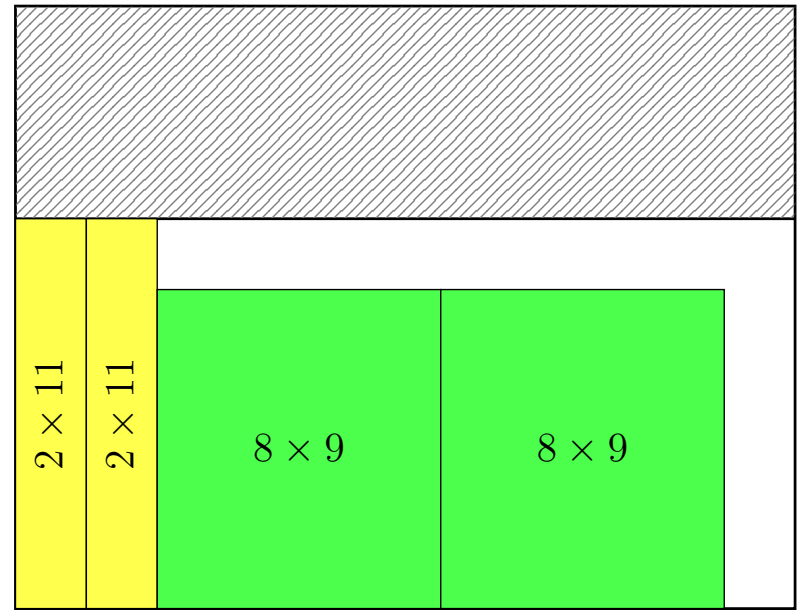

$22 \times 17$

Figura 3.21: Ilustração da solução da instância 20 do Modelo $\left(\mathcal{M}_{3}^{D E}\right)$ 


\section{Capítulo 4}

\section{Problemas de corte de itens retangulares com sobras aproveitáveis}

Neste capítulo desejamos estudar o problema de corte bidimensional não guilhotinado com sobras aproveitáveis. Este problema consiste em cortar um conjunto finito de itens retangulares a partir de um conjunto finito de objetos retangulares, com o objetivo de minimizar o custo dos objetos utilizados e, dentre as diferentes formas de se fazer isso, maximizar o valor das sobras aproveitáveis. Trata-se de uma versão generalizada do problema estudado no Capítulo 3.

Apresentamos inicialmente o problema de corte de itens retangulares a partir de objetos retangulares e um modelo de programação inteira mista para o mesmo. Em seguida consideramos as sobras aproveitáveis e apresentamos algumas propostas de modelos utilizando programação em dois níveis. Finalmente apresentamos reformulações dos modelos em dois níveis como problemas de programação inteira mista, bem como, experimentos numéricos.

\subsection{Um modelo para o problema de corte de itens retangulares}

Consideremos o problema de obter padrões de corte para produzir uma quantidade pré-determinada de itens retangulares a partir de objetos retangulares. O problema consiste em determinar a posição de cada item em algum objeto de forma que:

1. todo item seja atribuído a um objeto,

2. não haja sobreposição entre os itens atribuídos ao mesmo objeto,

3. o custo dos objetos utilizados seja mínimo.

Seja $p \geq 0$ a quantidade de objetos retangulares disponíveis; $H_{l}, W_{l}>0$ as dimensões horizontal e vertical de cada objeto $l$, para $l=1, \ldots, p ; c_{l}$ o custo do objeto $l ; n \geq 0$ a quantidade de itens retangulares que se deseja cortar e $h_{i}, w_{i}>0$ as dimensões horizontal e vertical de cada item $i$, para $i=1, \ldots, n$. Consideramos que as dimensões dos itens e dos objetos, bem como o custo dos objetos, são inteiros, uma vez que a precisão utilizada na prática é em geral de duas ou três casas decimais. Definimos $x_{i}, y_{i}$ como as variáveis que correspondem às coordenadas do centro de cada item retangular $i$. Definimos também $u_{l} \in\{0,1\}$ tal que:

$$
u_{l}=\left\{\begin{array}{l}
1, \text { se o objeto } l \text { é utilizado, } \\
0, \text { caso contrário }
\end{array}\right.
$$


e $\bar{u}_{i l} \in\{0,1\}$ tal que:

$$
\bar{u}_{i l}=\left\{\begin{array}{l}
1, \text { se o item } i \text { é produzido a partir do objeto } l \\
0, \text { caso contrário. }
\end{array}\right.
$$

Desejamos minimizar o custo dos objetos utilizados, dado por:

$$
\sum_{l=1}^{p} u_{l} c_{l} .
$$

Se os itens $i$ e $k$ forem produzidos a partir do mesmo objeto $l$, ou seja $\bar{u}_{i l}=\bar{u}_{k l}=1$, devemos impedir a sobreposição entre $i$ e $k$. Podemos modelar a não sobreposição da seguinte maneira:

$$
\begin{aligned}
y_{i}-y_{k} & \geq \frac{h_{i}+h_{k}}{2} \\
y_{k}-y_{i} & \geq \frac{h_{i}+h_{k}}{2} \quad \text { ou } \\
x_{i}-x_{k} & \geq \frac{w_{i}+w_{k}}{2} \\
x_{k}-x_{i} & \geq \frac{w_{i}+w_{k}}{2}, \quad k=1, \ldots, n, i=k+1, \ldots, n .
\end{aligned}
$$

Claramente (4.2) é equivalente a (2.2) e podemos reescrever a disjunção (4.2) através da técnica do $\mathrm{M}$ grande. A diferença neste caso é a implicação:

$$
\bar{u}_{i l}=\bar{u}_{k l}=1 \Rightarrow(4.2), l=1, \ldots, p, i=1, \ldots, n, k=i+1, \ldots, n .
$$

Consideramos as variáveis artificiais $\eta_{i k}, \bar{\eta}_{i k} \in\{0,1\}$ para modelar a disjunção das restrições de não sobreposição, através da técnica do M grande, como:

$$
\begin{aligned}
\frac{h_{i}+h_{k}}{2}-\left(y_{i}-y_{k}\right) \leq \eta_{i k} M_{l}^{H}+\bar{\eta}_{i k} M_{l}^{H}+\left(1-\bar{u}_{i l}\right) M_{l}^{H}+\left(1-\bar{u}_{k l}\right) M_{l}^{H}, \\
\frac{h_{i}+h_{k}}{2}+\left(y_{i}-y_{k}\right) \leq\left(1-\eta_{i k}\right) M_{l}^{H}+\bar{\eta}_{i k} M_{l}^{H}+\left(1-\bar{u}_{i l}\right) M_{l}^{H}+\left(1-\bar{u}_{k l}\right) M_{l}^{H}, \\
\frac{w_{i}+w_{k}}{2}-\left(x_{i}-x_{k}\right) \leq \eta_{i k} M_{l}^{W}+\left(1-\bar{\eta}_{i k}\right) M_{l}^{W}+\left(1-\bar{u}_{i l}\right) M_{l}^{W}+\left(1-\bar{u}_{k l}\right) M_{l}^{W}, \\
\frac{w_{i}+w_{k}}{2}+\left(x_{i}-x_{k}\right) \leq\left(1-\eta_{i k}\right) M_{l}^{W}+\left(1-\bar{\eta}_{i k}\right) M_{l}^{W}+\left(1-\bar{u}_{i l}\right) M_{l}^{W}+\left(1-\bar{u}_{k l}\right) M_{l}^{W},
\end{aligned}
$$

para todo $l=1, \ldots, p, i=1, \ldots, n, k=i+1, \ldots, n$, , no qual $M_{l}^{H}=H_{l}$ e $M_{l}^{W}$ são constantes suficientemente grandes.

Para garantir que um determinado item $i$ alocado ao objeto $l$ seja posicionado dentro deste objeto precisamos pedir que:

$$
\begin{aligned}
& y_{i}-\frac{h_{i}}{2} \geq 0, \\
& y_{i}+\frac{h_{i}}{2} \leq H_{l}+M_{l}^{h}\left(1-\bar{u}_{i l}\right), \\
& x_{i}-\frac{w_{i}}{2} \geq 0 \\
& x_{i}+\frac{w_{i}}{2} \leq W_{l}+M_{l}^{w}\left(1-\bar{u}_{i l}\right),
\end{aligned}
$$


para todo $i=1, \ldots, n, l=1, \ldots, p$. Observe que se $u_{i l}=0$ as restrições (4.8-4.11) não impõem nenhuma restrição no posicionamento dos retângulos. Por outro lado, se $u_{i l}=1$ elas impõem que o retângulo $i$ deve estar posicionado dentro dos limites horizontal e vertical do objeto $l$.

Desejamos também garantir que um item não seja atribuído a um objeto não utilizado. Isso pode ser modelado como:

$$
\bar{u}_{i l} \leq u_{l}, \text { para todo } i=1, \ldots, n \text { e } l=1, \ldots, p
$$

Também desejamos que a demanda de itens seja satisfeita e que cada item seja atribuído a apenas um objeto, ou seja:

$$
\sum_{l=1}^{p} \bar{u}_{i l}=1, \text { para todo } i=1, \ldots, n .
$$

Podemos escrever o problema em questão como:

$$
\text { Minimizar } \sum_{l=1}^{p} u_{l} c_{l}
$$

s.a

$$
\begin{array}{cl}
\frac{w_{i}}{2}-x_{i} \leq 0, & i=1, \ldots, n, \\
\frac{h_{i}}{2}-y_{i} \leq 0, & i=1, \ldots, n, \\
x_{i}+\frac{w_{i}}{2} \leq W_{k}+M_{l}^{W}\left(1-\bar{u}_{i l}\right), & i=1, \ldots, n, l=1, \ldots, p, \\
y_{i}+\frac{h_{i}}{2} \leq H_{k}+M_{l}^{H}\left(1-\bar{u}_{i l}\right), & i=1, \ldots, n, l=1, \ldots, p,
\end{array}
$$


O modelo (4.14-4.27) é uma forma de modelar o problema de corte de estoque bidimensional com múltiplos objetos. Nas próximas seções apresentaremos formas de modelar diferentes tipos de sobras aproveitáveis e como modelar o problema de corte com sobras.

\subsection{Considerando sobras aproveitáveis guilhotinadas}

O problema de corte apresentado na seção anterior consiste em obter padrões de corte para cortar $n$ retângulos (itens) a partir de $p$ placas (objetos), de forma a minimizar o custo relacionado às placas utilizadas. Esse problema pode possuir diversas soluções com custo mínimo. No problema de corte com sobras aproveitáveis desejamos obter aquela que maximiza o valor das sobras aproveitáveis. Nesta seção explicamos diferentes formas de modelar as sobras aproveitáveis e por fim apresentamos modelos de otimização para o problema.

Inicialmente consideramos que um padrão de corte para uma placa pode produzir nenhuma, uma ou duas sobras aproveitáveis. Consideramos também que apenas as regiões retangulares localizadas nas extremidades superior ou direita de um objeto, como ilustrado pelas regiões hachuradas na Figura 4.1, podem ser sobras aproveitáveis. Temos que $W_{l}$ e $H_{l}$ são as dimensões horizontais e verticais de um objeto $l$ e $\tilde{s}_{l}^{w}$ e $\tilde{s}_{l}^{h}$ são dimensões relacionadas às possíveis sobras aproveitáveis deste objeto, para $l=1, \ldots, p$. As possíveis sobras aproveitáveis de um objeto $l$ têm dimensões dadas por $W_{l} \times \tilde{s}_{l}^{h}$ e $\tilde{s}_{l}^{w} \times\left(H_{l}-\tilde{s}_{l}^{h}\right)$ (conforme ilustrado na Figura 4.1-(a)) ou dadas por $\left(W_{l}-\tilde{s}_{l}^{w}\right) \times \tilde{s}_{l}^{h}$ e $\tilde{s}_{l}^{w} \times H_{l}$ (conforme ilustrado na Figura 4.1-(b)).

Para caracterizar uma sobra como aproveitável, consideramos duas opções: (i) fornecer um par de dimensões mínimas $w_{\min } \times h_{\text {min }}$, de forma que para uma sobra ser considerada aproveitável, suas dimensões devem ser maiores ou iguais a essas dimensões mínimas ou (ii) fornecer uma lista de dimensões de itens $w_{1} \times h_{1}, w_{2} \times h_{2}, \ldots, w_{m} \times h_{m}$, para algum $m \geq 1$, de forma que para uma sobra ser considerada aproveitável, suas dimensões devem ser maiores ou iguais às dimensões de pelo menos um item da lista. Para modelar as sobras aproveitáveis, definimos para cada tipo de sobra uma função que deve valer zero se as dimensões de uma sobra não forem maiores ou iguais às dimensões mínimas consideradas (ou seja, a sobra não é aproveitável) e que deve valer a área da sobra em caso contrário (quando a sobra é aproveitável).

Primeiramente consideramos a possibilidade (i), tomando o par $w_{\min } \times h_{\min }$ como as dimensões horizontal e vertical mínimas que as regiões hachuradas precisam ter para serem consideradas sobras aproveitáveis.

Se $\tilde{s}_{l}^{h}<h_{\text {min }}$, a região hachurada com linhas na Figura 4.1 (a) não será uma sobra aproveitável. Similarmente, se $\tilde{s}_{l}^{w}<w_{\min }$ ou $H_{l}-\tilde{s}_{l}^{h}<h_{\min }$ a região hachurada com pontos a Figura 4.1 (a) não será uma sobra aproveitável. Considerando a Figura 4.1 (b), temos que se $\tilde{s}_{l}^{h}<h_{\text {min }}$ ou $W_{l}-\tilde{s}_{l}^{w}<w_{\min }$, a região hachurada com pontos não será sobra aproveitável e se $\tilde{s}_{l}^{w}<w_{\min }$, a região hachurada com linhas não será sobra aproveitável. Consideraremos separadamente cada um dos dois casos ilustrados na Figura 4.1, já que para cada objeto temos como possibilidades para as sobras ou a configuração ilustrada na Figura 4.1 (a) ou a configuração ilustrada na Figura 4.1 (b). Denotamos por $a^{L}\left(\tilde{s}_{l}^{h}, W_{l}\right)$ e $a^{P}\left(\tilde{s}_{l}^{w}, \tilde{s}_{l}^{h}, H_{l}\right)$ os valores das áreas das sobras aproveitáveis ilustradas com hachuras de linhas e pontos, respectivamente, na Figura 4.1 (a). Definimos $a^{L}(d, D)$ e $a^{P}\left(d_{1}, d_{2}, D\right)$ como:

$$
\begin{gathered}
a^{L}(d, D)= \begin{cases}d D, & \text { se } d \geq h_{\min } \\
0, & \text { caso contrário. }\end{cases} \\
a^{P}\left(d_{1}, d_{2}, D\right)= \begin{cases}d_{1}\left(D-d_{2}\right), & \text { se } d_{1} \geq w_{\min } \text { e } d_{2} \geq h_{\text {min }} \\
0, & \text { caso contrário. }\end{cases}
\end{gathered}
$$

Analogamente, denotamos por $b^{L}\left(\tilde{s}_{l}^{w}, H_{l}\right)$ e $b^{P}\left(\tilde{s}_{l}^{w}, \tilde{s}_{l}^{h}, W_{l}\right)$ os valores das áreas das sobras aprovei- 


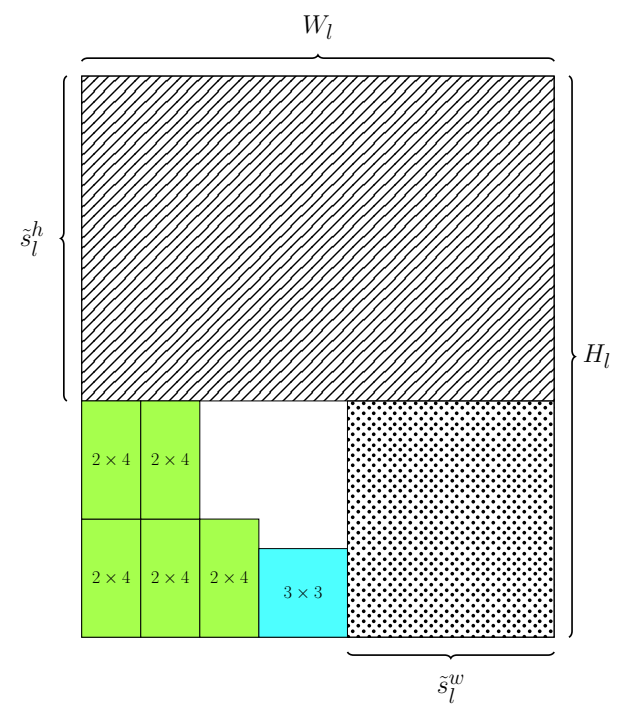

(a)

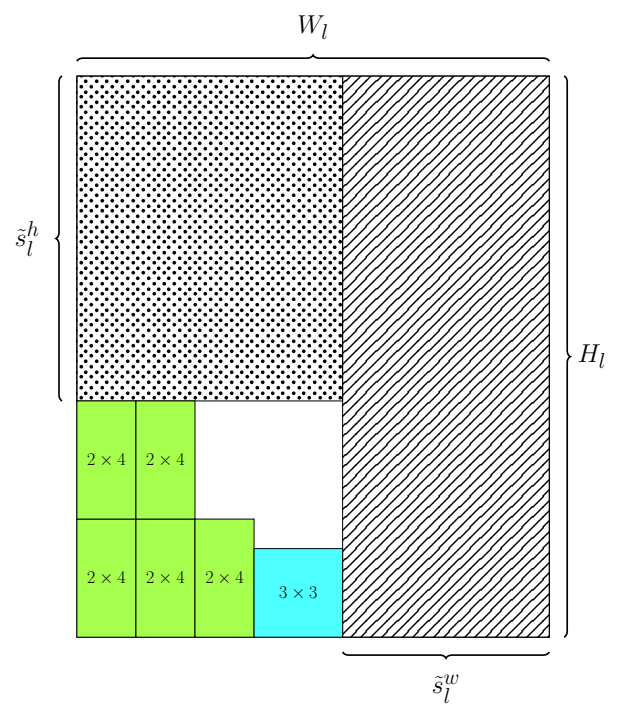

(b)

Figura 4.1: Duas possibilidades de sobras guilhotinadas para um objeto de dimensões $W_{l} \times H_{l}$. As sobras guilhotinadas são ilustradas pelas regiões hachuradas (com pontos e linhas). As caixas coloridas representam itens e a região em branco representa o descarte.

táveis ilustradas com hachuras de linhas e pontos, respectivamente, na Figura 4.1 (b) e definimos:

$$
\begin{gathered}
b^{L}(d, D)= \begin{cases}d D, & \text { se } d \geq w_{\text {min }} \\
0, & \text { caso contrário. }\end{cases} \\
b^{P}\left(d_{1}, d_{2}, D\right)= \begin{cases}d_{2}\left(D-d_{1}\right), & \text { se } d_{1} \geq w_{\text {min }} \text { e } d_{2} \geq h_{\text {min }} \\
0, & \text { caso contrário. }\end{cases}
\end{gathered}
$$

Para modelar o valor das sobras aproveitáveis, vamos considerar valores $\hat{c}_{l}$ associados a cada objeto $l$. Os valores $\hat{c}_{l}$ devem ser interpretados como pesos dados às sobras do objeto $l$. Modelamos o valor das sobras como sendo o valor da área da sobra multiplicada pelo valor $\hat{c}_{l}$. Considerando as áreas das sobras aproveitáveis modeladas de acordo com (4.28-4.31) podemos maximizar o valor das sobras aproveitáveis ao maximizar a seguinte função:

$$
\sum_{l=1}^{p} a^{L}\left(\tilde{s}_{l}^{h}, W_{l}\right) \hat{c}_{l}+a^{P}\left(\tilde{s}_{l}^{w}, \tilde{s}_{l}^{h}, H_{l}\right) \hat{c}_{l}+b^{L}\left(\tilde{s}_{l}^{w}, H_{l}\right) \hat{c}_{l}+b^{P}\left(\tilde{s}_{l}^{w}, \tilde{s}^{h}, W_{l}\right) \hat{c}_{l}
$$

Consideremos agora a possibilidade (ii), e sejam $w_{i} \times h_{i}$ as dimensões dos itens a serem empacotados, para $i=1, \ldots, n$. Denotamos por $\tilde{a}^{L}\left(\tilde{s}_{l}^{h}, W_{l}\right)$ e $\tilde{a}^{P}\left(\tilde{s}_{l}^{w}, \tilde{s}_{l}^{h}, H_{l}\right)$ os valores das áreas das sobras aproveitáveis ilustradas com hachuras de linhas e pontos, respectivamente, na Figura 4.1 (a) e definimos:

$$
\tilde{a}^{L}(d, D)= \begin{cases}d D, & \text { se } d \geq h_{1} \text { ou } \ldots \text { ou } d \geq h_{n} \\ 0, & \text { caso contrário }\end{cases}
$$

e

$$
\tilde{a}^{P}\left(d_{1}, d_{2}, D\right)= \begin{cases}d_{1} D-d_{1} d_{2}, & \text { se }\left(d_{1} \geq w_{1} \text { e } d_{2} \geq h_{1}\right) \text { ou } \ldots \text { ou }\left(d_{1} \geq w_{n} \text { e } d_{2} \geq h_{n}\right) \\ 0, & \text { caso contrário. }\end{cases}
$$

Analogamente, denotamos por $\tilde{b}^{L}\left(\tilde{s}_{l}^{w}, H_{l}\right)$ e $\tilde{b}^{P}\left(\tilde{s}_{l}^{w}, \tilde{s}_{l}^{h}, W_{l}\right)$ os valores das áreas das sobras aprovei- 
táveis ilustradas com hachuras de linhas e pontos, respectivamente, na Figura 4.1 (b) e definimos:

$$
\tilde{b}^{L}(d, D)= \begin{cases}d D, & \text { se } d \geq w_{1} \text { ou } \ldots \text { ou } d \geq w_{n} \\ 0, & \text { caso contrário }\end{cases}
$$

$\mathrm{e}$

$$
\tilde{b}^{P}\left(d_{1}, d_{2}, D\right)= \begin{cases}d_{2} D-d_{1} d_{2}, & \text { se }\left(d_{1} \geq w_{1} \text { e } d_{2} \geq h_{1}\right) \text { ou } \ldots \text { ou }\left(d_{1} \geq w_{n} \text { e } d_{2} \geq h_{n}\right), \\ 0, & \text { caso contrário. }\end{cases}
$$

Considerando como valor de uma sobra aproveitável de um objeto $l$ como sendo o valor $\hat{c}_{l}$ multiplicado pelo valor da área da sobra aproveitável, modelado de acordo com (4.33-4.36), podemos maximizar os valores das sobras aproveitáveis ao maximizar a seguinte função:

$$
\sum_{l=1}^{p} \tilde{a}^{L}\left(\tilde{s}_{l}^{h}, W_{l}\right) \hat{c}_{l}+\tilde{a}^{P}\left(\tilde{s}_{l}^{w}, \tilde{s}_{l}^{h}, H_{l}\right) \hat{c}_{l}+\tilde{b}^{l}\left(\tilde{s}_{l}^{w}, H_{l}\right) \hat{c}_{l}+\tilde{b}^{P}\left(\tilde{s}_{l}^{w}, \tilde{s}_{l}^{h}, W_{l}\right) \hat{c}_{l} .
$$

A seguir reescrevemos (4.28-4.31) e (4.33-4.36) como restrições de um modelo de otimização. Inicialmente apresentamos uma reformulação de (4.28-4.31) contendo termos não lineares. Em seguida explicamos como reescrever tais termos de forma linear. Por fim apresentamos a reformulação de (4.33-4.36), algumas propostas de modelos de otimização em dois níveis e suas reformulações em modelos de programação inteira mista para o problema de corte com sobras aproveitáveis guilhotinadas.

Considere as variáveis contínuas $A_{l}, B_{l}, \bar{A}_{l}, \bar{B}_{l}$ para $l=1, \ldots, p$. Temos que $A_{l}$ corresponde à $a^{L}\left(\tilde{s}_{l}^{h}, W_{l}\right), B_{l}$ corresponde à $a^{P}\left(\tilde{s}_{l}^{w}, \tilde{s}_{l}^{h}, H_{l}\right), \bar{A}$ corresponde à $b^{L}\left(\tilde{s}_{l}^{w}, H_{l}\right)$ e $\bar{B}_{l}$ corresponde à $b^{P}\left(\tilde{s}_{l}^{w}, \tilde{s}_{l}^{h}, W_{l}\right)$. Considere também as variáveis binárias $z_{l}, \bar{z}_{l} \in\{0,1\}$, bem como constantes $M_{l}^{A}, M_{l}^{W}$ e $M_{l}^{H}$ suficientemente grandes, para $l=1, \ldots, p$. Podemos reescrever (4.28-4.29), como restrições de um MIP, usando a técnica do M-grande, da seguinte forma:

$$
\begin{aligned}
\tilde{s}_{l}^{h} & \geq h_{\min }-M_{l}^{H} z_{l}, \\
A_{l} & \leq M_{l}^{A}\left(1-z_{l}\right), \\
H_{l}-\tilde{s}_{l}^{h} & \geq h_{\min }-M_{l}^{H} \bar{z}_{l}, \\
\tilde{s}_{l}^{w} & \geq w_{\min }-M_{l}^{W} \bar{z}_{l}, \\
B_{l} & \leq M_{l}^{A}\left(1-\bar{z}_{l}\right), \\
\tilde{s}_{l}^{h} & \geq 0 \\
\tilde{s}_{l}^{w} & \geq 0 \\
A_{l} & \leq W_{l} \tilde{s}_{l}^{h} \\
B_{l} & \leq H_{l} \tilde{s}_{l}^{w}-\tilde{s}_{l}^{h} \tilde{s}_{l}^{w}, \\
A_{l} & \geq 0 \\
B_{l} & \geq 0
\end{aligned}
$$

para $l=1, \ldots, p$. Dependendo do valor atribuído à variável $z_{l}$ é imposta uma das condições de (4.28). Se $z_{l}=0$ as restrições (4.38) e (4.45), juntamente com a maximização do valor das sobras aproveitáveis impõem que $a^{L}\left(\tilde{s}^{h}, W_{l}\right)=W_{l} \tilde{s}^{h}$. Se $z_{l}=1$ as restrições (4.39) e (4.47) impõem $a^{L}\left(\tilde{s}^{h}, W_{l}\right)=0$. Similarmente, a atribuição de valor à variável $\bar{z}_{l}$ impõe se $a^{P}\left(\tilde{s}^{w}, \tilde{s}^{h}, H_{l}\right)$ deve valer zero ou se deve valer $H_{l} \tilde{s}_{l}^{w}-\tilde{s}_{l}^{h} \tilde{s}_{l}^{w}$.

Podemos também escrever (4.30-4.31) como restrições de um problema de otimização da 
seguinte forma:

$$
\begin{aligned}
\tilde{s}_{l}^{w} & \geq w_{\min }-M_{l}^{H} \bar{z}_{l}, \\
\bar{A}_{l} & \leq M_{l}^{A}\left(1-\bar{z}_{l}\right), \\
W_{l}-\tilde{s}_{l}^{w} & \geq w_{\min }-M_{l}^{W} z_{l}, \\
\tilde{s}_{l}^{h} & \geq h_{\min }-M_{l}^{H} z_{l}, \\
\bar{B}_{l} & \leq M_{l}^{A}\left(1-z_{l}\right) . \\
\tilde{s}_{l}^{h} & \geq 0 \\
\tilde{s}_{l}^{w} & \geq 0 \\
\bar{A}_{l} & \leq H_{l} \tilde{s}_{l}^{w}, \\
\bar{B}_{l} & \leq W_{l} \tilde{s}_{l}^{h}-\tilde{s}_{l}^{h} \tilde{s}_{l}^{w}, \\
\bar{A}_{l} & \geq 0, \\
\bar{B}_{l} & \geq 0,
\end{aligned}
$$

para $l=1, \ldots, p$. Da mesma forma que em (4.38-4.48), em (4.49-4.57) a atribuição de valores às variáveis $z_{l}$ e $\bar{z}_{l}$ controla se $b^{L}\left(\tilde{s}^{w}, H_{l}\right)$ e $b^{P}\left(\tilde{s}^{w}, \tilde{s}^{h}, W_{l}\right)$, respectivamente, valem zero ou correspondem à área da sobra aproveitável correspondente.

Como dissemos anteriormente, desejamos que as sobras sejam conforme o ilustrado na Figura 4.1 (a) ou conforme o ilustrado na Figura 4.1 (b). Mais precisamente queremos impor (4.38-4.42) ou (4.49-4.53). Note que os limitantes inferiores para $\tilde{s}_{l}^{w}$ e $\tilde{s}_{l}^{h}$ e os limitantes inferiores e superiores para $A_{l}, B_{l}, \bar{A}_{l}$ e $\bar{B}_{l}$, ou seja, as restrições (4.43-4.48) e (4.54-4.57) devem ser impostas sempre. Para modelar essa disjunção, consideramos $\tilde{z}_{l} \in\{0,1\}$ e reescrevemos (4.38-4.42) e (4.49-4.53) da seguinte forma:

$$
\begin{aligned}
\tilde{s}_{l}^{h} & \geq h_{\min }-M_{l}^{H} z_{l}-M_{l}^{H} \tilde{z}_{l}, \\
A_{l} & \leq M_{l}^{H}\left(1-z_{l}\right), \\
A_{l} & \leq M_{l}^{A}\left(1-\tilde{z}_{l}\right) \\
H_{l}-\tilde{s}_{l}^{h} & \geq h_{\min }-M_{l}^{H} \bar{z}_{l}-M_{l}^{H} \tilde{z}_{l}, \\
\tilde{s}_{l}^{w} & \geq w_{\min }-M_{l}^{W} \bar{z}_{l}-M_{l}^{W} \tilde{z}_{l}, \\
B_{l} & \leq M_{l}^{H}\left(1-\bar{z}_{l}\right), \\
B_{l} & \leq M_{l}^{A}\left(1-\tilde{z}_{l}\right) \\
\tilde{s}_{l}^{w} & \geq w_{\min }-M_{l}^{W} \bar{z}_{l}-M_{l}^{W}\left(1-\tilde{z}_{l}\right), \\
\bar{A}_{l} & \leq M_{l}^{H}\left(1-\bar{z}_{l}\right), \\
\bar{A}_{l} & \leq M_{l}^{A} \tilde{z}_{l}, \\
W_{l}-\tilde{s}_{l}^{w} & \geq h_{\min }-M_{l}^{W} z_{l}-M_{l}^{W}\left(1-\tilde{z}_{l}\right), \\
\tilde{s}_{l}^{h} & \geq h_{\min }-M_{l}^{H} z_{l}-M_{l}^{H}\left(1-\tilde{z}_{l}\right), \\
\bar{B}_{l} & \leq M_{l}^{H}\left(1-z_{l}\right), \\
\bar{B}_{l} & \leq M_{l}^{A} \tilde{z}_{l}, \\
\tilde{s}_{l}^{w}, \tilde{s}_{l}^{h}, A_{l}, B_{l}, \bar{A}_{l}, \bar{B}_{l} & \geq 0,
\end{aligned}
$$

para $l=1, \ldots, p$. Note que se $\tilde{z}_{l}=0$ então (4.58-4.71) implica em (4.38-4.42) e, caso $\tilde{z}_{l}=1$, implica em (4.49-4.53).

As inequações (4.46) e (4.55) possuem ambas o termo não linear $\tilde{s}_{l}^{h} \tilde{s}_{l}^{w}$. Uma vez que os ob- 
jetos e itens considerados possuem apenas dimensões inteiras e que as dimensões das sobras são maximizadas, podemos supor, sem perda de generalidade, que há uma solução ótima em que $\tilde{s}_{l}^{w} \mathrm{e}$ $\tilde{s}_{l}^{h}$ têm valores inteiros. Portanto, é possível linearizar o termo não linear $\tilde{s}_{l}^{h} \tilde{s}_{l}^{w}$ através da expansão binária de um de seus termos e uma manipulação algébrica [Harjunkoski97, Yanasse06]. Vamos escrever expansão binária de $\tilde{s}_{l}^{w}$ :

$$
\tilde{s}_{l}^{w}=\sum_{i=0}^{n b\left(\tilde{s}_{l}^{w}\right)} 2^{i} \tilde{\beta}_{i l}, \quad \tilde{\beta}_{i l} \in\{0,1\},
$$

tal que $n b(k)=\left(\left\lfloor\log _{2}(k)\right\rfloor+1\right)$ é o número mínimo de bits necessários para a representação binária de $k$. Substituindo $\tilde{s}_{l}^{w}$ por (4.72) no termo não linear $\tilde{s}_{l}^{h} \tilde{s}_{l}^{w}$. temos:

$$
\tilde{s}_{l}^{h} \tilde{s}_{l}^{w}=\left(\sum_{i=0}^{n b\left(\tilde{s}_{l}^{w}\right)} 2^{i} \tilde{\beta}_{i l}\right) \tilde{s}_{l}^{h}
$$

Considerando $\tilde{\alpha}_{i l}$ definido como:

$$
\tilde{\alpha}_{i l}= \begin{cases}\tilde{s}_{l}^{h}, & \text { se } \tilde{\beta}_{i l}=1, \\ 0, & \text { caso contrário, }\end{cases}
$$

podemos reescrever $\tilde{s}_{l}^{h} \tilde{s}_{l}^{w}$ como $\sum_{i=0}^{n b\left(\tilde{s}_{l}^{w}\right)} 2^{i} \tilde{\alpha}_{i l}$. Dessa forma, (4.46) e (4.55) podem ser substituídas por :

$$
\begin{aligned}
B_{l} & \leq H_{l} \tilde{s}_{l}^{w}-\sum_{i=0}^{n b\left(\tilde{s}_{l}^{w}\right)} 2^{i} \tilde{\alpha}_{i l}, \\
\bar{B}_{l} & \leq W_{l} \tilde{s}_{l}^{h}-\sum_{i=0}^{n b\left(\tilde{s}_{l}^{w}\right)} 2^{i} \tilde{\alpha}_{i l}, \\
\tilde{\alpha}_{i l} & \leq \tilde{s}_{l}^{h}, \\
\tilde{\alpha}_{i l} & \geq \tilde{s}_{l}^{h}-\left(1-\tilde{\beta}_{i l}\right) M_{l}^{H}, \\
\tilde{\alpha}_{i l} & \leq \tilde{\beta}_{i l} M_{l}^{H}, \\
\tilde{\alpha}_{i l} & \geq 0,
\end{aligned}
$$

para $l=1, \ldots, p$.

Apresentamos, a seguir, a reformulação MIP de (4.33-4.36), que caracterizam sobras aproveitáveis como itens com dimensões mínimas horizontais e verticais $w_{\min }$ e $h_{\min }$, respectivamente. Consideramos as variáveis $A_{l}$ para modelar o valor de $\tilde{a}^{L}\left(\tilde{s}_{l}^{h}, W_{l}\right), B_{l}$ para modelar o valor de $\tilde{a}^{P}\left(\tilde{s}_{l}^{w}, \tilde{s}_{l}^{h}, H_{l}\right), \bar{A}_{l}$ para modelar o valor de $\tilde{b}^{L}\left(\tilde{s}_{l}^{w}, H_{l}\right)$ e $\bar{B}_{l}$ para modelar o valor de $\tilde{b}^{P}\left(\tilde{s}_{l}^{w}, \tilde{s}_{l}^{h}, W_{l}\right)$. Dessa forma, podemos reescrever (4.33-4.34), como restrições de um MIP, de forma similar à 
realizada com (4.28-4.29):

$$
\begin{aligned}
\tilde{s}_{l}^{h} & \geq h_{i}-M_{l}^{H} \tau_{i l}, \\
A_{l} & \leq M_{l}^{A}\left(1-\tau_{1 l}\right)+\ldots+M_{l}^{A}\left(1-\tau_{n l}\right), \\
H_{l}-\tilde{s}_{l}^{h} & \geq h_{i}-M_{l}^{H} \bar{\tau}_{i l}, \\
\tilde{s}_{l}^{w} & \geq w_{i}-M_{l}^{W} \bar{\tau}_{i l}, \\
B_{l} & \leq M_{l}^{A}\left(1-\bar{\tau}_{1 l}\right)+\ldots+M_{l}^{A}\left(1-\bar{\tau}_{n l}\right), \\
\tilde{s}_{l}^{h} & \geq 0, \\
\tilde{s}_{l}^{w} & \geq 0 \\
A_{l} & \leq W_{l} \tilde{s}_{l}^{h}, \\
B_{l} & \leq H_{l} \tilde{s}_{l}^{w}-\tilde{s}_{l}^{h} \tilde{s}_{l}^{w}, \\
A_{l} & \geq 0, \\
B_{l} & \geq 0, \\
\tau_{1 l}, \bar{\tau}_{1 l} & \in\{0,1\}, i=0, \ldots, n,
\end{aligned}
$$

para $l=1, \ldots, p$. Temos que se $\tau_{i l}=0$, a sobra do objeto $l$ dada por $W_{l} \times \tilde{s}_{l}^{h}$, pode acomodar o item $i$. Para $l$ fixo e $i=1, \ldots, n$, se $\tau_{i l}=1$, temos que $A_{l}=0$. Equivalentemente, se $\bar{\tau}_{i l}=0$, a sobra do objeto $l$ dada por $\tilde{s}_{l}^{w} \times\left(H_{l}-\tilde{s}_{l}^{h}\right)$, pode acomodar o item $i$. Para $l$ fixo e $i=1, \ldots, n$, se $\overline{\tau_{i l}}=1$, temos que $B_{l}=0$.

Podemos também escrever (4.35-4.36) como restrições de um problema de otimização da seguinte forma:

$$
\begin{aligned}
\tilde{s}_{l}^{w} & \geq w_{i}-M_{l}^{W} \bar{\tau}_{i l}, \\
\bar{A}_{l} & \leq M_{l}^{A}\left(1-\bar{\tau}_{1 l}\right)+\ldots+M_{l}^{A}\left(1-\bar{\tau}_{n l}\right), \\
W_{l}-\tilde{s}_{l}^{w} & \geq w_{i}-M_{l}^{W} \tau_{i l}, \\
\tilde{s}_{l}^{h} & \geq h_{i}-M_{l}^{H} \tau_{i l}, \\
\bar{B}_{l} & \leq M_{l}^{A}\left(1-\tau_{1 l}\right)+\ldots+M_{l}^{A}\left(1-\tau_{n l}\right) . \\
\bar{A}_{l} & \leq H_{l} \tilde{s}_{l}^{w}, \\
\bar{B}_{l} & \leq W_{l} \tilde{s}_{l}^{h}-\tilde{s}_{l}^{h} \tilde{s}_{l}^{w}, \\
\bar{A}_{l} & \geq 0, \\
\bar{B}_{l} & \geq 0, \\
\tilde{s}_{l}^{h} & \geq 0, \\
\tilde{s}_{l}^{w} & \geq 0, \\
\tau_{1 l}, \bar{\tau}_{1 l} & \in\{0,1\}, i=0, \ldots, n,
\end{aligned}
$$

para $l=1, \ldots, p$. Temos que se $\tau_{i l}=0$, a sobra do objeto $l$ dada por $H_{l} \times \tilde{s}_{l}^{w}$, pode acomodar o item $i$. Para $l$ fixo e $i=1, \ldots, n$, se $\tau_{i l}=1$, temos que $\bar{A}_{l}=0$. Equivalentemente, se $\bar{\tau}_{i l}=0$, a sobra do objeto $l$ dada por $\tilde{s}_{l}^{h} \times\left(W_{l}-\tilde{s}_{l}^{w}\right)$, pode acomodar o item $i$. Para $l$ fixo e $i=1, \ldots, n$, se $\bar{\tau}_{i l}=1$, temos que $\bar{B}_{l}=0$.

Da mesma forma que fizemos ao considerar (4.28-4.31), desejamos que as sobras sejam conforme ilustrado na Figura 4.1 (a) ou conforme ilustramos na Figura 4.1 (b). Para isso considerare- 
mos novamente as variáveis $\tilde{z}_{l}$, e reescreveremos (4.79-4.83) e (4.91-4.95). da seguinte forma:

$$
\begin{aligned}
\tilde{s}_{l}^{h} & \geq h_{i}-M_{l}^{H} \tau_{i l}-M_{l}^{H} \tilde{z}_{l}, \\
A_{l} & \leq M_{l}^{A}\left(1-\tau_{1 l}\right)+\ldots+M_{l}^{A}\left(1-\tau_{n l}\right) \\
A_{l} & \leq M_{l}^{A}\left(1-\tilde{z}_{l}\right), \\
H_{l}-\tilde{s}_{l}^{h} & \geq h_{i}-M_{l}^{H} \bar{\tau}_{i l}-M_{l}^{H} \tilde{z}_{l}, \\
\tilde{s}_{l}^{w} & \geq w_{i}-M_{l}^{W} \bar{\tau}_{i l}-M_{l}^{W} \tilde{z}_{l}, \\
B_{l} & \leq M_{l}^{A}\left(1-\bar{\tau}_{1 l}\right)+\ldots+M_{l}^{A}\left(1-\bar{\tau}_{n l}\right), \\
B_{l} & \leq M_{l}^{A}\left(1-\tilde{z}_{l}\right), \\
\tilde{s}_{l}^{w} & \geq w_{i}-M_{l}^{W} \bar{\tau}_{i l}-M_{l}^{W}\left(1-\tilde{z}_{l}\right), \\
\bar{A}_{l} & \leq M_{l}^{A}\left(1-\bar{\tau}_{1 l}\right)+\ldots+M_{l}^{A}\left(1-\bar{\tau}_{n l}\right), \\
\bar{A}_{l} & \leq M_{l}^{A} \tilde{z}_{l} \\
W_{l}-\tilde{s}_{l}^{w} & \geq h_{i}-M_{l}^{W} \tau_{i l}-M_{l}^{W}\left(1-\tilde{z}_{l}\right), \\
\tilde{s}_{l}^{h} & \geq h_{i}-M_{l}^{H} \tau_{i l}-M_{l}^{H}\left(1-\tilde{z}_{l}\right), \\
\bar{B}_{l} & \leq M_{l}^{A}\left(1-\tau_{1 l}\right)+\ldots+M_{l}^{A}\left(1-\tau_{1 l}\right), \\
\bar{B}_{l} & \leq M_{l}^{A} \tilde{z}_{l}, \\
\bar{A}_{l}, \bar{B}_{l} & \geq 0,
\end{aligned}
$$

para $l=1, \ldots, p$. Note que se $\tilde{z}_{l}=0$ estamos impondo as condições (4.79-4.85) e caso $\tilde{z}_{l}=1$ impomos (4.91-4.95). Temos que (4.33-4.34) podem ser modeladas como restrições de um MIP através das inequações (4.100-4.113), (4.84-4.89) e (4.96-4.99). Note que as inequações (4.87) e (4.97) podem ser linearizadas reescrevendo-as como (4.73-4.78).

É natural escrever o problema de corte com sobras aproveitáveis como um problema de otimização em dois níveis. uma vez que desejamos obter um padrão de corte que maximize o reaproveitamento de material, dentre aqueles que minimizam o custo relacionado às placas utilizadas. No nível superior desejamos maximizar as sobras aproveitáveis e no nível inferior desejamos obter um padrão de corte que minimize o custo dos objetos utilizados. Apresentamos a seguir dois modelos de programação em dois níveis (um para cada forma de definir as sobras aproveitáveis, conforme explicado em (i) e (ii) anteriormente) e em seguida suas reformulações em modelos de programação inteira mista. Nos dois modelos de programação em dois níveis que apresentamos, a função objetivo do problema do nível superior consiste em maximizar

$$
\sum_{l=1}^{p} A_{l} \hat{c}_{l}+B_{l} \hat{c}_{l}+\bar{A}_{l} \hat{c}_{l}+\bar{B}_{l} \hat{c}_{l} .
$$

Se considerarmos os valores das sobras, conforme o caso (i), temos que o problema do nível superior deve ter como restrições as inequações (4.58-4.71), (4.43-4.45), (4.47-4.48), (4.54-4.57) e (4.73-4.78). Se considerarmos os valores das sobras, conforme o caso (ii), temos que o problema do nível superior deve ter como restrições (4.100-4.113), (4.84-4.86), (4.88-4.89), (4.96-4.99) e (4.73-4.78). O problema do nível inferior, em ambos os casos, corresponde a uma versão modificada de (4.14-4.27) considerando as dimensões das sobras.

Portanto, o modelo de programação em dois níveis para o problema de corte com sobras aproveitáveis guilhotinadas, considerando as sobras modeladas de acordo com (i), pode ser escrito como: 


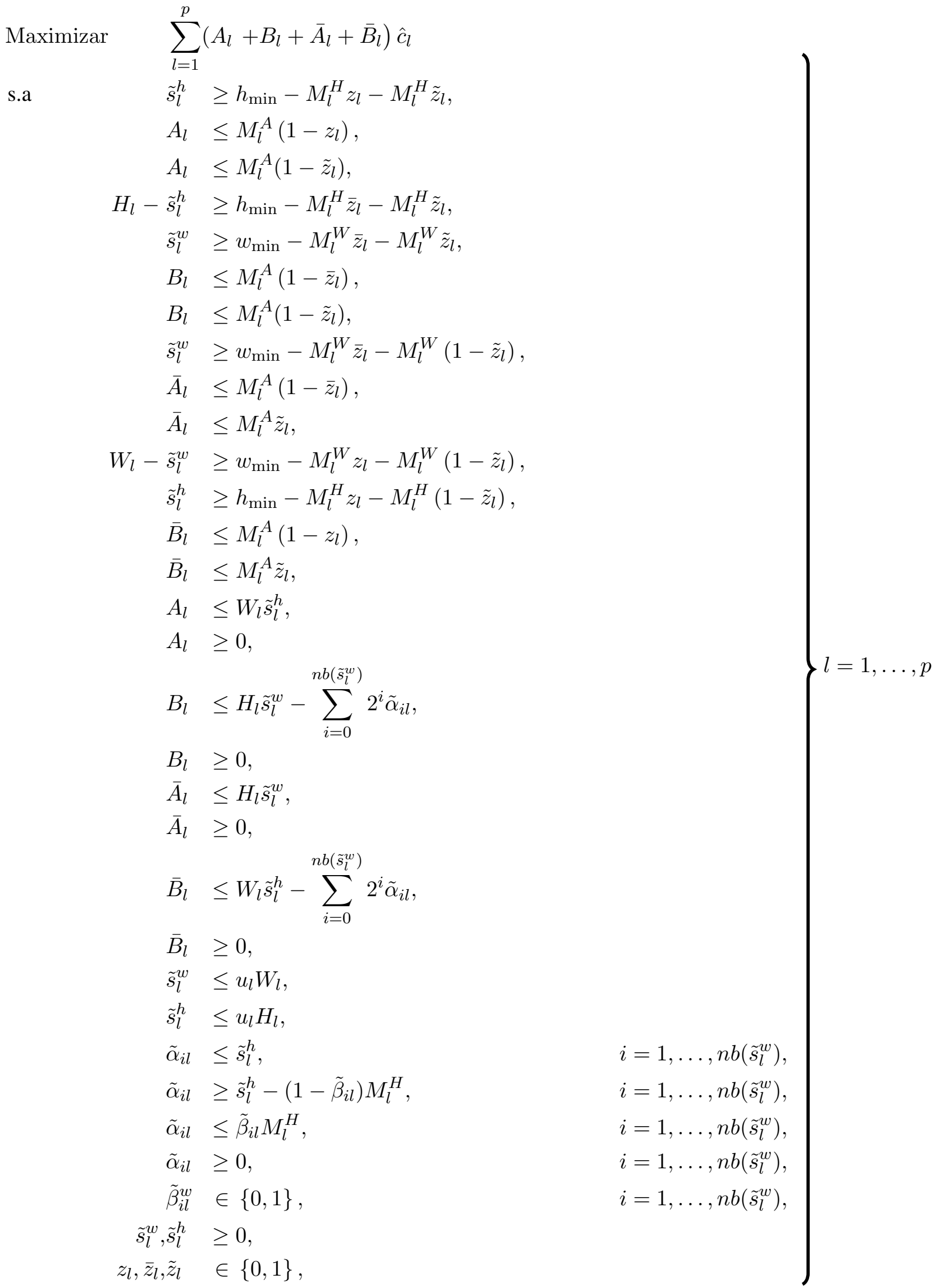




$$
\begin{aligned}
& \left(x, y, u, \bar{s}^{w}, \bar{s}^{h}\right) \in \operatorname{argmin} \sum_{l=1}^{p} u_{l} c_{l} \\
& \text { s.a } \quad \frac{h_{i}+h_{k}}{2}-\left(y_{i}-y_{k}\right) \leq \eta_{i k} M_{l}^{H}+\bar{\eta}_{i k} M_{l}^{H} \\
& +\left(1-\bar{u}_{i l}\right) M_{l}^{H}+\left(1-\bar{u}_{k l}\right) M_{l}^{H}, \\
& i=1, \ldots, n, k=i+1, \ldots, n, l=1, \ldots, p \\
& \frac{h_{i}+h_{k}}{2}+\left(y_{i}-y_{k}\right) \leq\left(1-\eta_{i k}\right) M_{l}^{H}+\bar{\eta}_{i k} M_{l}^{H} \\
& +\left(1-\bar{u}_{i l}\right) M_{l}^{H}+\left(1-\bar{u}_{k l}\right) M_{l}^{H}, \\
& i=1, \ldots, n, k=i+1, \ldots, n, l=1, \ldots, p \\
& \frac{w_{i}+w_{k}}{2}-\left(x_{i}-x_{k}\right) \leq \eta_{i k} M_{l}^{W}+\left(1-\bar{\eta}_{i k}\right) M_{l}^{W} \\
& +\left(1-\bar{u}_{i l}\right) M_{l}^{W}+\left(1-\bar{u}_{k l}\right) M_{l}^{W}, \\
& i=1, \ldots, n, k=i+1, \ldots, n, l=1, \ldots, p \\
& \frac{w_{i}+w_{k}}{2}+\left(x_{i}-x_{k}\right) \leq\left(1-\eta_{i k}\right) M_{l}^{W}+\left(1-\bar{\eta}_{i k}\right) M_{l}^{W} \\
& +\left(1-\bar{u}_{i l}\right) M_{l}^{W}+\left(1-\bar{u}_{k l}\right) M_{l}^{W}, \\
& i=1, \ldots, n, k=i+1, \ldots, n, l=1, \ldots, p \\
& y_{i}-\frac{h_{i}}{2} \geq 0, i=1, \ldots, n, \\
& y_{i}+\frac{h_{i}}{2} \leq H_{l}-\tilde{s}_{l}^{h}+M_{l}^{h}\left(1-\bar{u}_{i l}\right), \\
& i=1, \ldots, n, l=1, \ldots, p \\
& x_{i}-\frac{w_{i}}{2} \geq 0, i=1, \ldots, n, \\
& x_{i}+\frac{w_{i}}{2} \leq W_{l}-\tilde{s}_{l}^{w}+M_{l}^{w}\left(1-\bar{u}_{i l}\right), \\
& i=1, \ldots, n, l=1, \ldots, p \\
& \bar{u}_{i l} \leq u_{l}, i=1, \ldots, n, l=1, \ldots, p, \\
& \sum_{l=1}^{p} \bar{u}_{i l} \leq 1, i=1, \ldots, n . \\
& u_{j} \in\{0,1\}, j=1, \ldots, p, \\
& \bar{u}_{i j} \in\{0,1\}, i=1, \ldots, n, j=1, \ldots, p, \\
& \eta_{i k}, \bar{\eta}_{i k} \in\{0,1\}, i=1, \ldots, n, k=i+1, \ldots, n .
\end{aligned}
$$

Similarmente, o modelo em dois níveis para o problema de corte com sobras aproveitáveis guilhotinadas, considerando as sobras modeladas de acordo com (ii), pode ser escrito como: 


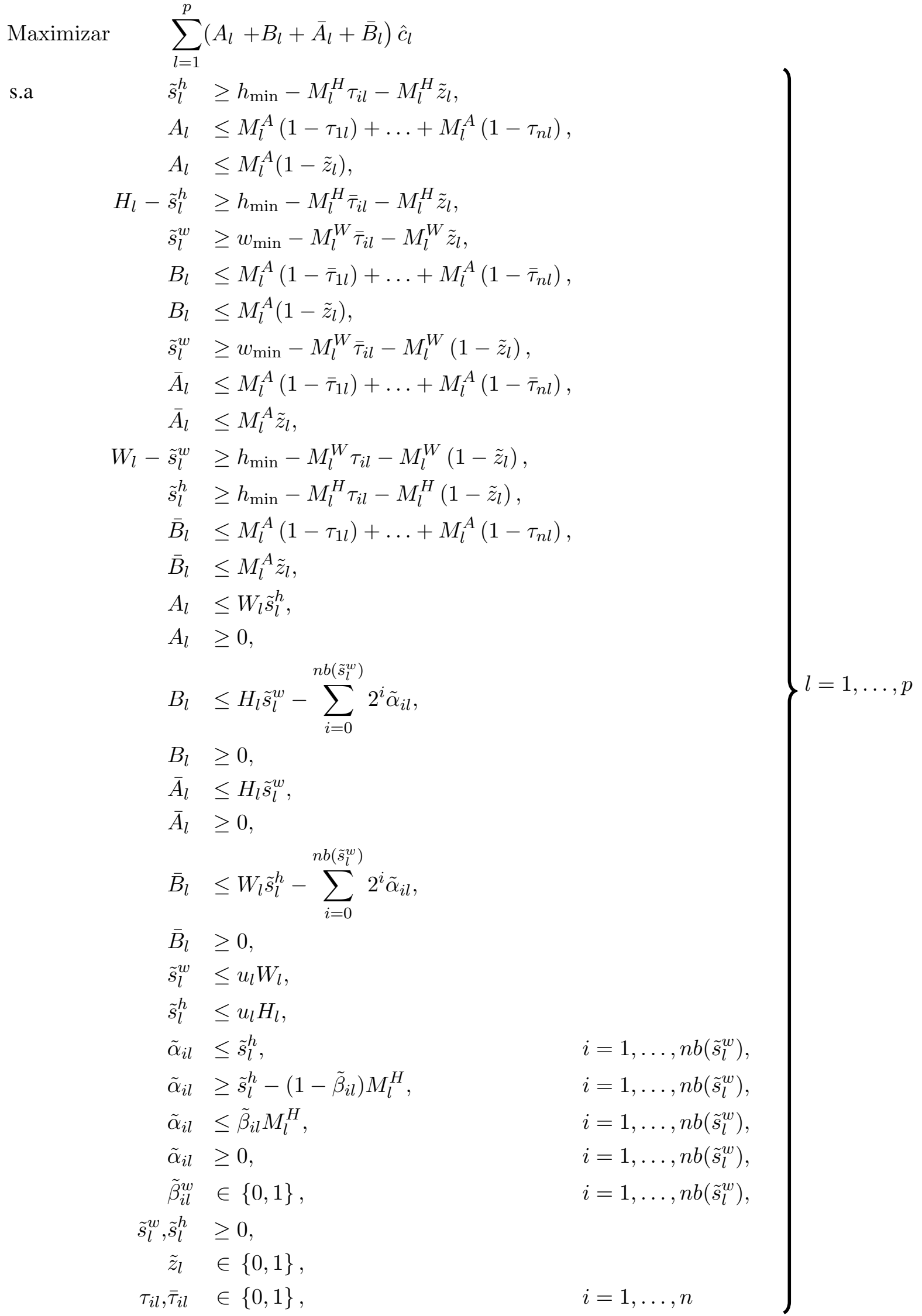




$$
\begin{aligned}
& \left(x, y, u, \bar{s}^{w}, \bar{s}^{h}\right) \in \operatorname{argmin} \sum_{l=1}^{p} u_{l} c_{l} \\
& \text { s.a } \frac{h_{i}+h_{k}}{2}-\left(y_{i}-y_{k}\right) \leq \eta_{i k} M_{l}^{H}+\bar{\eta}_{i k} M_{l}^{H} \\
& +\left(1-\bar{u}_{i l}\right) M_{l}^{H}+\left(1-\bar{u}_{k l}\right) M_{l}^{H}, \\
& i=1, \ldots, n, k=i+1, \ldots, n, l=1, \ldots, p \\
& \frac{h_{i}+h_{k}}{2}+\left(y_{i}-y_{k}\right) \leq\left(1-\eta_{i k}\right) M_{l}^{H}+\bar{\eta}_{i k} M_{l}^{H} \\
& +\left(1-\bar{u}_{i l}\right) M_{l}^{H}+\left(1-\bar{u}_{k l}\right) M_{l}^{H}, \\
& i=1, \ldots, n, k=i+1, \ldots, n, l=1, \ldots, p \\
& \frac{w_{i}+w_{k}}{2}-\left(x_{i}-x_{k}\right) \leq \eta_{i k} M_{l}^{W}+\left(1-\bar{\eta}_{i k}\right) M_{l}^{W} \\
& +\left(1-\bar{u}_{i l}\right) M_{l}^{W}+\left(1-\bar{u}_{k l}\right) M_{l}^{W}, \\
& i=1, \ldots, n, k=i+1, \ldots, n, l=1, \ldots, p \\
& \frac{w_{i}+w_{k}}{2}+\left(x_{i}-x_{k}\right) \leq\left(1-\eta_{i k}\right) M_{l}^{W}+\left(1-\bar{\eta}_{i k}\right) M_{l}^{W} \\
& +\left(1-\bar{u}_{i l}\right) M_{l}^{W}+\left(1-\bar{u}_{k l}\right) M_{l}^{W}, \\
& i=1, \ldots, n, k=i+1, \ldots, n, l=1, \ldots, p \\
& y_{i}-\frac{h_{i}}{2} \geq 0, i=1, \ldots, n, \\
& y_{i}+\frac{h_{i}}{2} \leq H_{l}-\tilde{s}_{l}^{h}+M_{l}^{h}\left(1-\bar{u}_{i l}\right), \\
& i=1, \ldots, n, l=1, \ldots, p \\
& x_{i}-\frac{w_{i}}{2} \geq 0, i=1, \ldots, n, \\
& x_{i}+\frac{w_{i}}{2} \leq W_{l}-\tilde{s}_{l}^{w}+M_{l}^{w}\left(1-\bar{u}_{i l}\right), \\
& i=1, \ldots, n, l=1, \ldots, p \\
& \bar{u}_{i l} \leq u_{l}, i=1, \ldots, n, l=1, \ldots, p, \\
& \sum_{l=1}^{p} \bar{u}_{i l} \leq 1, i=1, \ldots, n . \\
& u_{j} \in\{0,1\}, j=1, \ldots, p, \\
& \bar{u}_{i j} \in\{0,1\}, i=1, \ldots, n, j=1, \ldots, p, \\
& \eta_{i k}, \bar{\eta}_{i k} \in\{0,1\}, i=1, \ldots, n, k=i+1, \ldots, n \text {. }
\end{aligned}
$$

É importante observar que não substituímos $\tilde{s}_{l}^{w}$ por sua expansão binária de acordo com (4.72) ao escrever os modelos (4.115) e (4.116), a fim de simplificar a leitura dos modelos. Assim, onde há $\tilde{s}_{l}^{w}$, deve-se ler $\sum_{i=0}^{n b\left(\tilde{s}_{l}^{w}\right)} 2^{i} \tilde{\beta}_{i l}$.

Note que o modelo (4.115) pode ser reformulado em um MIP de forma similar ao modelo (3.12-3.26). Para isso, precisamos normalizar a função objetivo (4.114) para que assuma valores no intervalo $[0,1)$ e subtraí-la da função objetivo (4.14) da seguinte maneira:

$$
\sum_{l=1}^{p} u_{l} c_{l}-\frac{\sum_{l=1}^{p}\left(A_{l}+B_{l}+\bar{A}_{l}+\bar{B}_{l}\right) \hat{c}_{l}}{\sum_{l=1}^{p} W_{l} H_{l} \hat{c}_{l}} .
$$

Assim podemos definir a reformulação MIP de (4.115) como o problema de minimizar (4.117) sujeito às restrições do nível superior e inferior de (4.115), que será denominado $\mathcal{M}_{0}^{G}$. Similarmente, 
podemos definir a reformulação MIP de (4.116) como o problema de minimizar (4.117) sujeito às restrições do nível superior e inferior de (4.116), que será denominado $\mathcal{M}_{1}^{G}$. A Tabela 4.1 resume a quantidade de variáveis contínuas, binárias e a quantidade de restrições dos modelos $\mathcal{M}_{0}^{G}$ e $\mathcal{M}_{1}^{G}$. Notamos que a quantidade de variáveis reais é a mesma em ambos os modelos. A quantidade de variáveis binárias e de restrições são da mesma ordem de grandeza, embora o modelo $\mathcal{M}_{0}^{G}$ possua menos variáveis binárias e restrições que $\mathcal{M}_{1}^{G}$ para instâncias com $n>1$.

\begin{tabular}{|l|c|c|}
\hline & Modelo $\mathcal{M}_{0}^{G}$ & Modelo $\mathcal{M}_{1}^{G}$ \\
\hline Variáveis reais & $7 p+2 n+p n_{b}$ & $7 p+2 n+p n_{b}$ \\
Variáveis binárias & $4 p+n p+n^{2}-n+p n_{b}$ & $2 p+3 n p+n^{2}-n+p n_{b}$ \\
Restrições & $30 p+3 n p+2 n^{2} p+2 n+n^{2}+5 p n_{b}$ & $28 p+5 n p+2 n^{2} p+2 n+n^{2}+5 p n_{b}$ \\
\hline
\end{tabular}

Tabela 4.1: Comparação de $\mathcal{M}_{0}^{G}$ com $\mathcal{M}_{1}^{G}$ considerando a quantidade de variáveis e restrições de cada modelo. Consideramos $n_{b}=n b\left(\max _{l}\left(W_{l}, H_{l}\right)\right)$.

Apresentamos $\mathcal{M}_{0}^{G}$ e $\mathcal{M}_{1}^{G}$, que modelam o problema de corte no qual se deseja minimizar o custo dos objetos utilizados e maximizar o valor das sobras aproveitáveis (do tipo guilhotinado). A seguir apresentamos outros modelos considerando uma generalização das sobras do tipo guilhotinada.

\subsection{Considerando sobras aproveitáveis genéricas}

Nesta seção trabalhamos com sobras aproveitáveis genéricas, com o intuito de desenvolver modelos que possam contemplar um menor desperdício de material. Consideramos que cada objeto possui até duas sobras aproveitáveis e cada uma corresponde a uma região retangular, não necessariamente localizadas nas extremidades superior e direita do objeto. Da mesma forma que na seção anterior, definimos como aproveitável uma sobra quando (i) dado um par de dimensões mínimas $w_{\min } \times h_{\min }$, suas dimensões são maiores ou iguais a essas dimensões mínimas ou (ii) dada uma lista de dimensões de itens $w_{1} \times h_{1}, w_{2} \times h_{2}, \ldots, w_{m} \times h_{m}$, para algum $m \geq 1$, suas dimensões são maiores ou iguais às dimensões de pelo menos um par de dimensões da lista.

Para modelar as sobras, consideramos que cada objeto $l$ pode ter dois itens artificiais cujas dimensões horizontal e vertical são representadas, respectivamente, pelas variáveis $s_{l}^{w}, s_{l}^{h}$ e $t_{l}^{w}, t_{l}^{h}$. Sejam $w_{\min }$ e $h_{\min }$ as dimensões mínimas aceitáveis para que uma sobra seja considerada reaproveitável. Para cada objeto $l$, se $s_{l}^{w} \geq w_{\min }$ e $s_{l}^{h} \geq h_{\min }$, a sobra caracterizada pelas dimensões $s_{l}^{w}$ e $s_{l}^{h}$ é aproveitável. O mesmo vale para a sobras de dimensões $t_{l}^{w}$ e $t_{l}^{h}$. Denotamos por $\bar{a}\left(s_{l}^{w}, s_{l}^{h}\right) \mathrm{e}$ $\bar{a}\left(t_{l}^{w}, t_{l}^{h}\right)$ os valores das áreas dos itens caracterizados pelas dimensões $s_{l}^{w} \times s_{l}^{h}$ e $t_{l}^{w} \times t_{l}^{h}$, respectivamente. Definimos $\bar{a}\left(s_{l}^{w}, s_{l}^{h}\right)$ e $\bar{a}\left(t_{l}^{w}, t_{l}^{h}\right)$ de forma que correspondam à área de cada um dos itens artificiais, se e somente se ele for uma sobra aproveitável, ou seja:

$$
\bar{a}\left(\bar{w}_{l}, \bar{h}_{l}\right)= \begin{cases}\bar{w}_{l} \bar{h}_{l}, & \text { se } \bar{w}_{l} \geq w_{\min } \text { e } \bar{h}_{l} \geq h_{\mathrm{min}} \\ 0, & \text { caso contrário. }\end{cases}
$$

Assim, $s_{l}^{w}$ e $s_{l}^{h}$ são duas variáveis tais que, se ambas são maiores ou iguais que as dimensões mínimas correspondentes, então o seu produto representa o valor da sobra aproveitável. Caso contrário, pelo menos uma delas é menor do que uma das dimensões mínimas dadas e o valor da sobra correspondente deve ser zero.

Uma outra possibilidade seria pedir que os itens artificiais tenham dimensões maiores ou iguais às dimensões de pelo menos um dos itens que desejamos cortar, para serem considerados sobras reaproveitáveis. Ou seja, se $\left(s_{l}^{w} \geq w_{1}\right.$ e $\left.s_{l}^{h} \geq h_{1}\right)$ ou $\left(s_{l}^{w} \geq w_{2}\right.$ e $\left.s_{l}^{h} \geq h_{2}\right)$ ou ... ou $\left(s_{l}^{w} \geq\right.$ 
$w_{n}$ e $s_{l}^{h} \geq h_{n}$ ), então, o item artificial caracterizado pelas dimensões $s^{w} \times s^{h}$ é uma sobra aproveitável (o mesmo pode ser escrito para o item artificial caracterizado pelas dimensões $t^{w} \times t^{h}$ ). Dessa forma, podemos definir uma outra função que representará o valor da área das sobras da seguinte maneira:

$$
\overline{\bar{a}}\left(\bar{w}_{l}, \bar{h}_{l}\right)= \begin{cases}\bar{w}_{l} \bar{h}_{l}, & \text { se }\left(\bar{w}_{l} \geq w_{1} \text { e } \bar{h}_{l} \geq h_{1}\right) \text { ou } \ldots \text { ou }\left(\bar{w}_{l} \geq w_{n} \text { e } \bar{h}_{l} \geq h_{n}\right), \\ 0, & \text { caso contrário. }\end{cases}
$$

A fim de maximizar o valor das sobras aproveitáveis, considerando que as dimensões mínimas de uma sobra aproveitável são fornecidas, ou seja, optando pela definição (4.118), podemos definir a seguinte função objetivo a ser maximizada:

$$
\sum_{l=1}^{p}\left(\bar{a}\left(s_{l}^{w}, s_{l}^{h}\right)+\bar{a}\left(t_{l}^{w}, t_{l}^{h}\right)\right) \hat{c}_{l} .
$$

Caso optemos pela definição (4.119), a função objetivo a ser maximizada será:

$$
\sum_{l=1}^{p}\left(\overline{\bar{a}}\left(s_{l}^{w}, s_{l}^{h}\right)+\overline{\bar{a}}\left(t_{l}^{w}, t_{l}^{h}\right)\right) \hat{c}_{l} .
$$

A seguir, reformulamos (4.118) e (4.119) como restrições de um problema de otimização. Inicialmente apresentamos a reformulação de (4.118). Para tal, definimos a seguinte notação: $z_{l}, \bar{z}_{l}, \in\{0,1\}$, para $l=1, \ldots, p$ são variáveis artificiais; $A_{l}, B_{l} \in \mathbb{R}$ são variáveis que representam, respectivamente, $\bar{a}\left(s_{l}^{w}, s_{l}^{h}\right)$ e $\bar{a}\left(t_{l}^{w}, t_{l}^{h}\right) ; M_{l}^{A}, M_{l}^{W}$ e $M_{l}^{H}$, para $l=1, \ldots, p$, são constantes suficientemente grandes. Podemos reescrever (4.118) da seguinte maneira:

$$
\begin{aligned}
s_{l}^{w} & \geq w_{\min }-M_{l}^{W} z_{l}, \\
s_{l}^{h} & \geq h_{\min }-M_{l}^{H} z_{l}, \\
A_{l} & \leq s_{l}^{w} s_{l}^{h}, \\
A_{l} & \leq M_{l}^{A}\left(1-z_{l}\right), \\
t_{l}^{w} & \geq w_{\min }-M_{l}^{W} \bar{z}_{l}, \\
t_{l}^{h} & \geq h_{\min }-M_{l}^{H} \bar{z}_{l}, \\
B_{l} & \leq t_{l}^{w} t_{l}^{h}, \\
B_{l} & \leq M_{l}^{A}\left(1-\bar{z}_{l}\right), \\
s_{l}^{w}, s_{l}^{h}, t_{l}^{w}, t_{l}^{h}, A_{l}, B_{l} & \geq 0,
\end{aligned}
$$

para $l=1, \ldots, p$.

Se a variável $z_{l}$ receber o valor 0 , significa que (4.122), (4.123) e (4.124) estão sendo impostas pelo modelo, ou seja, temos que $s_{l}^{w} \geq w_{\min }$ e $s_{l}^{h} \geq h_{\min }, \operatorname{logo}, \bar{a}\left(s_{l}^{w}, s_{l}^{h}\right)=s_{l}^{w} s_{l}^{h}$. Note que ao maximizarmos o valor de $\sum_{l=1}^{p} A_{l}+B_{l}$ forçamos a desigualdade $A_{l} \leq s^{w} s_{l}^{h}$ a valer com igualdade. Se a variável $z_{l}$ receber o valor 1 , então a condição de que $\bar{a}\left(s_{l}^{w}, s_{l}^{h}\right)=0$ será imposta pelo modelo através da inequação (4.125). Vale mencionar que as desigualdades (4.126-4.129) são similares às desigualdades (4.122-4.125), porém relacionadas com a segunda sobra de cada placa, definida pelas dimensões $t_{l}^{h}$ e $t_{l}^{w}$. Em (4.130) temos a não negatividade das dimensões e dos valores das sobras.

Observando as desigualdades (4.124) e (4.128) notamos termos não-lineares. Desejamos eliminar essas não linearidades para construirmos modelos de programação inteira mista. Procedemos da mesma forma que fizemos com o termo $\tilde{s}_{l}^{h} \tilde{s}_{l}^{w}$ em (4.72). Podemos supor que na solução $s_{l}^{w}$ é um 
valor inteiro (uma vez que desejamos maximizar as sobras e as dimensões dos itens e dos objetos são todas inteiras), portanto podemos escrevê-lo como sua expansão binária:

$$
s_{l}^{w}=\sum_{i=0}^{n b\left(s_{l}^{w}\right)} 2^{i} \beta_{i l}, \quad \beta_{i l} \in\{0,1\} .
$$

Substituindo $s_{l}^{w}$ por (4.131) em (4.124) temos:

$$
A_{l} \leq\left(\sum_{i=0}^{\left(\left\lfloor\log _{2}\left(s_{l}^{w}\right)\right\rfloor+1\right)} 2^{i} \beta_{i l}\right) s_{l}^{h}
$$

ou equivalentemente:

$$
A_{l} \leq\left(\sum_{i=0}^{\left(\left\lfloor\log _{2}\left(s_{l}^{w}\right)\right\rfloor+1\right)} 2^{i} \alpha_{i l}\right)
$$

na qual substituímos $\beta_{i l} s_{l}^{h}$ por $\alpha_{i l}$ definido como:

$$
\alpha_{i l}= \begin{cases}s_{l}^{h}, & \text { se } \beta_{i l}=1, \\ 0, & \text { caso contrário. }\end{cases}
$$

Dessa forma (4.124) pode ser substituída por (4.132) mais as seguintes restrições:

$$
\begin{aligned}
\alpha_{i l} & \leq s_{l}^{h} \\
\alpha_{i l} & \geq s_{l}^{h}-M_{l}^{H}\left(1-\beta_{i l}\right) \\
\alpha_{i l} & \leq \beta_{i l} M_{l}^{H} \\
\alpha_{i l} & \geq 0
\end{aligned}
$$

para $i=1, \ldots, n b\left(s_{l}^{w}\right)$. Note que (4.134 - 4.137) é uma forma de modelar (4.133) com desigualdades lineares.

O raciocínio utilizado para linearizar (4.124) pode ser repetido a fim de linearizar (4.128). Utilizando $\delta_{i l}$ para a expansão binária de $t_{l}^{w}$, ou seja

$$
t_{l}^{w}=\sum_{i=0}^{n b\left(t_{l}^{w}\right)} 2^{i} \delta_{i l}, \quad \delta_{i l} \in\{0,1\}
$$

e definindo $\gamma_{i l}$ de forma similar a $\alpha_{i l}$, ou seja,

$$
\gamma_{i l}= \begin{cases}t_{l}^{h}, & \text { se } \delta_{i l}=1, \\ 0, & \text { caso contrário }\end{cases}
$$


podemos reescrever (4.128) da seguinte forma:

$$
\begin{aligned}
B_{l} & \leq\left(\sum_{i=0}^{n b\left(t_{l}^{w}\right)} 2^{i} \delta_{i l}\right)+M_{l}^{A} \bar{q}_{l} \\
\gamma_{i l} & \leq t_{l}^{h} \\
\gamma_{i l} & \geq t_{l}^{h}-M_{l}^{H}\left(1-\delta_{i l}\right), \\
\gamma_{i l} & \leq \delta_{i l} M_{l}^{H} \\
\gamma_{i l} & \geq 0 .
\end{aligned}
$$

De forma similar ao que fizemos com (4.118), podemos reformular (4.119) como restrições de um problema de otimização. Usamos essencialmente a mesma notação já introduzida em (4.1224.130), com exceção das novas variáveis $\tau_{i l}, \bar{\tau}_{i l}, \in\{0,1\}$, para $i=1, \ldots, n$ e $l=1, \ldots, p$ que são variáveis artificiais utilizadas para modelar a disjunção de (4.119):

$$
\begin{aligned}
& s_{l}^{w} \geq w_{i}-M_{l}^{W} \tau_{i l}, \quad \text { para } i=1, \ldots, n, \\
& s_{l}^{h} \geq h_{i}-M_{l}^{H} \tau_{i l}, \quad \quad \text { para } i=1, \ldots, n, \\
& A_{l} \leq s_{l}^{w} s_{l}^{h} \\
& A_{l} \leq M_{l}^{A}\left(1-\tau_{1 l}\right)+\ldots+M_{l}^{A}\left(1-\tau_{n l}\right), \\
& t_{l}^{w} \geq w_{i}-M_{l}^{W} \bar{\tau}_{i l} \\
& \text { para } i=1, \ldots, n, \\
& t_{l}^{h} \geq h_{i}-M_{l}^{H} \bar{\tau}_{i l}, \\
& \text { para } i=1, \ldots, n \text {, } \\
& B_{l} \leq t_{l}^{w} t_{l}^{h} \\
& B_{l} \leq M_{l}^{A}\left(1-\bar{\tau}_{1 l}\right)+\ldots+M_{l}^{A}\left(1-\bar{\tau}_{n l}\right), \\
& s_{l}^{w}, s_{l}^{h}, t_{l}^{w}, t_{l}^{h}, A_{l}, B_{l} \geq 0 \text {, }
\end{aligned}
$$

para $l=1 \ldots, p$.

As restrições (4.145), (4.146) e (4.147), juntamente com a maximização do valor das sobras impõem que se a sobra definida pelas dimensões $s_{l}^{h}$ e $s_{l}^{w}$ puder ser pelo menos do tamanho de um dos itens demandados, então será considerada uma sobra aproveitável. As restrições (4.148) e (4.153) impõem que, em caso contrário, o valor da sobra definida por $s_{l}^{h}$ e $s_{l}^{w}$ deve ser zero. As restrições (4.149-4.153) fazem o mesmo para as sobras referentes às dimensões $t_{l}^{h}$ e $t_{l}^{w}$. Da mesma forma como foi feito com (4.122-4.130), podemos reformular (4.145-4.153) de forma a eliminar as não linearidades, usando a expansão binária de $s_{l}^{w}$ e $t_{l}^{w}$.

Apresentamos a seguir modelos para o problema de corte com sobras aproveitáveis, considerando as sobras modeladas de acordo com (4.118) e (4.119). Desejamos obter uma solução que maximize o reaproveitamento de material dentre aquelas soluções que minimizam o custo relacionado às placas utilizadas, portanto, é natural escrever nosso problema como um problema de otimização em dois níveis. No nível superior desejamos maximizar o valor das sobras aproveitáveis e no nível inferior desejamos obter um padrão de corte que minimize o custo dos objetos.

Inicialmente apresentamos o modelo considerando as sobras modeladas de acordo com (4.118). Seja $p>0$ a quantidade de placas retangulares disponíveis; $W_{l}, H_{l}>0$ para $l=1, \ldots, p$ as dimensões horizontais e verticais de cada placa $l ; c_{l}$, uma constante que representa o custo da placa $l ; n>0$ a quantidade de itens que se deseja cortar; $w_{i}, h_{i}>0$ para $i=1, \ldots, n$ constantes que representam as dimensões horizontais e verticais de cada item $i ; x_{i}, y_{i}$ para $i=1, \ldots, n$ as variáveis que correspondem às coordenadas horizontal e vertical dos centros de cada item $i ; s_{l}^{w}, s_{l}^{h}$ e $t_{l}^{w}, t_{l}^{h}$ para $l=1, \ldots, p$, variáveis que representam as dimensões horizontais e verticais das duas sobras aproveitáveis que podem existir em cada placa $l ; x_{l}^{s}, y_{l}^{s}$ e $x_{l}^{t}, y_{l}^{t}$ para $l=1, \ldots, p$, variáveis que 
correspondem às coordenadas horizontais e verticais dos centros de cada uma das duas possíveis sobras aproveitáveis; $A_{l}, B_{l}, \operatorname{com} l=1, \ldots, p$ variáveis que representam os valores das possíveis sobras aproveitáveis para cada uma das placas; $u_{l} \in\{0,1\}$ para $l=1, \ldots, p$, variáveis que identificam se um objeto $l$ é utilizado ou não; $\bar{u}_{i l} \in\{0,1\}$ para $i=1, \ldots, n$ e $l=1, \ldots, p$, variáveis que identificam se o item $i$ é produzido a partir da placa $l ; z_{l}, \bar{z}_{l}, \in\{0,1\}$ para $l=1, \ldots, p$ variáveis que ajudam a modelar os aspectos condicionais das restrições e a relacioná-las com a função objetivo (no problema do nível superior); $\nu_{l}, \bar{\nu}_{l} \in\{0,1\}$ para $l=1, \ldots, p, \eta_{i l}, \bar{\eta}_{i l}, \in\{0,1\}$ para $i=1, \ldots, n$ e $l=1, \ldots, p$ e $v_{i}^{s}, v_{i}^{t}, \bar{v}_{i}^{s}, \bar{v}_{i}^{t} \in\{0,1\}$ para $i=1, \ldots, n$, variáveis que ajudam a modelar as disjunções (no nível inferior); $\alpha_{i l}, \gamma_{i l} \geq 0$ e $\beta_{i l}, \delta_{i l} \in\{0,1\}$, para $i=1, \ldots, n b\left(s_{l}^{w}\right)$ e $l=1, \ldots, p$, variáveis que ajudam a modelar a reformulação dos termos não lineares relativos à área das sobras; $M_{l}^{W}=W_{l}, M_{l}^{H}=H_{l}$ e $M_{l}^{A}=W_{l} H_{l}$, constantes utilizadas para modelar as disjunções. Podemos escrever o modelo de programação em dois níveis, considerando (4.118), da seguinte forma:

$$
\begin{aligned}
& \operatorname{Maximizar} \sum_{l=1}^{p}\left(A_{l}+B_{l}\right) \hat{c}_{l} \\
& \text { s.a } \\
& s_{l}^{w} \geq w_{\min }-M_{l}^{W} z_{l}, \\
& s_{l}^{h} \geq h_{\min }-M_{l}^{H} z_{l}, \\
& t_{l}^{w} \geq w_{\min }-M_{l}^{W} \bar{z}_{l}, \\
& t_{l}^{h} \geq h_{\min }-M_{l}^{H} \bar{z}_{l}, \\
& s_{l}^{w} \leq u_{l} W_{\max }, \\
& s_{l}^{h} \leq u_{l} H_{\max }, \\
& t_{l}^{w} \leq u_{l} W_{\max }, \\
& t_{l}^{h} \leq u_{l} H_{\max }, \\
& A_{l} \geq 0 \text {, } \\
& A_{l} \leq \sum_{i=0}^{n b\left(s_{l}^{w}\right)} 2^{i} \alpha_{i l}, \\
& A_{l} \leq M_{l}^{A}\left(1-z_{l}\right), \\
& \alpha_{i l} \leq s_{l}^{h}, \quad i=1, \ldots, n b\left(s_{l}^{w}\right), \\
& \alpha_{i l} \geq s_{l}^{h}-M_{l}^{H}\left(1-\beta_{i l}\right), \quad i=1, \ldots, n b\left(s_{l}^{w}\right), \\
& \alpha_{i l} \leq \beta_{i l} M_{l}^{H}, \quad i=1, \ldots, n b\left(s_{l}^{w}\right), \\
& \alpha_{i l} \geq 0 \quad i=1, \ldots, n b\left(s_{l}^{w}\right), \\
& B_{l} \geq 0 \text {, } \\
& B_{l} \leq \sum_{i=0}^{n b\left(t_{l}^{w}\right)} 2^{i} \gamma_{i l} \\
& B_{l} \leq M_{l}^{A}\left(1-\bar{z}_{l}\right), \\
& \gamma_{i l} \leq t_{l}^{h} \quad i=1, \ldots, n b\left(t_{l}^{w}\right), \\
& \gamma_{i l} \geq t_{l}^{h}-M_{l}^{H}\left(1-\delta_{i l}\right) \quad i=1, \ldots, n b\left(t_{l}^{w}\right), \\
& \gamma_{i l} \leq \delta_{i l} M_{l}^{H} \quad i=1, \ldots, n b\left(t_{l}^{w}\right), \\
& \gamma_{i l} \geq 0 \quad i=1, \ldots, n b\left(s_{l}^{w}\right), \\
& \beta_{i l}, \delta_{i l} \in\{0,1\}, \quad i=1, \ldots, n b\left(t_{l}^{w}\right), \\
& z_{l}, \bar{z}_{l}, \in\{0,1\}, \\
& \left(x, y, u, s^{w}, s^{h}, t^{w}, t^{h}\right) \in \arg \min \sum_{j=1}^{p} u_{j} c_{j}
\end{aligned}
$$

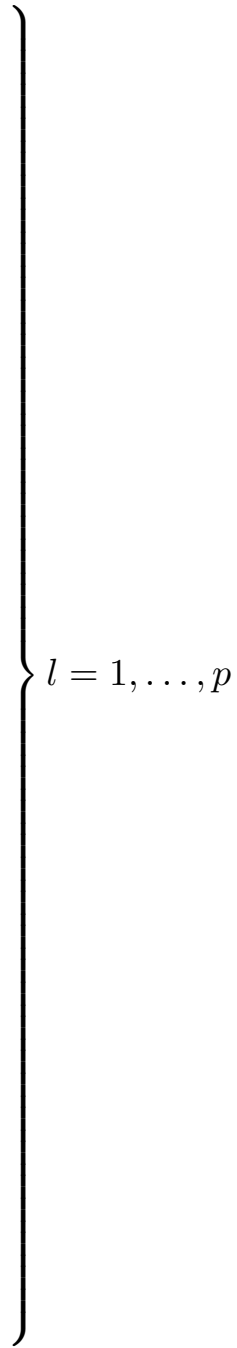




$$
\begin{aligned}
& \frac{s_{l}^{w}}{2}-x_{l}^{s} \leq 0 \\
& x_{l}^{s}+\frac{s_{l}^{w}}{2} \leq W_{l}+M_{l}^{W}\left(1-u_{l}\right), \\
& \frac{s_{l}^{h}}{2}-y_{l}^{s} \leq 0 \\
& y_{l}^{s}+\frac{s_{l}^{h}}{2} \leq H_{l}+M_{l}^{H}\left(1-u_{l}\right) \text {, } \\
& \frac{t_{l}^{w}}{2}-x_{l}^{t} \leq 0 \\
& x_{l}^{t}+\frac{t_{l}^{w}}{2} \leq W_{l}+M_{l}^{W}\left(1-u_{l}\right), \\
& \frac{t_{l}^{h}}{2}-y_{l}^{t} \leq 0 \\
& y_{l}^{t}+\frac{t_{l}^{h}}{2} \leq H_{l}+M_{l}^{H}\left(1-u_{l}\right), \\
& \frac{\left(s_{l}^{w}+t_{l}^{w}\right)}{2}-\left(x_{l}^{t}-x_{l}^{s}\right) \leq M^{A}\left(1-u_{l}\right)+M^{A} \nu_{l}+M^{A} \bar{\nu}_{l}, \\
& \frac{\left(s_{l}^{w}+t_{l}^{w}\right)}{2}+\left(x_{l}^{t}-x_{l}^{s}\right) \leq M^{A}\left(1-u_{l}\right)+M^{A}\left(1-\nu_{l}\right)+M^{A} \bar{\nu}_{l}, \\
& \frac{\left(s_{l}^{\left.h^{2}+t_{l}^{h}\right)}\right.}{2}-\left(y_{l}^{t}-y_{l}^{s}\right) \leq M^{A}\left(1-u_{l}\right)+M_{l}^{A}\left(1-\nu_{l}\right)+M^{A} \bar{\nu}_{l}, \\
& \frac{\left(s_{l}^{h}+t_{l}^{h}\right)}{2}+\left(y_{l}^{t}-y_{l}^{s}\right) \leq M^{A}\left(1-u_{l}\right)+M_{l}^{A}\left(1-\nu_{l}\right)+M^{A}\left(1-\bar{\nu}_{l}\right) \\
& \begin{array}{l}
\frac{w_{i}}{2}-x_{i} \leq 0 \\
\frac{h_{i}}{2}-y_{i} \leq 0
\end{array} \\
& \frac{h_{i}}{2}-y_{i} \leq 0 \\
& x_{i}+\frac{w_{i}}{2} \leq W_{l}+M_{l}^{W}\left(1-\bar{u}_{i l}\right), \\
& y_{i}+\frac{h_{i}}{2} \leq H_{l}+M_{l}^{W}\left(1-\bar{u}_{i l}\right), \\
& \frac{w_{i}+w_{k}}{2}-\left(x_{i}-x_{k}\right) \leq M_{l}^{W} \eta_{i k}+M_{l}^{W} \bar{\eta}_{i k}+ \\
& M_{l}^{W}\left(1-\bar{u}_{i l}\right)+M_{l}^{W}\left(1-\bar{u}_{k l}\right), \\
& \frac{w_{i}+w_{k}}{2}+\left(x_{i}-x_{k}\right) \leq M_{l}^{W}\left(1-\eta_{i k}\right)+M_{l}^{W} \bar{\eta}_{i k}+ \\
& M_{l}^{W}\left(1-\bar{u}_{i l}\right)+M_{l}^{W}\left(1-\bar{u}_{k l}\right), \\
& \frac{h_{i}+h_{k}}{2}-\left(y_{i}-y_{k}\right) \leq M_{l}^{H} \eta_{i k}+M_{l}^{H}\left(1-\bar{\eta}_{i k}\right)+ \\
& M_{l}^{H}\left(1-\bar{u}_{i l}\right)+M_{l}^{H}\left(1-\bar{u}_{k l}\right), \\
& \frac{h_{i}+h_{k}}{2}+\left(y_{i}-y_{k}\right) \leq M_{l}^{H}\left(1-\eta_{i k}\right)+M_{l}^{H}\left(1-\bar{\eta}_{i k}\right), \\
& M_{l}^{H}\left(1-\bar{u}_{i l}\right)+M_{l}^{H}\left(1-\bar{u}_{k l}\right), \\
& \frac{w_{i}+s_{l}^{w}}{2}-\left(x_{i}-x_{l}^{k}\right) \leq M_{l}^{W} v_{i}^{k}+M_{l}^{W} \bar{v}_{i}^{k}+ \\
& M_{l}^{W}\left(1-\bar{u}_{i l}\right) \text {, } \\
& \begin{aligned}
\frac{w_{i}+s_{l}^{w}}{2}+\left(x_{i}-x_{l}^{k}\right) \leq & M_{l}^{H}\left(1-v_{i}^{k}\right)+M_{l}^{H}\left(1-\bar{v}_{i}^{k}\right)+ \\
& M_{l}^{W}\left(1-\bar{u}_{i l}\right),
\end{aligned} \\
& \frac{h_{i}+s_{l}^{h}}{2}-\left(y_{i}-y_{l}^{k}\right) \leq M_{l}^{H} v_{i}^{k}+M_{l}^{H}\left(1-\bar{v}_{i}^{k}\right)+ \\
& M_{l}^{H}\left(1-\bar{u}_{i l}\right) \text {, } \\
& \begin{aligned}
\frac{h_{i}+s_{l}^{h}}{2}+\left(y_{i}-y_{l}^{k}\right) \leq & M_{l}^{W}\left(1-v_{i}^{k}\right)+M_{l}^{W} \bar{v}_{i}^{k}+ \\
& M_{l}^{H}\left(1-\bar{u}_{i l}\right)
\end{aligned} \\
& \begin{aligned}
\bar{u}_{i l} & \leq u_{l}, \\
\sum_{l=1}^{p} \bar{u}_{i l} & =1,
\end{aligned} \\
& u_{l}, \nu_{l}, \overline{\nu_{l}} \in\{0,1\} \text {, } \\
& \bar{u}_{i l} \in\{0,1\}, \\
& \eta_{i k}, \bar{\eta}_{i k} \in\{0,1\} \text {, } \\
& v_{i}, \bar{v}_{i} \in\{0,1\},
\end{aligned}
$$

$(4.154)$ 
Note que no problema do segundo nível, além das restrições de (4.14-4.27), há também restrições modelando a não sobreposição entre as sobras e os itens, em cada uma das placas.

Considerando as sobras modeladas de acordo com (4.119) e, adicionalmente, as variáveis $\tau_{i l}, \bar{\tau}_{i l}, \in\{0,1\}$, podemos escrever o modelo de otimização em dois níveis para o problema de corte com sobras aproveitáveis da seguinte forma:

$$
\begin{aligned}
& \text { Maximizar } \sum_{l=1}^{p}\left(A_{l}+B_{l}\right) \hat{c}_{l} \\
& \text { s.a } \\
& \begin{array}{rlr}
s_{l}^{w} \geq w_{i}-M_{l}^{W} \tau_{i l}, & i=1, \ldots, n, \\
s_{l}^{h} \geq h_{i}-M_{l}^{H} \tau_{i l}, & i=1, \ldots, n \\
t_{l}^{w} \geq w_{i}-M_{l}^{W} \bar{\tau}_{i l}, & i=1, \ldots, n \\
t_{l}^{h} \geq h_{i}-M_{l}^{H} \bar{\tau}_{i l}, & i=1, \ldots, n, \\
s_{l}^{w} \leq u_{l} W_{\max }, & \\
s_{l}^{h} \leq u_{l} H_{\max }, & \\
t_{l}^{w} & \leq u_{l} W_{\max } \\
t_{l}^{h} & \leq u_{l} H_{\max } \\
A_{l} & \geq 0
\end{array} \\
& A_{l} \leq \sum_{i=0}^{n b\left(s_{l}^{w}\right)} 2^{i} \alpha_{i l}, \\
& A_{l} \leq \sum_{i=1}^{n} M_{l}^{A}\left(1-\tau_{i l}\right), \\
& \alpha_{i l} \leq s_{l}^{h}, \quad i=1, \ldots, n b\left(s_{l}^{w}\right), \\
& \alpha_{i l} \geq s_{l}^{h}-M_{l}^{H}\left(1-\beta_{i l}\right), \quad i=1, \ldots, n b\left(s_{l}^{w}\right), \\
& \alpha_{i l} \leq \beta_{i l} M_{l}^{H}, \quad i=1, \ldots, n b\left(s_{l}^{w}\right), \\
& \alpha_{i l} \geq 0 \quad i=1, \ldots, n b\left(s_{l}^{w}\right), \\
& B_{l} \geq 0 \\
& B_{l} \leq \sum_{i=0}^{n b\left(s_{l}^{w}\right)} 2^{i} \gamma_{i l}, \\
& B_{l} \leq \sum_{i=1}^{n} M_{l}^{A}\left(1-\bar{\tau}_{i l}\right), \\
& \gamma_{i l} \leq t_{l}^{h} \quad i=1, \ldots, n b\left(s_{l}^{w}\right), \\
& \gamma_{i l} \geq t_{l}^{h}-M_{l}^{H}\left(1-\delta_{i l}\right) \quad i=1, \ldots, n b\left(s_{l}^{w}\right), \\
& \gamma_{i l} \leq \delta_{i l} M_{l}^{H} \quad i=1, \ldots, n b\left(s_{l}^{w}\right), \\
& \gamma_{i l} \geq 0 \quad i=1, \ldots, n b\left(s_{l}^{w}\right), \\
& \beta_{i l}, \delta_{i l} \in\{0,1\}, \quad i=1, \ldots, n b\left(t_{l}^{w}\right), \\
& \tau_{i l}, \bar{\tau}_{i l}, \in\{0,1\}, \quad i=1, \ldots, n, \\
& \left(x, y, u, s^{w}, s^{h}, t^{w}, t^{h}\right) \in \arg \min \sum_{l=1}^{p} u_{l} v_{l}
\end{aligned}
$$

s.a

$$
\begin{array}{rlrl}
\frac{s_{l}^{w}}{2}-x_{l}^{s} \leq 0, & & =1, \ldots, p, \\
x_{l}^{s}+\frac{s_{l}^{w}}{2} \leq W_{l}+M_{l}^{W}\left(1-u_{l}\right), & l=1, \ldots, p, \\
\frac{s_{l}^{h}}{2}-y_{l}^{s} \leq 0, & & l=1, \ldots, p, \\
y_{l}^{s}+\frac{s_{l}^{h}}{2} \leq H_{l}+M_{l}^{H}\left(1-u_{l}\right), & & l=1, \ldots, p, \\
\frac{t_{l}^{w}}{2}-x_{l}^{t} \leq 0, & & l=1, \ldots, p, \\
x_{l}^{t}+\frac{t_{l}^{w}}{2} \leq W_{l}+M_{l}^{W}\left(1-u_{l}\right), & l=1, \ldots, p, \\
\frac{t_{l}^{h}}{2}-y_{l}^{t} \leq 0, & & l=1, \ldots, p, \\
y_{l}^{t}+\frac{t_{l}^{h}}{2} \leq H_{l}+M_{l}^{H}\left(1-u_{l}\right), & & l=1, \ldots, p,
\end{array}
$$




$$
\begin{aligned}
& \frac{\left(s_{l}^{w}+t_{l}^{w}\right)}{\left(s^{2} t^{w}\right)}-\left(x_{l}^{t}-x_{l}^{s}\right) \leq M_{l}^{A}\left(1-u_{l}\right)+M_{l}^{A} \nu_{l}+M_{l}^{A} \bar{\nu}_{l}, \\
& l=1, \ldots, p, \\
& \frac{\left(s_{l}^{w}+t_{l}^{w}\right)}{2}+\left(x_{l}^{t}-x_{l}^{s}\right) \leq M_{l}^{A}\left(1-u_{l}\right)+M_{l}^{A}\left(1-\nu_{l}\right)+M_{l}^{A} \bar{\nu}_{l}, \\
& l=1, \ldots, p, \\
& \frac{\left(s_{l}^{\left.h^{2}+t_{l}^{h}\right)}\right.}{2}-\left(y_{l}^{t}-y_{l}^{s}\right) \leq M_{l}^{A}\left(1-u_{l}\right)+M_{l}^{A}\left(1-\nu_{l}\right)+M_{l}^{A} \bar{\nu}_{l}, \\
& l=1, \ldots, p, \\
& \frac{\left(s_{l}^{h}+t_{l}^{h}\right)}{2}+\left(y_{l}^{t}-y_{l}^{s}\right) \leq \\
& \begin{array}{l}
\frac{w_{i}}{2}-x_{i} \leq 0, \\
\frac{h_{i}}{2}-y_{i} \leq 0,
\end{array} \\
& \frac{h_{i}}{2}-y_{i} \leq 0 \\
& x_{i}+\frac{w_{i}}{2} \leq W_{l}+M_{l}^{W}\left(1-\bar{u}_{i l}\right) \text {, } \\
& y_{i}+\frac{h_{i}}{2} \leq H_{l}+M_{l}^{W}\left(1-\bar{u}_{i l}\right), \\
& \frac{w_{i}+w_{k}}{2}-\left(x_{i}-x_{k}\right) \leq M_{l}^{W} \eta_{i k}+M_{l}^{W} \bar{\eta}_{i k}+ \\
& M_{l}^{W}\left(1-\bar{u}_{i l}\right)+M_{l}^{W}\left(1-\bar{u}_{k l}\right), \\
& \frac{w_{i}+w_{k}}{2}+\left(x_{i}-x_{k}\right) \leq M_{l}^{W}\left(1-\eta_{i k}\right)+M_{l}^{W} \bar{\eta}_{i k}+ \\
& M_{l}^{W}\left(1-\bar{u}_{i l}\right)+M_{l}^{W}\left(1-\bar{u}_{k l}\right), \\
& \frac{h_{i}+h_{k}}{2}-\left(y_{i}-y_{k}\right) \leq M_{l}^{H} \eta_{i k}+M_{l}^{H}\left(1-\bar{\eta}_{i k}\right)+ \\
& M_{l}^{H}\left(1-\bar{u}_{i l}\right)+M_{l}^{H}\left(1-\bar{u}_{k l}\right), \\
& \frac{h_{i}+h_{k}}{2}+\left(y_{i}-y_{k}\right) \leq M_{l}^{H}\left(1-\eta_{i k}\right)+M_{l}^{H}\left(1-\bar{\eta}_{i k}\right), \\
& M_{l}^{H}\left(1-\bar{u}_{i l}\right)+M_{l}^{H}\left(1-\bar{u}_{k l}\right), \\
& \frac{w_{i}+s_{l}^{w}}{2}-\left(x_{i}-x_{l}^{k}\right) \leq M_{l}^{W} v_{i}^{k}+M_{l}^{W} \bar{v}_{i}^{k}+ \\
& M_{l}^{W}\left(1-\bar{u}_{i l}\right) \text {, } \\
& \begin{aligned}
\frac{w_{i}+s_{l}^{w}}{2}+\left(x_{i}-x_{l}^{k}\right) \leq & M_{l}^{H}\left(1-v_{i}^{k}\right)+M_{l}^{H}\left(1-\bar{v}_{i}^{k}\right)+ \\
& M_{l}^{W}\left(1-\bar{u}_{i l}\right),
\end{aligned} \\
& \frac{h_{i}+s_{l}^{h}}{2}-\left(y_{i}-y_{l}^{k}\right) \leq M_{l}^{H} v_{i}^{k}+M_{l}^{H}\left(1-\bar{v}_{i}^{k}\right)+ \\
& M_{l}^{H}\left(1-\bar{u}_{i l}\right) \text {, } \\
& \begin{aligned}
\frac{h_{i}+s_{l}^{h}}{2}+\left(y_{i}-y_{l}^{k}\right) \leq & M_{l}^{W}\left(1-v_{i}^{k}\right)+M_{l}^{W} \bar{v}_{i}^{k}+ \\
& M_{l}^{H}\left(1-\bar{u}_{i l}\right)
\end{aligned} \\
& \begin{aligned}
\bar{u}_{i l} & \leq u_{l}, \\
\sum_{l=1}^{p} \bar{u}_{i l} & =1, \\
u_{l}, \nu_{l}, \bar{\nu}_{l} & \in\{0,1\}, \\
\bar{u}_{i l} & \in\{0,1\}, \\
\eta_{i k}, \bar{\eta}_{i k} & \in\{0,1\}, \\
v_{i}, \bar{v}_{i} & \in\{0,1\},
\end{aligned} \\
& l=1, \ldots, p, \\
& i=1, \ldots, n, \\
& i=1, \ldots, n \text {, } \\
& i=1, \ldots, n, l=1, \ldots, p, \\
& i=1, \ldots, n, l=1, \ldots, p, \\
& i=1, \ldots, n-1, k=i+1, \ldots, n, \\
& l=1, \ldots, p, \\
& i=1, \ldots, n-1, k=i+1, \ldots, n, \\
& l=1, \ldots, p, \\
& i=1, \ldots, n-1, k=i+1, \ldots, n, \\
& l=1, \ldots, p, \\
& i=1, \ldots, n-1, k=i+1, \ldots, n, \\
& l=1, \ldots, p, \\
& i=1, \ldots, n, k=\{s, t\}, \\
& l=1, \ldots, p, \\
& i=1, \ldots, n, k=\{s, t\}, \\
& l=1, \ldots, p, \\
& i=1, \ldots, n, k=\{s, t\}, \\
& l=1, \ldots, p, \\
& i=1, \ldots, n, k=\{s, t\}, \\
& l=1, \ldots, p, \\
& i=1, \ldots, n, l=1, \ldots, p, \\
& i=1, \ldots, n \\
& l=1, \ldots, p, \\
& i=1, \ldots, n, l=1, \ldots, p \\
& i=1, \ldots, n, k=i+1, \ldots, n \text {, } \\
& i=1, \ldots, n \text {. }
\end{aligned}
$$

É importante notar que não substituímos as variáveis $s_{l}^{w}$ e $t_{l}^{w}$ de acordo com (4.131) e (4.138), respectivamente, ao escrever os modelos (4.154) e (4.155), a fim de simplificar a leitura do modelo. Assim, onde há $s_{l}^{w}$, deve-se ler $\sum_{i=0}^{n b\left(s_{l}^{w}\right)} 2^{i} \beta_{i l}$ e onde há $t_{l}^{w}$, deve-se ler $\sum_{i=0}^{n b\left(t_{l}^{w}\right)} 2^{i} \delta_{i l}$.

Tanto o problema (4.154) como o problema (4.155) podem ser reformulados como um modelo de programação inteira mista, para isso basta utilizarmos a mesma técnica utilizada na Seção 4.2. Esta técnica consiste em montar um modelo de programação inteira mista cujas restrições correspondem à união das restrições do problema do nível superior e inferior do problema de 
otimização em dois níveis, em questão. A função objetivo consiste na função objetivo do problema do nível superior normalizada, de forma a assumir valores no intervalo $[0,1)$, subtraída da função objetivo do problema do nível inferior. Dessa forma, a função objetivo de nosso MIP para qualquer um dos modelos (4.154) ou (4.155) pode ser escrita da seguinte forma:

$$
\sum_{l=1}^{p} u_{l} v_{l}-\frac{\sum_{l=1}^{p}\left(A_{l}+B_{l}\right) \hat{c}_{l}}{\sum_{l=1}^{p} W_{l} H_{l} \hat{c}_{l}} .
$$

Chamamos de $\mathcal{M}_{2}^{G}$ o modelo MIP formado pela minimização de (4.156) mais as restrições dos níveis superior e inferior de (4.154), enquanto que chamamos de $\mathcal{M}_{3}^{G}$ o modelo MIP formado pela minimização de (4.156) mais as restrições dos níveis superior e inferior de (4.155). A Tabela 4.2 apresenta um resumo da quantidade de restrições e de variáveis reais e binárias dos modelos $\mathcal{M}_{2}^{G}$ e $\mathcal{M}_{3}^{G}$. Notamos que a quantidade de variáveis reais é a mesma em ambos os modelos. A quantidade de variáveis binárias e de restrições são da mesma ordem de grandeza, embora o modelo $\mathcal{M}_{2}^{G}$ possua menos variáveis binárias e restrições que $\mathcal{M}_{3}^{G}$ para instâncias com $n>1$. Comparando a Tabela 4.2 com a Tabela 4.1, observamos que os modelos $\mathcal{M}_{0}^{G}$ e $\mathcal{M}_{1}^{G}$ possuem uma menor quantidade de variáveis e restrições que os modelos $\mathcal{M}_{2}^{G}$ e $\mathcal{M}_{3}^{G}$.

\begin{tabular}{|l|c|c|}
\hline & Modelo $\mathcal{M}_{2}^{G}$ & Modelo $\mathcal{M}_{3}^{G}$ \\
\hline Variáveis reais & $8 p+2 n+2 p n_{b}$ & $8 p+2 n+2 p n_{b}$ \\
Variáveis binárias & $5 p+n p+n^{2}+n+2 p n_{b}$ & $3 p+3 n p+n^{2}+n+2 p n_{b}$ \\
Restrições & $31 p+10 n p+2 n^{2} p+4 n+n^{2}+10 p n_{b}$ & $25 p+16 n p+2 n^{2} p+4 n+n^{2}+10 p n_{b}$ \\
\hline
\end{tabular}

Tabela 4.2: Comparação de $\mathcal{M}_{2}^{G}$ com $\mathcal{M}_{3}^{G}$ considerando a quantidade de variáveis e restrições de cada modelo. Consideramos $n_{b}=n b\left(\max _{l}\left(W_{l}, H_{l}\right)\right)$.

\subsection{Experimentos Numéricos}

Nesta seção apresentamos alguns resultados obtidos a partir de experimentos numéricos com os modelos apresentados. A Tabela 4.3 descreve as instâncias utilizadas nos experimentos. Na primeira coluna temos o número que identifica a instância, na segunda e terceira colunas temos a quantidade de itens e a quantidade de tipos distintos de itens, respectivamente, na terceira e quarta coluna temos a quantidade de objetos e a quantidade de tipos distintos de objetos, respectivamente. Abaixo das quantidades, temos as dimensões dos diferentes tipos de itens ou objetos no formato $N(W \times H)$, no qual $N$ refere-se à quantidade itens ou objetos daquele tipo e $W \times H$ refere-se às suas dimensões horizontal e vertical, respectivamente. Tais instâncias foram geradas através do mesmo gerador apresentado na Seção 3.4. Nestas instâncias consideramos como custo dos objetos sua área.

\begin{tabular}{|c||c|c|c|c|}
\hline \multicolumn{4}{|c|}{ Descrição das Instâncias } \\
\hline \multirow{2}{*}{$\#$} & \multicolumn{2}{|c|}{ Itens } & \multicolumn{2}{c|}{ Objetos } \\
\cline { 2 - 5 } & Qtde. Total & Qtde. Tipos & Qtde. Total & Qtde. Tipos \\
\hline \multirow{3}{*}{1} & 5 & 2 & 2 & 2 \\
\cline { 2 - 5 } & \multicolumn{2}{|c|}{$3(2 \times 11)$} & \multicolumn{2}{|c|}{$1(22 \times 17)$} \\
& \multicolumn{2}{|c|}{$2(5 \times 5)$} & \multicolumn{2}{c|}{$1(14 \times 30)$} \\
\hline \multicolumn{4}{|c|}{ Continua na próxima página } \\
\hline
\end{tabular}


Tabela 4.3 - continuação da página anterior

\begin{tabular}{|c|c|c|c|c|}
\hline \multirow{2}{*}{ \# } & \multicolumn{2}{|c|}{ Itens } & \multicolumn{2}{|c|}{ Objetos } \\
\hline & Qtde. Total & Qtde. Tipos & Qtde. Total & Qtde. Tipos \\
\hline \multirow[b]{2}{*}{2} & 2 & 1 & 2 & 2 \\
\hline & \multicolumn{2}{|c|}{$2(2 \times 4)$} & \multicolumn{2}{|c|}{$\begin{array}{l}1(10 \times 17) \\
1(10 \times 24)\end{array}$} \\
\hline \multirow[b]{2}{*}{3} & 3 & 1 & 2 & 2 \\
\hline & \multicolumn{2}{|c|}{$3(5 \times 4)$} & \multicolumn{2}{|c|}{$\begin{array}{l}1(18 \times 19) \\
1(26 \times 22)\end{array}$} \\
\hline \multirow[b]{2}{*}{4} & 7 & 3 & 3 & 3 \\
\hline & \multicolumn{2}{|c|}{$\begin{array}{l}4(3 \times 3) \\
1(4 \times 3) \\
2(7 \times 1)\end{array}$} & \multicolumn{2}{|c|}{$\begin{array}{l}1(24 \times 12) \\
1(15 \times 18) \\
1(17 \times 13)\end{array}$} \\
\hline \multirow[b]{2}{*}{5} & 5 & 2 & 2 & 2 \\
\hline & \multicolumn{2}{|c|}{$\begin{array}{c}4(7 \times 1) \\
1(11 \times 1)\end{array}$} & \multicolumn{2}{|c|}{$\begin{array}{l}1(20 \times 10) \\
1(29 \times 12)\end{array}$} \\
\hline \multirow[b]{2}{*}{6} & 7 & 3 & 2 & 2 \\
\hline & \multicolumn{2}{|c|}{$\begin{array}{c}2(11 \times 11) \\
3(2 \times 11) \\
2(5 \times 5)\end{array}$} & \multicolumn{2}{|c|}{$\begin{array}{l}1(22 \times 17) \\
1(14 \times 30)\end{array}$} \\
\hline \multirow[b]{2}{*}{7} & 9 & 2 & 5 & 3 \\
\hline & \multicolumn{2}{|c|}{$\begin{array}{l}5(9 \times 6) \\
4(5 \times 3)\end{array}$} & \multicolumn{2}{|c|}{$\begin{array}{l}1(27 \times 23) \\
2(19 \times 17) \\
2(19 \times 19)\end{array}$} \\
\hline \multirow[b]{2}{*}{8} & 9 & 2 & 2 & 2 \\
\hline & \multicolumn{2}{|c|}{$\begin{array}{l}5(3 \times 2) \\
4(3 \times 1)\end{array}$} & \multicolumn{2}{|c|}{$\begin{array}{l}1(19 \times 17) \\
1(16 \times 11)\end{array}$} \\
\hline \multirow[b]{2}{*}{9} & 10 & 2 & 2 & 2 \\
\hline & \multicolumn{2}{|c|}{$\begin{array}{l}4(3 \times 4) \\
1(3 \times 1)\end{array}$} & \multicolumn{2}{|c|}{$\begin{array}{l}1(18 \times 20) \\
1(13 \times 10)\end{array}$} \\
\hline \multirow[b]{2}{*}{10} & 10 & 2 & 2 & 2 \\
\hline & \multicolumn{2}{|c|}{$\begin{array}{l}7(4 \times 5) \\
3(5 \times 2)\end{array}$} & \multicolumn{2}{|c|}{$\begin{array}{l}1(22 \times 14) \\
1(18 \times 22)\end{array}$} \\
\hline
\end{tabular}

Tabela 4.3: Descrição das instâncias $1,2, \ldots, 9$.

Os modelos foram implementados utilizando o CPLEX 12.1 e a Concert Techonology 2.9. Com relação às opções de configuração do CPLEX, definimos a variável PreInd, referente ao presolve, como false e mudamos a variável EpGap, referente à precisão relativa exigida pelo solver para considerar um ponto viável como solução, para $10^{-7}$ (o valor padrão é $10^{-4}$ ). Alteramos a variável Pre Ind com o intuito de avaliar os modelos de forma justa, já que, quando ativado o presolve, o CPLEX efetua manipulações em cada modelo a fim de acelerar a posterior resolução. Alteramos a variável EpGap com o intuito de evitar que o CPLEX considere como solução um ponto que não corresponde a uma solução real, conforme já explicado na Seção 3.4. Também modificamos o tempo limite para resolução de uma instância para $6 \mathrm{~h}$ (definimos o parâmetro Ti Lim como 21600) e pedimos para que o CPLEX armazene a árvore Branch\&Bound em disco caso ela exceda 5000MB (definimos NodeFileInd como 3 e WorkMem como 5000). Os programas foram compilados utilizando o compilador G++ 4.4.3 da GCC (GNU Compiller Collection) e os experimentos realizados em uma máquina com $8 \mathrm{~GB}$ de memória RAM, dois processadores de 2.6 
GHz Intel Xeon, com 6 núcleos cada e tecnologia Hyper Threading. Nos modelos $\mathcal{M}_{0}^{G}$ e $\mathcal{M}_{2}^{G}$ consideramos como dimensões mínimas das sobras aproveitáveis $w_{\min }=3$ e $h_{\min }=3$. Nos modelos $\mathcal{M}_{1}^{G}$ e $\mathcal{M}_{3}^{G}$, consideramos como dicionário de itens a lista de itens a serem cortados. Em todos os experimentos consideramos $M_{l}^{A}=\max \left\{W_{l} H_{l}: l=1, \ldots, p\right), M_{l}^{W}=\max \left\{W_{l}: l=1, \ldots, p\right)$ e $M_{l}^{H}=\max \left\{H_{l}: l=1, \ldots, p\right)$ para $l=1, \ldots, p$.

\begin{tabular}{|c|c|c|c|c|c|c|c|}
\hline \multicolumn{8}{|c|}{ Modelo $\mathcal{M}_{0}^{G}$} \\
\hline \multirow[t]{2}{*}{ \# Instância } & \multicolumn{4}{|c|}{ Dados das soluções } & \multicolumn{3}{|c|}{ Medidas de desempenho } \\
\hline & Lim. Inf. & Lim. Sup. & Valor das Sobras & Placas Usadas & Iterações MIP & Nós B\&B & Tempo (s) \\
\hline 1 & 373,700824596234 & 373,700824596234 & 94622 & 1 & 1742 & 788 & 0,23 \\
\hline 2 & 239,872276308918 & 239,872276308918 & 38400 & 1 & 66 & 3 & 0,07 \\
\hline 3 & 341,782856165062 & 341,782856165062 & 96444 & 1 & 369 & 168 & 0,13 \\
\hline 4 & 220,826167037155 & 220,826167037155 & 35581 & 1 & 406396 & 208699 & 15,26 \\
\hline 5 & 219,768973003587 & 219,768973003587 & 39160 & 1 & 5004 & 2713 & 0,38 \\
\hline 6 & 793,446789934530 & 793,446850219429 & 174948 & 2 & 158124313 & 55123723 & 3083,38 \\
\hline 7 & 323,000000000000 & 361,000000000000 & - & - & - & - & $\geq 21600,00$ \\
\hline 8 & 175,817892908614 & 175,825697498245 & - & - & - & - & $\geq 21600,00$ \\
\hline 9 & 129,924573378840 & 129,937883959044 & - & - & - & - & $\geq 21600,00$ \\
\hline 10 & 307,798076923077 & 307,851923076923 & - & - & - & - & $\geq 21600,00$ \\
\hline \multicolumn{8}{|c|}{ Modelo $\mathcal{M}_{1}^{G}$} \\
\hline \multirow[t]{2}{*}{ \# Instância } & \multicolumn{4}{|c|}{ Dados das soluções } & \multicolumn{3}{|c|}{ Medidas de desempenho } \\
\hline & Lim. Inf. & Lim. Sup. & Valor das Sobras & Placas Usadas & Iterações MIP & Nós B\&B & Tempo (s) \\
\hline 1 & 373,700824596230 & 373,700824596230 & 94622 & 1 & 1264 & 620 & 0,23 \\
\hline 2 & 239,872276308918 & 239,872276308918 & 38400 & 1 & 94 & 2 & 0,09 \\
\hline 3 & 341,782856165062 & 341,782856165062 & 96444 & 1 & 411 & 151 & 0,14 \\
\hline 4 & 220,826167037155 & 220,826167037155 & 35581 & 1 & 595343 & 306489 & 22,16 \\
\hline 5 & 219,768973003587 & 219,768973003587 & 39160 & 1 & 5675 & 2139 & 0,38 \\
\hline 6 & 793,446780659930 & 793,446850219429 & 174948 & 2 & 185616149 & 63858885 & 4038,32 \\
\hline 7 & 361,000000000000 & 361,000000000000 & 854941 & 1 & 1068376 & 158691 & 18,69 \\
\hline 8 & 175,817892908614 & 175,825692888509 & - & - & - & - & $\geq 21600,00$ \\
\hline 9 & 129,932559726962 & 129,932559726962 & 9880 & 1 & 1792070 & 722791 & 54,78 \\
\hline 10 & 307,782167832168 & 307,851923076923 & - & - & - & - & $\geq 21600,00$ \\
\hline \multicolumn{8}{|c|}{ Modelo $\mathcal{M}_{2}^{G}$} \\
\hline \multirow[t]{2}{*}{ \# Instância } & \multicolumn{4}{|c|}{ Dados das soluções } & \multicolumn{3}{|c|}{ Medidas de desempenho } \\
\hline & Lim. Inf. & Lim. Sup. & Valor das Sobras & Placas Usadas & Iterações MIP & Nós B\&B & Tempo (s) \\
\hline 1 & 373,700824596226 & 373,700824596226 & 94622 & 1 & 4565121 & 997992 & 73,13 \\
\hline 2 & 239,872276308918 & 239,872276308918 & 38400 & 1 & 2180 & 424 & 0,15 \\
\hline 3 & 341,782856165058 & 341,782856165058 & 96444 & 1 & 88855 & 23165 & 1,98 \\
\hline 4 & 220,823986250857 & 220,824007621449 & 36023 & 1 & 366404932 & 96722280 & 8939,51 \\
\hline 5 & 219,766377194610 & 219,766377194610 & 39600 & 1 & 12459369 & 2938239 & 247,92 \\
\hline 6 & 792,154137525452 & 793,444763434469 & - & - & - & - & $\geq 21600,00$ \\
\hline 7 & 323,000000000000 & 361,000000000000 & - & - & - & - & $\geq 21600,00$ \\
\hline 8 & 175,802283729352 & 175,825697498241 & - & - & - & - & $\geq 21600,00$ \\
\hline 9 & 129,911262798624 & 129,932559726957 & - & - & - & - & $\geq 21600,00$ \\
\hline 10 & 307,647552447552 & 307,836013986003 & - & - & - & - & $\geq 21600,00$ \\
\hline \multicolumn{8}{|c|}{ Modelo $\mathcal{M}_{3}^{G}$} \\
\hline \multirow[t]{2}{*}{ \# Instância } & \multicolumn{4}{|c|}{ Dados das soluções } & \multicolumn{3}{|c|}{ Medidas de desempenho } \\
\hline & Lim. Inf. & Lim. Sup. & Valor das Sobras & Placas Usadas & Iterações MIP & Nós B\&B & Tempo (s) \\
\hline 1 & 373,700824596239 & 373,700824596239 & 94622 & 1 & 3591567 & 910136 & 66,24 \\
\hline 2 & 239,872276308918 & 239,872276308918 & 38400 & 1 & 3171 & 931 & 0,17 \\
\hline 3 & 341,782856165058 & 341,782856165058 & 96444 & 1 & 26503 & 8365 & 0,84 \\
\hline 4 & 220,823988167271 & 220,824007621461 & 36023 & 1 & 665811114 & 146884889 & 14525,18 \\
\hline 5 & 219,768959177707 & 219,768973003587 & 39160 & 1 & 20821821 & 4754522 & 399,06 \\
\hline 6 & 419,703866243408 & 793,444763434469 & - & - & - & - & $\geq 21600,00$ \\
\hline 7 & 322,945218441974 & 361,000000000000 & - & - & - & - & $\geq 21600,00$ \\
\hline 8 & 175,802283729344 & 175,825697461551 & - & - & - & - & $\geq 21600,00$ \\
\hline 9 & 129,920136518768 & 129,932559726957 & - & - & - & - & $\geq 21600,00$ \\
\hline 10 & 307,714860139842 & 307,833566433549 & - & - & - & - & $\geq 21600,00$ \\
\hline
\end{tabular}

Tabela 4.4: Resultados dos experimentos com os modelos $\mathcal{M}_{0}^{G}, \mathcal{M}_{1}^{G}, \mathcal{M}_{2}^{G}$ e $\mathcal{M}_{3}^{G}$.

O resultado dos experimentos podem ser vistos na Tabela 4.4. Para cada instância do problema de corte e cada modelo, informamos na coluna "Lim. Inf.", o valor do limitante inferior obtido, na coluna "Lim. Sup.", o valor do limitante superior obtido, na coluna "Valor das Sobras", o valor das 
sobras aproveitáveis considerando-se a melhor solução encontrada, na coluna "Placas Usadas", a quantidade de objetos utilizados na solução, na coluna "Iterações MIP”, a quantidade de iterações MIP realizada pelo CPLEX, na coluna "Nós B\&B", a quantidade de nós da árvore Branch \& Bound e na coluna "Tempo", a quantidade de tempo em segundos que levou até a solução ser obtida (ou, caso ela não tenha sido obtida dentro do limite de tempo, exibe o valor deste, que é de 21600 segundos).

Comparando o modelo $\mathcal{M}_{0}^{G} \operatorname{com} \mathcal{M}_{1}^{G}$ e o modelo $\mathcal{M}_{2}^{G}$ com $\mathcal{M}_{3}^{G}$, podemos notar que o uso do dicionário de itens não necessariamente tornou o modelo mais difícil de ser resolvido. Ao compararmos os resultados obtidos para as instâncias 7 e 9 , considerando os modelos $\mathcal{M}_{0}^{G}$ e $\mathcal{M}_{1}^{G}$, por exemplo, notamos que tais instâncias são resolvidas dentro do tempo limite, ao considerarmos o modelo $\mathcal{M}_{1}^{G}$, mas não são resolvidas ao considerarmos o modelo $\mathcal{M}_{0}^{G}$. Comparando os resultados obtidos para a instância 1 , considerando os modelos $\mathcal{M}_{2}^{G}$ e $\mathcal{M}_{3}^{G}$, vemos que o experimento considerando o modelo $\mathcal{M}_{3}^{G}$ foi resolvido mais rapidamente que o experimento considerando o modelo $\mathcal{M}_{2}^{G}$. Note que, no experimento com a instância 5, considerando o modelo $\mathcal{M}_{2}^{G}$, obtivemos um resultado com maior valor de sobras aproveitáveis (menor função objetivo) em comparação com o resultado obtido com o modelo $\mathcal{M}_{3}^{G}$. Isso ocorreu pois, no modelo $\mathcal{M}_{2}^{G}$, para uma sobra ser aproveitável, esta deve ter dimensões horizontais e verticais iguais ou superiores a 3. Considerando o modelo $\mathcal{M}_{3}^{G}$, as sobras aproveitáveis devem ter a dimensão horizontal maior ou igual a 7 e a dimensão vertical maior ou igual a 1. Assim, ao analizarmos a Figura 4.6-(5) e a Figura 4.7-(5), observamos que a sobra hacurada com linhas em 4.6-(5) não é uma sobra aproveitável no contexto do modelo $\mathcal{M}_{3}^{G}$, considerando os itens a serem cortados como elementos do dicionário de itens. Para contornar esse problema, bastaria acrescentar os itens demandados rotacionados de 90 graus ao dicionário de itens. Comparando os modelos $\mathcal{M}_{0}^{G}$ e $\mathcal{M}_{1}^{G}$ com os modelos $\mathcal{M}_{2}^{G}$ e $\mathcal{M}_{3}^{G}$, observamos que o CPLEX demandou mais esforço computacional com os dois últimos em todas as instâncias consideradas. Porém, apesar de mais custosos, estes dois últimos modelos podem propocionar soluções com maiores valores de sobras aproveitáveis. Por exemplo, obtivemos uma solução com maior valor de sobras aproveitáveis, ao resolver a instância 4 com os modelos $\mathcal{M}_{2}^{G}$ e $\mathcal{M}_{3}^{G}$. Podemos afirmar então que considerar sobras genéricas pode de fato ser uma forma de obter soluções com maior valor de sobras aproveitáveis, embora o custo de resolver tais modelos seja maior.

As Figuras 4.2 e 4.3 ilustram as soluções obtidas com o modelo $\mathcal{M}_{0}^{G}$. As Figuras 4.4 e 4.5 ilustram as soluções obtidas com o modelo $\mathcal{M}_{1}^{G}$. A Figura 4.6 ilustra as soluções obtidas com o modelo $\mathcal{M}_{2}^{G}$. A Figura 4.7 ilustra as soluções obtidas com o modelo $\mathcal{M}_{3}^{G}$.

Neste capítulo apresentamos quatro formas distintas de modelar o problema de corte com sobras aproveitáveis. Os experimentos realizados mostraram que com os modelos $\mathcal{M}_{0}^{G} . \mathcal{M}_{1}^{G}, \mathcal{M}_{2}^{G}$ e $\mathcal{M}_{3}^{G}$ fomos capazes de resolver apenas instâncias pequenas do problema. No próximo capítulo introduziremos novas restrições para viabilizar a resolução de algumas das instâncias não resolvidas dentro mesmo do limite de tempo considerado. 


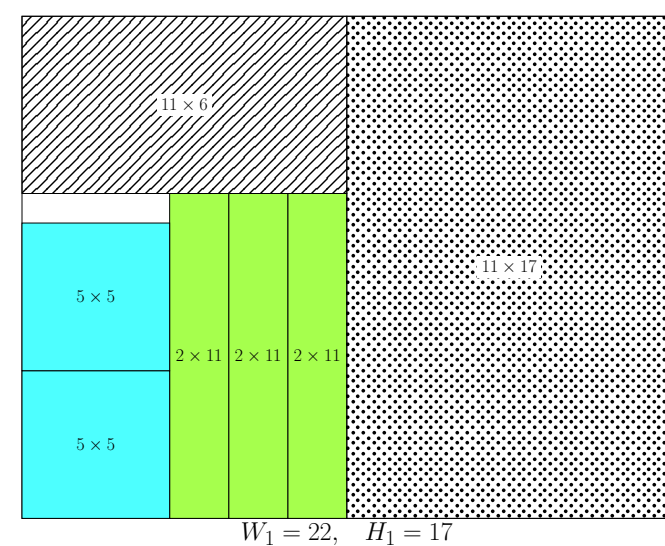

(1)

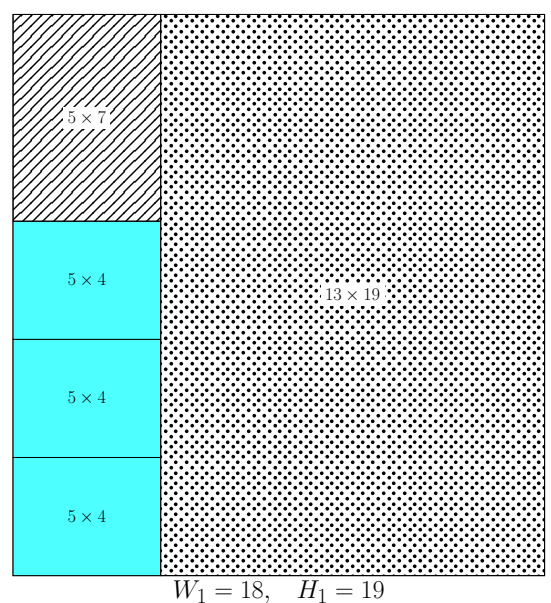

(3)

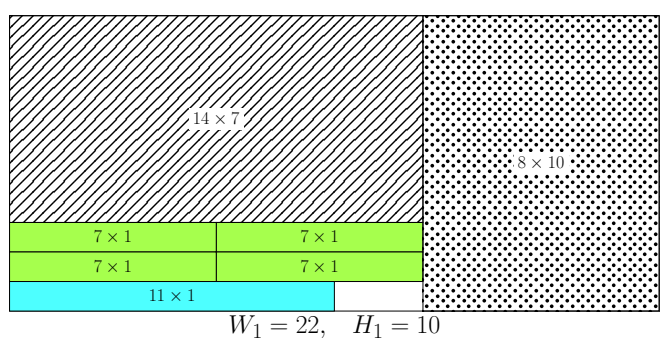

(5)

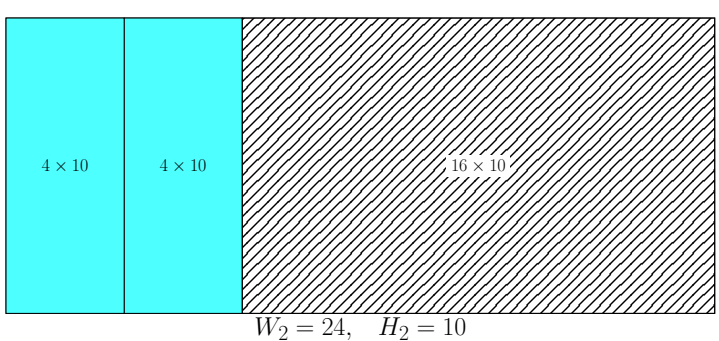

(2)

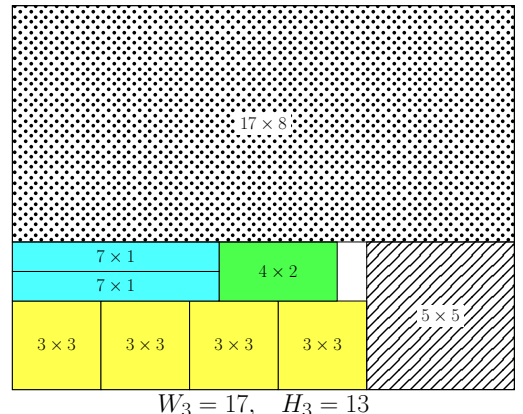

(4)

Figura 4.2: Ilustrações das soluções obtidas pelo modelo $\mathcal{M}_{0}^{G}$ para as instâncias 1, 2, 3, 4 e 5 . 


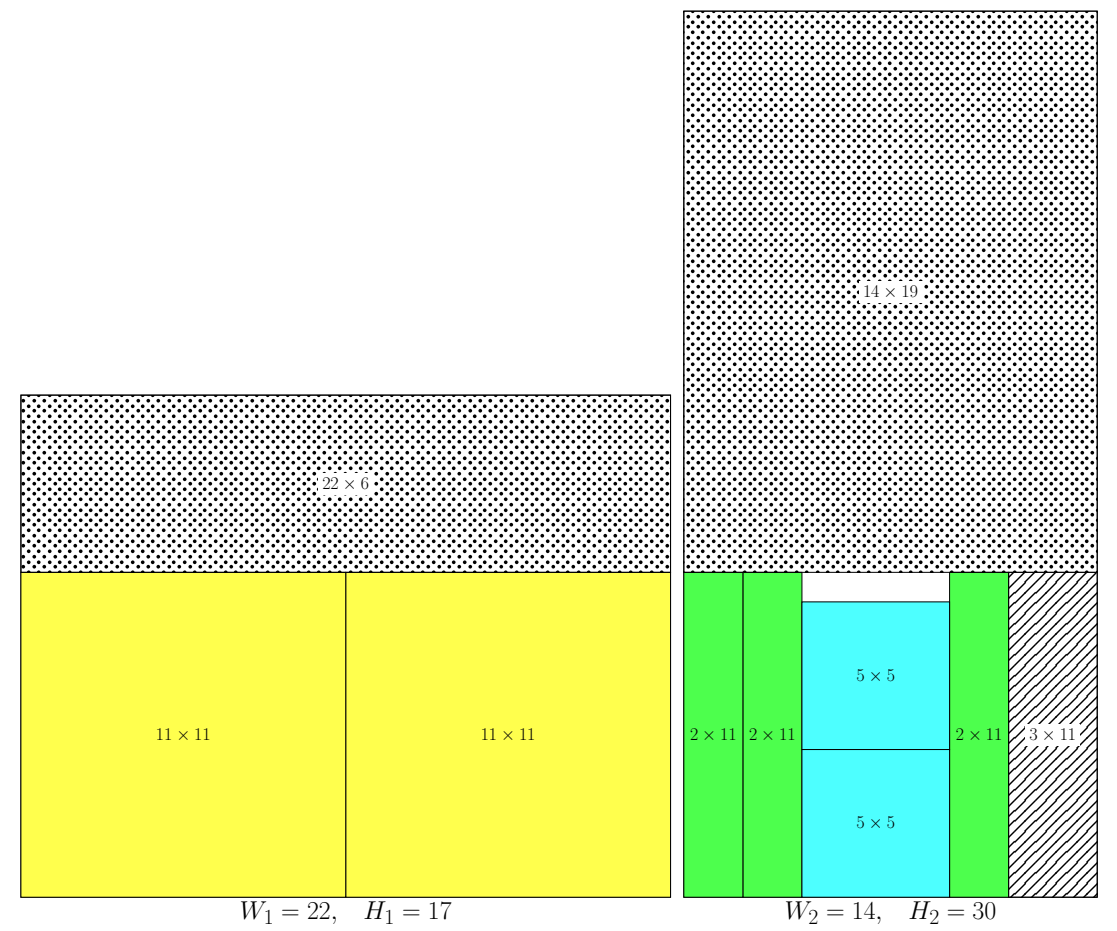

(6)

Figura 4.3: Ilustrações das soluções obtidas pelo modelo $\mathcal{M}_{0}^{G}$ para a instância 6 . 


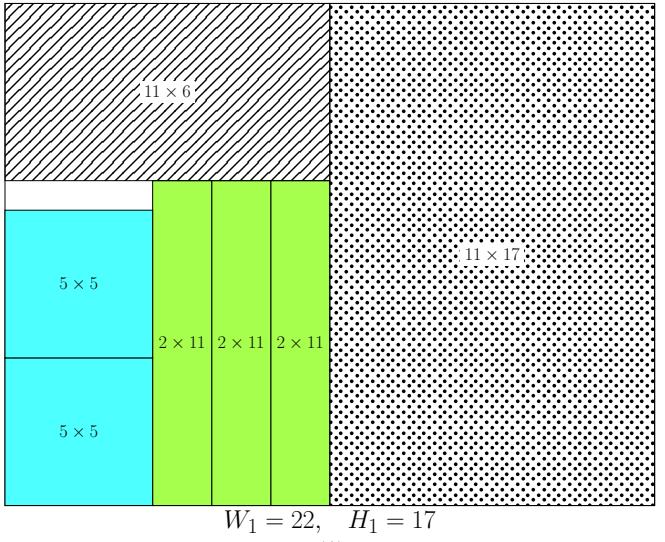

(1)

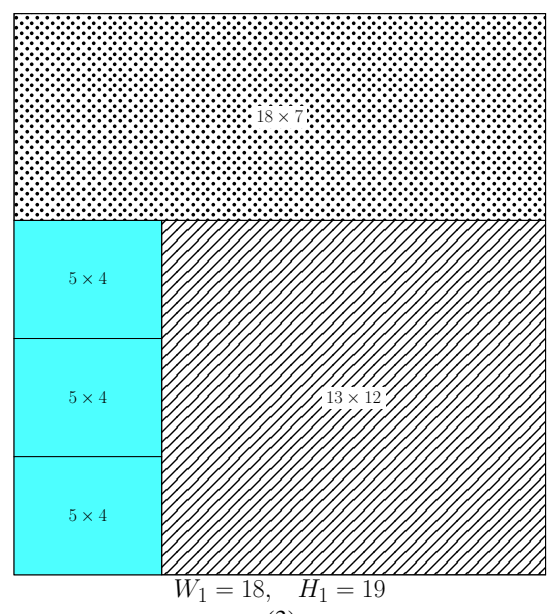

(3)

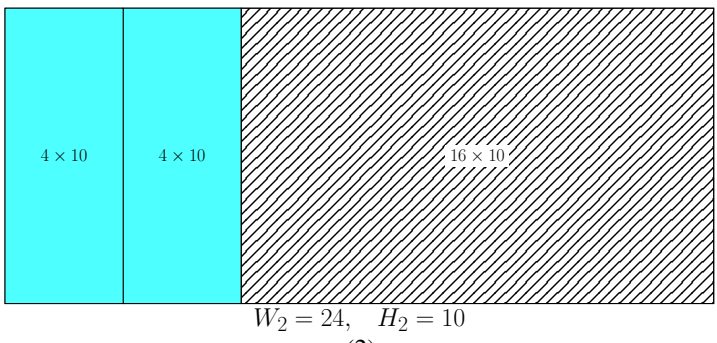

(2)

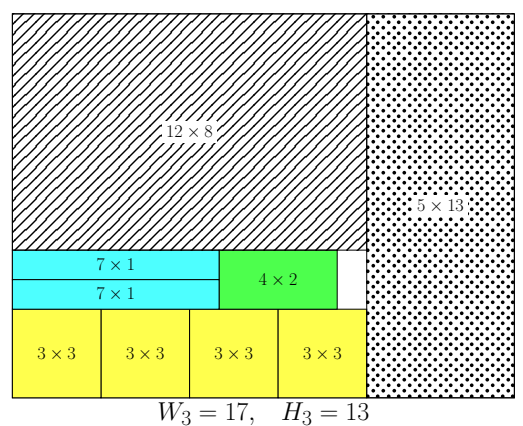

(4)

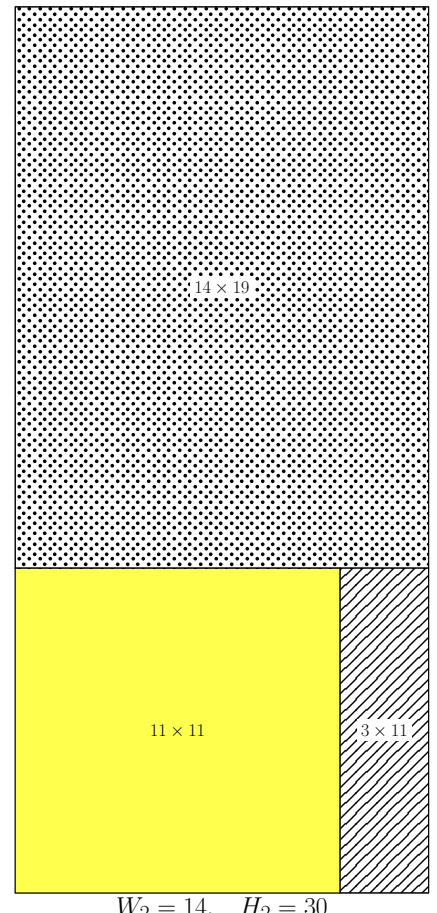

(6)

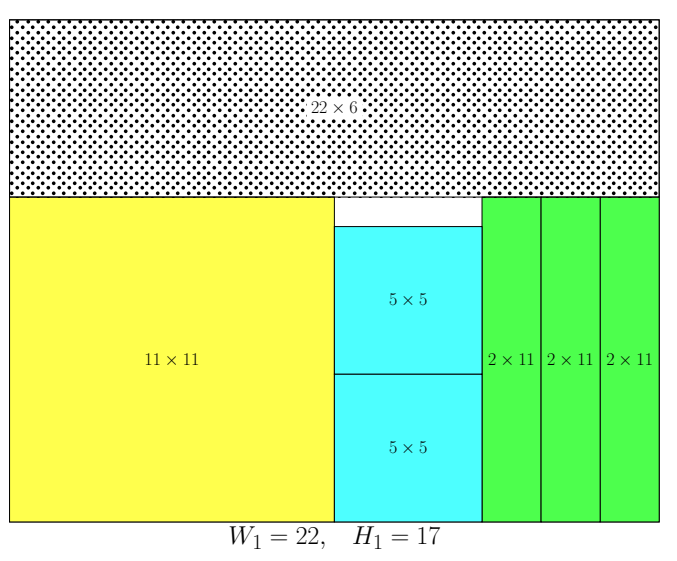

Figura 4.4: Ilustrações das soluções obtidas pelo modelo $\mathcal{M}_{1}^{G}$ para as instâncias 1, 2, 3, 4 e 6 . 


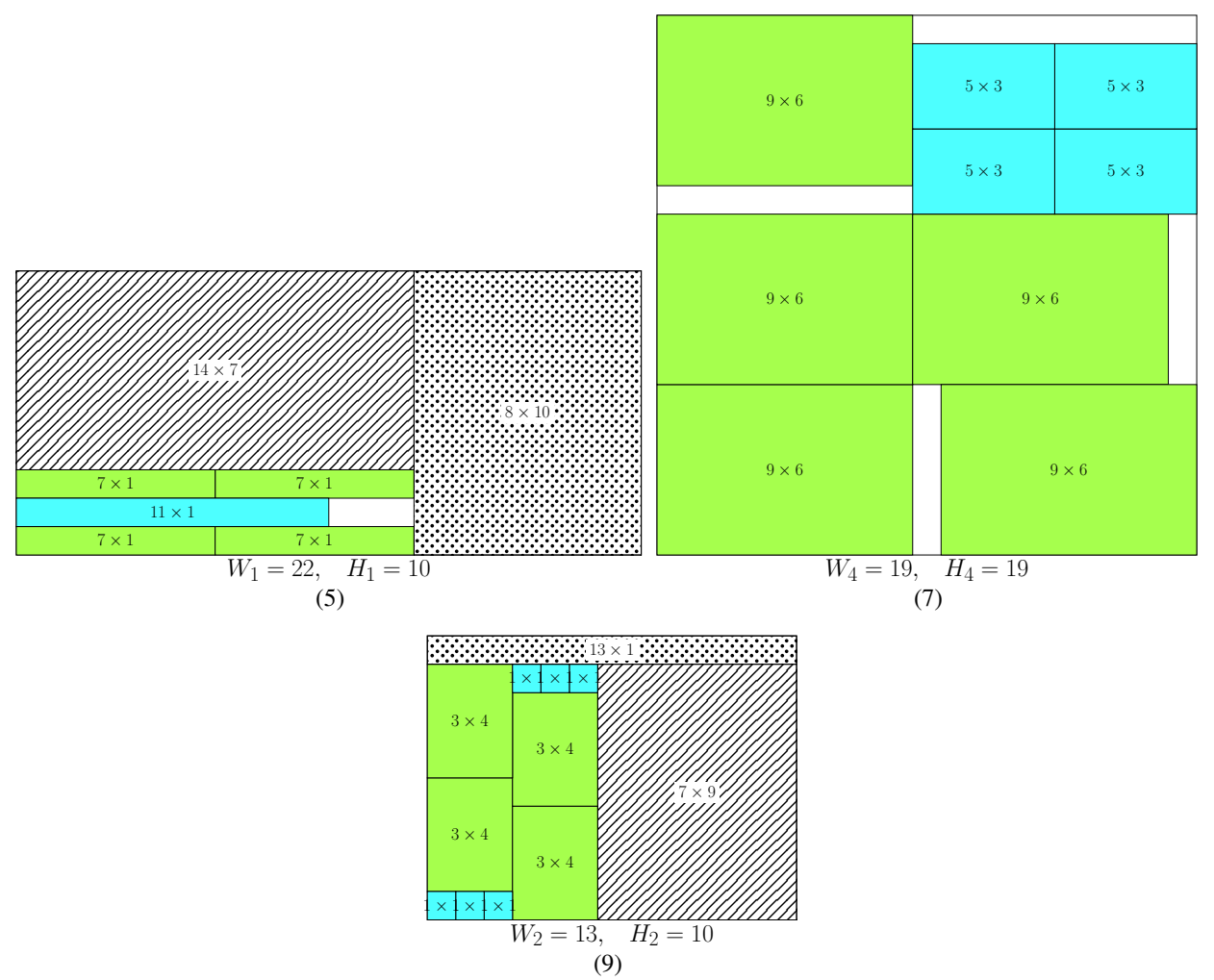

Figura 4.5: Ilustrações das soluções obtidas pelo modelo $\mathcal{M}_{1}^{G}$ para as instâncias 5, 7 e 9 . 


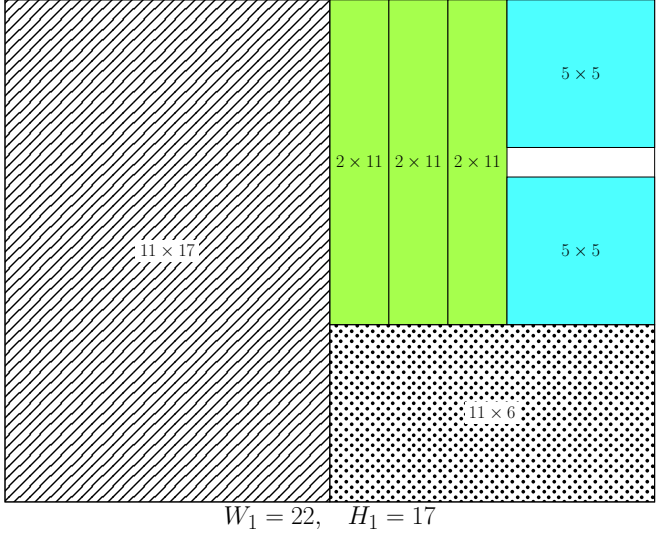

(1)

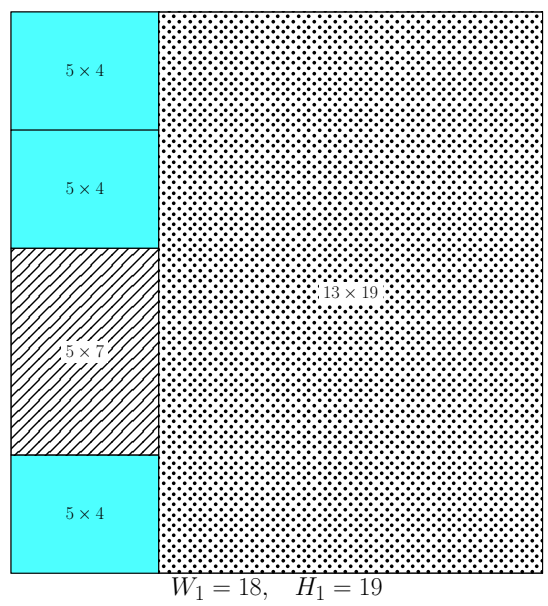

(3)

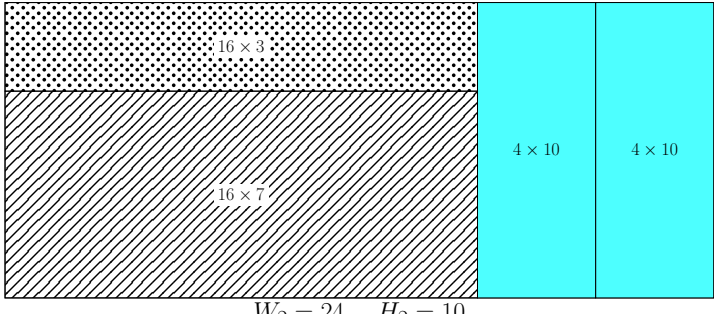

(2)

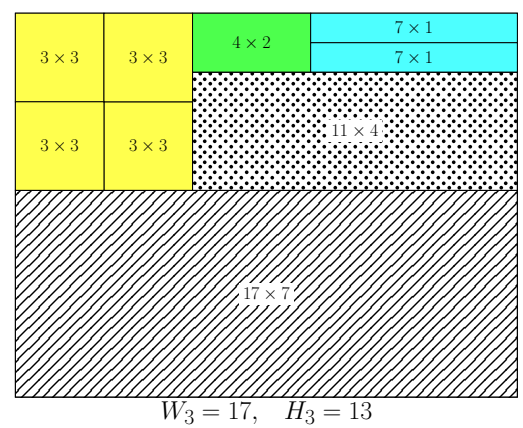

(4)

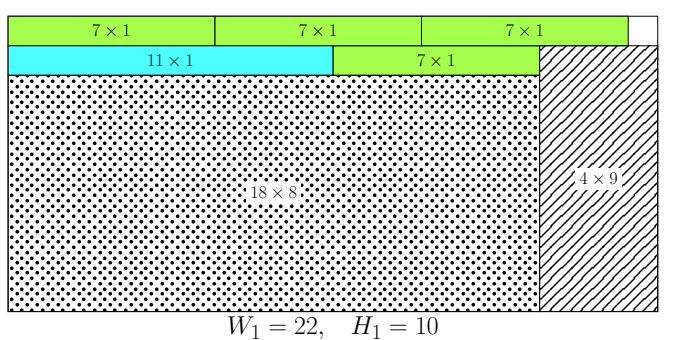

(5)

Figura 4.6: Ilustrações das soluções obtidas pelo modelo $\mathcal{M}_{2}^{G}$ para as instâncias 1, 2, 3, 4 e 5 . 


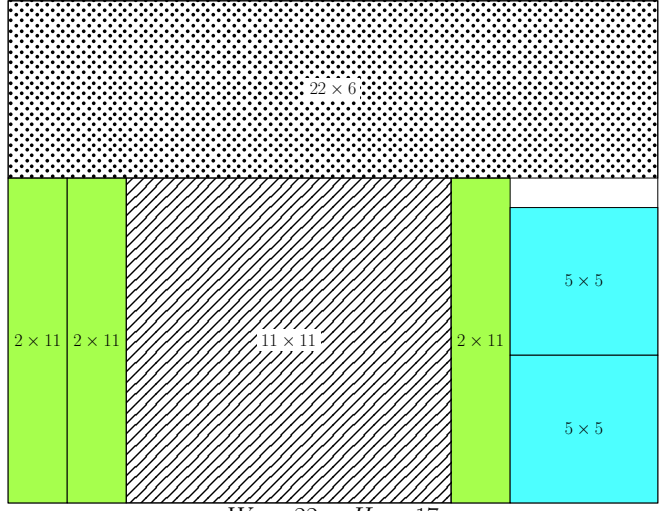

$W_{1}=22, \quad H_{1}=17$

(1)

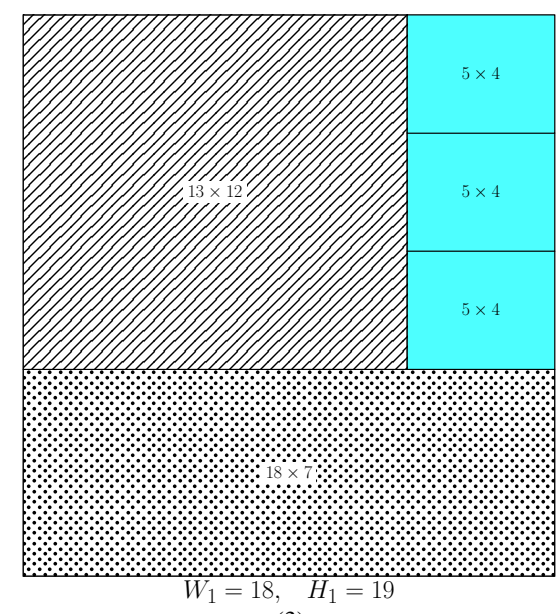

(3)

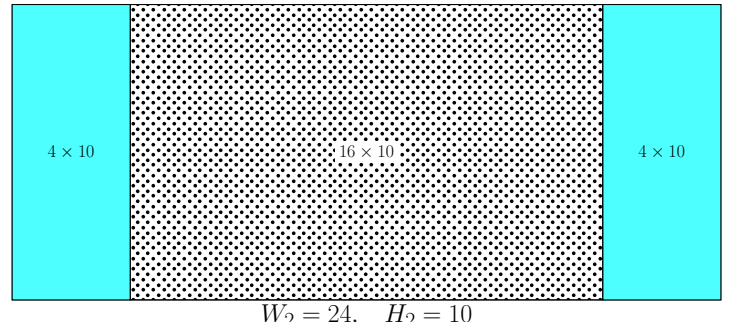

(2)

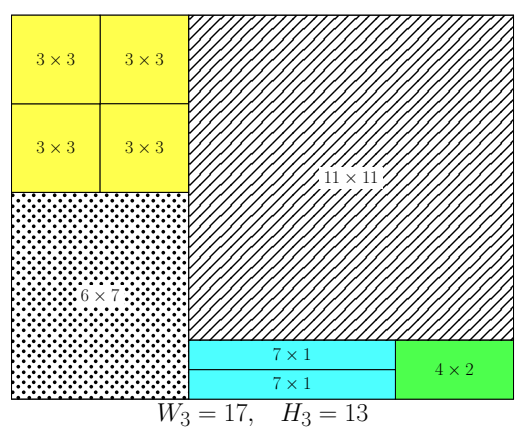

(4)

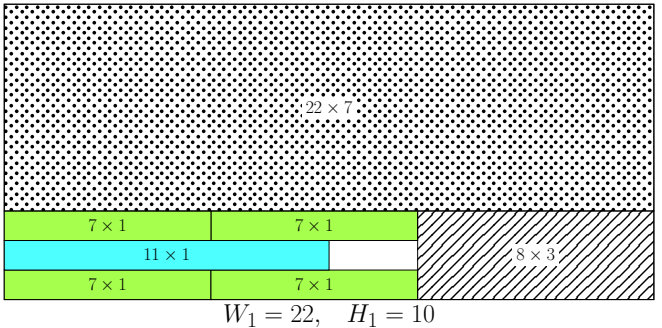

(5)

Figura 4.7: Ilustrações das soluções obtidas pelo modelo $\mathcal{M}_{3}^{G}$ para as instâncias 1, 2, 3, 4 e 5 . 


\section{Capítulo 5}

\section{Melhorias nos modelos para o problema de corte com sobras aproveitáveis}

No Capítulo 4 apresentamos os modelos $\mathcal{M}_{0}^{G}, \mathcal{M}_{1}^{G}, \mathcal{M}_{2}^{G}$ e $\mathcal{M}_{3}^{G}$ para diferentes variações do problema de corte com sobras aproveitáveis. Os modelos $\mathcal{M}_{0}^{G}$ e $\mathcal{M}_{1}^{G}$ correspondem ao problema no qual temos até duas sobras aproveitáveis retangulares do tipo guilhotinado, que devem estar posicionadas nas extremidades superior e direita de cada objeto a ser cortado. Os modelos $\mathcal{M}_{2}^{G}$ e $\mathcal{M}_{3}^{G}$ correspondem ao problema, mais geral, no qual temos até duas sobras aproveitáveis retangulares, sem qualquer restrição sobre seu posicionamento. Neste capítulo apresentamos restrições adicionais para todos esses modelos, com o objetivo de eliminar soluções simétricas e soluções equivalentes. Soluções equivalentes são soluções que possuem o mesmo valor de função objetivo. Duas soluções são ditas simétricas se elas são soluções equivalentes que possuem padrões de corte geometricamente simétricos. A Figura 5.1 ilustra duas soluções equivalentes para uma instância do problema de corte com sobras aproveitáveis do tipo guilhotinado, na qual há um único objeto. A Figura 5.2 ilustra duas soluções simétricas, para outra instância do problema de corte com sobras aproveitáveis guilhotinadas, na qual há apenas um único objeto. Neste caso a simetria está relacionada a permutações das posições dos itens 1 e 3 que são idênticos (mesmo tipo). Como os modelos são resolvidos com um método do tipo branch-and-bound, eliminar soluções equivalentes reduz o espaço de soluções, diminuindo o esforço necessário para enumerar todas as possíveis soluções e tornando o processo de solução mais eficiente.

Apresentamos também uma estratégia alternativa para solucionar os problemas de corte com sobras aproveitáveis através dos modelos propostos. Esta estratégia consiste em resolver inicialmente apenas o problema de minimizar o custo dos objetos e, em seguida, usar o valor do custo ótimo como uma restrição para o problema de maximizar o valor das sobras aproveitáveis.

Finalmente, modelamos uma variação do problema de corte com sobras aproveitáveis, na qual desejamos obter, dentre as soluções que minimizam o custo dos objetos, aquelas que maximizam o custo das sobras e, dentre estas últimas, uma que minimize a quantidade de sobras aproveitáveis. Consideramos que esta variação do problema de corte é uma melhoria pois, de certa forma, eliminamos soluções equivalentes dentro do contexto do problema de corte com sobras aproveitáveis. Em todos os modelos propostos anteriormente podemos ter soluções equivalentes com diferentes quantidades de sobras aproveitáveis, conforme ilustrado na Figura 5.3. Do ponto de vista prático pode ser interessante minimizar a quantidade de sobras aproveitáveis produzidas pois isso poderia, por exemplo, reduzir o custo de estocagem de material. 


\subsection{Restrições de eliminação de soluções equivalentes e simé- tricas}

Em todos os modelos de otimização em dois níveis apresentados para o problema de corte com sobras aproveitáveis, modelamos o valor da sobra como a área da sobra (embora nos MIPs consideremos a área da sobra multiplicada pelo custo do objeto correspondente), caso ela seja aproveitável, e como zero, caso contrário. Apesar disso, não restringimos as dimensões das sobras caso elas não sejam aproveitáveis. Com isso é possível que haja soluções equivalentes com sobras aproveitáveis de tamanhos variados mas valor nulo. A Figura 5.1 ilustra dois exemplos de soluções equivalentes neste contexto. Neste capítulo apresentamos restrições que forçam as dimensões de uma sobra a serem nulas, caso ela não seja aproveitável.

Com relação a eliminação de soluções simétricas, podemos aplicar a ideia apresentada no Capítulo 2, na qual, para um par de itens iguais, pede-se que o de maior índice esteja acima ou à direita do outro, sem se sobreporem. Essencialmente, utilizaremos restrições do tipo (2.11) para modelar a não sobreposição de itens idênticos. Em particular, considerando os modelos $\mathcal{M}_{2}^{G} \mathrm{e}$ $\mathcal{M}_{3}^{G}$, também podemos modelar a não sobreposição das duas possíveis sobras de forma a eliminar soluções simétricas em que as duas sobras aproveitáveis aparecem intercambiadas.

\subsubsection{Eliminação de soluções equivalentes nos modelos $\mathcal{M}_{0}^{G}$ e $\mathcal{M}_{1}^{G}$}

Nos modelos $\mathcal{M}_{0}^{G}$ e $\mathcal{M}_{1}^{G}$, as possíveis sobras aproveitáveis de um objeto $l$ são caracterizadas através das variáveis $\tilde{s}_{l}^{w}$ e $\tilde{h}_{l}^{h}$ e as dimensões horizontal $H_{l}$ e vertical $W_{l}$ do objeto $l$. Mais especificamente, as possíveis sobras aproveitáveis de um objeto $l$ têm dimensões dadas por $W_{l} \times \tilde{s}_{l}^{h}$ e $\tilde{s}_{l}^{w} \times\left(H_{l}-\tilde{s}_{l}^{h}\right)$ ou dadas por $\left(W_{l}-\tilde{s}_{l}^{w}\right) \times \tilde{s}_{l}^{h}$ e $\tilde{s}_{l}^{w} \times H_{l}$. Para forçar as dimensões das sobras a serem nulas, caso elas não sejam aproveitáveis, e lembrando que $M_{l}^{W}$ e $M_{l}^{H}$ são constantes suficientemente grandes e que $z_{l}, \bar{z}_{l} \in\{0,1\}$, basta adicionar as seguintes restrições ao modelo $\mathcal{M}_{0}^{G}$ :

$$
\begin{aligned}
& \tilde{s}_{l}^{h} \leq M_{l}^{H}\left(1-z_{l}\right), \\
& \tilde{s}_{l}^{w} \leq M_{l}^{W}\left(1-\bar{z}_{l}\right),
\end{aligned}
$$

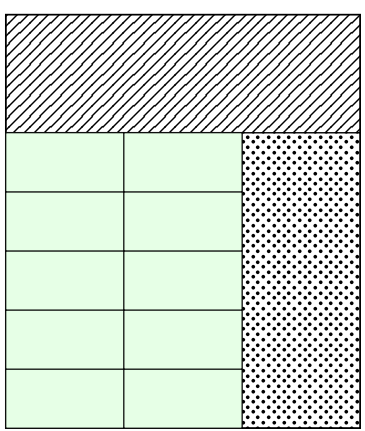

(a)

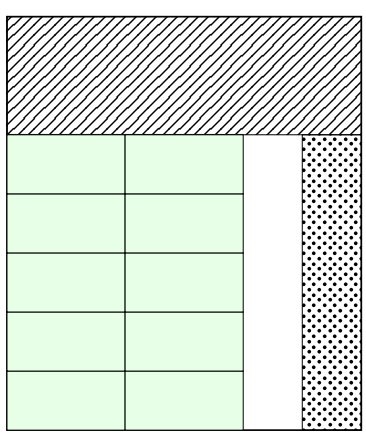

(b)

Figura 5.1: (a) e (b) representam duas soluções equivalentes de uma instância do modelo $M_{0}^{G}$. Essa instância é caracterizada por 10 itens de $2 \times 1,1$ objeto de $6 \times 7$ e dimensões mínimas $w_{\min }=h_{\min }=3$ para caracterizar as sobras aproveitáveis. As sobras indicadas com hachura de pontos têm tamanhos diferentes nas duas soluções, mas, por conta de suas dimensões, não são aproveitáveis e têm valor nulo. 
para $l=1, \ldots, p$, e as seguintes restrições ao modelo $\mathcal{M}_{1}^{G}$ :

$$
\begin{aligned}
& \tilde{s}_{l}^{h} \leq M_{l}^{H} \sum_{i=1}^{n}\left(1-\tau_{i l}\right) \\
& \tilde{s}_{l}^{w} \leq M_{l}^{W} \sum_{i=1}^{n}\left(1-\bar{\tau}_{i l}\right)
\end{aligned}
$$

para $l=1, \ldots, p$, lembrando que $\left.\tau_{i l}, \bar{\tau}_{i l}\right) \in\{0,1\}$.

\subsubsection{Eliminação de soluções equivalentes nos Modelos $\mathcal{M}_{2}^{G}$ e $\mathcal{M}_{3}^{G}$}

Nos modelos $\mathcal{M}_{2}^{G}$ e $\mathcal{M}_{3}^{G}$, as possíveis sobras aproveitáveis de um objeto $l$ são caracterizadas pelas variáveis $s_{l}^{w}, s_{l}^{h}, t_{l}^{w}$ e $t_{l}^{h}$, mais especificamente temos a sobra de dimensões $s_{l}^{w} \times s_{l}^{h}$ e a sobra de dimensões $t_{l}^{w} \times t_{l}^{h}$. Considerando o modelo $\mathcal{M}_{2}^{G}$, para forçar as dimensões das sobras a serem nulas, caso estas não sejam aproveitáveis, basta adicionar as seguintes restrições:

$$
\begin{aligned}
s_{l}^{w} & \leq M_{l}^{W}\left(1-z_{l}\right), \\
s_{l}^{h} & \leq M_{l}^{H}\left(1-z_{l}\right), \\
t_{l}^{w} & \leq M_{l}^{W}\left(1-\bar{z}_{l}\right), \\
t_{l}^{h} & \leq M_{l}^{H}\left(1-\bar{z}_{l}\right),
\end{aligned}
$$
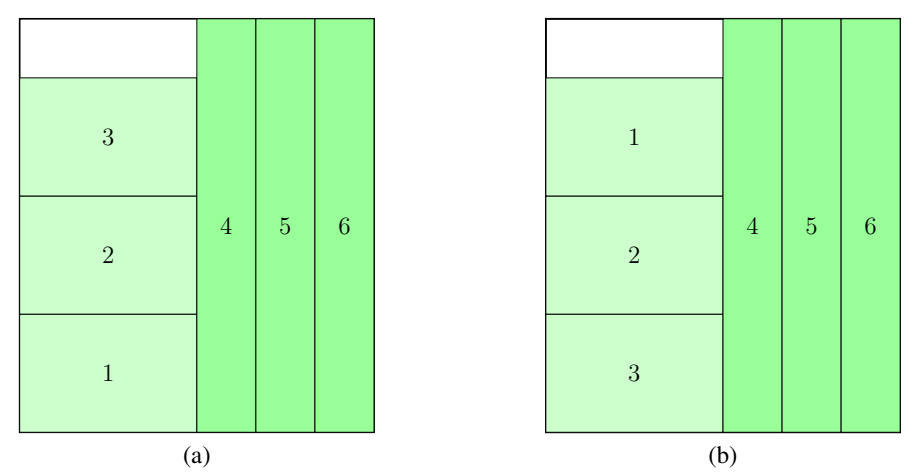

Figura 5.2: (a) e (b) representam duas soluções simétricas de uma instância do modelo $M_{0}^{G}$. Essa instância é caracterizada por 3 itens de $3 \times 2$, 3 itens de $1 \times 7,1$ objeto de $6 \times 7$ e dimensões mínimas $w_{\min }=h_{\min }=3$ para caracterizar as sobras aproveitáveis. As soluções diferem apenas por uma troca de posição de dois itens do mesmo tipo, os itens 1 e 3. 


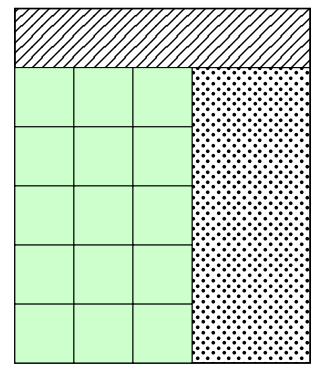

(a)

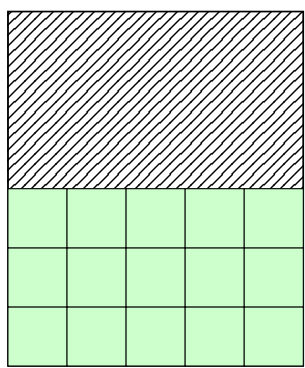

(b)

Figura 5.3: $(a)$ e (b) representam duas soluções equivalentes de uma instância do modelo $M_{0}^{G}$. Essa instância é caracterizada por 15 itens de $1 \times 1,1$ objeto de $5 \times 6$ e dimensões mínimas $w_{\min }=h_{\min }=1$ para caracterizar as sobras aproveitáveis. Ambas as soluções são equivalentes porque possuem o mesmo custo dos objetos utilizados e mesmo valor nas sobras aproveitáveis. Porém, do ponto de vista prático, a solução ilustrada em (b) é preferível por ter uma única sobra aproveitável.

para $l=1, \ldots, p$. Considerando o modelo $\mathcal{M}_{3}^{G}$ devemos acrescentar as seguintes restrições:

$$
\begin{aligned}
& s_{l}^{w} \leq M_{l}^{W} \sum_{i=1}^{n}\left(1-\tau_{i l}\right), \\
& s_{l}^{h} \leq M_{l}^{H} \sum_{i=1}^{n}\left(1-\tau_{i l}\right), \\
& t_{l}^{w} \leq M_{l}^{W} \sum_{i=1}^{n}\left(1-\bar{\tau}_{i l}\right), \\
& t_{l}^{h} \leq M_{l}^{H} \sum_{i=1}^{n}\left(1-\bar{\tau}_{i l}\right),
\end{aligned}
$$

para $l=1, \ldots, p$.

\subsubsection{Restrições de eliminação de simetrias do tipo "acima e à direita" para itens do mesmo tipo}

Neste caso, substituiremos as restrições de não sobreposição entre itens do mesmo tipo nos modelos $\mathcal{M}_{0}^{G}, \mathcal{M}_{1}^{G}, \mathcal{M}_{2}^{G}$ e $\mathcal{M}_{3}^{G}$ por restrições mais simples na forma de (2.12). Assim, caso dois itens $i$ e $k$ tenham as mesmas dimensões horizontal e vertical, ou seja, sejam do mesmo tipo, 
substituiremos:

$$
\begin{aligned}
\frac{w_{i}+w_{k}}{2}-\left(x_{i}-x_{k}\right) \leq & M_{l}^{W} \eta_{i k}+M_{l}^{W} \bar{\eta}_{i k}+ \\
& M_{l}^{W}\left(1-\bar{u}_{i l}\right)+M_{l}^{W}\left(1-\bar{u}_{k l}\right), \\
\frac{w_{i}+w_{k}}{2}+\left(x_{k}-x_{i}\right) \leq & M_{l}^{W}\left(1-\eta_{i k}\right)+M_{l}^{W} \bar{\eta}_{i k}+ \\
& M_{l}^{W}\left(1-\bar{u}_{i l}\right)+M_{l}^{W}\left(1-\bar{u}_{k l}\right), \\
\frac{h_{i}+h_{k}}{2}-\left(y_{i}-y_{k}\right) \leq & M_{l}^{H} \eta_{i k}+M_{l}^{H}\left(1-\bar{\eta}_{i k}\right)+ \\
& M_{l}^{H}\left(1-\bar{u}_{i l}\right)+M_{l}^{H}\left(1-\bar{u}_{k l}\right), \\
\frac{h_{i}+h_{k}}{2}+\left(y_{k}-y_{i}\right) \leq & M_{l}^{H}\left(1-\eta_{i k}\right)+M_{l}^{H}\left(1-\bar{\eta}_{i k}\right), \\
& M_{l}^{H}\left(1-\bar{u}_{i l}\right)+M_{l}^{H}\left(1-\bar{u}_{k l}\right),
\end{aligned}
$$

por:

$$
\begin{aligned}
& \frac{w_{i}+w_{k}}{2}+\left(x_{k}-x_{i}\right) \leq M_{l}^{W} \eta_{i k}+M_{l}^{W}\left(1-\bar{u}_{i l}\right)+M_{l}^{W}\left(1-\bar{u}_{k l}\right) \\
& \frac{h_{i}+h_{k}}{2}+\left(y_{k}-y_{i}\right) \leq M_{l}^{H}\left(1-\eta_{i k}\right)+M_{l}^{H}\left(1-\bar{u}_{i l}\right)+M_{l}^{H}\left(1-\bar{u}_{k l}\right),
\end{aligned}
$$

para $l=1, \ldots, p$.

\subsubsection{Restrições de eliminação de simetrias específicas para os Modelos $\mathcal{M}_{2}^{G}$ e $\mathcal{M}_{3}^{G}$}

Nos modelos $\mathcal{M}_{2}^{G}$ e $\mathcal{M}_{3}^{G}$ as sobras aproveitáveis são modeladas como itens adicionais, com dimensões variáveis. Por conta disso, é possível trocar as duas sobras de lugar intercambiando suas dimensões e posições. Assim, qualquer solução para esses modelos possui uma solução simétrica e, para obtê-la, basta trocar $s_{l}^{w} \operatorname{com} t_{l}^{w}, s_{l}^{h} \operatorname{com} t_{l}^{h}, x_{l}^{s} \operatorname{com} x_{l}^{t}$ e $y_{l}^{s} \operatorname{com} y_{l}^{t}$. Portanto, podemos considerar a mesma ideia utilizada em (2.11) e pedir que a sobra aproveitável definida pelas dimensões $t_{l}^{w}$ e $t_{l}^{h}$ esteja sempre acima ou à direita da sobra definida por $s_{l}^{w}$ e $s_{l}^{h}$. Ou seja, basta trocar as restrições:

$$
\begin{aligned}
& \frac{\left(s_{l}^{w}+t_{l}^{w}\right)}{2}-\left(x_{l}^{t}-x_{l}^{s}\right) \leq M^{A}\left(1-u_{l}\right)+M^{A} r_{l}+M^{A} \bar{r}_{l}, \\
& \frac{\left(s_{l}^{w}+t_{l}^{w}\right)}{2}+\left(x_{l}^{t}-x_{l}^{s}\right) \leq M^{A}\left(1-u_{l}\right)+M^{A}\left(1-r_{l}\right)+M^{A} \bar{r}_{l}, \\
& \frac{\left(s_{l}^{h}+t_{l}^{h}\right)}{2}-\left(y_{l}^{t}-y_{l}^{s}\right) \leq M^{A}\left(1-u_{l}\right)+M_{l}^{A}\left(1-r_{l}\right)+M^{A} \bar{r}_{l}, \\
& \frac{\left(s_{l}^{h}+t_{l}^{h}\right)}{2}+\left(y_{l}^{t}-y_{l}^{s}\right) \leq M^{A}\left(1-u_{l}\right)+M_{l}^{A}\left(1-r_{l}\right)+M^{A}\left(1-\bar{r}_{l}\right),
\end{aligned}
$$


por:

$$
\begin{aligned}
& \frac{\left(s_{l}^{w}+t_{l}^{w}\right)}{2}-\left(x_{l}^{t}-x_{l}^{s}\right) \leq M^{A}\left(1-u_{l}\right)+M^{A} r_{l}, \\
& \frac{\left(s_{l}^{h}+t_{l}^{h}\right)}{2}-\left(y_{l}^{t}-y_{l}^{s}\right) \leq M^{A}\left(1-u_{l}\right)+\left(1-M^{A} r_{l}\right),
\end{aligned}
$$

para $l=1, \ldots, p$.

\subsubsection{Experimentos}

Nesta seção apresentamos alguns resultados obtidos a partir de experimentos numéricos com os modelos $\mathcal{M}_{0}^{G}, \mathcal{M}_{1}^{G}, \mathcal{M}_{2}^{G}$ e $\mathcal{M}_{3}^{G}$, acrescidos das restrições de eliminação de soluções equivalentes e simétricas. As instâncias utilizadas nos experimentos foram as mesmas descritas na Tabela 4.3.

Os modelos foram implementados utilizando o CPLEX 12.1 e a Concert Techonology 2.9. Os programas foram compilados utilizando o compilador G++ 4.4.3 da GCC (GNU Compiller Collection) e os experimentos realizados em uma máquina com $8 \mathrm{~GB}$ de memória RAM, dois processadores de $2.6 \mathrm{GHz}$ Intel Xeon, com 6 núcleos cada um e tecnologia Hyper Threading. Com relação às opções de configuração do CPLEX, bem como, constantes e demais valores relativos à caracterização das sobras nos modelos, utilizamos exatamente os mesmo valores descritos na seção 4.4 .

A Tabela 5.1 sumariza os experimentos numéricos com os modelos $\mathcal{M}_{0}^{G}, \mathcal{M}_{1}^{G}, \mathcal{M}_{2}^{G}$ e $\mathcal{M}_{3}^{G}$, acrescidos das restrições de eliminação de soluções equivalentes e simétricas. Os modelos modificados são chamados respectivamente de $\mathcal{S}_{0}^{G}, \mathcal{S}_{1}^{G}, \mathcal{S}_{2}^{G}$ e $\mathcal{S}_{3}^{G}$. Para cada instância informamos na coluna "Lim. Inf." o valor do limitante inferior obtido, na coluna "Lim. Sup." o valor do limitante superior obtido, na coluna "V. Sobras" o valor das sobras aproveitáveis considerando-se a melhor solução encontrada, na coluna "Placas Usadas" a quantidade de objetos utilizados na solução, na coluna "Iterações MIP" a quantidade de iterações MIP realizada pelo CPLEX, na coluna "Nós B\&B" a quantidade de nós da árvore Branch \& Bound e na coluna "Tempo" a quantidade de tempo em segundos que levou até a solução ser obtida (ou, caso ela não tenha sido obtida dentro do limite de tempo, exibe o valor deste, que é de 21600 segundos). Comparando a Tabela $5.1 \mathrm{com}$ a Tabela 4.4, observamos uma melhora no desempenho de resolução de todas as instâncias, considerando todos os modelos. Mais especificamente, considerando os experimentos envolvendo os modelos $\mathcal{S}_{0}^{G}$ e $\mathcal{S}_{1}^{G}$, fomos capazes de resolver todas as instâncias dentro do tempo limite, utilizando as restrições adicionais. As Figuras 5.4-5.7 ilustram as soluções obtidas. Observe que em 5.5-(9) não temos a sobra de dimensão $13 \times 1$ que podemos notar em 5.7-(9), por causa das diferenças com relação à definição de sobra aproveitável nos modelos $\mathcal{S}_{0}^{G}$ e $\mathcal{S}_{1}^{G}$. Essa mesma diferença pode ser percebida ao observarmos o valor das sobras aproveitáveis obtido para a instância 9 considerando tais modelos na Tabela 4.4. Com relação aos experimentos envolvendo os modelos $\mathcal{S}_{2}^{G}$ e $\mathcal{S}_{3}^{G}$, também obtivemos ganhos de eficiência. Conseguimos reduzir o esforço computacional em mais de $90 \%$ ao resolver a instância 4 , por exemplo. Com o modelo $\mathcal{S}_{2}^{G}$ conseguimos resolver adicionalmente as instâncias 8 e 10 e com o modelo $\mathcal{S}_{3}^{G}$ conseguimos resolver adicionalmente as instâncias 7, 8 e 9. As ilustrações dos resultados com essas instâncias podem ser observados nas Figuras 5.9-5.11. 


\begin{tabular}{|c|c|c|c|c|c|c|c|}
\hline \multicolumn{8}{|c|}{ Modelo $\mathcal{S}_{0}^{G}$} \\
\hline \multirow[t]{2}{*}{ \# Instância } & \multicolumn{4}{|c|}{ Dados das soluções } & \multicolumn{3}{|c|}{ Medidas de desempenho } \\
\hline & Lim. Inf. & Lim. Sup. & V. Sobras & Placas Usadas & Iterações MIP & Nós $B \& B$ & Tempo (s) \\
\hline 1 & 373,700824596239 & 373,700824596239 & 94622 & 1 & 280 & 67 & 0,12 \\
\hline 2 & 239,872276308918 & 239,872276308918 & 38400 & 1 & 70 & 1 & 0,06 \\
\hline 3 & 341,782856165062 & 341,782856165062 & 96444 & 1 & 186 & 13 & 0,05 \\
\hline 4 & 220,826167037155 & 220,826167037155 & 35581 & 1 & 7800 & 3028 & 0,46 \\
\hline 5 & 219,768973003587 & 219,768973003587 & 39160 & 1 & 577 & 176 & 0,15 \\
\hline 6 & 793,446850219429 & 793,446850219429 & 174948 & 2 & 1309739 & 566687 & 29,23 \\
\hline 7 & 361,000000000000 & 361,000000000000 & 0 & 1 & 5393 & 831 & 0,41 \\
\hline 8 & 175,825697498245 & 175,825697498245 & 23584 & 1 & 396937 & 110550 & 7,81 \\
\hline 9 & 129,937883959044 & 129,937883959044 & 9100 & 1 & 49967 & 16943 & 1,49 \\
\hline 10 & 307,851923076923 & 307,851923076923 & 37268 & 1 & 191591 & 76488 & 5,01 \\
\hline \multicolumn{8}{|c|}{ Modelo $\mathcal{S}_{1}^{G}$} \\
\hline \multirow[t]{2}{*}{ \# Instância } & \multicolumn{4}{|c|}{ Dados das soluções } & \multicolumn{3}{|c|}{ Medidas de desempenho } \\
\hline & Lim. Inf. & Lim. Sup. & V. Sobras & Placas Usadas & Iterações MIP & Nós B\&B & Tempo (s) \\
\hline 1 & 373,700824596224 & 373,700824596224 & 94622 & 1 & 370 & 84 & 0,15 \\
\hline 2 & 239,872276308918 & 239,872276308918 & 38400 & 1 & 75 & 0 & 0,08 \\
\hline 3 & 341,782856165062 & 341,782856165062 & 96444 & 1 & 138 & 8 & 0,10 \\
\hline 4 & 220,826167037155 & 220,826167037155 & 35581 & 1 & 9811 & 3650 & 0,57 \\
\hline 5 & 219,768973003587 & 219,768973003587 & 39160 & 1 & 903 & 246 & 0,19 \\
\hline 6 & 793,446850219429 & 793,446850219429 & 174948 & 2 & 5744500 & 2751376 & 198,39 \\
\hline 7 & 361,000000000000 & 361,000000000000 & 0 & 1 & 24816 & 3412 & 0,73 \\
\hline 8 & 175,825697498245 & 175,825697498245 & 23584 & 1 & 212959 & 77446 & 6,67 \\
\hline 9 & 129,932559726962 & 129,932559726962 & 9880 & 1 & 30808 & 8367 & 1,32 \\
\hline 10 & 307,851923076923 & 307,851923076923 & 37268 & 1 & 313915 & 148651 & 9,99 \\
\hline \multicolumn{8}{|c|}{ Modelo $\mathcal{S}_{2}^{G}$} \\
\hline \multirow[t]{2}{*}{ \# Instância } & \multicolumn{4}{|c|}{ Dados das soluções } & \multicolumn{3}{|c|}{ Medidas de desempenho } \\
\hline & Lim. Inf. & Lim. Sup. & V. Sobras & Placas Usadas & Iterações MIP & Nós B\&B & Tempo (s) \\
\hline 1 & 373,700824596226 & 373,700824596226 & 94622 & 1 & 68684 & 18364 & 2,07 \\
\hline 2 & 239,872276308918 & 239,872276308918 & 38400 & 1 & 675 & 151 & 0,14 \\
\hline 3 & 341,782856165051 & 341,782856165051 & 96444 & 1 & 4711 & 1757 & 0,31 \\
\hline 4 & 220,824007621455 & 220,824007621455 & 36023 & 1 & 7634174 & 1488647 & 140,22 \\
\hline 5 & 219,766377194615 & 219,766377194615 & 39600 & 1 & 260659 & 44852 & 5,39 \\
\hline 6 & 792,904210246742 & 793,440937662042 & - & - & - & - & $\geq 21601,00$ \\
\hline 7 & 360,984798951039 & 361,000000000000 & - & - & - & - & $\geq 21600,00$ \\
\hline 8 & 175,825682716804 & 175,825697498241 & 23584 & 1 & 586580819 & 74875579 & 7181,52 \\
\hline 9 & 129,930784982932 & 129,932559704947 & - & - & - & - & $\geq 21600,00$ \\
\hline 10 & 307,835988925723 & 307,836013985997 & 41272 & 1 & 277359144 & 56782913 & 4473,46 \\
\hline \multicolumn{8}{|c|}{ Modelo $\mathcal{S}_{3}^{G}$} \\
\hline \multirow[t]{2}{*}{ \# Instância } & \multicolumn{4}{|c|}{ Dados das soluções } & \multicolumn{3}{|c|}{ Medidas de desempenho } \\
\hline & Lim. Inf. & Lim. Sup. & V. Sobras & Placas Usadas & Iterações MIP & Nós B\&B & Tempo (s) \\
\hline 1 & 373,700824596239 & 373,700824596239 & 94622 & 1 & 92628 & 27165 & 3,10 \\
\hline 2 & 239,872276308918 & 239,872276308918 & 38400 & 1 & 731 & 176 & 0,16 \\
\hline 3 & 341,782856165044 & 341,782856165044 & 96444 & 1 & 8777 & 2191 & 0,39 \\
\hline 4 & 220,824007621449 & 220,824007621449 & 36023 & 1 & 13645255 & 2747961 & 263,40 \\
\hline 5 & 219,768973003569 & 219,768973003569 & 39160 & 1 & 1151266 & 161238 & 14,21 \\
\hline 6 & 793,143419039067 & 793,440937662042 & - & - & - & - & $\geq 21600,00$ \\
\hline 7 & 361,000000000000 & 361,000000000000 & 0 & 1 & 421168759 & 142948635 & 18558,57 \\
\hline 8 & 175,825697328555 & 175,825697419926 & 23584 & 1 & 197526655 & 31019382 & 2925,20 \\
\hline 9 & 129,932559726962 & 129,932559726962 & 9880 & 1 & 122515265 & 21669118 & 2025,17 \\
\hline 10 & 307,831118881092 & 307,833566433560 & - & - & - & - & $\geq 21600,00$ \\
\hline
\end{tabular}

Tabela 5.1: Resultados dos experimentos com os modelos $\mathcal{S}_{0}^{G}, \mathcal{S}_{1}^{G}, \mathcal{S}_{2}^{G}$ e $\mathcal{S}_{3}^{G}$. 


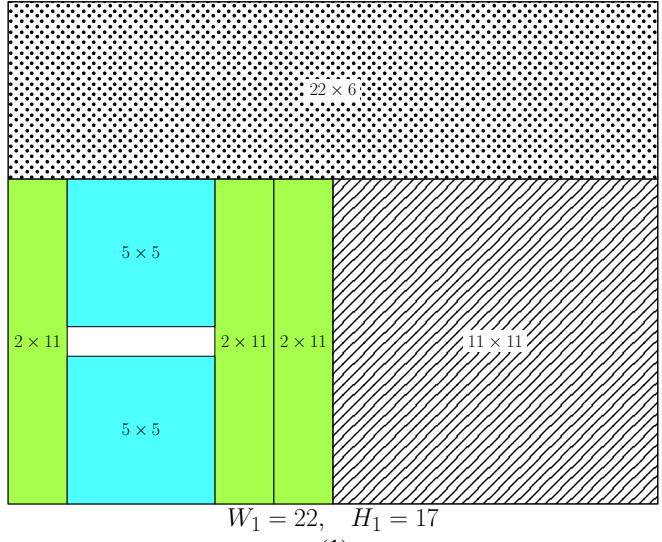

(1)

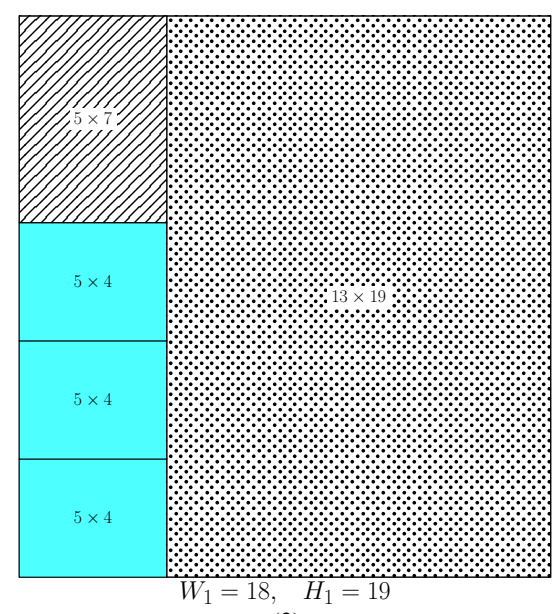

(3)

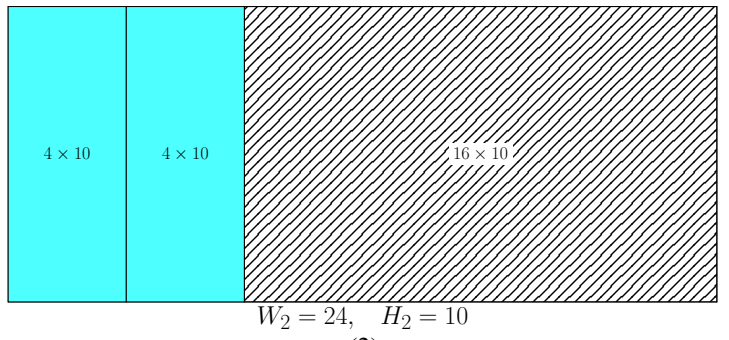

(2)

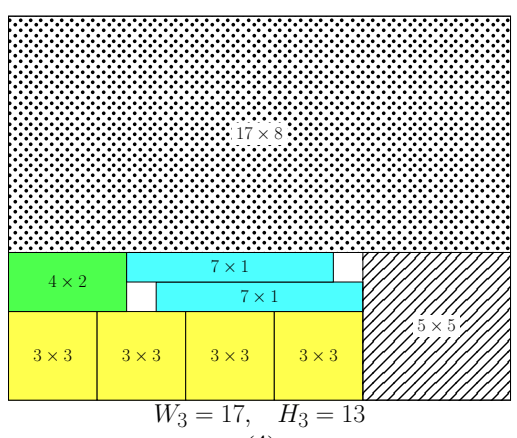

(4)

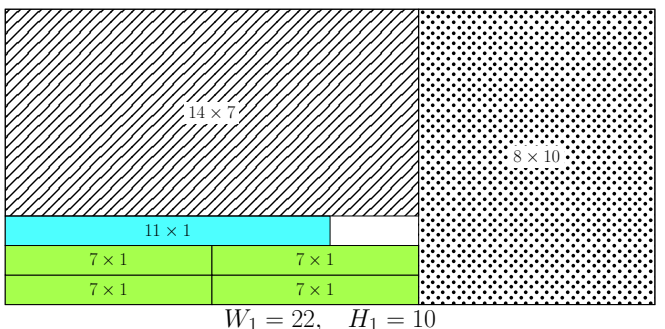

(5)

Figura 5.4: Ilustrações das soluções obtidas pelo modelo $\mathcal{S}_{0}^{G}$ para as instâncias 1, 2, 3, 4 e 5 . 

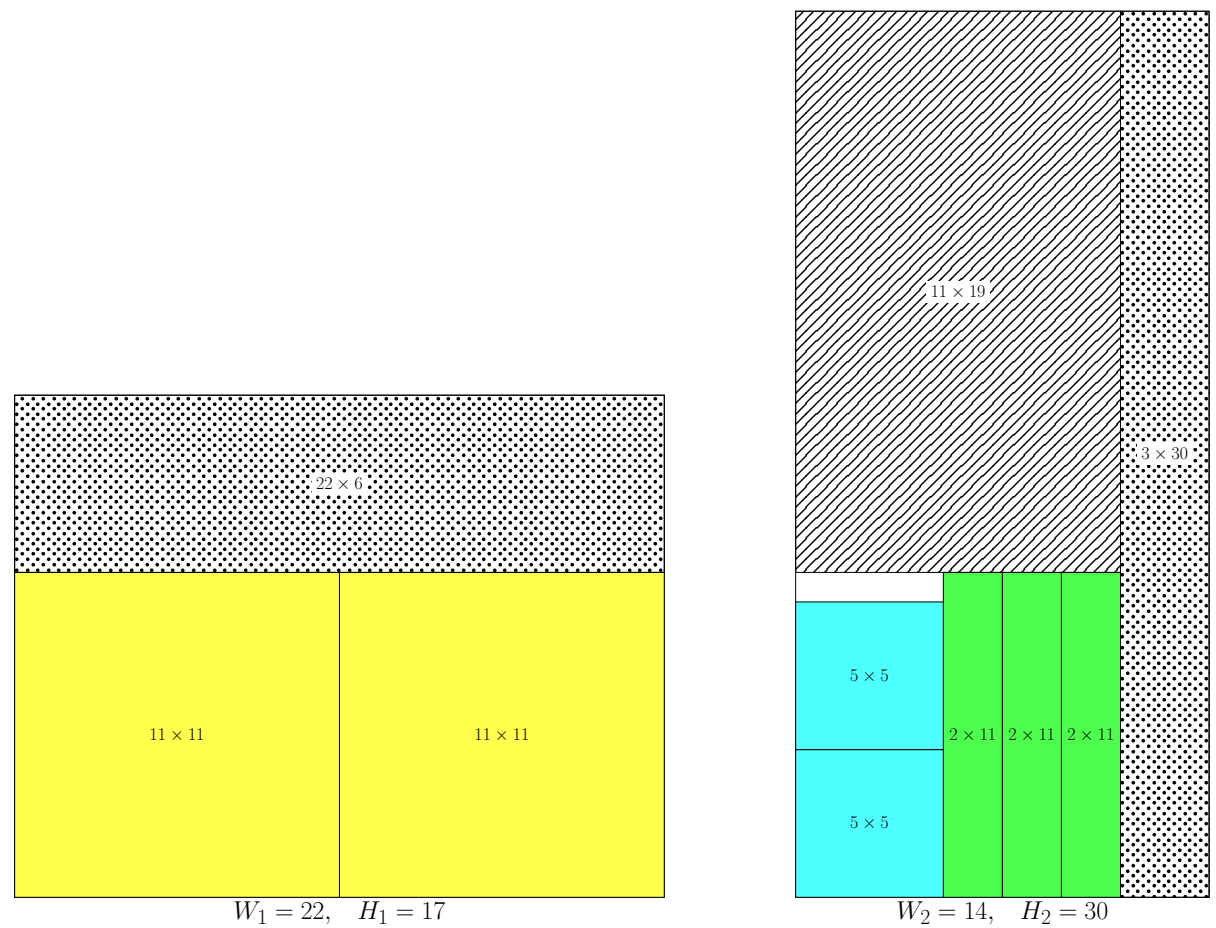

(6)
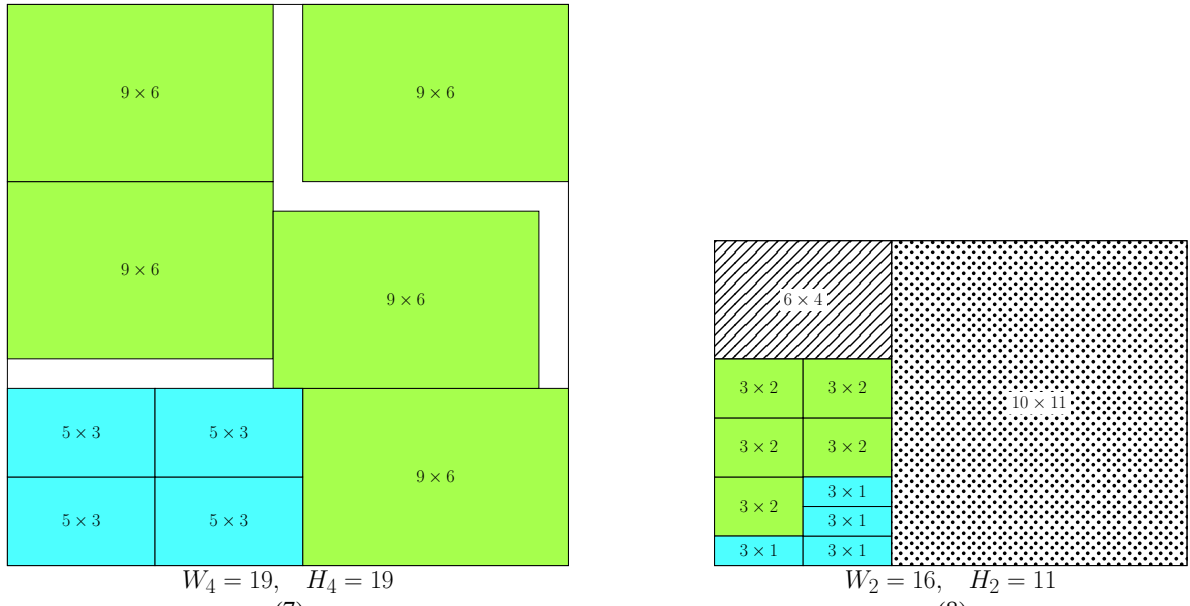

(8)

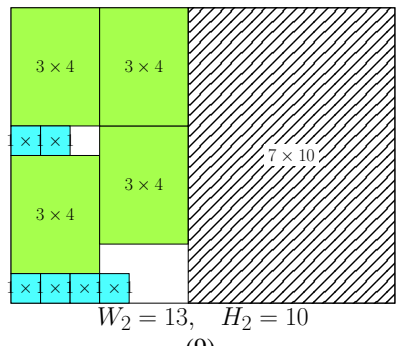

(9)

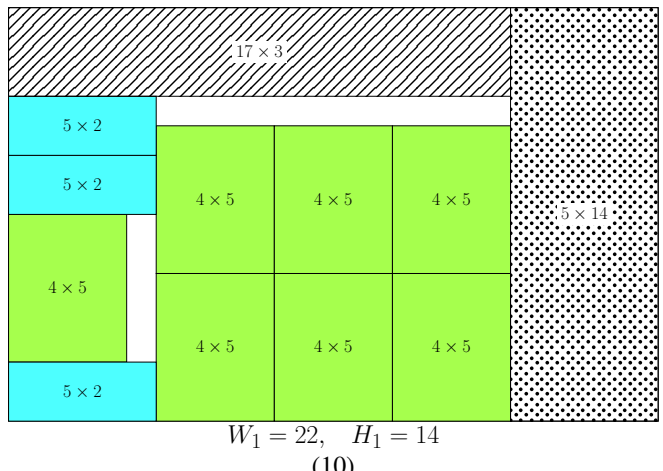

Figura 5.5: Ilustrações das soluções obtidas pelo modelo $\mathcal{S}_{0}^{G}$ para as instâncias 6,7,8,9 e 10 


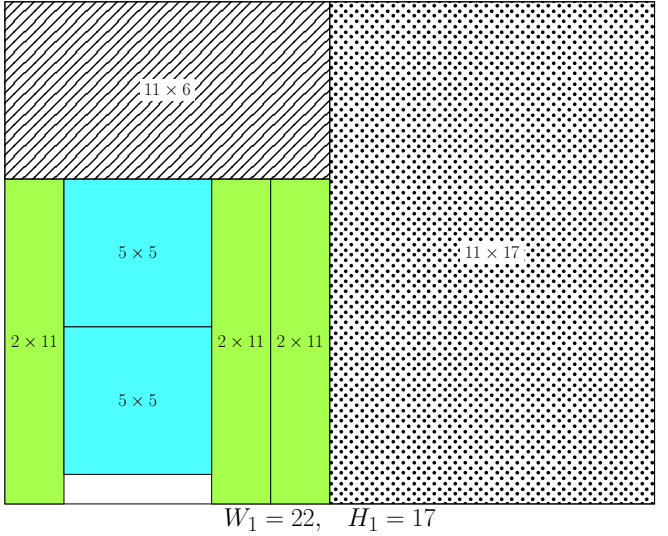

(1)

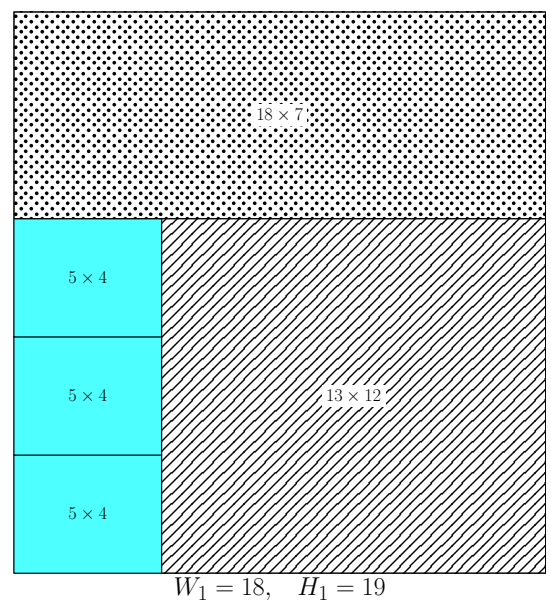

(3)

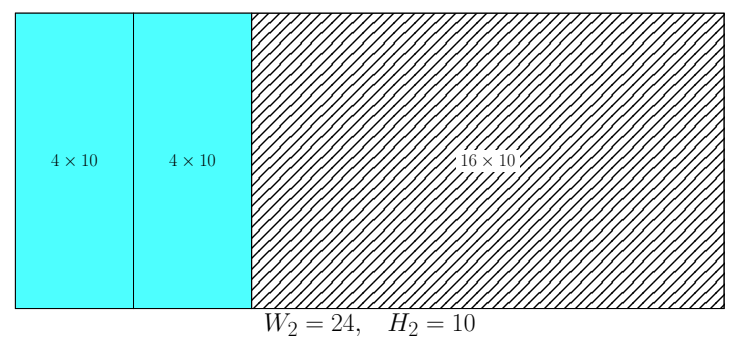

(2)

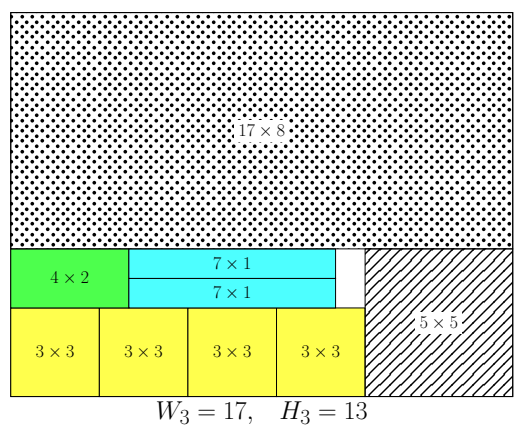

(4)

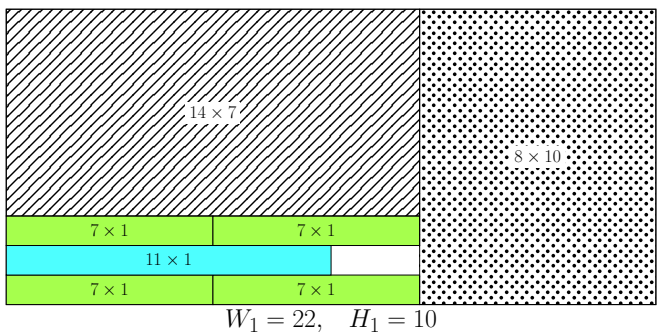

(5)

Figura 5.6: Ilustrações das soluções obtidas pelo modelo $\mathcal{S}_{1}^{G}$ para as instâncias 1, 2, 3, 4 e 5 . 


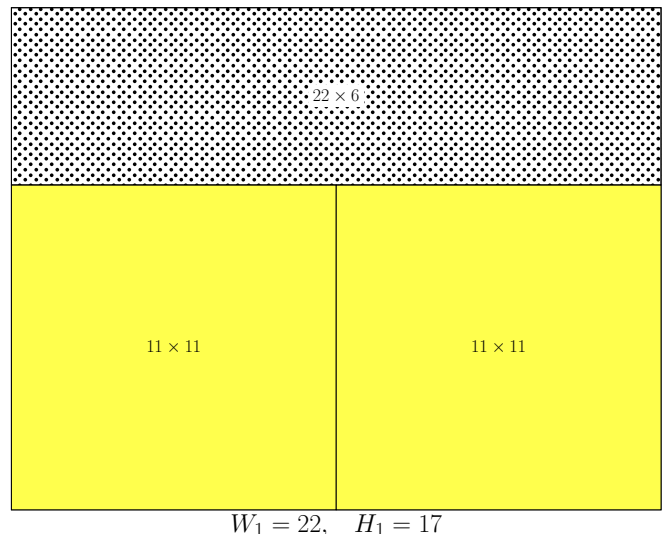

(6)
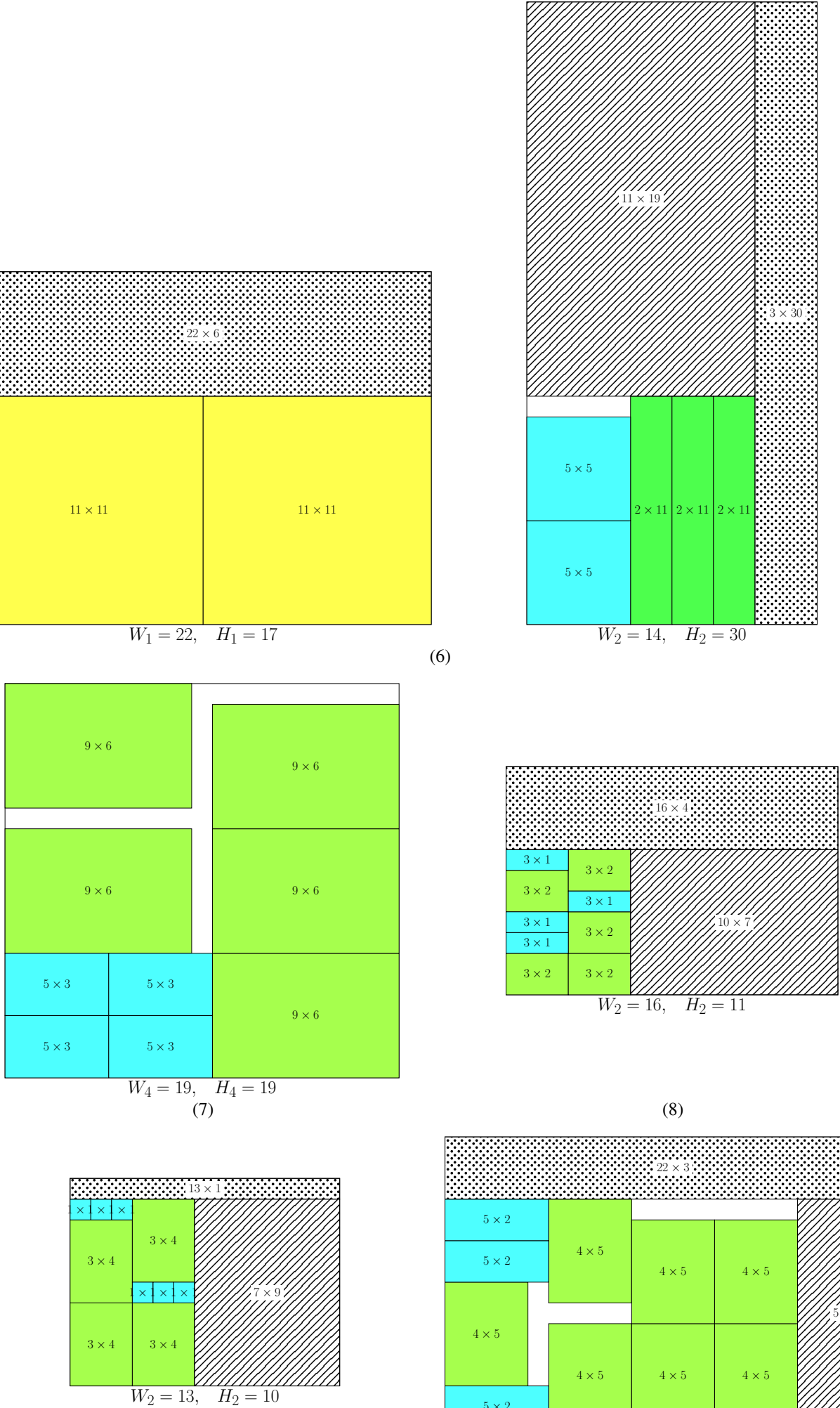

(9)

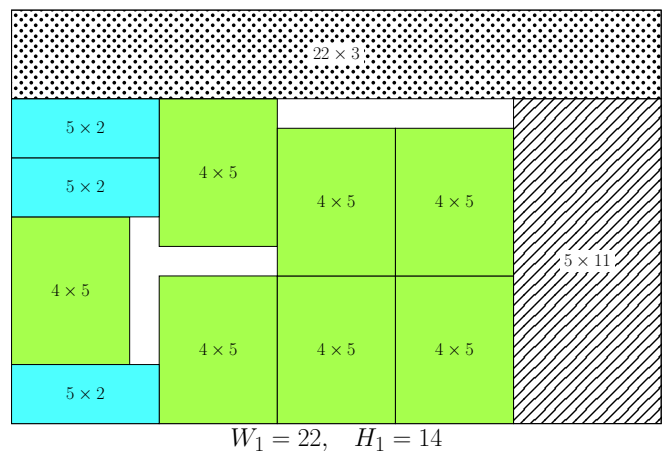

(10)

Figura 5.7: Ilustrações das soluções obtidas pelo modelo $\mathcal{S}_{1}^{G}$ para as instâncias 6,7,8,9 e 10. 


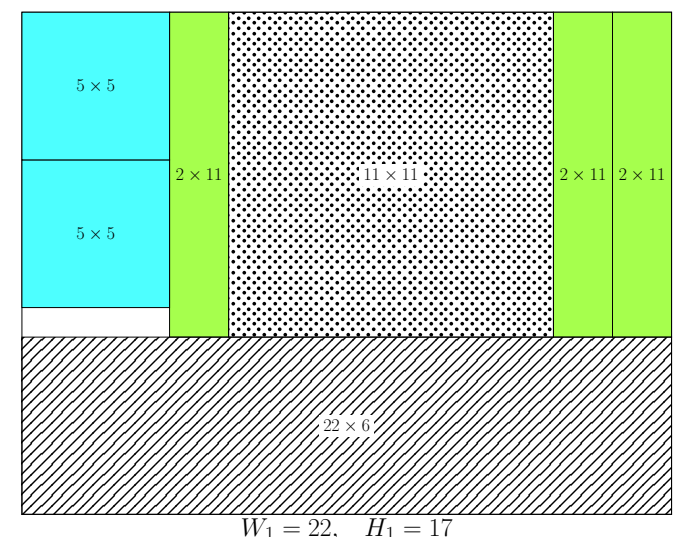

(1)

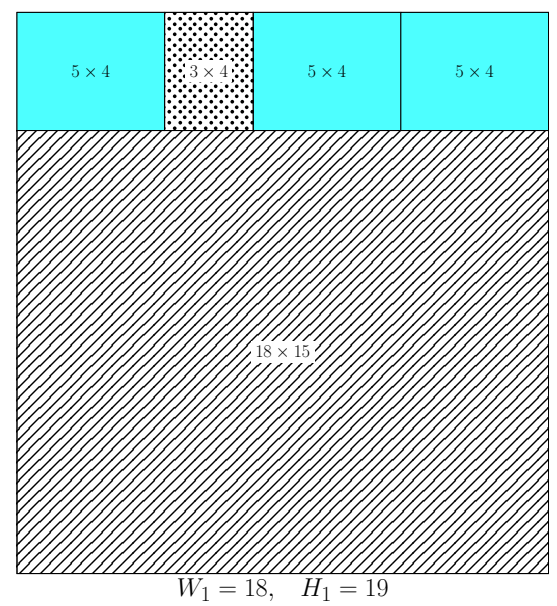

(3)

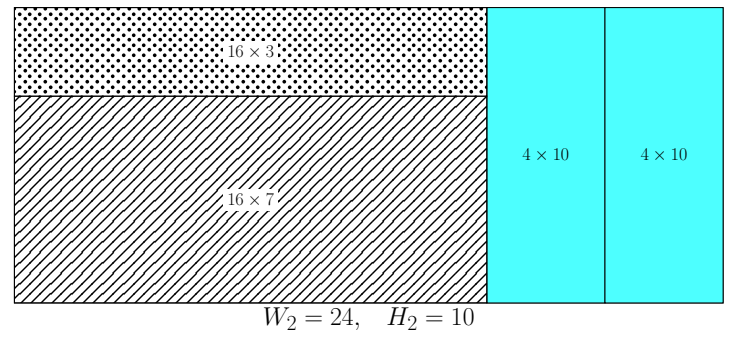

(2)

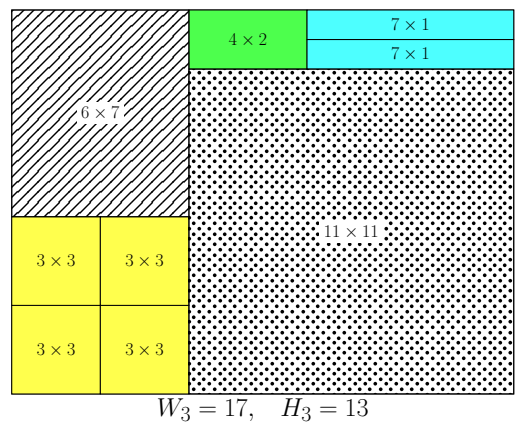

(4)

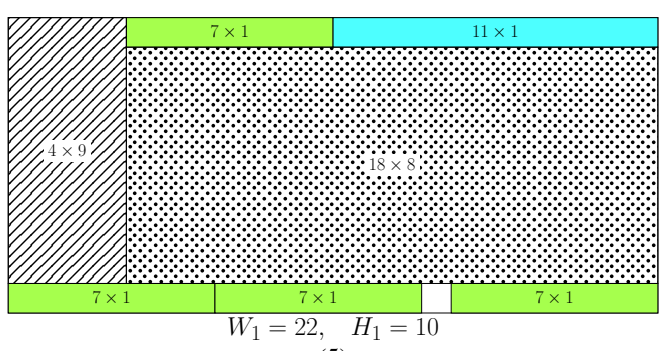

(5)

Figura 5.8: Ilustrações das soluções obtidas pelo modelo $\mathcal{S}_{2}^{G}$ para as instâncias 1, 2, 3, 4 e 5.

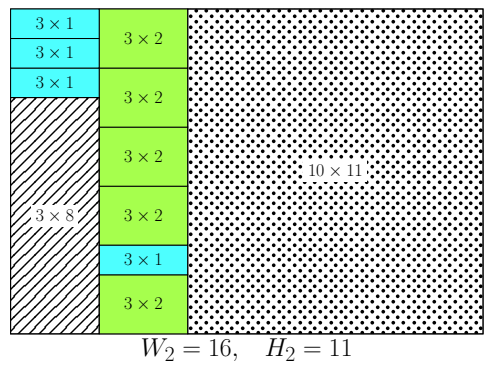

(8)

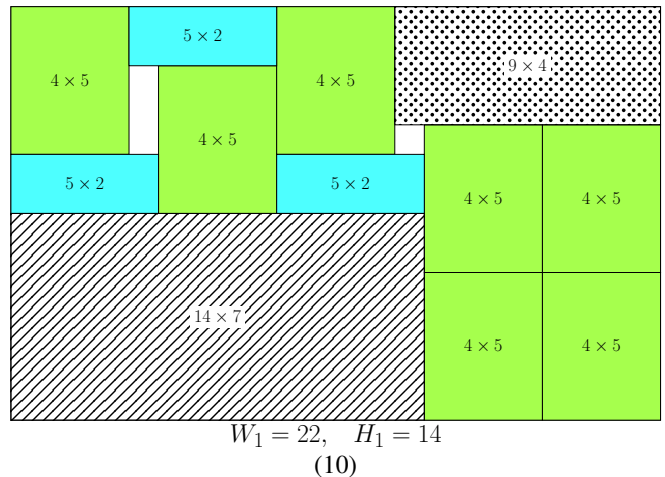

(10)

Figura 5.9: Ilustrações das soluções obtidas pelo modelo $\mathcal{S}_{2}^{G}$ para as instâncias 8 e 10. 


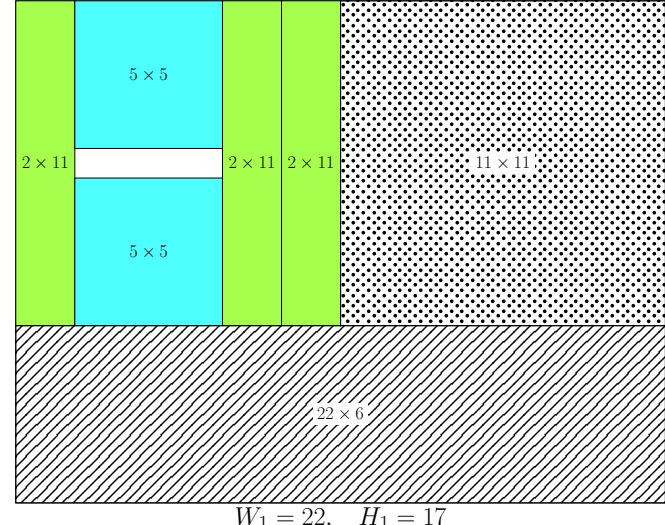

(1)

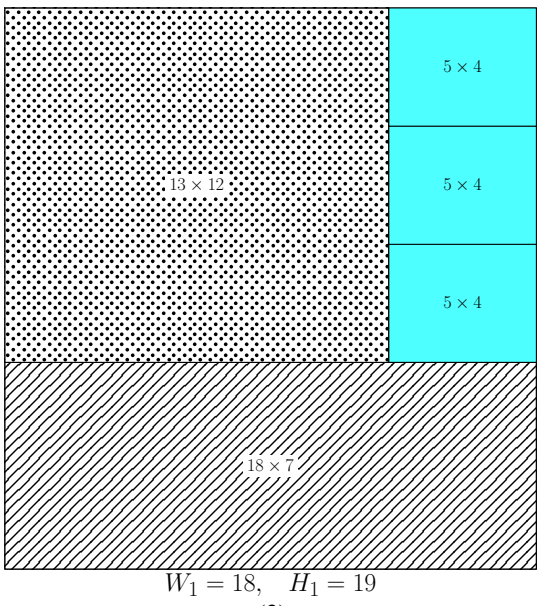

(3)

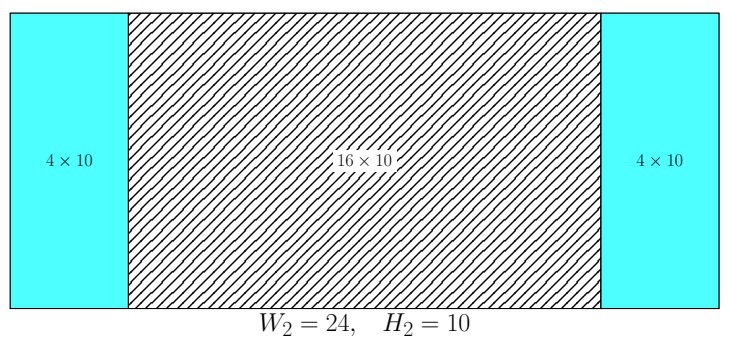

(2)

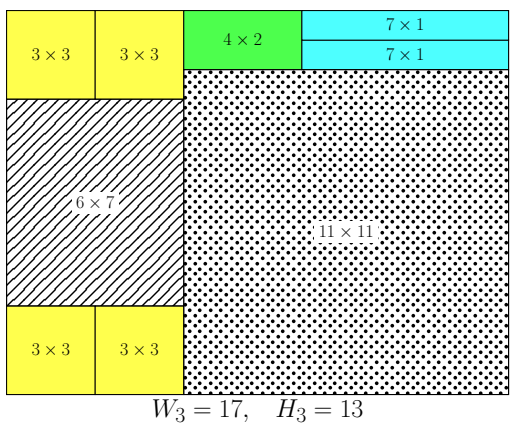

(4)

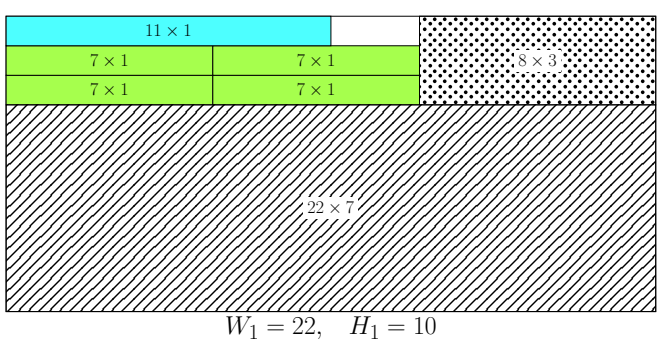

(5)

Figura 5.10: Ilustrações das soluções obtidas pelo modelo $\mathcal{S}_{3}^{G}$ para as instâncias 1, 2, 3, 4 e 5 . 


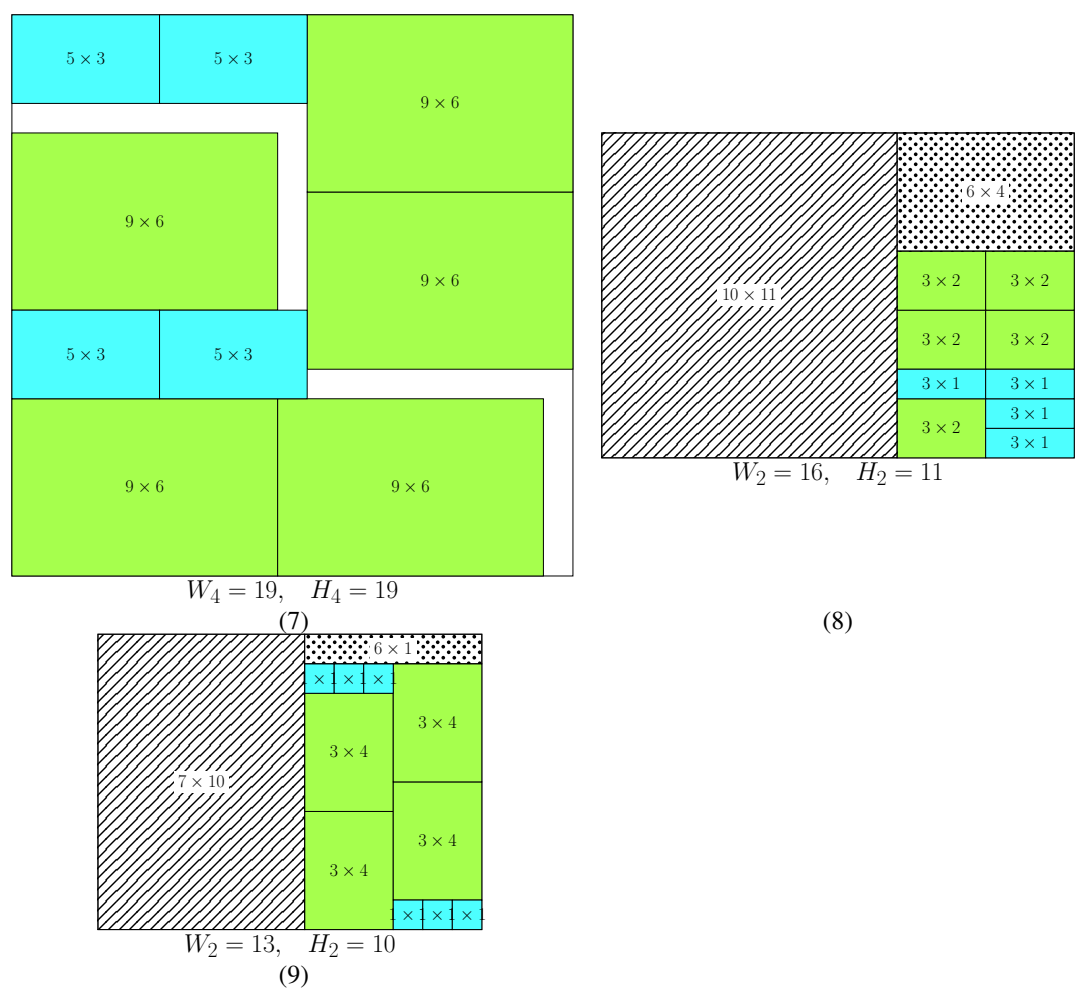

Figura 5.11: Ilustrações das soluções obtidas pelo modelo $\mathcal{S}_{3}^{G}$ para as instâncias 7,8 e 9 . 


\subsection{Estratégia de duas fases}

Considerando os modelos de programação em dois níveis (4.115), (4.116), (4.154) e (4.155), sabemos que o problema do nível inferior é sempre independente do problema do nível superior. Portanto, uma forma de resolver tais modelos de programação em dois níveis seria resolver inicialmente o problema do nível inferior e em seguida, resolver o problema do nível superior acrescido das restrições do problema do nível inferior mais uma restrição forçando o valor da função objetivo do nível inferior ser igual ao valor ótimo encontrado. Descreveremos, a seguir, essa estratégia baseando-se não dos modelos em dois níveis, mas nos modelos $\mathcal{S}_{i}^{G}$, com $i=\{0,1,2,3\}$. Chamamos essa estratégia de estratégia de duas fases, que pode ser descrita da seguinte forma:

1. Resolver o modelo (4.14-4.27) obtendo uma solução que minimiza o custo dos objetos utilizados, com valor $\sum_{l=1}^{p} u_{l}^{*} c_{l}$.

2. Acrescentar ao modelo $\mathcal{S}_{i}^{G}$ a restrição

$$
\sum_{l=1}^{p} u_{l} c_{l}=\sum_{l=1}^{p} u_{l}^{*} c_{l}
$$

e resolvê-lo.

É importante notar que ao inserir uma restrição do tipo (5.17) nos modelos $\mathcal{S}_{i}^{G}, \operatorname{com} i=\{0,1,2,3\}$, transformamos as funções objetivo dos modelos de forma que as únicas variáveis são aquelas que modelam o valor das sobras aproveitáveis. Assim, as funções objetivos poderiam ser reescritas sem as somatórias $\sum_{l=1}^{p} u_{l} c_{l}$ e sem a normalização dos valores das sobras. Optamos por não fazer isso para poder comparar de forma mais simples os valores obtidos como solução do procedimento de duas fases com outros experimentos realizados neste capítulo e no Capítulo 4.

\subsubsection{Experimentos}

Nesta seção apresentaremos alguns experimentos com a estratégia de duas fases. Modelamos o problema de corte de itens retangulares de forma a minimizar o custo dos objetos conforme (4.144.27), porém considerando as restrições (5.13-5.14) no lugar de (4.19-4.22), quando modelando a não sobreposição de dois itens do mesmo tipo. Chamamos este modelo de $\mathcal{M}^{M C}$. A Tabela 5.2 sumariza os experimentos numéricos com o modelo $\mathcal{M}^{M C}$. Observe que o esforço computacional realizado pelo CPLEX é quase desprezível na maioria das instância, com exceção das instâncias 6 e 7.

\begin{tabular}{|c||rc|r|r|r|r|}
\hline \multicolumn{7}{|c|}{ Modelo $\mathcal{M}^{M C}$} \\
\hline \multirow{2}{*}{ \# Instância } & \multicolumn{3}{c|}{ Dados das soluções } & \multicolumn{3}{c|}{ Medidas de desempenho } \\
\cline { 2 - 7 } & Lim. Inf. & Lim. Sup. & Placas Usadas & Iterações MIP & Nós B\&B & Tempo (s) \\
\hline 1 & 374 & 374 & 1 & 24 & 0 & 0.00 \\
2 & 240 & 240 & 1 & 10 & 0 & 0.00 \\
3 & 342 & 342 & 1 & 12 & 0 & 0.00 \\
4 & 221 & 221 & 1 & 43 & 0 & 0.00 \\
5 & 220 & 220 & 1 & 23 & 0 & 0.00 \\
6 & 794 & 794 & 2 & 24738 & 5673 & 0.59 \\
7 & 361 & 361 & 1 & 10758493 & 936596 & 92.63 \\
8 & 176 & 176 & 1 & 61 & 0 & 0.01 \\
9 & 130 & 130 & 1 & 48 & 0 & 0.01 \\
10 & 308 & 308 & 1 & 67 & 0 & 0.01 \\
\hline
\end{tabular}

Tabela 5.2: Resultados dos experimentos com os modelos $\mathcal{M}^{M C}$. 
Em seguida, para cada instância, modificamos os modelos $\mathcal{S}_{0}^{G}, \mathcal{S}_{1}^{G}, \mathcal{S}_{2}^{G}$ e $\mathcal{S}_{3}^{G}$ de forma a possuírem uma restrição adicional na forma de (5.17). Para cada instância, consideramos como $\sum_{l=1}^{p} u_{l}^{*} c_{l}$ os valores na coluna "Lim. Sup." da Tabela 5.2. Os resultados dos experimentos com os modelos acrescidos dessa restrição encontram-se na Tabela 5.3. Em comparação com os valores já apresentados na Tabela 5.1, observamos que os resultados obtidos com o modelo $\mathcal{S}_{0}^{G}$ foram muito similares, com uma pequena vantagem para a estratégia de duas fases nas instâncias 1,2,5,6,8 e 9. Com relação ao modelo $\mathcal{S}_{1}^{G}$, temos também resultados muito similares, com uma vantagem pequena da estratégia de duas fases nas instâncias 1,2,3,5,6,9 e 10. Com relação ao modelo $\mathcal{S}_{2}^{G}$ o desempenho foi muito similar nos dois experimentos. A estratégia de duas fases teve um desempenho quase três vezes pior na instância 10 . Com relação ao modelo $\mathcal{S}_{3}^{G}$ o desempenho da estratégia de duas fases foi consideravelmente pior nas instâncias 7 e 8 que não foram resolvidas dentro do limite de tempo de 21600 segundos. Por outro lado, conseguimos resolver a instância 10 que antes não era resolvida com o modelo $\mathcal{S}_{3}^{G}$. Note que o valor das sobras é maior que o obtido para a instância 10 com o modelo $\mathcal{S}_{2}^{G}$. A solução obtida para esta instância pode ser visualizada na Figura 5.12.

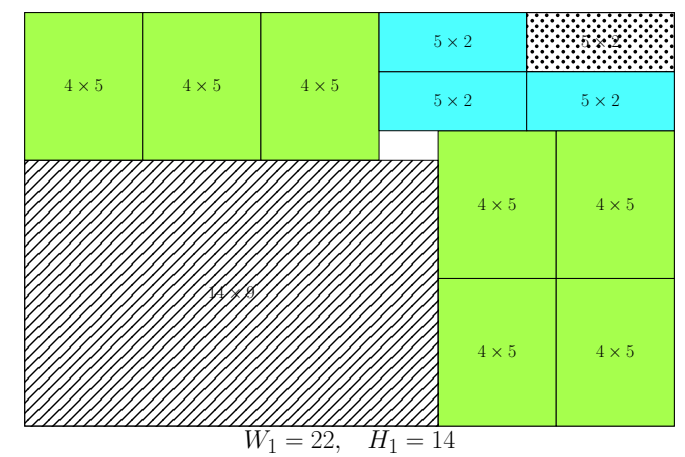

Figura 5.12: Ilustrações da solução obtida pelo modelo $\mathcal{S}_{3}^{G}$ acrescido de restrição na forma (5.17) para a instância 10. 


\begin{tabular}{|c|c|c|c|c|c|c|c|}
\hline \multicolumn{8}{|c|}{ Modelo $\mathcal{S}_{0}^{G}$} \\
\hline \multirow[t]{2}{*}{ \# Instância } & \multicolumn{4}{|c|}{ Dados das soluções } & \multicolumn{3}{|c|}{ Medidas de desempenho } \\
\hline & Lim. Inf. & Lim. Sup. & V. Sobras & Placas Usadas & Iterações MIP & Nós B\&B & Tempo (s) \\
\hline 1 & 373,700824596226 & 373,700824596226 & 94622 & 1 & 224 & 70 & 0,10 \\
\hline 2 & 239,872276308918 & 239,872276308918 & 38400 & 1 & 52 & 0 & 0,02 \\
\hline 3 & 341,782856165062 & 341,782856165062 & 96444 & 1 & 122 & 21 & 0,06 \\
\hline 4 & 220,826167037155 & 220,826167037155 & 35581 & 1 & 13272 & 4898 & 0,57 \\
\hline 5 & 219,768973003587 & 219,768973003587 & 39160 & 1 & 464 & 156 & 0,13 \\
\hline 6 & 793,446850219429 & 793,446850219429 & 174948 & 2 & 314478 & 120132 & 7,81 \\
\hline 7 & 361,000000000000 & 361,000000000000 & 0 & 1 & 1401 & 198 & 0,39 \\
\hline 8 & 175,825697498245 & 175,825697498245 & 23584 & 1 & 253826 & 86678 & 7,12 \\
\hline 9 & 129,937883959044 & 129,937883959044 & 9100 & 1 & 3427 & 808 & 0,30 \\
\hline 10 & 307,851923076923 & 307,851923076923 & 37268 & 1 & 212376 & 71713 & 5,02 \\
\hline \multicolumn{8}{|c|}{ Modelo $\mathcal{S}_{1}^{G}$} \\
\hline \multirow[t]{2}{*}{ \# Instância } & \multicolumn{4}{|c|}{ Dados das soluções } & \multicolumn{3}{|c|}{ Medidas de desempenho } \\
\hline & Lim. Inf. & Lim. Sup. & V. Sobras & Placas Usadas & Iterações MIP & Nós B\&B & Tempo (s) \\
\hline 1 & 373,700824596226 & 373,700824596226 & 94622 & 1 & 247 & 74 & 0,08 \\
\hline 2 & 239,872276308918 & 239,872276308918 & 38400 & 1 & 57 & 0 & 0,01 \\
\hline 3 & 341,782856165062 & 341,782856165062 & 96444 & 1 & 114 & 17 & 0,08 \\
\hline 4 & 220,826167037155 & 220,826167037155 & 35581 & 1 & 12292 & 4531 & 0,57 \\
\hline 5 & 219,768973003587 & 219,768973003587 & 39160 & 1 & 690 & 187 & 0,14 \\
\hline 6 & 793,446850219429 & 793,446850219429 & 174948 & 2 & 511113 & 212459 & 15,45 \\
\hline 7 & 361,000000000000 & 361,000000000000 & 0 & 1 & 1142 & 87 & 0,39 \\
\hline 8 & 175,825697498245 & 175,825697498245 & 23584 & 1 & 360054 & 119087 & 10,05 \\
\hline 9 & 129,932559726962 & 129,932559726962 & 9880 & 1 & 18714 & 4501 & 0,61 \\
\hline 10 & 307,851922867614 & 307,851922867614 & 37268 & 1 & 149771 & 56000 & 4,82 \\
\hline \multicolumn{8}{|c|}{ Modelo $\mathcal{S}_{2}^{G}$} \\
\hline \multirow[t]{2}{*}{ \# Instância } & \multicolumn{4}{|c|}{ Dados das soluções } & \multicolumn{3}{|c|}{ Medidas de desempenho } \\
\hline & Lim. Inf. & Lim. Sup. & V. Sobras & Placas Usadas & Iterações MIP & Nós B\&B & Tempo (s) \\
\hline 1 & 373,700824596226 & 373,700824596226 & 94622 & 1 & 66632 & 18461 & 1,99 \\
\hline 2 & 239,872276308918 & 239,872276308918 & 38400 & 1 & 876 & 197 & 0,14 \\
\hline 3 & 341,782856165062 & 341,782856165062 & 96444 & 1 & 8541 & 1842 & 0,36 \\
\hline 4 & 220,824007621461 & 220,824007621461 & 36023 & 1 & 7216177 & 1451858 & 125,17 \\
\hline 5 & 219,766377090331 & 219,766377194615 & 39600 & 1 & 500177 & 68728 & 6,15 \\
\hline 6 & 793,370454919121 & 793,440937662042 & - & - & - & - & $\geq 21600,00$ \\
\hline 7 & 360,984798951039 & 361,000000000000 & - & - & - & - & $\geq 21600,00$ \\
\hline 8 & 175,825697354172 & 175,825697354172 & 23584 & 1 & 446771989 & 76293985 & 7018,88 \\
\hline 9 & 129,930784982932 & 129,932559726957 & - & - & - & - & $\geq 21600,00$ \\
\hline 10 & 307,835986172899 & 307,836013986003 & 41272 & 1 & 875786725 & 146103618 & 13348,25 \\
\hline \multicolumn{8}{|c|}{ Modelo $\mathcal{S}_{3}^{G}$} \\
\hline \multirow[t]{2}{*}{ \# Instância } & \multicolumn{4}{|c|}{ Dados das so } & \multicolumn{3}{|c|}{ Medidas de desempenho } \\
\hline & Lim. Inf. & Lim. Sup. & V. Sobras & Placas Usadas & Iterações MIP & Nós B\&B & Tempo (s) \\
\hline 1 & 373,700824596239 & 373,700824596239 & 94622 & 1 & 80793 & 27607 & 2,95 \\
\hline 2 & 239,872276308918 & 239,872276308918 & 38400 & 1 & 1096 & 290 & 0,15 \\
\hline 3 & 341,782856165062 & 341,782856165062 & 96444 & 1 & 6250 & 1829 & 0,36 \\
\hline 4 & 220,823993665179 & 220,824007621449 & 36023 & 1 & 7765083 & 1318199 & 130,16 \\
\hline 5 & 219,768973003587 & 219,768973003587 & 39160 & 1 & 595521 & 110211 & 9,51 \\
\hline 6 & 793,262150779696 & 793,440937662041 & - & - & - & - & $\geq 21600,00$ \\
\hline 7 & 360,983109945599 & 361,000000000000 & - & - & - & - & $\geq 21600,00$ \\
\hline 8 & 175,824396733306 & 175,825697498241 & - & - & - & - & $\geq 21600,00$ \\
\hline 9 & 129,932559709069 & 129,932559726969 & 9880 & 1 & 107407294 & 13945613 & 1457,72 \\
\hline 10 & 307,833536585362 & 307,833566433549 & 41888 & 1 & 795619037 & 117321914 & 10832,49 \\
\hline
\end{tabular}

Tabela 5.3: Resultados dos experimentos com os modelos $\mathcal{S}_{0}^{G}, \mathcal{S}_{1}^{G}, \mathcal{S}_{2}^{G}$ e $\mathcal{S}_{3}^{G}$, acrescidos de restrições na forma de na forma de (5.17). 


\subsection{Minimizando a quantidade de sobras aproveitáveis}

Nesta seção apresentamos uma forma de modelar uma variação do problema de corte com sobras aproveitáveis, na qual deseja-se obter, dentre as soluções que minimizam o custo dos objetos, aquelas que maximizam o custo das sobras e, dentre estas últimas, uma que minimize a quantidade de sobras aproveitáveis. Modelos para esta variação do problema podem ser considerados uma melhoria aos modelos já apresentados para o problema de corte com sobras, uma vez que buscam obter soluções com quantidade mínima de sobras. Assim, este novo problema pode ser interpretado como uma forma de eliminar soluções equivalentes do problema de corte com sobras aproveitáveis. Porem neste caso o ganho seria qualitativo (soluções com menor quantidade de sobras aproveitáveis) e não quantitativo (resolver instâncias mais rapidamente).

Este problema pode ser modelado como um problema em três níveis, no qual no primeiro nível busca-se minimizar a quantidade de sobras aproveitáveis, no segundo nível busca-se maximizar o valor das sobras aproveitáveis e no terceiro nível busca-se minimizar o custo dos objetos utilizados. Da mesma forma que nossos modelos de dois níveis apresentados anteriormente, o problema do terceiro nível é independente do problema do segundo nível e o problema do segundo nível é independentes do problema do primeiro nível. Isso nos permite modelar tal problema como um MIP que contem todas as restrições de todos os níveis e cuja função objetivo é uma soma ponderada das funções objetivos de cada nível. Podemos também resolver esse problema em três níveis através de uma estratégia similar a apresentada na Seção 5.2, só que agora utilizando três fases ao invés de duas. A seguir apresentaremos modelos MIPs para este problema, bem como detalharemos a estratégia em três fases para resolvê-lo.

Para minimizar a quantidade de sobras aproveitáveis é necessário contabilizá-las. Considerando as sobras aproveitáveis definidas a partir de um par de dimensões mínimas, ou seja, considerando os modelos $\mathcal{S}_{0}^{G}$ e $\mathcal{S}_{2}^{G}$, podemos contabilizar as sobras através das variáveis $z_{l}$ e $\bar{z}_{l}$. Para cada $z_{l}=0$ ou $\bar{z}_{l}=0$ temos uma sobra aproveitável no objeto $l$. Assim, podemos contabilizar as sobras aproveitáveis através da expressão:

$$
\sum_{l=1}^{p}\left(1-z_{l}\right)+\left(1-\bar{z}_{l}\right) .
$$

Considerando as sobras aproveitáveis definidas a partir da lista de dimensões de itens demandados, ou seja, da forma feita nos modelos $\mathcal{S}_{1}^{G}$ e $\mathcal{S}_{3}^{G}$ podemos contabilizar as sobras através das variáveis $\tau_{i l}$ e $\bar{\tau}_{i l}$. Lembrando que $\tau_{i l}=0$ se o i-ésimo item da lista cabe na primeira sobra do objeto $l$ e $\tau_{i l}=1$ se não cabe. O mesmo vale para $\bar{\tau}_{i l}$ em relação ao i-ésimo item e a segunda sobra do objeto $l$. Temos também que se $\tau_{i l}=n$ então a primeira sobra do objeto $l$ não é aproveitável e se $\bar{\tau}_{i l}=n$, a segunda sobra do objeto $l$ não é aproveitável. Considerando as novas variáveis $\rho_{l}$ e $\bar{\rho}_{l} \in[0,1]$, desejamos que $\rho_{l}=0$ caso a primeira sobra do objeto $l$ seja aproveitável e que $\bar{\rho}_{l}=0$ se a segunda sobra do objeto for aproveitável. Para isso, consideramos as seguintes restrições:

$$
\begin{aligned}
& \rho_{l} \leq \tau_{i l}, \\
& \bar{\rho}_{l} \leq \bar{\tau}_{i l},
\end{aligned}
$$

para $i=1, \ldots, n$ e $l=1, \ldots, p$. Assim, (5.19) força $\rho_{l}=0$ caso a primeira sobra do objeto $l$ for aproveitável. Similarmente, (5.20) força $\bar{\rho}_{l}=0$ caso a segunda sobra do objeto $l$ for aproveitável. Para minimizar a quantidade de sobras aproveitáveis basta minimizar:

$$
\sum_{l=1}^{p}\left(1-\rho_{l}\right)+\left(1-\bar{\rho}_{l}\right) .
$$


Observe que se $\sum_{i=1}^{n} \tau_{i l}=n$ (ou $\sum_{i=1}^{n} \bar{\tau}_{i l}=n$ ) para algum objeto $l$, então $\rho_{l}$ (ou $\bar{\rho}_{l}$ ) valerá 1 .

Podemos agora construir os modelos para as diferentes variações do problema de corte com quantidade mínima de sobras dentre aquelas de valor máximo. Consideraremos os casos com sobras guilhotinadas e sobras genéricas, usando tando um par de dimensões mínimas como uma lista de dimensões de itens para definir as sobras aproveitáveis. Definimos $\omega_{1}=1+\sum_{l=1}^{p} c_{l} W_{l} H_{l}$ e $\omega_{2}=1+2 p$. Considerando a definição de sobra aproveitável a partir de um par de dimensões mínimas, podemos construir os modelos para os casos de sobras guilhotinadas e sobras genéricas tomando como base os modelos $\mathcal{S}_{0}^{G}$ e $\mathcal{S}_{2}^{G}$, respectivamente. Para o problema envolvendo sobras guilhotinadas, construímos o modelo $\mathcal{Q}_{0}^{G}$ que consiste no modelo $\mathcal{S}_{0}^{G}$ substituindo-se a função objetivo a ser minimizada por:

$$
\omega_{1} \omega_{2} \sum_{l=1}^{p} u_{l} c_{l}-\omega_{1} \sum_{l=1}^{p}\left(A_{l}+B_{l}+\bar{A}_{l}+\bar{B}_{l}\right) c_{l}+\sum_{l=1}^{p}\left(1-z_{l}\right)+\left(1-\bar{z}_{l}\right) .
$$

Para o problema envolvendo sobras genéricas, construímos o modelo $\mathcal{Q}_{2}^{G}$ que consiste no modelo $\mathcal{S}_{2}^{G}$ substituindo-se a função objetivo a ser minimizada por:

$$
\omega_{1} \omega_{2} \sum_{l=1}^{p} u_{l} c_{l}-\omega_{1} \sum_{l=1}^{p}\left(A_{l}+B_{l}\right) c_{l}+\sum_{l=1}^{p}\left(1-z_{l}\right)+\left(1-\bar{z}_{l}\right) .
$$

Considerando a definição de sobra aproveitável a partir de uma lista de dimensões de itens, podemos construir os modelos para os casos de sobras guilhotinadas e sobras genéricas tomando como base os modelos $\mathcal{S}_{1}^{G}$ e $\mathcal{S}_{3}^{G}$, respectivamente. Para o problema envolvendo sobras guilhotinadas, construímos o modelo $\mathcal{Q}_{1}^{G}$ que consiste no modelo $\mathcal{S}_{1}^{G}$ mais as restrições (5.19-5.20) e com sua função objetivo a ser minimizada substituída por:

$$
\omega_{1} \omega_{2} \sum_{l=1}^{p} u_{l} c_{l}-\omega_{1} \sum_{l=1}^{p}\left(A_{l}+B_{l}+\bar{A}_{l}+\bar{B}_{l}\right) c_{l}+\sum_{l=1}^{p}\left(1-\rho_{l}\right)+\left(1-\bar{\rho}_{l}\right) .
$$

Para o problema envolvendo sobras genéricas, construímos o modelo $\mathcal{Q}_{3}^{G}$ que consiste no modelo $\mathcal{S}_{3}^{G}$ mais as restrições (5.19-5.20) e com sua função objetivo a ser minimizada substituída por:

$$
\omega_{1} \omega_{2} \sum_{l=1}^{p} u_{l} c_{l}-\omega_{1} \sum_{l=1}^{p}\left(A_{l}+B_{l}\right) c_{l}+\sum_{l=1}^{p}\left(1-\rho_{l}\right)+\left(1-\bar{\rho}_{l}\right) .
$$

Podemos também resolver o problema de corte com sobras aproveitáveis e quantidade mínima de sobras através de uma estratégia em três fases. Considerando os modelos $\mathcal{S}_{i}^{G}$ e $\mathcal{Q}_{i}^{G}$, com $i=$ $\{0,1,2,3\}$, a estratégia de solução em três fases, pode ser descrita da seguinte forma:

1. Resolver o modelo $\mathcal{M}^{M C}$ obtendo uma solução que minimiza o custo dos objetos utilizados, com valor $\sum_{l=1}^{p} u_{l}^{*} c_{l}$.

2. Acrescentar ao modelo $\mathcal{S}_{i}^{G}$ a restrição

$$
\sum_{l=1}^{p} u_{l} c_{l}=\sum_{l=1}^{p} u_{l}^{*} c_{l}
$$

e resolvê-lo, obtendo o valor ótimo para a maximização das sobras aproveitáveis. 
3. Acrescentar ao modelo $\mathcal{Q}_{i}^{G}$ a restrição (5.26) e a restrição

$$
\sum_{l=1}^{p}\left(A_{l}+B_{l}+\bar{A}_{l}+\bar{B}_{l}\right) c_{l}=\sum_{l=1}^{p} c_{l}\left(A_{l}^{*}+B_{l}^{*}+\bar{A}_{l}^{*}+\bar{B}_{l}^{*}\right)
$$

caso $i=\{0,1\}$ ou a restrição

$$
\sum_{l=1}^{p}\left(A_{l}+B_{l}\right) c_{l}=\sum_{l=1}^{p} c_{l}\left(A_{l}^{*}+B_{l}^{*} \bar{A}_{l}^{*}+\bar{B}_{l}^{*}\right)
$$

caso $i=\{2,3\}$.

\subsubsection{Experimentos}

Implementamos os modelos MIPs $\mathcal{Q}_{0}^{G}, \mathcal{Q}_{1}^{G}, \mathcal{Q}_{2}^{G}$ e $\mathcal{Q}_{3}^{G}$, bem como os modelos para realização da estratégia em três fases. Todas implementações foram mais uma vez feitas utilizando-se o CPLEX 12.1 e a Concert Techonology 2.9. Os programas foram compilados utilizando o compilador G++ 4.4.3 da GCC (GNU Compiller Collection) e os experimentos realizados em uma máquina com 8GB de memória RAM, dois processadores de $2.6 \mathrm{GHz}$ Intel Xeon, com 6 núcleos cada um e tecnologia Hyper Threading. As opções de configuração do CPLEX, bem como, as constantes e demais valores relativos à caracterização das sobras nos modelos, são as mesmas utilizadas na seção4.4.

Consideramos as instâncias descritas na Tabela 4.3, bem como três novas instâncias descritas na Tabela 5.4. Os resultados obtidos com os modelos MIPs $\mathcal{Q}_{0}^{G}, \mathcal{Q}_{1}^{G}, \mathcal{Q}_{2}^{G}$ e $\mathcal{Q}_{3}^{G}$, e através da estratégia em três fases foram similares, assim vamos relatar em detalhes apenas os obtidos com os modelos MIPs. Os resultados dos experimentos com as instâncias 1-10 estão resumidos na Tabela 5.5 e os resultados com as instâncias 11-13 estão resumidos na Tabela 5.6. Nestas tabelas temos uma coluna adicional, a coluna "Qtde. Sobras" que reporta a quantidade de sobras aproveitáveis a solução possui. Considerando as instâncias 1-10 e comparando esta nova coluna com a quantidade de sobras observadas nas Figuras 5.4 - 5.11, podemos observar que a quantidade mínima de sobras já era atingida anteriormente, por coincidência, em praticamente todas as instâncias, com exceção da instância 2 no experimento com o modelo $\mathcal{S}_{2}^{G}$, como pode ser observado na Figura 5.8-(2). Considerando as instâncias 11-13, a Figura 5.13 ilustra três possíveis soluções sem considerar a minimização das sobras. Todas as soluções ilustradas nessa figura possuem duas sobras. Já a Tabela 5.6 mostra os resultados considerando a minimização de sobras. Não conseguimos resolver a instância 13 com os modelos $\mathcal{S}_{2}^{G}$ e $\mathcal{S}_{3}^{G}$, dentro do limite de tempo estipulado. Em todos os demais casos foi possível encontrar soluções que maximizam o valor das sobras aproveitáveis com apenas

\begin{tabular}{|c|c|c|c|c|}
\hline \multicolumn{5}{|c|}{ Descrição das Instâncias } \\
\hline \multirow{2}{*}{ \# } & \multicolumn{2}{|c|}{ Itens } & \multicolumn{2}{|c|}{ Objetos } \\
\hline & Qtde. Total & Qtde. Tipos & Qtde. Total & Qtde. Tipos \\
\hline \multirow[b]{2}{*}{11} & 7 & 3 & 2 & 1 \\
\hline & \multicolumn{2}{|c|}{$\begin{array}{l}3(3 \times 6) \\
2(6 \times 3) \\
2(3 \times 3)\end{array}$} & \multicolumn{2}{|c|}{$2(12 \times 18)$} \\
\hline \multirow{3}{*}{12} & 6 & 3 & 2 & 2 \\
\hline & \multicolumn{2}{|c|}{$3(1 \times 1)$} & \multicolumn{2}{|c|}{$1(6 \times 9)$} \\
\hline & & & ontinua na p & xima página \\
\hline
\end{tabular}
uma sobra. 
Tabela 5.4 - continuação da página anterior

\begin{tabular}{|c|c|c|c|c|}
\hline \multirow{2}{*}{ \# } & \multicolumn{2}{|c|}{ Itens } & \multicolumn{2}{|c|}{ Objetos } \\
\hline & Qtde. Total & Qtde. Tipos & Qtde. Total & Qtde. Tipos \\
\hline & \multicolumn{2}{|c|}{$\begin{array}{l}2(3 \times 2) \\
1(3 \times 1)\end{array}$} & \multicolumn{2}{|c|}{$1(3 \times 3)$} \\
\hline \multirow[b]{2}{*}{13} & 6 & 2 & 2 & 1 \\
\hline & \multicolumn{2}{|c|}{$\begin{array}{l}2(3 \times 1) \\
2(3 \times 3) \\
2(3 \times 4)\end{array}$} & \multicolumn{2}{|c|}{$2(6 \times 7)$} \\
\hline
\end{tabular}

Tabela 5.4: Descrição das instâncias 11, 12 e 13.

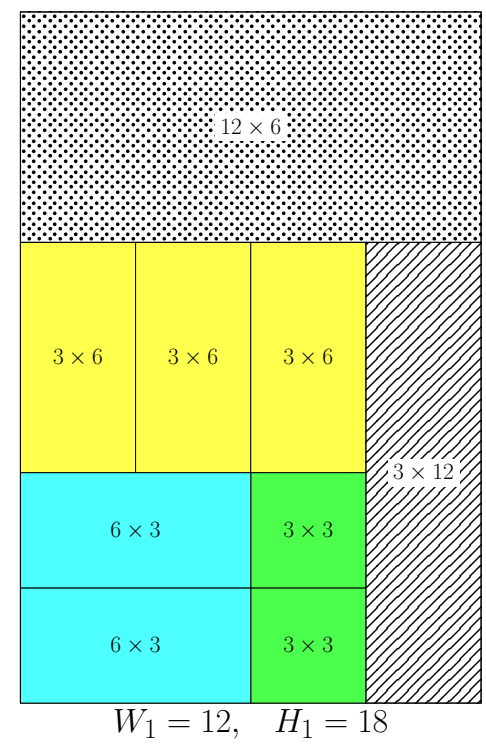

(11)

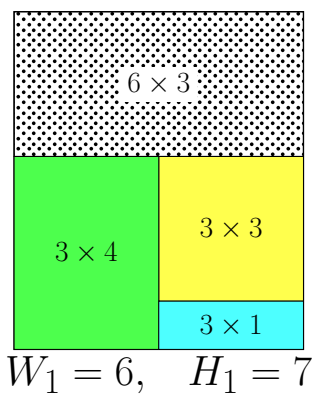

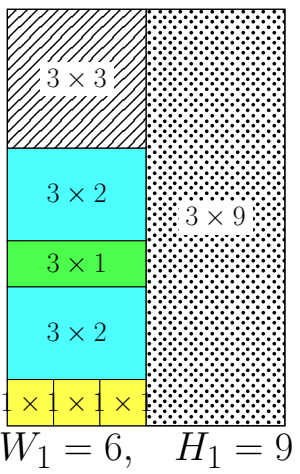

(12)

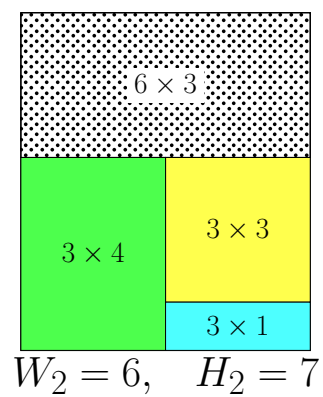

(13)

Figura 5.13: Padrões de corte com mesmo custo e valor de sobra que as encontradas para as instâncias 11,12 e 13, porém com quantidade de sobras superior à quantidade mínima. 


\begin{tabular}{|c|c|c|c|c|c|c|c|c|}
\hline \multicolumn{9}{|c|}{ Modelo $\mathcal{Q}_{0}^{G}$} \\
\hline \multirow[t]{2}{*}{ \# Instância } & \multicolumn{5}{|c|}{ Dados das soluções } & \multicolumn{3}{|c|}{ Medidas de desempenho } \\
\hline & Lim. Inf. & Lim. Sup. & V. Sobras & Qtde. Sobras & Placas Usadas & Iterações MIP & Nós $B \& B$ & Tempo (s) \\
\hline 1 & 590923741,999983 & 590964882 & 94622 & 2 & 1 & 278 & 56 & 0,21 \\
\hline 2 & 360588001,000000 & 360588001 & 38400 & 1 & 1 & 70 & 0 & 0,00 \\
\hline 3 & 758944171,999994 & 759012572 & 96444 & 2 & 1 & 113 & 1 & 0,10 \\
\hline 4 & 316369082,299999 & 316400177 & 35581 & 2 & 1 & 2882 & 703 & 0,28 \\
\hline 5 & 186244302,000000 & 186259702 & 39160 & 2 & 1 & 478 & 97 & 0,15 \\
\hline 6 & 1254619986,09524 & 1254744953 & 174948 & 3 & 2 & 3339687 & 836449 & 49,95 \\
\hline 7 & 3394680828,10388 & 3394974682 & 0 & 0 & 1 & 6053 & 782 & 0,54 \\
\hline 8 & 118940098,000000 & 118951362 & 23584 & 2 & 1 & 3490 & 797 & 0,38 \\
\hline 9 & 95174950,9999909 & 95180151 & 9100 & 1 & 1 & 268 & 3 & 0,22 \\
\hline 10 & 387364120,634921 & 387402402 & 37268 & 2 & 1 & 17976 & 6017 & 0,81 \\
\hline \multicolumn{9}{|c|}{ Modelo $\mathcal{Q}_{1}^{G}$} \\
\hline \multirow[t]{2}{*}{ \# Instância } & \multicolumn{5}{|c|}{ Dados das soluções } & \multicolumn{3}{|c|}{ Medidas de desempenho } \\
\hline & Lim. Inf. & Lim. Sup. & V. Sobras & Qtde. Sobras & Placas Usadas & Iterações MIP & Nós $B \& B$ & Tempo (s) \\
\hline 1 & 590923740,699959 & 590964882 & 94622 & 2 & 1 & 427 & 100 & 0,19 \\
\hline 2 & 360588000,500000 & 360588001 & 38400 & 1 & 1 & 76 & 0 & 0,00 \\
\hline 3 & 758967570,474381 & 759012572 & 96444 & 2 & 1 & 157 & 3 & 0,11 \\
\hline 4 & 316369080,554531 & 316400177 & 35581 & 2 & 1 & 15141 & 4523 & 0,62 \\
\hline 5 & 186242101,160000 & 186259702 & 39160 & 2 & 1 & 741 & 165 & 0,20 \\
\hline 6 & 1254619629,32176 & 1254744953 & 174948 & 3 & 2 & 13722156 & 3324172 & 217,58 \\
\hline 7 & 3394672886,01170 & 3394974682 & 0 & 0 & 1 & 10156 & 843 & 1,32 \\
\hline 8 & 118939685,502189 & 118951362 & 23584 & 2 & 1 & 6429 & 1855 & 0,56 \\
\hline 9 & 95169007,2474969 & 95176252 & 9880 & 2 & 1 & 3083 & 656 & 0,47 \\
\hline 10 & 387364374,057174 & 387402402 & 37268 & 2 & 1 & 322782 & 118541 & 9,55 \\
\hline \multicolumn{9}{|c|}{ Modelo $\mathcal{Q}_{2}^{G}$} \\
\hline \multirow{2}{*}{ \# Instância } & \multicolumn{5}{|c|}{ Dados das soluções } & \multicolumn{3}{|c|}{ Medidas de desempenho } \\
\hline & Lim. Inf. & Lim. Sup. & V. Sobras & Qtde. Sobras & Placas Usadas & Iterações MIP & Nós B\&B & Tempo (s) \\
\hline 1 & 590964881,352846 & 590964882 & 94622 & 2 & 1 & 78952 & 23834 & 2,29 \\
\hline 2 & 360588000,666667 & 360588001 & 38400 & 1 & 1 & 766 & 155 & 0,18 \\
\hline 3 & 759012571,999974 & 759012572 & 96444 & 2 & 1 & 7060 & 1987 & 0,31 \\
\hline 4 & 316397081,835877 & 316397083 & 36023 & 2 & 1 & 8623275 & 1724045 & 153,21 \\
\hline 5 & 186257501,040794 & 186257502 & 39160 & 2 & 1 & 118950 & 25448 & 2,61 \\
\hline 6 & & & - & - & - & - & - & $\geq 21600,00$ \\
\hline 7 & & & - & - & - & - & - & $\geq 21600,00$ \\
\hline 8 & 118951360,743799 & 118951362 & 23584 & 2 & 1 & 691705039 & 91202186 & 8810,39 \\
\hline 9 & & & - & - & - & - & - & $\geq 21600,00$ \\
\hline 10 & 387379301,999975 & 387382382 & - & - & - & - & - & $\geq 21600,00$ \\
\hline \multicolumn{9}{|c|}{ Modelo $\mathcal{Q}_{3}^{G}$} \\
\hline \multirow[t]{2}{*}{ \# Instância } & \multicolumn{5}{|c|}{ Dados das soluções } & \multicolumn{3}{|c|}{ Medidas de desempenho } \\
\hline & Lim. Inf. & Lim. Sup. & V. Sobras & Qtde. Sobras & Placas Usadas & Iterações MIP & Nós B\&B & Tempo (s) \\
\hline 1 & 590964880,338738 & 590964882 & 94622 & 2 & 1 & 259777 & 113128 & 8,41 \\
\hline 2 & 360588000,666667 & 360588001 & 38400 & 1 & 1 & 1442 & 449 & 0,23 \\
\hline 3 & 759012571,111103 & 759012572 & 96444 & 2 & 1 & 23662 & 5932 & 0,71 \\
\hline 4 & 316397062,324176 & 316397083 & 36023 & 2 & 1 & 7427939 & 1369413 & 140,55 \\
\hline 5 & 186259700,352250 & 186259702 & 39160 & 2 & 1 & 639623 & 105824 & 10,88 \\
\hline 6 & & & - & - & - & - & - & $\geq 21600,00$ \\
\hline 7 & & & - & - & - & - & - & $\geq 21600,00$ \\
\hline 8 & 118951360,147339 & 118951362 & 23584 & 2 & 1 & 255212238 & 41865293 & 4080,08 \\
\hline 9 & 95176245,1444315 & 951762523 & 9880 & 2 & 1 & 175266744 & 21666563 & 2513,87 \\
\hline 10 & 387370060,240973 & 387379302 & - & - & - & - & - & $\geq 21600,00$ \\
\hline
\end{tabular}

Tabela 5.5: Resultados dos experimentos com os modelos $\mathcal{Q}_{0}^{G}, \mathcal{Q}_{1}^{G}, \mathcal{Q}_{2}^{G}$ e $\mathcal{Q}_{3}^{G}$. 


\begin{tabular}{|c|c|c|c|c|c|c|c|c|}
\hline \multicolumn{9}{|c|}{ Modelo $\mathcal{Q}_{0}^{G}$} \\
\hline \multirow[t]{2}{*}{ \# Instância } & \multicolumn{5}{|c|}{ Dados das soluções } & \multicolumn{3}{|c|}{ Medidas de desempenho } \\
\hline & Lim. Inf. & Lim. Sup. & V. Sobras & Qtde. Sobras & Placas Usadas & Iterações MIP & Nós B\&B & Tempo (s) \\
\hline 11 & 100658162,000000 & 100661401 & 23328 & 1 & 1 & 4219 & 1951 & 0,37 \\
\hline 12 & 799741,000000000 & 799741 & 1944 & 1 & 1 & 902 & 174 & 0,19 \\
\hline 13 & 1474620,35714286 & 1474621 & 1512 & 1 & 2 & 275023 & 80396 & 5,12 \\
\hline \multicolumn{9}{|c|}{ Modelo $\mathcal{Q}_{1}^{G}$} \\
\hline \multirow[t]{2}{*}{ \# Instância } & \multicolumn{5}{|c|}{ Dados das soluções } & \multicolumn{3}{|c|}{ Medidas de desempenho } \\
\hline & Lim. Inf. & Lim. Sup. & V. Sobras & Qtde. Sobras & Placas Usadas & Iterações MIP & Nós B\&B & Tempo (s) \\
\hline 11 & 100654920,184524 & 100661401 & 23328 & 1 & 1 & 30621 & 21400 & 1,51 \\
\hline 12 & 799740,166666667 & 799741 & 1944 & 1 & 1 & 6591 & 3301 & 0,49 \\
\hline 13 & 1474619,80951982 & 1474621 & 1512 & 1 & 2 & 2788959 & 1367440 & 77,48 \\
\hline \multicolumn{9}{|c|}{ Modelo $\mathcal{Q}_{2}^{G}$} \\
\hline \multirow[t]{2}{*}{ \# Instância } & \multicolumn{5}{|c|}{ Dados das soluções } & \multicolumn{3}{|c|}{ Medidas de desempenho } \\
\hline & Lim. Inf. & Lim. Sup. & V. Sobras & Qtde. Sobras & Placas Usadas & Iterações MIP & Nós B\&B & Tempo (s) \\
\hline 11 & 100661400,321429 & 100661401 & 23328 & 1 & 1 & 11295387 & 3340773 & 252,84 \\
\hline 12 & 799741,000000000 & 799741 & 1944 & 1 & 1 & 59590 & 16050 & 1,39 \\
\hline 13 & 1468952,00000000 & 1474621 & - & - & - & - & - & $\geq 21600,00$ \\
\hline \multicolumn{9}{|c|}{ Modelo $\mathcal{Q}_{3}^{G}$} \\
\hline \multirow{2}{*}{ \# Instância } & \multicolumn{5}{|c|}{ Dados das soluções } & \multicolumn{3}{|c|}{ Medidas de desempenho } \\
\hline & Lim. Inf. & Lim. Sup. & V. Sobras & Qtde. Sobras & Placas Usadas & Iterações MIP & Nós B\&B & Tempo (s) \\
\hline 11 & 100661392,934083 & 100661401 & 23328 & 1 & 1 & 94947353 & 21366687 & 1820,65 \\
\hline 12 & 799740,933331713 & 799741 & 1944 & 1 & 1 & 570185 & 208469 & 15,72 \\
\hline 13 & 1468952,23809461 & 1474621 & - & - & - & - & - & $\geq 21600,00$ \\
\hline
\end{tabular}

Tabela 5.6: Resultados dos experimentos com os modelos $\mathcal{Q}_{0}^{G}, \mathcal{Q}_{1}^{G}, \mathcal{Q}_{2}^{G}$ e $\mathcal{Q}_{3}^{G}$. 


\section{Capítulo 6}

\section{Conclusões}

Neste trabalho estudamos duas variações do problema de empacotar retângulos idênticos (a menos de rotações de 90 graus) em um poliedro. Um consiste em encontrar a maior quantidade de itens retangulares idênticos que podem ser empacotados em um poliedro. $\mathrm{O}$ outro consiste em encontrar o poliedro de um determinado tipo com menor área para empacotar uma quantidade fixa de itens retangulares idênticos. Apresentamos modelos de programação linear inteira mista para esses problemas e desenvolvemos restrições de eliminação de simetrias que efetivamente melhoram a performance de resolução dos modelos pelo CPLEX (e, consequentemente, outros métodos baseados em branch-\&-bound). É importante mencionar que este trabalho foi aceito para publicação na revista Optimization Letters sob o título Symmetry-breaking constraints for packing identical rectangles within polyhedra.

Abordamos também dois problemas de corte de itens retangulares a partir de objetos retangulares, com sobras aproveitáveis. O primeiro, chamado de problema de corte em dois estágios com sobras aproveitáveis, consiste em encontrar padrões de corte do tipo dois estágios de forma a maximizar o valor das sobras aproveitáveis dentre os padrões que minimizam o custo dos objetos. $\mathrm{O}$ segundo, chamado de problema de corte com sobras aproveitáveis, consiste em encontrar padrões de corte (sem restrições) de forma a maximizar o valor das sobras aproveitáveis dentre os padrões que minimizam o custo dos objetos. Desenvolvemos modelos de programação em dois níveis e os reformulamos como modelos de programação inteira mista. A fim de conseguir resolver instâncias maiores do problema de corte, desenvolvemos restrições de eliminação de soluções equivalentes e de soluções simétricas. Desenvolvemos também uma estratégia em duas fases, que consiste em resolver inicialmente uma versão do problema de corte (sem considerar as sobras aproveitáveis) e, em seguida, a partir desta solução, acrescentar uma restrição ao modelo do problema de corte com sobras aproveitáveis, que é então resolvido.

Os modelos para os dois problemas de corte com sobras aproveitáveis apresentados neste trabalho são propostas novas. Até onde pudemos observar, não existem trabalhos publicados propondo modelos de programação matemática para problemas de corte em duas dimensões com sobras aproveitáveis. Os modelos apresentados para o problema de corte com sobras aproveitáveis foram construídos usando uma mesma estratégia. Ela consiste em modelar o problema de corte com sobras aproveitáveis como um problema de otimização em dois níveis e depois reformulá-lo em um MIP. Neste problema de otimização em dois níveis, o problema do nível inferior consiste em minimizar o custo dos objetos e o problema do nível superior consiste em maximizar o valor das sobras aproveitáveis. A reformulação baseia-se no fato de que a função objetivo do problema do nível inferior está desacoplada das variáveis do nível superior, ou seja, as variáveis do problema do nível superior não influenciam o valor da função objetivo do problema do nível inferior. A função objetivo do modelo reformulado consiste na função objetivo do problema do nível superior normalizada, de forma a assumir valores no intervalo $[0,1)$, subtraída da função objetivo do problema 
do nível inferior. A parte inteira da nova função objetivo está associada ao valor da função objetivo do problema do nível inferior e a parte fracionária, desta nova função, está relacionada com a função objetivo do nível superior. Dessa forma fica claro que a função objetivo do problema do nível superior (parte fracionária) é utilizada para desempatar entre soluções do problema do nível inferior (parte inteira). As restrições da reformulação são compostas pelas restrições dos problemas do nível superior e inferior.

Finalmente, considerando a questão da quantidade de sobras aproveitáveis, modelamos o problema no qual deseja-se obter, dentre as soluções que minimizam o custo dos objetos, aquelas que maximizam o custo das sobras e, dentre estas últimas, uma que minimize a quantidade de sobras aproveitáveis. Desenvolvemos modelos MIPs para este problema, considerando os diferentes tipos de sobras aproveitáveis definidos no Capítulo 4, a partir dos modelos $\mathcal{S}_{0}^{G}, \mathcal{S}_{1}^{G}, \mathcal{S}_{2}^{G}$ e $\mathcal{S}_{3}^{G}$, considerando que esse novo problema pode ser modelado como um problema de otimização em três níveis, de forma similar a que fizemos com o problema de corte com sobras em dois níveis.

Considerando a dificuldade em resolver os modelos desenvolvidos e o fato de termos conseguido apenas resolver instâncias de tamanho pequeno, acreditamos que uma das possibilidades de pesquisa futura seja desenvolver métodos heurísticos ou híbridos para resolver esses modelos. 


\section{Referências Bibliográficas}

[Abuabara08] A. Abuabara and R. Morabito. "Cutting optimization of structural tubes to build agricultural light aircrafts". Annals of Operations Research, Vol. 169, No. 1, pp. 149-165, sep 2008. 5

[Alvarez-Valdes02] R. Alvarez-Valdes, A. Parajon, and J. M. Tamarit. "A computational study of LP-based heuristic algorithms for two-dimensional guillotine cutting stock problems". OR Spectrum, Vol. 24, No. 2, pp. 179-192, May 2002. 5

[Arenales95] M. N. Arenales and R. Morabito. "An AND/OR-graph approach to the solution of two-dimensional non-guillotine cutting problems". European Journal of Operational Research, Vol. 84, No. 3, pp. 599-617, 1995. 4

[Babu99] A. R. Babu and N. R. Babu. "Effective nesting of rectangular parts in multiple rectangular sheets using genetic and heuristic algorithms". International Journal of Production Research, Vol. 37, No. 7, pp. 1625-1643, May 1999. 5

[Balas98] E. Balas. "Disjunctive programming: Properties of the convex hull of feasible points". Discrete Applied Mathematics, Vol. 89, No. 1, pp. 1-44, 1998. 9

[Bard98] J. Bard. Practical Bilevel Optimization: Applications and Algorithms. Kluwer Academic Publishers, 1998. 2

[Beasley85a] J. E. Beasley. "Algorithms for unconstrained two-dimensional guillotine cutting". Journal of the Operational Research Society, Vol. 36, No. 4, pp. 297306, 1985. 2, 4, 25

[Beasley85b] J. E. Beasley. "An exact two-dimensional non-guillotine cutting tree search procedure”. Operations Research, Vol. 33, No. 1, pp. 49-64, Jan. 1985. 4, 7

[Birgin05a] E. G. Birgin, J. M. Martinez, W. F. Mascarenhas, and D. P. Ronconi. "Method of sentinels for packing items within arbitrary convex regions". Journal of the Operational Research Society, Vol. 57, No. 6, pp. 735-746, Sep. 2005. 3, 7

[Birgin05b] E. G. Birgin, R. Morabito, and F. H. Nishihara. "A note on an L-approach for solving the manufacturers pallet loading problem". Journal of the Operational Research Society, Vol. 56, No. 12, pp. 1448-1451, Dec. 2005. 3, 7

[Birgin06] E. G. Birgin, J. M. Martinez, F. H. Nishihara, and D. P. Ronconi. "Orthogonal packing of rectangular items within arbitrary convex regions by nonlinear optimization". Computers \& Operations Research, Vol. 33, No. 12, pp. 35353548, Dec. 2006. 3, 7 
[Birgin09] E. G. Birgin and R. D. Lobato. "Orthogonal packing of rectangles within isotropic convex regions". Computers \& Industrial Engineering, pp. 1-16, 2009. 3, 7

[Birgin10a] E. G. Birgin, R. D. Lobato, and R. Morabito. "An effective recursive partitioning approach for the packing of identical rectangles in a rectangle". Journal of the Operational Research Society, Vol. 61, No. 2, pp. 306-320, 2010. 3, 7

[Birgin10b] E. G. Birgin and W. F. Mascarenhas. "Using sentinels to detect intersections of convex and nonconvex polygons". Computational \& Applied Mathematics, Vol. 29, No. 2, pp. 247-267, 2010. 3, 7

[Birgin11] E. G. Birgin, R. D. Lobato, and R. Morabito. "Generating unconstrained two-dimensional non-guillotine cutting patterns by a Recursive Partitioning Algorithm". Journal of the Operational Research Society, Vol. 63, No. 2, pp. 183-200, Aug. 2011. 7

[Bischoff95] E. Bischoff and G. Wäscher. "Cutting and packing". European Journal of Operational Research, Vol. 84, No. 3, pp. 503-505, Aug. 1995. 2

[Cassioli11] A. Cassioli and M. Locatelli. "A heuristic approach for packing identical rectangles in convex regions". Computers \& Operations Research, Vol. 38, No. 9, pp. 1342-1350, Sep. 2011. 3, 7

[Cherri09a] A. C. Cherri. Algumas extensões do problema de corte de estoque com sobras de material aproveitáveis. Tese de Doutorado, Universidade de São Paulo, Feb. 2009. 1, 5

[Cherri09b] A. C. Cherri, M. N. Arenales, and H. H. Yanasse. "The one-dimensional cutting stock problem with usable leftover - A heuristic approach". European Journal of Operational Research, Vol. 196, No. 3, pp. 897-908, Aug. 2009. 5

[Christofides77] N. Christofides and C. Whitlock. "An Algorithm for Two-Dimensional Cutting Problems". Operations Research, Vol. 25, No. 1, pp. 30-44, Jan. 1977. 4

[Chu99] C. Chu and J. Antonio. "Approximation Algorithms to Solve Real-Life Multicriteria Cutting Stock Problems". Operations Research, Vol. 47, No. 4, pp. 495-508, July 1999. 5

[Cui11] Y. Cui, T. Gu, and W. Hu. "Recursive algorithms for the optimum cutting of equal rectangles". International Journal of Computers and Applications, Vol. 33, No. 2, 2011. 4

[Cui12] Y. Cui and B. Huang. "Reducing the number of cuts in generating three-staged cutting patterns". European Journal of Operational Research, Vol. 218, No. 2, pp. 358-365, Apr. 2012. 5

[Cung00] V.-D. Cung, M. Hifi, and B. Cun. "Constrained two-dimensional cutting stock problems a best-first branch-and-bound algorithm". International Transactions in Operational Research, Vol. 7, No. 3, pp. 185-210, May 2000. 4

[Dempe02] S. Dempe. Foundations of bilevel programming. Springer, 2002. 2, 28 
[Dowsland92] K. A. Dowsland and W. B. Dowsland. "Packing problems". European Journal of Operational Research, Vol. 56, No. 1, pp. 2-14, Jan. 1992. 2

[Dyckhoff90] H. Dyckhoff. "A typology of cutting and packing problems". European Journal of Operational Research, Vol. 44, No. 2, pp. 145-159, Jan. 1990. 3, 4

[Dyckhoff97] H. Dyckhoff, G. Scheithauer, and J. Terno. "Cutting and Packing". In: S. Martello, M. Dell'Amico, and F. Maffioli, Eds., Annotated Bibliographies in Combinatorial Optimization, Chap. 22, pp. 393-413, John Wiley and Sons, 1997. 2

[Finke92] U. Finke and H. Dyckhoff. Cutting and Packing in Production and Distribution: A Typology and Bibliography. Physica-Verlag, Heidelberg, 1992. 2

[Fischer81] M. L. Fischer. "The Lagrangian Relaxation Method for Solving Integer Programming Problems". Management Science, Vol. 27, No. 1, pp. 1-18, 1981. 4

[Gilmore63] P. C. Gilmore and R. E. Gomory. "A Linear Programming Approach to the Cutting Stock Problem-Part II". Operations Research, Vol. 11, No. 6, pp. 863-888, Nov. 1963. 5

[Gilmore65] P. C. Gilmore and R. E. Gomory. "Multistage Cutting Stock Problems of Two and More Dimensions". Operations Research, Vol. 13, No. 1, pp. 94-120, Jan. 1965. 2, 4, 5, 25

[Gradisar05] M. Gradisar and P. Trkman. "A combined approach to the solution to the general one-dimensional cutting stock problem". Computers \& Operations Research, Vol. 32, No. 7, pp. 1793-1807, July 2005. 5

[Harjunkoski97] I. Harjunkoski, R. Skrifvars, and T. Westerlund. "Different strategies for solving bilinear integer non-linear programming problems with convex transformations". Computers \& Chemical Engineering, Vol. 21, pp. S487-S492, 1997. 64

[Herz72] J. C. Herz. "Recursive Computational Procedure for Two-dimensional Stock Cutting". IBM Journal of Research and Development, Vol. 16, No. 5, pp. 462469, Sep. 1972. 4

[Hifi01] M. Hifi and C. Roucairol. "Approximate and Exact Algorithms for Constrained ( Un ) Weighted Two-dimensional Two-staged Cutting". Journal of Combinatorial Optimization, Vol. 5, No. 4, pp. 465-494, 2001. 36

[Hifi97] M. Hifi. “An improvement of Viswanathan and Bagchi's exact algorithm for constrained two-dimensional cutting stock". Computers \& operations research, Vol. 24, No. 8, pp. 727-736, 1997. 4

[Kallrath08] J. Kallrath. "Cutting circles and polygons from area-minimizing rectangles". Journal of Global Optimization, Vol. 43, No. 2-3, pp. 299-328, Jan. 2008. 7

[Korf08] R. E. Korf, M. D. Moffitt, and M. E. Pollack. "Optimal rectangle packing”. Annals of Operations Research, Nov. 2008. 3 
[Land60] A. H. Land and A. G. Doig. "An Automatic Method of Solving Discrete Programming Problems”. Econometrica, Vol. 28, No. 3, p. 497, July 1960. 1

[Li96] S. Li. "Multi-job Cutting Stock Problem with Due Dates and Release Dates". Journal of the Operational Research Society, Vol. 47, No. 4, pp. 490-510, Apr. 1996. 5

[Lins03] L. Lins, S. Lins, and R. Morabito. “An L-approach for packing (1, w)rectangles into rectangular and L-shaped pieces". Journal of the Operational Research Society, Vol. 54, No. 7, pp. 777-789, 2003. 3

[Lodi02] A. Lodi, S. Martello, and M. Monaci. "Two-dimensional packing problems: A survey". European Journal of Operational Research, Vol. 141, No. 2, pp. 241-252, Sep. 2002. 2, 3

[Lodi03] A. Lodi and M. Monaci. "Integer linear programming models for 2-staged two-dimensional Knapsack problems". Mathematical Programming, Vol. 94, No. 2-3, pp. 257-278, 2003. 2, 6, 7, 26, 34, 35

[Morabito00] R. Morabito and M. N. Arenales. "Optimizing the cutting of stock plates in a furniture company". International Journal of Production Research, Vol. 38, No. 12, pp. 2725-2742, 2000. 1

[Morabito08] R. Morabito and V. Pureza. "A heuristic approach based on dynamic programming and and/or-graph search for the constrained two-dimensional guillotine cutting problem". Annals of Operations Research, Vol. 179, No. 1, pp. 297-315, Nov. 2008. 4

[Morabito12] R. Morabito and H. H. Yanasse. "Modelos lineares e não-lineares inteiros para problemas da mochila bidimensional restrita a dois estágios". Produção, 2012. To appear. 26

[Morabito89] R. Morabito. Corte de Estoque Bidimensional. Tese de Doutorado, Universidade de São Paulo, 1989. 4

[Morabito92] R. Morabito, M. Arenales, and V. Arcaro. "An and-or-graph approach for two-dimensional cutting problems". European Journal of Operational Research, Vol. 58, No. 2, pp. 263-271, Apr. 1992. 4

[Morabito94] R. Morabito and M. Arenalest. "An AND/OR-graph Approach to the Container Loading Problem". International Transactions in Operational Research, Vol. 1, No. 1, pp. 59-73, Jan. 1994. 4

[Morabito96] R. Morabito and M. N. Arenales. "Staged and constrained two-dimensional guillotine cutting problems: An AND/OR-graph approach". European Journal of Operational Research, Vol. 94, No. 3, pp. 548-560, Nov. 1996. 2, 4, 25

[Morabito98] R. Morabito and S. Morales. "A simple and effective recursive procedure for the manufacturer's pallet loading problem". Journal of the Operational Research Society, Vol. 49, No. 8, pp. 819-828, Aug. 1998. 1, 3 
[Mornar97] V. Mornar and B. Khoshnevis. "A cutting stock procedure for printed circuit board production". Computers \& Industrial Engineering, Vol. 32, No. 1, pp. 57-66, Jan. 1997. 5

[Ostrowski10] J. Ostrowski, J. Linderoth, F. Rossi, and S. Smriglio. "Solving Steiner Triple Covering Problems". Optima, pp. 1-7, July 2010. 11

[Poldi09] K. C. Poldi and M. N. Arenales. "Heuristics for the one-dimensional cutting stock problem with limited multiple stock lengths". Computers \& Operations Research, Vol. 36, No. 6, pp. 2074-2081, June 2009. 5

[Raman94] R. Raman and I. Grossmann. "Modeling and computational techniques for logic based integer programming". Computers and Chemical Engineering, Vol. 18, No. 7, pp. 563-578, 1994. 9

[Riehme96] J. Riehme, G. Scheithauer, and J. Terno. “The solution of two-stage guillotine cutting stock problems having extremely varying order demands". European Journal of Operational Research, Vol. 91, No. 3, pp. 543-552, June 1996. 5

[Roodman86] G. M. Roodman. "Near-optimal solutions to one-dimensional cutting stock problems". Computers \& Operations Research, Vol. 13, No. 6, pp. 713-719, Jan. 1986. 5

[Sawaya05] N. W. Sawaya and I. E. Grossmann. "A cutting plane method for solving linear generalized disjunctive programming problems". Computers \& Chemical Engineering, Vol. 29, No. 9, pp. 1891-1913, Aug. 2005. 8

[Scheithauer91] G. Scheithauer. "A note on handling residual lengths". Optimization, Vol. 22, No. 3, pp. 461-466, 1991. 5

[Sinuany-Stern94] Z. Sinuany-Stern and I. Weiner. "The One Dimensional Cutting Stock Problem Using Two Objectives". The Journal of the Operational Research Society, Vol. 45, No. 2, pp. 231-236, 1994. 5

[Stoyan98] Y. G. Stoyan and G. N. Yaskov. "Mathematical Model and Solution Method of Optimization Problem of Placement of Rectangles and Circles Taking into Account Special Constraints". International Transactions in Operational Research, Vol. 5, No. 1, pp. 45-57, Jan. 1998. 3

[Sweeney92] P. E. Sweeney and E. R. Paternoster. "Cutting and Packing Problems: A Categorized, Application-Orientated Research Bibliography". The Journal of the Operational Research Society, Vol. 43, No. 7, pp. 691-706, 1992. 2

[Vicente94] L. N. Vicente and P. H. Calamai. "Bilevel and multilevel programming: A bibliography review". Journal of Global Optimization, Vol. 5, No. 3, pp. 291306, Oct. 1994. 28

[Viswanathan93] K. V. Viswanathan and A. Bagchi. "Best-First Search Methods for Constrained Two-Dimensional Cutting Stock Problems". Operations Research, Vol. 41, No. 4, pp. 768-776, July 1993. 4

[Wang83] P. Y. Wang. "Two Algorithms for Constrained Two-Dimensional Cutting Stock Problems". Operations Research, Vol. 31, No. 3, pp. 573-586, May 1983. 4 
[Wäscher07] G. Wäscher, H. Haußner, and H. Schumann. "An improved typology of cutting and packing problems". European Journal of Operational, Vol. 183, No. 3, pp. 1109-1130, 2007. 1, 3, 4, 5

[Yanasse06] H. H. Yanasse and R. Morabito. "Linear models for 1-group two-dimensional guillotine cutting problems". International Journal of Production Research, Vol. 44, No. 17, pp. 3471-3491, Sep. 2006. 64 